\title{
QUALITY OF LIFE AMONG DIALYSIS PATIENTS IN UNITED ARAB EMIRATES
}

\author{
By \\ Abdelbasit Mohamad Abdelhadi Ayoub
}
A thesis submitted to the Victoria University of Wellington in the fulfilment of the requirements for the degree of Doctor of Philosophy in Nursing

Victoria University of Wellington 2012 


\begin{abstract}
Background: Quality of Life (QOL) has emerged as an important parameter for evaluating the quality of health care for patients with renal failure. The QOL of dialysis patients in the United Arab Emirates (UAE) has not previously been studied. This research examined the cultural relevancy of two QOL tools and compared the QOL between dialysis patients and a sample from the community in UAE.
\end{abstract}

Method: A descriptive comparative survey design was used to study the QOL of 161 dialysis patients and 350 participants from the community. Dialysis patients completed the SF-36 and the QOL Index dialysis version tool. Participants from the community completed the SF-36 and QOL Index generic version tool. Both samples also completed three open-ended questions about the cultural relevancy of the tools. Analysis using descriptive and inferential statistics was done within and between groups to establish similarities and differences in QOL scores. Open-ended questions were analysed thematically.

Results: The overall QOL for dialysis patients was rated lower when self-assessed using the SF-36 compared with the community sample (58.92 vs. $75.02, p=<0.0001$ ). Furthermore, the overall QOL for dialysis patients was rated almost the same when self-assessed using the QOL Index compared with the community sample (77.2 vs. 78.6, $p=0.421$ ). Moreover, the total scores of both tools were higher in the community sample (75.02 vs. 78.6). The comparison between the statistically significant variables for both samples revealed contradictory results with the two tools. The majority of participants considered both tools culturally relevant in general. There were more questions that were not answered by participants in the QOL Index by both samples compared with the SF-36.

Conclusion: In the UAE the SF 36 and the QOL Index capture different aspects of quality of life. Clinicians and researchers need to be aware of these differences when using these tools. An effective way of establishing the cultural relevancy of QOL tools involves comparing the QOL of a group with a health condition with a sample from the community, asking the participants about their views of the cultural relevancy of the tools, examining missing data, and asking participants open-ended questions about what they value in life. 
Key words: Quality of life, Dialysis, Islamic religion, United Arab Emirates, Cultural relevancy. 


\section{Acknowledgment}

Firstly, my greatest debt of gratitude goes to my wife Rudaina, for all of her patience and the continuous encouragement she has shown me whilst undertaking this study. Without her support and understanding I would not have got through this long journey. The love and support she gave me all my life is why I have been able to achieve the goals I worked so hard to get to.

I would like to thank my children Usama, Annas and Ayham for their appreciation and for their understanding towards the fact that their father needed to be away for his studies and research. Without their support I would not have got through to the end.

Also my appreciation goes out to all the participants and the renal unit staff at the Sheikh khalifa Medical City in the UAE for their willingness to help and support throughout my study. In particular, I would like to give a big thanks to Dr. Kamal Hijjazi and Dr. Abdulkareem Saleh for encouraging my research and facilitating the data collection.

To the Graduate School of Nursing, Midwifery and Health at the Victoria University of Wellington I send my kind gratitude for their support and guidance, especially for their generous support while I was studying over the last few years. A very special thank you is well deserved by my primary supervisor Senior Lecturer, Dr Kathy Nelson and my second supervisor Associate Professor, Pamela Wood for their encouragement, guidance and the opportunity they have given me to develop and grow through this journey. A big thank you also to the administrative staff at the Graduate School of Nursing, Midwifery and Health: Abbey McDonald, Pam Green and Chris Fox for their help and support. Special thanks to the Faculty of Humanities and Social Sciences for the some research funding and to the statisticians (Dr Noktuhaba Sibanda and Dr Dalice Sim) at Victoria University of Wellington for their advice.

Lastly, special thanks must go to Professor Ken Walsh who inspired me to start the $\mathrm{PhD}$ project. Also, I would like to thank all of my friends and colleagues who have supported me through this journey. 


\section{Table of Contents}

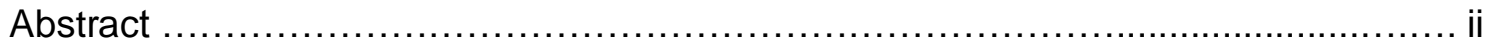

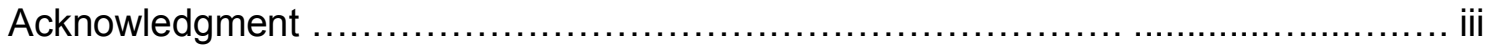

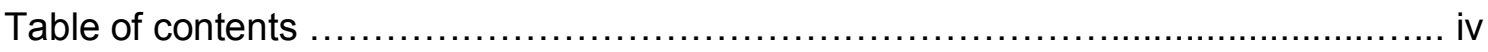

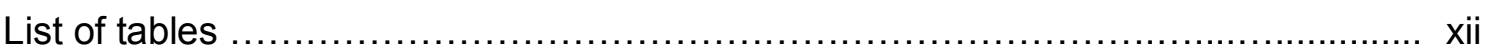

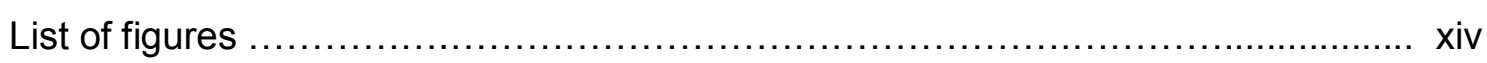

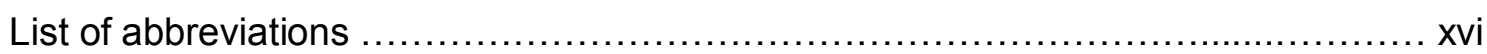

Chapter 1 Introduction and overview .........................................................

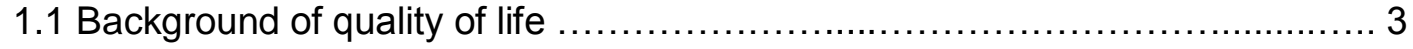

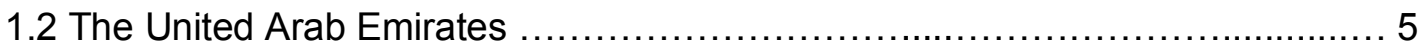

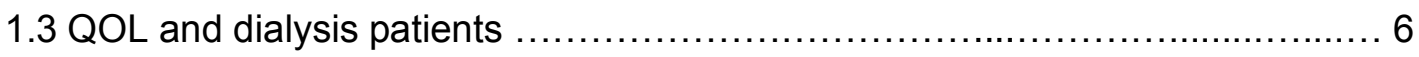

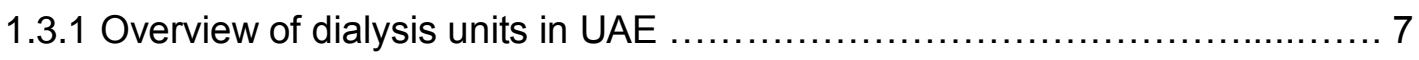

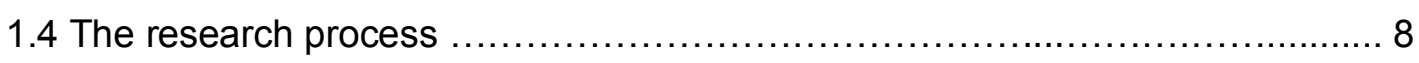

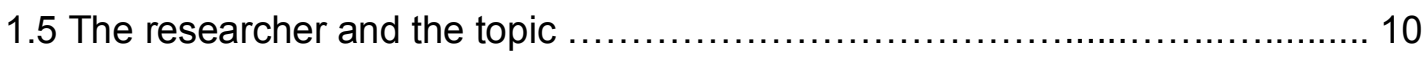

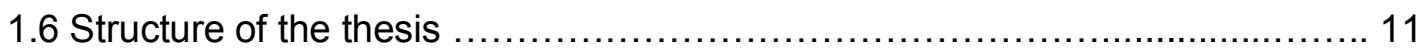

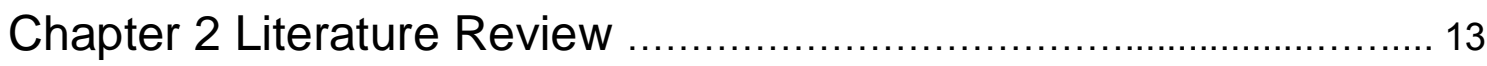

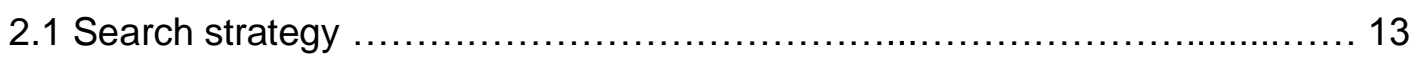

2.2 The notions or interest of studying QOL concept .............................. 14

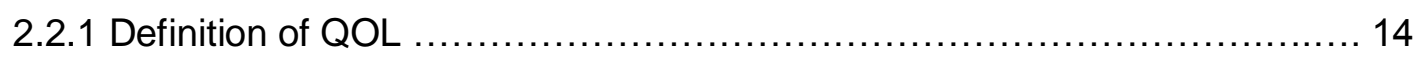

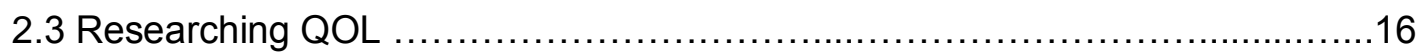

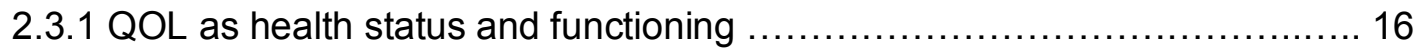

2.3.2 Objective and subjective dimensions of QOL ............................ 17

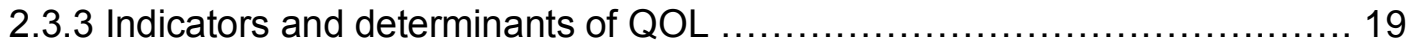

2.3.4 Negative and positive components ..................................... 21

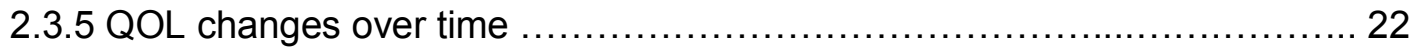

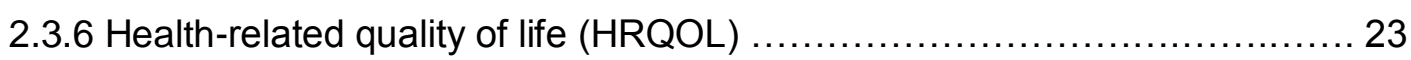

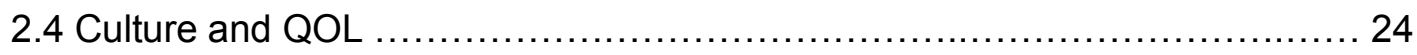

2.5 QOL in Dialysis Patients ............................................... 26

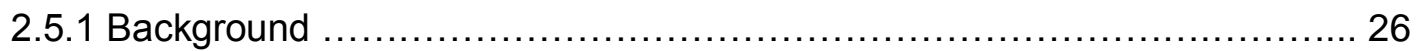

2.6 Socio-demographic factors clinical variables contributing to QOL in dialysis

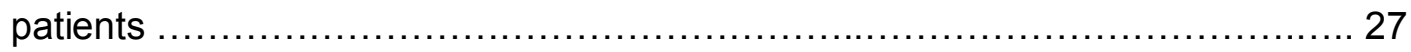

2.6.1 Socio-demographic factors contributing to $\mathrm{QOL}$ in dialysis patients ........ 27

2.6.2 Clinical variables contributing to $\mathrm{QOL}$ in dialysis patient ..................... 33 
2.7 Literature gaps 38

2.8 Conclusion

Chapter 3 Quality of life tools and the conceptual framework 43

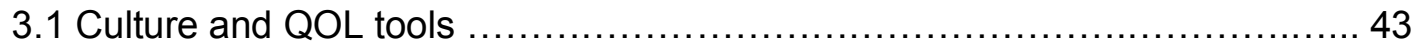

3.1.1 Why assessing cultural relevancy of $\mathrm{QOL}$ tools? ......................... 44

3.1.2 Methods of translations and cross-cultural adaptation of QOL tools .......... 45

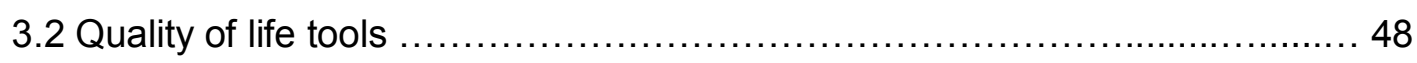

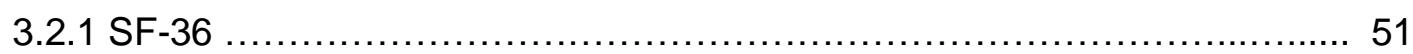

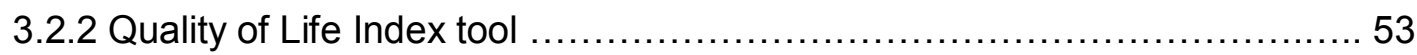

3.2.3 Points related to critiquing QOL tools ................................... 57

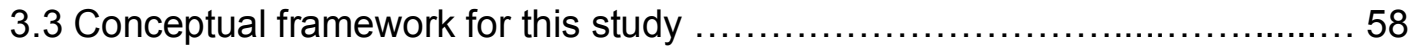

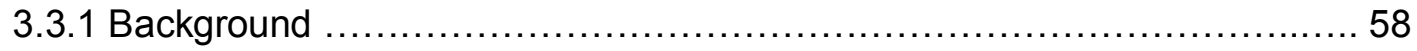

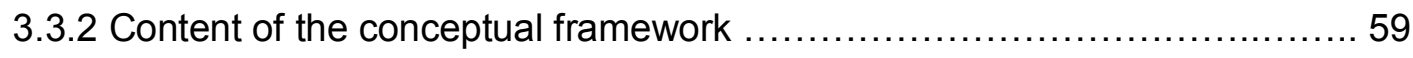

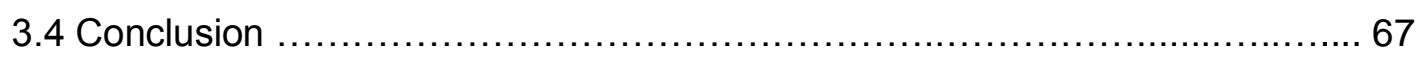

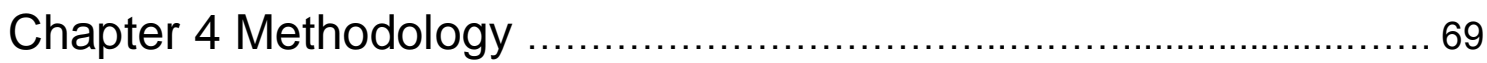

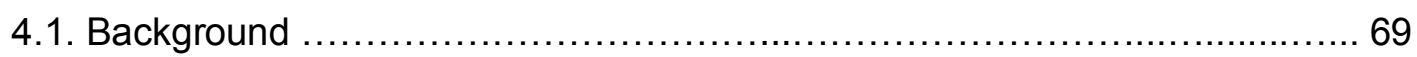

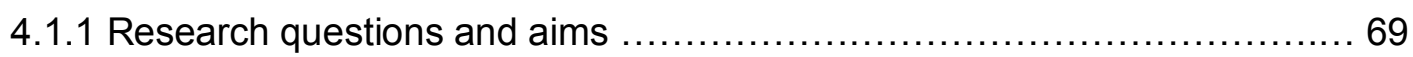

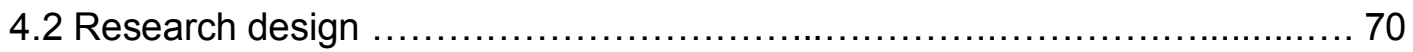

4.2.1 Advantages and disadvantages of descriptive survey design ............... 72

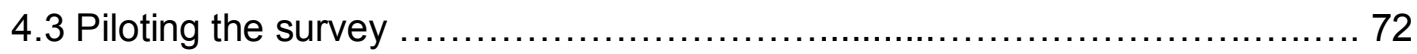

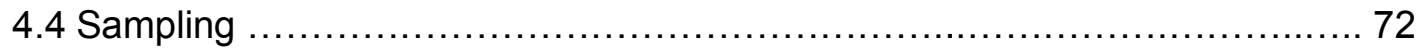

4.4.1 Dialysis sample and recruitment of participants ....................... 72

4.4.2 Community sample and recruitment of participants ....................... 73

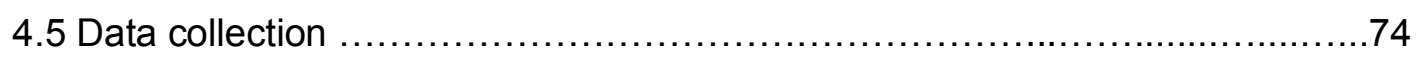

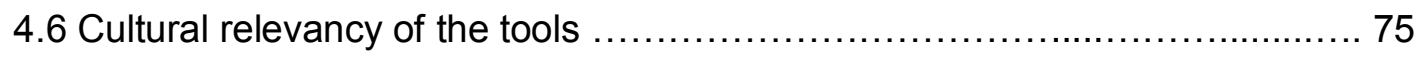

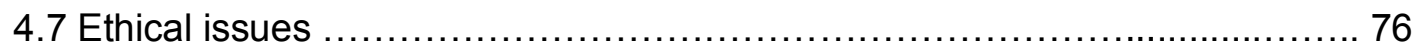

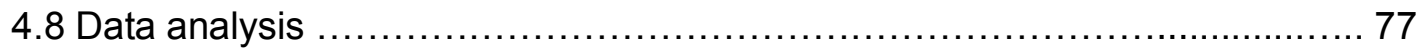

4.8.1 Establishing and preparing the data base (data entry) $\ldots \ldots \ldots \ldots \ldots \ldots \ldots .77$

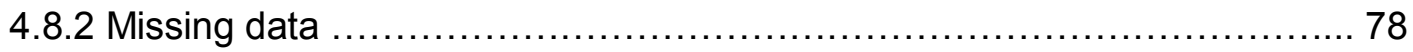

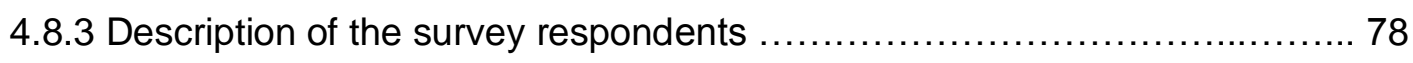

4.8.4 Blood results of the dialysis sample ................................. 79

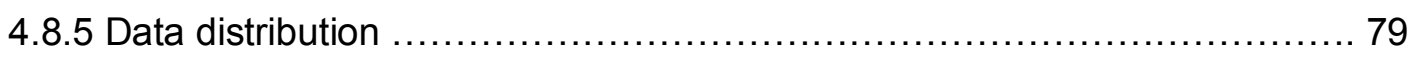

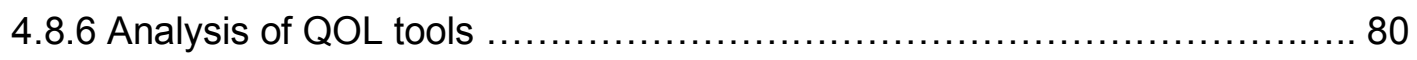


4.8.7 Qualitative data 80

4.8.8 Correlations and differences of socio-demographic and life factors with QOL total scores

4.8.9 Demographic differences and similarities between the dialysis and the

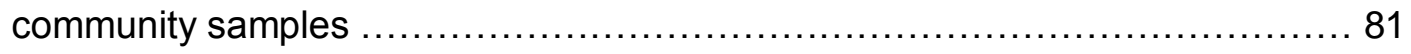

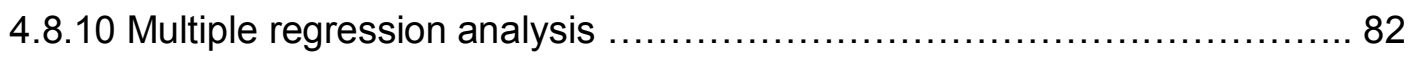

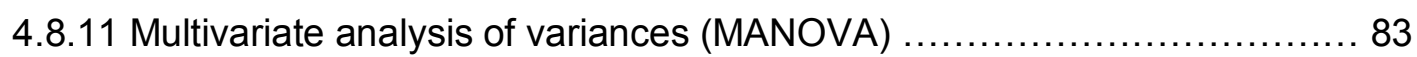

Chapter 5 Findings related to the samples and the cultural relevancy 87

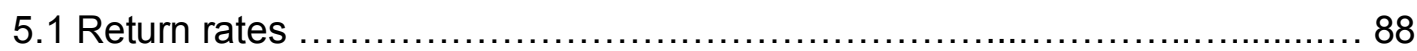

5.2 Demographic and clinical data findings of both samples ....................... 88

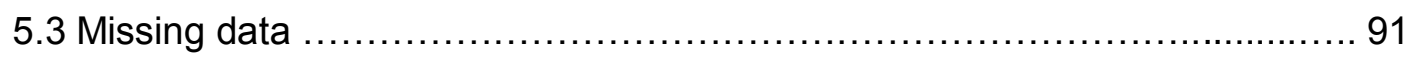

5.3.1 Cultural relevancy in relation to missing data from QOL Index tool subscales 93

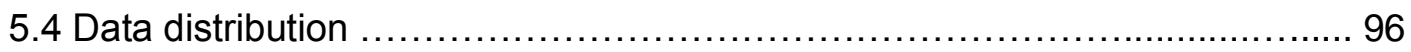

5.5 Cultural relevancy of tools ................................................... 100

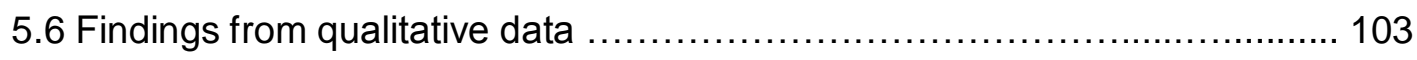

5.6.1 Comparison of the responses to open-ended questions for both samples 105

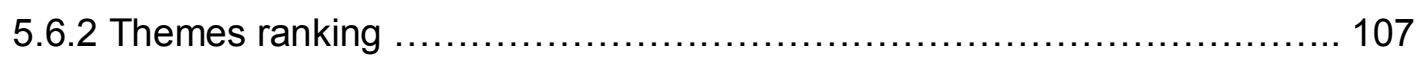

5.6.3 Themes representations in the QOL tools ............................... 108

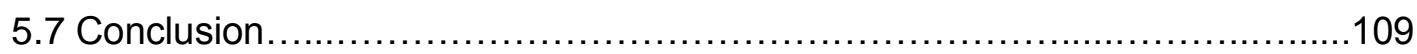

Chapter 6 Discussion on sample characteristics and cultural relevancy

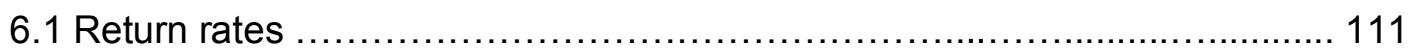

6.2 Demographic and clinical data of both samples ...............................112

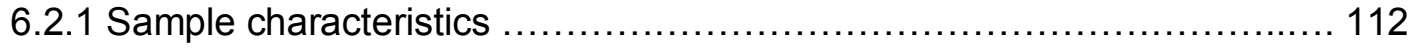

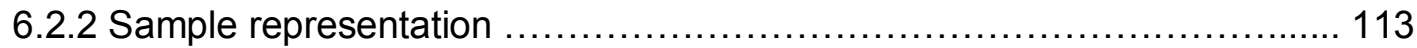

6.2.3 The demographic similarities and differences of the samples ............. 114

6.3 Cultural relevancy of the tools ............................................. 115

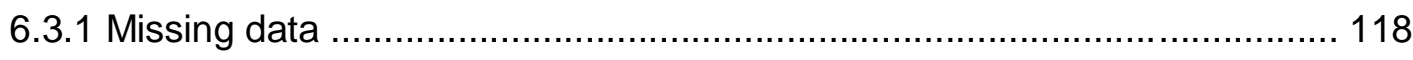


6.3.2 Comparison of the characteristics of the respondents who did not answer the questions about satisfaction with and importance of sex and spouse for both groups 120

6.3.3 Respondents views on questions added or deleted from QOL tools 124

6.4 The usage of the QOL tools in clinical settings .............................. 129

6.5 Respondents' views on QOL ................................................ 131

6.5.1 Ranking of the identified themes .......................................... 134

6.5.2 Themes identified and the level of representation in the QOL tools ......... 134

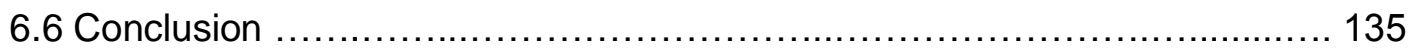

Chapter 7 Findings related to Quality of life ................................ 137

7.1 Findings from QOL tools and their subscales analyses ..................... 137

7.1.1 Blood results of the dialysis sample ................................... 139

7.2 The relationship between the SF-36 total scores and the independent variables in both samples 140

7.3 The relationship between the QOL Index total scores and the independent variables in both samples ............................................................ 143

7.4 Comparison of the significant values of the two tools ......................... 146

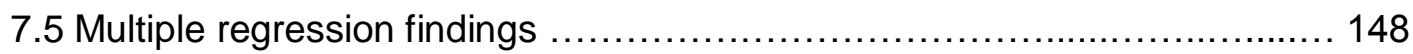

7.5.1 Multiple regression results dialysis sample using SF-36 ................... 148

7.5.2 Multiple regression analysis for the dialysis sample using QOL Index .......151

7.5.3 Multiple regression results for the community sample using SF-36 ........ 153

7.5.4 Multiple regression results for the community sample using QOL Index

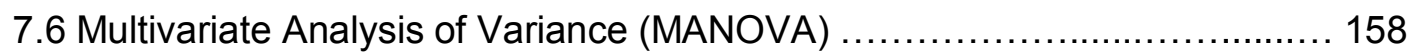

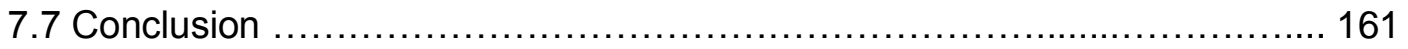

Chapter 8 Discussion of the findings from the tools ....................... 163

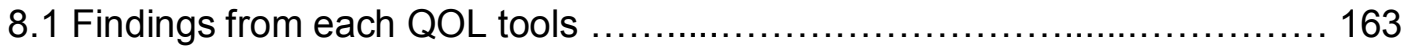

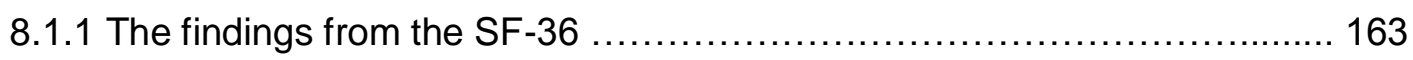

8.1.2 Comparison between the SF-36 subscales of the community samples with the normative values from international studies ................................. 166

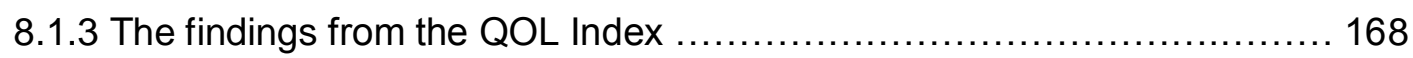

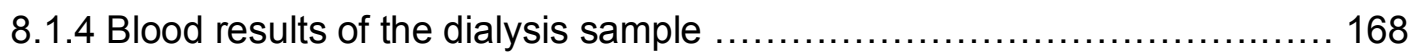


8.2 The relationship between the socio-demographic variables and the total scores of both tools in both samples 170

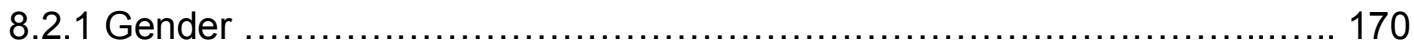

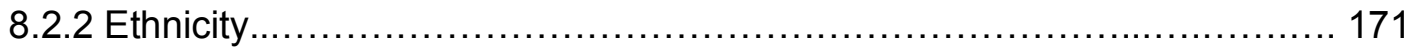

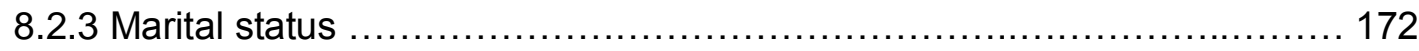

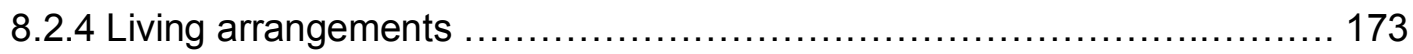

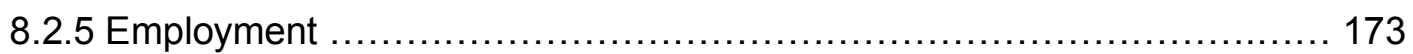

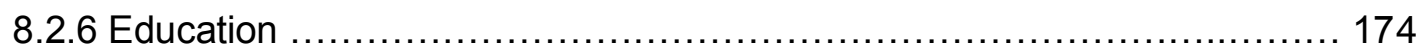

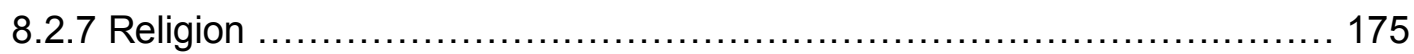

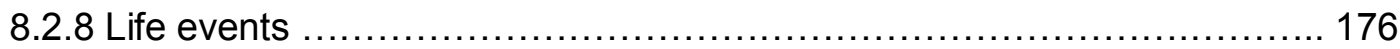

8.2.9 The length of time lived in the UAE and the last time respondents travelled

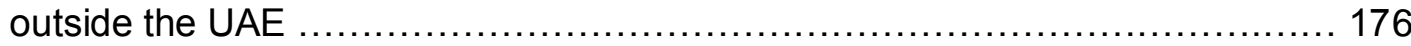

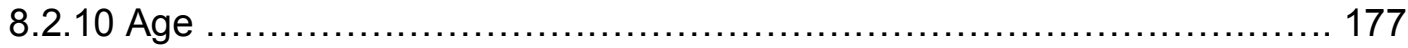

8.3 The relationship between the clinical variables and the total scores of both tools in the dialysis sample .................................................. 178

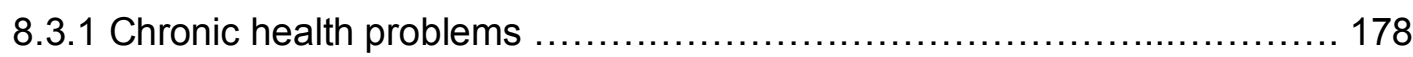

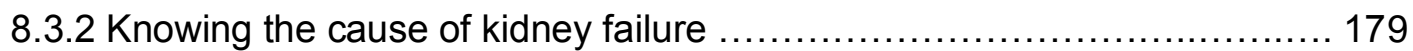

8.3.3 Length of time on dialysis ................................................ 179

8.3.4 Correlation between the total scores of both tools and blood results ....... 180

8.4 Comparison of the significant values of the two tools .......................... 182

8.5 Key demographic and clinical determinant of QOL scores ..................... 184

8.6 Key demographic and clinical predictors of QOL scores....................... 187

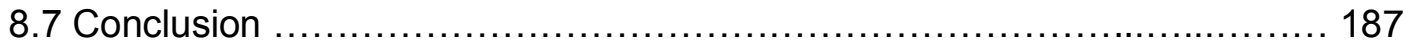

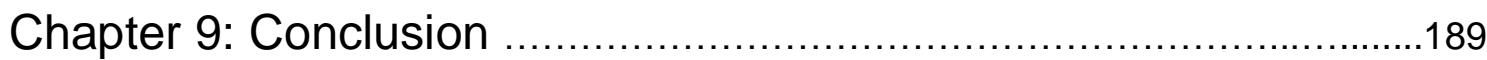

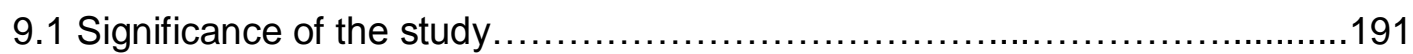

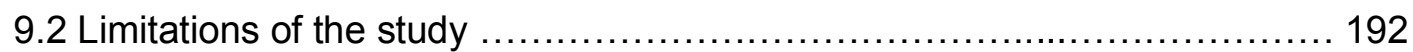

9.3 Implications for practice and nursing care ...................................195

9.4 Implications for research ................................................... 196

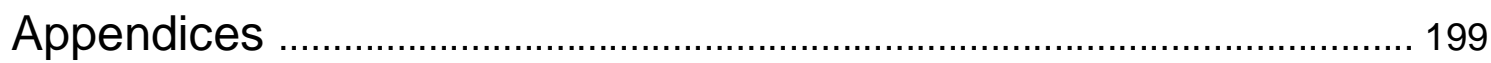

Appendix 1 Ethical approval from Victoria University Human Ethics Committee ....... 199

Appendix 2 Ethical approval from SKMC Ethics Committee ................................... 200

Appendix 3 Invitation letter for dialysis patients ........................................................ 201

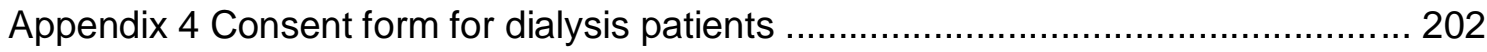


Appendix 5 Survey tools for dialysis patients

Appendix 6 Invitation letter for community sample 223

Appendix 7 Consent form for community sample 225

Appendix 8 Survey tools for community sample 230

Appendix 9 Table A1 missing data for the SF-36

References 249

Bibliography 280 


\section{List of Tables}

Table 1.1 Summary of dialysis patient data for the dialysis units in UAE ................... 8

Table 2.1 Examples of definitions of QOL ................................................................... 16

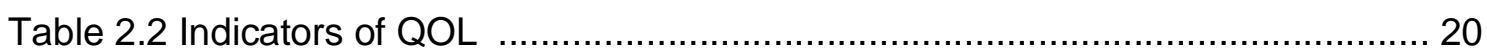

Table 3.1 Examples of general tools used to measure quality of life .......................... 49

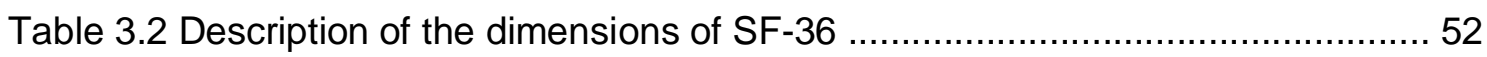

Table 3.3 Assessment of the SF-36 against the selection criteria ….......................... 54

Table 3.4 Assessment of the QOL Index against the selection criteria ....................... 56

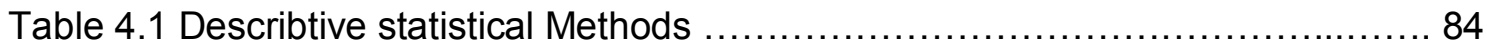

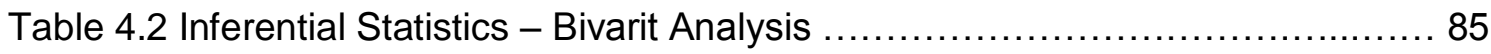

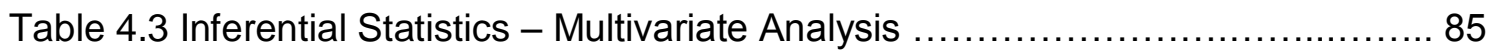

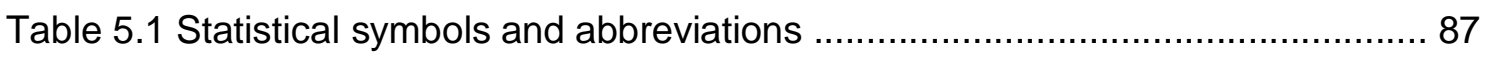

Table 5.2 Comparison of the demographics of the dialysis and community samples

Table 5.3 Comparison of continuous socio-demographic factors for both samples .....90

Table 5.4 Characteristics of respondents who missed questions on the SF-36 ........92

Table 5.5 Characteristics of respondents who missed questions on the QOL index ...92

Table 5.6 Questions that had highest missing responses in the QOL Index tool ....... 93

Table 5.7 Missing data in relation to subscales of QOL Index for dialysis sample ...... 94

Table 5.8 Missing data in relation to subscales of QOL Index for community sample

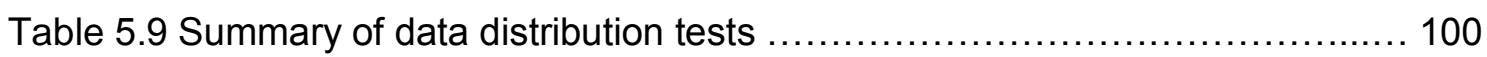

Table 5.10 Comparison between the cultural relevancies of both tools ................ 100

Table 5.11 Demographic characteristics of the respondents who considered QOL tools

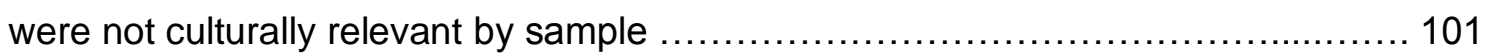

Table 5.12 Dialysis respondents' opinion on the QOL tools ......................... 103

Table 5.13 Missing data of the open-ended questions .............................. 103

Table 5.14 Responses of open-ended questions for both samples ................... 104

Table 5.15 Comparison of themes ranking in both samples ....................... 107

Table 5.16 Representation of the themes in the SF-36 and QOL Index ............... 109

Table 6.1 Comparison of the characteristics of the respondents who did not answer the question about satisfaction with and importance of sex and spouse for both samples

Table 7.1 Comparison between the SF-36 subscales and the total scores of both samples. 
Table 7.2 Comparison between subscales and total scores of QOL Index of both

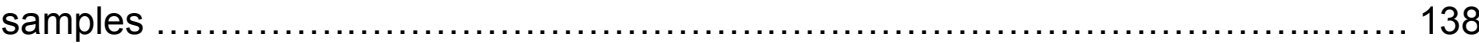

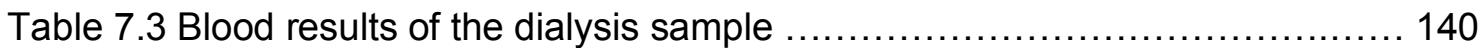

Table 7.4 Comparison of the findings between the demographic variables and SF-36

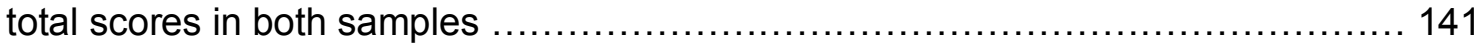

Table 7.5 Correlation between demographic data and SF-36 total scores ........... 143 Table 7.6.Clinical variables correlations with SF-36 total scores for the dialysis sample 143

Table 7.7 Comparison of the findings between the demographic variables and QOL

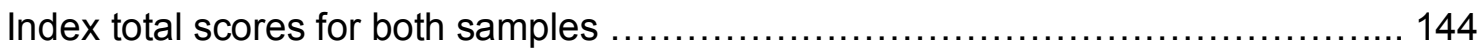

Table 7.8 Correlation between demographic data and QOL Index total scores ......... 145

Table 7.9 Lab values correlations with total scores QOL Index ....................... 146

Table 7.10 Significant findings of the SF 36 and QOL Index for the dialysis sample 147

Table 7.11 Multiple regression analysis of SF-36 total scores with demographic variables for dialysis sample

Table 7.12 Multiple regression analysis of SF-36 total scores with dummy categorical variables for dialysis sample 149

Table 7.13 Multiple regression analysis of total SF-36 scores with the clinical variables for dialysis sample 150

Table 7.14 Multiple regression analysis of QOL Index total scores with the demographic variables for dialysis sample 151

Table 7.15 Multiple regression analysis of QOL Index total scores with dummy variables for dialysis sample 152

Table 7.16 Multiple regression analysis of QOL Index total scores with clinical variables for dialysis sample 153

Table 7.17 Multiple regression analysis of SF-36 total scores with demographic variables for community sample 154

Table 7.18 Multiple regression analysis of SF-36 total scores with dummy variables for community sample 155

Table 7.19 Multiple regression analysis of QOL Index total scores with the demographic variables for community sample 156

Table 7.20 Multiple regression analysis of QOL Index total scores with dummy variables for community sample 157

Table 7.21 MANOVA tests for dialysis sample 159 
Table 7.22 MANOVA tests for the community sample for SF36 and Quality of Life Index 160

Table 8.1 Comparison between the SF-36 subscales and the total scores of both samples and the results from Kao et al. (2009) study 165

Table 8.2 Comparison between dialysis samples of the UAE study with international studies 166

Table 8.3 Comparison of the normative values of the SF-36 between UAE, Turkey, and Jordan

Table 8.4 Comparison of the subscales in both tools 183

Table 8.5 Significant variables in multiple regression analyses by tool and sample 185 


\section{List of figures}

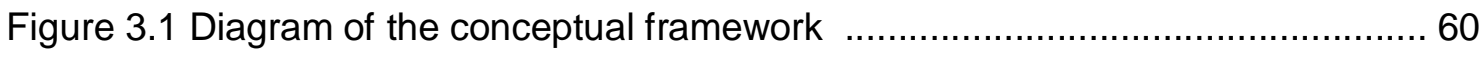

Figure 5.1 Normality plot of the combined SF-36 total scores ............................... 97

Figure 5.2 Normality plot of the combined QOL Index total scores ........................... 98

Figure 5.3 Normal Q-Q plot for the combined SF-36 total scores ............................ 99

Figure 5.4 Normal Q-Q plot for the combined QOL Index total scores $\quad . . \ldots \ldots \ldots \ldots \ldots \ldots . . . . . . . . .99$ 


\section{List of abbreviations}

Adaptation Training Programme: (ATP)

Blood Urea Nitrogen: (BUN)

Erythropoietin: (EPO)

End stage renal disease: (ESRD)

European Organization for Research and Treatment of Cancer: (EORTC)

Jalowiec Coping Scale: (JCS)

Kidney Disease Quality of Life: (KDQOL)

Malnutrition-Inflammation Complex Syndrome: (MICS)

Mental Health Component: (MHC)

Multivariate Analysis of Variance: (MANOVA)

National Kidney Foundation's Kidney Disease Outcomes Quality Initiative: (NKFK/DOQI)

One-way analysis of variance: (ANOVA)

Physical Health Component: (PHC)

Quality of Life: (QOL)

Quality of Life Index: (QOL Index)

Research and Development Corporation: (RAND Corporation)

Short Form Health Survey: (SF-36)

Sheikh Khalifa Medical City: (SKMC)

Statistical Package for Social Sciences: (SPSS)

Strengthening the Reporting of Observational Studies in Epidemiology: (STROBE)

United Arab Emirates: (UAE)

United States of America: (USA)

United Kingdom: (UK)

Urea Reduction Ratio (URR) 


\section{Chapter 1 Introduction and overview}

Quality of life (QOL) is an important parameter that needs to be considered when evaluating the experience and outcome of patients receiving healthcare. This is especially the case for patients with long term chronic diseases, since complete cure from their illness is often impossible (Macduff, 2000). There is an increasing interest in measuring QOL in both clinical trials and everyday clinical practice. In addition to mortality and morbidity as key indicators for performance, QOL has recognised as an important factor for evaluating the quality and outcome of healthcare for patients with chronic illnesses such as multiple sclerosis, asthma and chronic kidney failure. The acknowledgment that the burden of chronic kidney failure expand beyond its impact on the biological structure of the body is reflected in initiatives from the National Kidney Foundation in the United States of America (USA) to support efforts aimed at improving QOL in patients with kidney failure (K/DOQI, 2002).

Patients with chronic kidney failure have to receive dialysis therapy routinely for survival. Living on dialysis creates uncertainty about the future. These patients have to deal not only with treatment-related complications such as left ventricular hypertrophy, arthrosclerosis and hyperparathyroidism (Drueke \& Eckardt, 2002; London et al., 2001), but also with changes in their concept of self and self-confidence, and sometimes a reversal in family roles (Lev \& Owen, 1998). The major psychological and physiological stressors experienced by dialysis patients are pain, restriction of fluids, itching, discomfort, limitations in physical activity, fatigue, weaknesses, paying for the care, feelings of inadequacy and negative moods (Lok, 1996; Mok \& Tam, 2001; Welch \& Austin, 2001). In the USA, the annual mortality rate of this patient population is $15.17 \%$, a rate less than that seen in non-Hodgkin's lymphoma (23\%), ovarian cancer $(21 \%)$, colorectal cancer (17\%), and higher than patients with breast cancer $(2 \%)$ and prostate cancer (0.2\%) (USRDS Annual Data Report, 2010). Furthermore, a dialysis schedule can significantly obstruct both professional and personal lifestyle (Kimmel, 1995; Valderrabano, Jofre, \& López-Gómez, 2001). These factors may contribute to the QOL reported by patients on regular dialysis (Kimmel; Lopes et al., 2002; Merkus et al., 1999; Valderrabano et al.).

There is an increasing need for international standards to measure QOL in a manner that allows comparisons across cultures. The international standards have to be relevant to individual cultures. The known differences between Western and Eastern cultures may be reflected in the QOL measurement results (Tseng, Lu, \& Gandek, 
2003). To address this diversity, researchers have to take care when translating established instruments (Goh et al., 1996). QOL tools measure an individual's physical and mental state, completing then requires respondents to answer sensitive questions of items which can reflect differences in ethnicity and culture, even if the language used is the same in a broad sense. Because of differences in culture among countries speaking the same language, several versions of the SF-36 been developed to address the cultural differences in perceptions of health and illnesses in different countries. Although most of the words are similar, there are several English versions of QOL tools such as the SF-36 US English, UK English, Australian English, Canadian English, reflecting linguistic differences in the different countries. Furthermore, there are at least seven Arabic translated versions of the SF-36 tool (Algerian, Tunisian, Morocco, Egyptian, Jordanian, Lebanese, Saudi Arabian) (Quality Metric tools, 2011). The differences in the values and ways of living between countries necessitate having more than one version either in the English or the Arabic translated versions of QOL tools.

The aims of this study were to:

- $\quad$ Establish what is important in respect to the QOL for people living in the UAE

- Compare the cultural relevancy of two QOL tools between dialysis patients and a sample of the community living in UAE.

- $\quad$ Study the differences of QOL between dialysis patients in UAE and a sample of the general population

- Identify the physical, psychological, social, cultural, religions, gender, ethnicity influences and impacts on the QOL of people undergoing dialysis and a ample of the general population

- Examine the impact of dialysis adequacy and nutritional status on the QOL of dialysis patients

This study developed and trialed a new model to examine the cultural relevancy of two QOL tools and compared them to identify which tool was more culturally relevant. The following steps were used to examine the cultural relevancy of the tools. Firstly, this research studied and compared the QOL in dialysis patients with a sample from the community. Secondly, this study asked participants about their views of the cultural relevancy of the tools and asked participants to add or delete questions from the tools to make them culturally relevant. Thirdly, it examined the missing data to explore whether respondents missed answering questions due to evidence of non-applicability 
or cultural irrelevancy. Fourthly, this research included a qualitative section, in which three open-ended questions were asked to explore the views of both samples about what the people living in the UAE value in life. These questions asked respondents what they thought other people in the community value most in life, what they personally value most in life and what are the important things that maintain or improve their QOL.

This chapter outlines the background information about the study, provides a brief description of the UAE and highlights the significance of the study. It also describes the research questions, method, samples, tools and analysis plan, and introduces the researcher's interest and experience in the topic. Finally, it describes the structure of the thesis and lists the study contributions to nursing knowledge.

\subsection{Background of quality of life}

The term QOL in dialysis patients started appearing in the literature in the 1970s. There were limited approaches used to study QOL at that time. Most studies were conducted in USA, Canada, United Kingdom (UK) and the Netherlands. Since the 1970s a large number of research studies have been conducted using a variety of research designs including qualitative and quantitative approaches. The majority of quantitative studies have used different tools to study QOL. Moreover, these studies used various sociodemographic and clinical variables to study QOL in dialysis patients. Looking at how other researchers examined the different variables that impacted on QOL, helped in identifying the literature gaps and has informed this research design. This study is among the few that has compared two QOL tools on the same population, and is also the first known study on QOL in the UAE. Given what is known about the culture, religion and other social characteristics of this population, it was important to use two tools to study the QOL in this population. These two tools were developed in western countries and had been used with different population groups from all around the word. Both tools have different subscales and well documented validity and reliability studies. However, this study examined the cultural relevancy of those tools for people who live in UAE.

Despite considerable interest and multiple studies, the definition of QOL is still debated. QOL is often used to describe a variety of physical and psychosocial variables. Therefore, QOL is often used as a collective term covering a variety of concepts; such as functions, health status, perceptions, life conditions, behaviours, happiness, lifestyle 
and symptoms (Simko, 1999). The World Health Organization (WHO) QOL Group defined QOL as "an individual's perception of their position in life in the context of the culture and value system in which they live and in relation to their goals, expectations, standards, and concerns" (Orley, 1994, p.99). Orley suggests defining QOL as a person's perceptions of their own QOL in terms of being physically healthy, psychologically stable (free from anxiety and depression), having social support and acceptance in the community, being financially able to manage living a reasonable life fulfilling his or her family, marital, social, and cultural demands.

QOL represents patients' personal satisfaction with their life circumstances and can be influenced by how they respond to the physical, mental and social effects of illness on their daily living (Lukkarinen \& Hentinen, 1998). QOL is individualised, people respond differently to the same event (Hudson, 1995). A condition may be considered as an irritation for one person but may be severely frustrating for another (Ferrans, 1996). QOL in dialysis patients from different ethnicities and religious beliefs has been extensively researched and found to be significantly different (Hicks, Cleary, Epstein, \& Ayanian, 2004; Kimmel, Emont, Newmann, Danko, \& Moss, 2003; Patel, Shah, Peterson, \& Kimmel, 2002; Unruh et al., 2004). Given the importance of QOL it is essential to have measures that are able to capture patients' experiences of health, illness and treatment. Once individuals experience a decline in their health, their ability to function can affect their socio-economic status and marital relationships, consequently leading to a worsening QOL (Dunn, Lewis, Bonner, \& Meize-Grochowski, 1994).

Nurses are encouraged to learn how to work with patients from different cultures and New Zealand is one of the countries that promoted the teaching of culturally safe practice and inequities in access to quality health care for indigenous people (Peiris, Brown, \& Cass, 2008). Nurses should utilise cross cultural knowledge and culturally sensitive skills in implementing culturally congruent nursing care (Ulrich, 2009). Nurses are the centre of care, and they are concerned with maximising good patient outcomes. Also, they are the closest to the patients. They spend more time with patients than any other healthcare personnel. Nurses need to understand that QOL is critical in improving nursing practice. Nurses need to be aware of the cultural and the psycho-social issues related to QOL. As nurses need to be culturally safe in their practice (Lowe \& Archibald, 2009) researchers need to be culturally safe and aware in their research practices. This awareness must not only be in how nurses relate to patients but in how 
they collect data from their patients about different aspects of care. In UAE and other countries nephrology nurses should concentrate on developing clear concepts that look into psychosocial aspects of care such as emotional status, social involvement, productivity and employment. At the same time they should also focus on the practical application of these concepts.

\subsection{The United Arab Emirates}

The UAE (or the Emirates) is a Middle Eastern country situated in the South-East of the Arabian Peninsula, in the South-West of Asia on the Persian Gulf. It borders Oman and Saudi Arabia, and is comprised of seven emirates: Abu Dhabi, Ajman, Dubai, Fujairah, Ras al-Khaimah, Sharjah and Umm al-Quwain. The Emirates extends along part of the Gulf of Oman and southern coast of the Arabian Gulf. Before 1971, they were known as the Trucial States or Trucial Oman, in reference to the $19^{\text {th }}$ century truce between Britain and several Arab Leaders (Wilkinson, 2009).

UAE is a mix of cultures and ethnicities. The population of the UAE was 4,104,695 according to the 2005 census with only $11 \%$ being UAE Nationals. Other Arabic Nationals make up $21 \%$, South Asians make up $57 \%$ and people from western nations make up the remaining $11 \%$ (UAE Census, 2005). The UAE's superior living standards and economic opportunities have made it an attractive destination for Indians, Pakistanis, and many from Bangladesh and Sri Lanka. In 2006, the largest expatriate community were Indian Nationals (1.2 million) and there were 700,000 Pakistani Nationals; forming close to $60 \%$ of the population (Pejman, 2007). The UAE is governed by UAE Nationals. The dominant and official religion is Islam. Although no official figures are available, it is estimated that approximately $55 \%$ of the population are Muslim, 25\% are Hindu, 10\% are Christian, 5\% Buddhist, and 5\% belong to other religions, including Baha'i and Sikh (International Religious Freedom Report, 2005).

In Islamic communities, religion is a way of life that governs people's behaviour. Religion serves as a guide to people on how they should perceive the world around them especially different aspects of health. Muslims views regarding health, illness and death incorporate the notion of receiving these conditions with patience, meditation and prayers. For Muslims illness, suffering and dying are considered part of life and a test from Allah (God). Consequently, the way Muslim people in UAE perceive their own QOL might be different from others. 
Given that no studies were found in the literature examining the $\mathrm{QOL}$ in dialysis patients from UAE; it was very important to study and compare the QOL between a sample of dialysis patients and a group from the community from the same country. Studying the impact of religion on how UAE dialysis patients and other residents of UAE perceive QOL is important. Nurses and other healthcare professionals deal with several issues pertinent to QOL in dialysis patients almost on daily basis, yet little is known about the determinants of QOL in dialysis patients from UAE.

\subsection{QOL and dialysis patients}

Normal kidneys have two major functions; excretory and secretory functions. The excretory functions include; removal of excessive fluids in a form of urine, excretion of waste products such as urea and creatinine, maintain acid base balance and regulate electrolytes (Nettina, 2006). The secretory functions of the normal kidneys are secretion of erythropoietin to activate the bone marrow to produce red blood cells to regulate haemoglobin level, secretion of rennin to regulate blood pressure, and secretion of vitamin $\mathrm{D}$ to regulate calcium absorption from the intestine (Nettina).

Chronic renal failure is a gradual and progressive loss of kidney function. Diabetes and hypertension account for two thirds of the causes of end stage kidney failure (Jaber \& Madias, 2005). Patients may not develop symptoms until after more than $75 \%$ of glomerular filtration is lost; then the functions of the remaining nephrons deteriorate causing worsening symptoms (Springhouse, 2005). Symptoms of kidney failure affect all body systems; initially, salt-wasting that leads to hyponatremia produce hypotension, dry mouth, loss of skin turgor, fatigue and nausea. In later stages, irritability and confusion develop. Further loss of functioning nephrons reduces the kidneys' ability to excrete sodium, resulting in salt and water retention leading to fluid overload. Furthermore, accumulation of potassium causes muscle irritability and weakness as the potassium level continues to raise cardiac arrhythmias and possible cardiac arrest can occur (Springhouse). Dialysis is one of the treatment options for people with kidney failure in addition to kidney transplantation.

The life of patients treated with dialysis is characterised by many losses and restrictions. Patients need to restrict their food and fluid intake and avoid exerting jobs. In addition, patients need to comply with a difficult medication regime that includes taking many drugs. Some of these drugs should be taken with food, others after food or before food. Advancement in medical technology has extended the average life of 
patients with chronic illnesses (Goyen \& Debatin, 2009). Longer life expectancy for people with chronic health conditions can lead to poor QOL (Graham et al., 2009). Dialysis is accompanied by significant morbidity and mortality. Many physical and psychological symptoms occur in patients on chronic dialysis (Valderrabano et al., 2001). As many as $87 \%$ of dialysis patients have fatigue, up to $75 \%$ have skin itching and nearly $20 \%$ report they suffer from depression (Lopes et al., 2003; Merkus et al., 1999). Moreover, of the 263 deaths among New Zealand dialysis dependent patients in $2003,40 \%$ were due to cardiovascular causes, $26 \%$ to withdrawal from treatment, $11 \%$ to infection, and 5\% to malignancy (ANZDATA Registry Report, 2004). The ANZDATA Registry Report did not mention the number of dialysis patients with depression and/or adjustment disorders which are common among people on dialysis.

The literature on QOL in dialysis patients reveals that their QOL is highly affected as they are exposed to major physical, psychosocial and financial stressors. These include diet and fluid intake restrictions, potential losses and lifestyle changes, permanent invasive procedures like insertion of central venous lines and creation of multiple vascular accesses. They also have poor survival rates, weight loss, poor skin integrity, diminished muscle tone, oedema, pallor and constant fatigue, marital strain and sexual dysfunction, uncertainty, decreased self-confidence, reversal in family roles and loss of dignity. Some of them experience depression; life dissatisfaction, altered self-image, family responsibilities and social lives, financial dependence and unemployment. The dialysis schedule can also significantly obstruct both professional and personal lifestyle (Kimmel, 1995; Lopes et al., 2002; Merkus et al., 1999; Valderrabano et al., 2001). These factors may contribute to the impaired QOL reported by patients on maintenance dialysis. Nurses need to be aware of the QOL perceived by dialysis patients (Dunn et al., 1994). All the above factors can influence the QOL of dialysis patients, and can also affect the family members (Cook, 1995; Hudson, 1995).

\subsubsection{Overview of dialysis units in UAE}

Due to the lack of dialysis registry data in UAE, the researcher called all hospitals in UAE to obtain information about their dialysis units. After surveying all hospitals, 10 government hospital dialysis units and five private hospital dialysis units were found in UAE. A summary of statistics for the dialysis units in UAE is presented in Table 1.1.

Sheikh Khalifa Medical City (SKMC) had the largest number of patients followed by Dubai Hospital. Three dialysis centres used High Flux dialysis while the rest of the 
centres used Low Flux dialysis. The researcher was unable to get the yearly growth rate in the number of new patients. The number of hemodialysis patients is more than 18 times the number of patients on peritoneal dialysis and $11.6 \%$ of hemodialysis patients are on twice weekly dialysis.

Table 1.1 Summary of dialysis patient data for the dialysis units in UAE

\begin{tabular}{|c|c|c|c|c|c|c|}
\hline Unit names & ¿ & 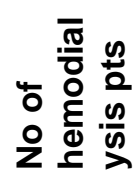 & 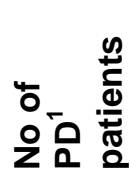 & 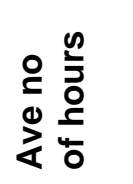 & 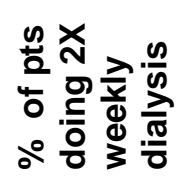 & 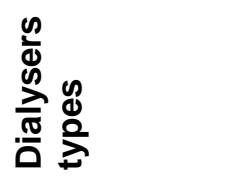 \\
\hline Sheikh Khalifa Medical City & 225 & 200 & 25 & 4 & $10 \%$ & $\begin{array}{r}\text { High \& low, } \\
\text { Flux }\end{array}$ \\
\hline Al Rahba Hospital & 22 & 22 & 0 & 4 & 0 & High flux \\
\hline Tawam Hospital & 147 & 147 & 0 & 4 & $20 \%$ & Low flux \\
\hline Zayed City Hospital & 15 & 14 & 1 & 4 & $15 \%$ & High flux \\
\hline Dubai Hospital, & 195 & 180 & 15 & 4 & $5 \%$ & Low flux \\
\hline Al Qassimi Hospital & 110 & 110 & 0 & 4 & $8 \%$ & Low flux \\
\hline Fujerah Hospital & 31 & 31 & 0 & 4 & $10 \%$ & Low flux \\
\hline Kalba Hospital & 8 & 8 & 0 & 4 & 0 & Low flux \\
\hline Deba Hospital & 6 & 6 & 0 & 4 & $3 \%$ & Low flux \\
\hline Umm Al Qewin Hospital & 16 & 16 & 0 & 4 & 0 & Low flux \\
\hline American Hospital & 7 & 7 & 0 & 4 & $40 \%$ & Low flux \\
\hline Welcare Hospital & 14 & 14 & 0 & 4 & $10 \%$ & Low flux \\
\hline Belhoul Hospital & 7 & 7 & 0 & 4 & $30 \%$ & Low flux \\
\hline Total & 803 & 762 & 41 & & & \\
\hline
\end{tabular}

1. PD means peritoneal dialysis

\subsection{The research process}

This research was carried in Abu Dhabi, the capital city of the UAE. The dialysis arm of the study was carried out with patients from the Sheikh Khalifa Medical City (SKMC) renal programme. This programme is the largest in the UAE and provides a range of services to patients with kidney diseases including treatment of common kidney disorders, before and after kidney transplant preparation and follow-up, hemodialysis, peritoneal dialysis and paediatric dialysis. In 2007, the total number of patients at SKMC with post kidney transplant was approximately 300 and the hemodialysis patients were 192, the number of peritoneal dialysis patients was 28 and there were four paediatric dialysis patients (Renal Unit at SKMC Annual Statistics, 2007). The 
community arm of the sample comprised 350 participants chosen randomly from different areas in Abu Dhabi.

Prior to commencement of the study, approvals were obtained from the Human Ethics Committee at Victoria University of Wellington and SKMC Ethics Committee (Appendix A \& B). A descriptive comparative survey design was used. Between May and July 2007, 155 dialysis patients and 350 participants from the community were invited to participate in this study. The QOL tools used in this research were (SF-36 for both samples and QOL Index Dialysis version for the dialysis sample and QOL Index generic version for the general population sample). The SF-36 consists of 36 items that assess eight dimensions (subscales) of QOL: physical functioning, role-physical functioning, bodily pain, general health, vitality, social functioning, role-emotional functioning, and mental health. The QOL Index consists of a 34-item measure of satisfaction with various dimensions of life and the importance of the same dimensions. It is composed of five subscales: health and functioning, social and economic, psychological and spiritual, and family. A conceptual framework was developed by the researcher to inform data interpretation. The Statistical Package for Social Sciences (SPSS) for Windows version 13.0 (SPSS Inc, Chicago, IL, USA) software was used for data analysis.

The analysis plan was designed to generate answers for each of the research questions. Clinical and demographic variables were described using descriptive statistics such as mean, percentage or standard deviation. The relationship between total scores of SF-36 and QOL index (dependent variables) and demographic data (independent variables) were described using measures of central tendency and dispersion such as mean, standard deviation, median and frequencies. Multiple linear regression analysis was performed to determine variables that might affect the QOL scores. Also to assess the ability of the independent variables to predict levels of total scores QOL tools, Multivariate Analysis of Variance (MANOVA) of both samples on the dependent variables were undertaken. Content analysis was used to analyse the findings from the qualitative data gathered from the open-ended questions at the demographic section of the questionnaire to identify any QOL determinant that might not be captured by the questionnaires. 


\subsection{The researcher and the topic}

I am a New Zealand Muslim of Middle Eastern descent. I have lived and worked in several countries in the Middle East and New Zealand. The QOL of dialysis patients is one of my most favoured topics that I often discuss with fellow healthcare professionals. My experience around dialysis started 16 years ago when I worked as a Staff Nurse in the Renal Unit at Jordan University Hospital. This experience was enriched between 2002 and 2005 when I was enrolled in a Master of Nursing through Massey University, New Zealand. The Master Programme was geared toward becoming a Dialysis Nurse Practitioner with Prescribing Right. While studying the Master programme I worked as a Dialysis Resource Nurse at Waikato Hospital in Hamilton, New Zealand. During this period I managed to work closely with dialysis patients through the Home Hemodialysis Training Programme in which I dealt with different psycho-social issues as well as family dynamics of hemodialysis patients. More recently, I worked as a Clinical Resource Nurse in the dialysis unit at SKMC (the location of this research). In this role, I was responsible for educating around 60 nurses and 192 patients on different topics related to dialysis. Having nursed people on dialysis in three different countries (Jordan, New Zealand and UAE) it was apparent that people on dialysis coped with their illness differently. Throughout my observation in these countries QOL was rarely discussed in the ward rounds or amongst the nursing staff themselves. All of those factors shaped my ideas around this research.

In summary, this research

- Established what is important in respect to the $\mathrm{QOL}$ for people living in the UAE

- Studied the differences of QOL between dialysis patients in UAE and a group of the general population

- Identified the physical, psychological, social, cultural, religious, gender, ethnicity influences and impacts on the QOL of people undergoing dialysis and a group of the general population

- Examined the impact of dialysis adequacy and nutritional status on the QOL of dialysis patients

- Tested and compared the cultural relevancy of two QOL tools between dialysis patients and a group of the community living in UAE. 


\subsection{Structure of the thesis}

This first chapter has set the scene for this research. Chapter 2 describes the search strategy used to identify the literature related to $\mathrm{QOL}$ in general and specifically to dialysis patients. It also discusses what is understood about $\mathrm{QOL}$ and how it has been researched. This chapter defines culture and explains the importance of understanding how culture impacts on QOL. This literature review around the QOL concept is framed using six conceptual debates inherent to the notion of QOL as outlined by Moons, Budts and De Geest (2006). Furthermore, this chapter critically reviews the literature to clarify the demographic and clinical factors that contribute to the QOL in dialysis patients.

Chapter 3 explores the relationship between culture and QOL tools. It also discusses the importance of assessing the cultural relevancy of QOL tools and examines the methods of translations and cross-cultural adaptation of QOL tools. Also this chapter presents an overview of general QOL tools used in studying QOL and describes and critiques the QOL tools used in this research. The conceptual framework that was developed to inform data collection and analysis for this research is explained in this chapter. It describes the interaction of different relevant variables on individuals' perceptions of their QOL such as culture and society, religion, physical health, family and social support, economic resources, and life events.

Chapter 4 describes the methodology used in this study including the advantages and disadvantages of the descriptive survey design and the sampling process. This chapter discusses the ethical issues and describes briefly the pilot study. It describes data entry, the management of missing data, and description of the survey participants and data distribution. Analysis of QOL tools, cultural relevancy of the tools, analysis of qualitative data and ranking of the themes, correlations and differences of sociodemographic and life factors with QOL total scores were described in this chapter. It describes the procedure for analysis of the multiple regression and multivariate analysis of variances.

Chapter 5 presents the overall return rates and level of missing data for both samples and describes the differences in the socio-demographic variables of both samples and missing data in both samples. This chapter also reports on and compares the cultural relevancy of both tools for the two samples. The last section presents and compares 
the results of the qualitative data and reports on the themes that emerged from the open-ended questions.

Chapter 6 discusses the samples' characteristics and representation, and compares the demographic similarities and differences of both samples. It discusses the cultural relevancy of the tools and examines the impact of missing data on the cultural relevancy of the tools. This chapter also explores the characteristics of the respondents who did not answer the question about satisfaction and importance of living as long as they would like to, and the satisfaction and importance of sex and spouse. Furthermore, this chapter discusses the themes that were identified from the analysis of the qualitative data.

Chapter 7 presents the total scores of both tools and their subscale scores, compares the results between both samples, and presents the findings from blood results for the dialysis sample. It also presents the findings of the correlation and the differences of the significant values in the total scores of QOL of the dialysis and community samples and describes the demographic differences and similarities between both samples. It also presents the results of the multiple regression analysis and the multivariate analysis of variance (MANOVA) of both samples.

Chapter 8 discusses the differences in the findings between the SF-36 and QOL Index tools for both samples. It also discusses the effect of predictor variables on the total scores of both tools compared with international studies as well as the correlation between other demographic variables and total scores of both tools. It compares the significant values of the two tools in both samples. Finally it discusses the findings from the multiple regression and the MANOVA analysis findings.

Chapter 9 summarises the major findings, examines the limitations of the study and presents the implications for practice and dialysis care as well as the recommendations for future research. 


\section{Chapter 2 Literature Review}

As part of determining how to examine $\mathrm{QOL}$ in the UAE, the literature related to $\mathrm{QOL}$ generally and in particular focusing on QOL for dialysis patients was examined. This chapter describes the search strategy used to identify the literature related to $Q O L$ in general and specifically to dialysis patients followed by a brief description of where the interest in studying QOL has come from and definitions of QOL. This chapter also discusses what is understood about QOL and how it has been researched. This discussion is framed using six conceptual debates inherent in the notion of QOL as outlined by Moons et al. (2006). This chapter examines the impact of culture on QOL. The final section focuses on what is known about the demographic and clinical factors that impact on the QOL in dialysis patients. The relevant literature about QOL tools in general will be presented in the next chapter.

\subsection{Search strategy}

Computerised literature searches were performed, with language restriction to English, using Psych-Info, Medline, Pro-Quest with multiple database options and Wiley Interscience Databases 1980-2010. The PubMed search of Medline involved the use of medical subject headings as well as text word searching using the terms: quality of life concept, renal insufficiency, end stage renal disease, chronic kidney failure, quality of life and quality of life tools. These terms were combined with the terms age, gender, ethnicity, religion, employment, education, marital status, length of time on dialysis, chronic illness, knowing the cause of kidney failure, family, social support, self-image, haemoglobin and anaemia, dialysis adequacy, serum albumin, and length of time on dialysis. The search was not limited to particular countries. Several research studies discussed QOL in dialysis patients and community samples, but no search was undertaken to find research on the $\mathrm{QOL}$ in community samples only. Articles that described opinions about the QOL of dialysis patients were excluded and only primary research studies that used rigorous scientific scholarly research, such as peerreviewed studies were included in the literature review. The relevant articles were studied for their research methodology, sample size, QOL tool used, variables studied, the response rate, major findings and conclusions. Studies were compared for similarities and contradictions in findings and the limitations of the studies were examined.

Several research designs were examined for their suitability to answer the research questions including both qualitative and quantitative. A decision was made that the 
most suitable design that could answer the research questions was descriptive survey design. The descriptive approach would describe the QOL in relation to other variables using quantitative and qualitative methods. In the quantitative part, two QOL tools were used (SF-36, QOL Index tool) to describe the QOL of between dialysis and compare it with QOL of group from the community. The survey approach was the best and doable design to research people from different cultural backgrounds speaking different languages. The researcher speaks two languages (English and Arabic).

\subsection{The notions or interest of studying QOL concept}

QOL has been considered an important factor in the examination of patient responses to illness and treatment. In the past two decades the concept of QOL has increasingly been applied to study and evaluate the health conditions of patients with chronic illnesses (Schalock, 2004). Schalock emphasised that the interest in QOL has come from four sources. Firstly, there has been a shift from the belief that advances in medical technology alone would result in improved QOL, toward an understanding that personal, family, community and societal well-being are also important. Secondly, the interest is a logical step from the normalisation movement that stressed communitybased services to assess and measure the outcomes of improved individuals QOL in the community. Thirdly, the increased emphases on customer empowerment and patients' rights have led to a focus on patient-centred care of which QOL is an element. Fourthly, the appearance of sociological research has introduced the subjective or perceptual aspects of QOL and the individual characteristics involved, generating the need for further research. The increased interest for studying QOL has therefore emerged from a necessity to include patients' empowerment, family involvement and support as well as the availability of community based services.

\subsubsection{Definition of QOL}

There is no consensus regarding the definition of QOL in the literature. Some argue that there is more disagreement on what QOL means than on any other concept in medical, social and psychological research (Dijkers, 2005; Gill \& Feinstein, 1994; Veenhoven, 2000). Despite the literature that has been dedicated to the measurement of $\mathrm{QOL}$, there is also no one way of constructing and measuring QOL (Berzon, Donnelly, Simpson, Simeon, \& Tilson, 1995; Gill \& Feinstein; Sijtsma, Emons, Bouwmeester, Nyklíček, \& Roord, 2008). Gill and Feinstein examined 75 articles describing the use of QOL instruments and found that only $15 \%$ of the articles attempted to define what was meant by QOL or to justify the choice of QOL measures. 
None of these articles distinguished clearly among QOL, health-related QOL and subjective well-being.

Conceptually, most researchers agree that QOL is multidimensional consisting of a number of subjective and objective dimensions (Ferrans, 1990a; Meeberg, 1993; Peplau, 1994; Zhan, 1992). Muldoon, Barger, Flory and Manuck (1998) believe that measuring the subjective and objective dimensions of $\mathrm{QOL}$ and applying this in practice will make the definition clearer and more precise because each dimension contributes to an overall assessment of QOL. Dijkers (2005) proposed a comprehensive definition when he considered that each person has bio-psycho-sociocultural needs, desires, priorities and standards. When these needs, desires and priorities are compared by people to their personal situation, reactions can range from positive to negative and be reflected in people assessment of their morale and life satisfaction. These reactions comprise one (but perhaps the most complete) definition of QOL: "the sum total of the cognitive and emotional reactions that people experience when they compare what they have and do in life with their aspirations, needs and other expectations" (Caiman, 1984). Other definitions of QOL are summarised in Table 2.1.

Defining QOL in terms of satisfaction with life is most appropriate. It reflects the degree to which a person positively evaluates the overall subjective and objective components of his or her life. Furthermore, QOL refers to the level of enjoyment and satisfaction with the life led so far. Therefore the appraisal of satisfaction with life is preceded by a cognitive evaluation of one's personal life conditions; this indicates that patients should judge the quality of their own life. Thus, in this respect, satisfaction with life differs from happiness/affect, which is more emotion driven. QOL has been developed as a universal concept that is affected by multiple factors. Consequently, it is considered that individuals are the only ones who can reliably estimate their own QOL (Ferrans, 1996). An individual's circumstances, culture and religion have an effect on it (Patel et al., 2002). The QOL tools (SF-36 and QOL Index) have been used extensively in researching people from different countries, but to date there are no published research studies that used these tools in the United Arab Emirates. 
Table 2.1 Examples of definitions of QOL

\begin{tabular}{|l|l|}
\hline Source & Definition \\
\hline $\begin{array}{l}\text { Edlund \& Tancredi } \\
\text { (1985) }\end{array}$ & One's ability to lead a socially "useful" life \\
\hline Ferrans (1992) & $\begin{array}{l}\text { The balance between positive feelings (elation) and negative feelings } \\
\text { (depression) }\end{array}$ \\
\hline $\begin{array}{l}\text { Janssen Quality-of- } \\
\text { life Studies (2006) }\end{array}$ & $\begin{array}{l}\text { A subjective well-being. Recognising the subjectivity of QOL is a key to } \\
\text { understand this construct. QOL reflects the difference, the gap, between } \\
\text { the hopes and expectations of a person and their present experience. }\end{array}$ \\
\hline $\begin{array}{l}\text { QOL Research } \\
\text { Unit, University of } \\
\text { Toronto (2006) }\end{array}$ & $\begin{array}{l}\text { The degree to which a person enjoys the important possibilities of his/her } \\
\text { life. Possibilities result from the opportunities and limitations each person } \\
\text { has in his/her life and reflect the interaction of personal and environmental } \\
\text { factors. }\end{array}$ \\
\hline $\begin{array}{l}\text { Quality-of-Life } \\
\text { Research Center, } \\
\text { Denmark (2006) }\end{array}$ & $\begin{array}{l}\text { Subjective QOL is about feeling good and being satisfied with things in } \\
\text { general. Objective QOL is about fulfilling the societal and cultural } \\
\text { demands for material wealth, social status and physical well-being. }\end{array}$ \\
\hline Leidy (1994) & $\begin{array}{l}\text { The ability to supply basic needs and to maintain health and well-being } \\
\text { Ontario for community development which can be used to monitor key } \\
\text { indicators that encompass the social, health, environmental and economic } \\
\text { dimensions of the QOL in the community }\end{array}$ \\
\hline $\begin{array}{l}\text { Development } \\
\text { Council (1997) }\end{array}$ & $\begin{array}{l}\text { QOL is a perception that encompasses the prevailing view of one's life at } \\
\text { a particular point in time }\end{array}$ \\
\hline Peplau (1994) & $\begin{array}{l}\text { An individual's perception of their position in life in the context of the } \\
\text { culture and value system in which they live and in relation to their goals, } \\
\text { expectations and standards and concerns. It is a broad ranging concept } \\
\text { affected in a complex way by the person's physical health, psychological } \\
\text { state, and level of independence, social relationships, and their } \\
\text { relationship to their environment. }\end{array}$ \\
\hline $\begin{array}{l}\text { WHO Quality of } \\
\text { life-BREF (2006) }\end{array}$ \\
\hline
\end{tabular}

\subsection{Researching QOL}

This section uses the classifications of Moons et al. (2006) to examine and critique how QOL has been researched. Moons et al. critiqued the conceptualisations of QOL by reviewing and evaluating different conceptual approaches. They presented an overview and critiqued conceptualisations of the QOL to make QOL a less ambiguous concept. They elaborated on Kinney's (1995) critique and described six conceptual aspects inherent to the notion of QOL. These aspects were used as criteria to evaluate the appropriateness of different conceptualisations.

\subsubsection{QOL as health status and functioning}

Researchers have studied QOL differently. Muldoon et al. (1998) stated that QOL research seeks mainly two kinds of information, functional status and impact of health on life quality. Some contemporary QOL instruments were designed so that the adequacy of individuals' functioning across life's physical dimensions could be related independently (QOL Research Unit, University of Toronto, 2006), and occupational and interpersonal dimensions (Lee, 2005). Published reports describing QOL instruments often use the terms health status, functional status and QOL interchangeably (Bradley, 
2001; Nanda \& Andresen, 1998; Stuifbergen \& Barbara, 2005), assuming that healthy life is equal to a high QOL. This interchange can be challenged because patients with health and functional problems may not necessarily have comparable QOL scores (Carr \& Higginson, 2001). Indeed, an empirical study (Garratt \& Ruta, 1999) and a meta-analysis (Smith, Avis, \& Assmann, 1999) found that QOL and health status are different concepts and recommend that they are not used interchangeably. Health remains the common aspect that is measured in almost all QOL tools. Therefore, this basic human experience needs to be addressed when assessing QOL. This study has compared a health-related QOL tool (SF-36) with a disease-specific tool (QOL Index) that also has a generic version, to explore the concept of QOL as health status and functioning in UAE population.

\subsubsection{Objective and subjective dimensions of QOL}

Objective dimensions refer to observable life conditions or physical functioning. Subjective dimensions refer to the respondent's perceptions. Objectivity is demonstrated when measuring a patient's ability to perform common tasks or activities such as climbing stairs, while subjectivity is demonstrated when asking patients to rate the effects of health status on personal wellbeing (Muldoon et al., 1998). Muldoon et al. believe that measuring the subjective and objective dimensions of QOL such as physical, psychological condition, family and friends, work, community, health, education and spiritual domains is important, and applying the subjective objective approach in practice will make the QOL concept clearer and more precise because each dimension contributes to an overall assessment of the QOL. The complementary, perspective on QOL allocates vital value to an individual's subjective appraisal of their health status. This approach presumes that QOL is partly independent of health status (Guyatt, Feeny, \& Patrick, 1993), and reflects the way how patients perception and response to their health conditions and to other non-medical aspects of their lives (Gill \& Feinstein, 1994).

Dijkers (2005) studied the concept of QOL by separating it into three major groups: subjective well-being, achievements, and utility. He interlinked the three concepts. The subjective well-being as identified by Dijkers (2003) consists of social standards and priorities. These standards and priorities impact on objective evaluation of the disability resulting from chronic illnesses. While the individual expectations and priorities are composed of goals, aspirations, values, standards, desires, needs and wants, these expectations and priorities impact on subjective wellbeing, life satisfaction, self-esteem, 
mood and happiness. The objective and subjective evaluations and reactions are expressed in the QOL in a form of achievements which include performances, possessions, relationships, accomplishments, characteristics and health (Dijkers, 2003). The utility aspects of QOL focused on productivity and employment as a social utility (Dijkers, 2005).

Joyce, Hickey, McGee and O'Boyle (2003) used a completed by patients open-ended questions to assess QOL. These questions allow respondents to choose the life dimensions that add most to their overall QOL from their perspective. The Schedule for the Evaluation of Individual QOL-Direct Weight (SEIQOL-DW) tool has been used with adult and elderly people geriatric including those with HIV/AIDS (Hickey et al., 1996), cancer (Waldron, O'Boyle, Kearney, Moriarty, \& Carney, 1999), serious mental illness (Prince \& Gerber, 2001), diabetes (Wagner, Abbott, \& Lett, 2004), hodgkin lymphoma (Wettergren, Bjorkholm, \& Langius-Eklof, 2005) and multiple sclerosis (Lintern, Beaumont, Kenealy, \& Murrell, 2001). This complementary approach allows respondents to focus on the dimensions in their life that they consider important. Resnicow and colleagues (2002) advocated for using what is valued by people to promote healthy behaviour and is consistent with a patient and family centred approach to medical care (Davis, Schoenbaum, \& Audet, 2005).

What constitutes QOL is debated in the literature; some researchers advocate for subjective dimensions while others advocate for objective dimensions and some advocate for both (Felce, 1997; Testa \& Simonson, 1996). There is no universal consensus on which life dimensions contribute most to overall QOL (Bishop \& Allen, 2003). However, the concepts that support the subjective and the objective dimensions are more dominate in research (Felce, 1997; Haas, 1999; Testa \& Simonson). However, there is a growing consensus that QOL is a purely subjective experience (Bishop, Chapin \& Miller, 2008) because it is unlikely that QOL is strongly determined by one's objective life condition, rather QOL is determined by one's subjective appraisal of one's life condition. Consequently, individuals are the only ones who can reliably estimate their own QOL (Ferrans, 1996).

Different people may place different emphasis on various aspects of their lives; one patient may consider the quality of family relationship to be the most important determinant of his or her QOL, whereas another may consider overall health or the ability to work to be far more important (Steele et al., 1996). It can be misleading to 
equate overall QOL with the sum of distinct objective functional items, whereas many earlier studies relied upon the healthcare professionals such as physician, nurse, social worker or psychologist to determine a person's QOL (Rebollo, Alvarez-Ude, Valdes, Estebanez, \& FAMIDIAL Study Group, 2005). Nurses and lay caregivers generally overestimate the psychosocial impact of a condition, while doctors consistently underestimate the severity of symptoms (Addington-Hall \& Kalra, 2001). There is often little agreement between patients and caregivers on the criteria for QOL. Kimmel and Patel (2006) have placed greater emphasis upon the patients' own assessments, functional or satisfaction level. This current study examined the subjective and the objective dimensions of QOL. Participants were given the opportunity to assess their QOL in a subjective manner using the QOL Index and in an objective manner using the SF-36. In addition, participants were also asked to describe three things that people living in UAE valued most in life, what they personally valued most in life and what were the most important things to them in maintaining or improving their QOL.

\subsubsection{Indicators and determinants of QOL}

Indicators are events or conditions that typically characterise a specific situation; they are "barometers". Determinants, on the other hand, are defined as elements that determine the nature of something and can therefore be considered as external factors that affect a phenomenon (Merriam-Webster online, 2009). The literature has identified several QOL indicators. The indicators are summarised in Table 2.2.

Several determinants have been studied to measure their impact on QOL. The most commonly studied determinants are age, gender, marital status, educational level, employment status and family support (Belasco et al., 2006; Bohlkeet al., 2008; Kutner et al., 2005; Maor, King, Olmer, \& Mozes, 2001; Moreno, Lopez-Gomez, SanzGuajardo, Jofre, \& Valderrabano, 1996; Neri et al., 2005). Other determinants that have been studied less often include religion, ethnicity, exercise, sleep, pain, sexual dysfunction, satisfaction with care, depression, symptom burden and impact of illness on daily life (Cerqueira, Moraes \& Glina, 2002; Gusbeth-Tatomir, Boisteanu, Seica, Buga, \& Covic, 2007; Hicks et al., 2004; Kimmel et al., 2003; Kimmel \& Patel, 2006; Kutner \& Devins, 1998; Patel et al., 2002; Pifer et al., 2003; Rabetoy, 2007; Tentori, 2008; Weinberg, Hoffmann, Pohle, Hampel, \& Schindler, 2007: Welch \& Austin, 2001). In dialysis patients, the determinants studied include haemoglobin level (BreitermanWhite, 2005) and modality of dialysis therapy (Rubin et al., 2004). There may be other determinants yet to be discovered that also impact on QOL. However, to what extent 
the variables listed above impact on people from Arabic and Islamic perspective is not known.

Table 2.2 Indicators of QOL

\begin{tabular}{|c|c|}
\hline Indicators & Description \\
\hline $\begin{array}{l}\text { Perceived QOL (Amarantos et } \\
\text { al., 2001) }\end{array}$ & $\begin{array}{l}\text { Subjective evaluation of both mental and physical status by the } \\
\text { respondent }\end{array}$ \\
\hline $\begin{array}{l}\text { Objectively assessed aspects } \\
\text { of the environment (Lee, } \\
\text { 2005) }\end{array}$ & $\begin{array}{l}\text { The physical environment and settings (e.g. home, nursing } \\
\text { home, hospital), are highly associated with QOL as are social } \\
\text { environments (e.g. living with relatives, alone). These aspects } \\
\text { are explored in this dimension }\end{array}$ \\
\hline $\begin{array}{l}\text { Human adaptation (Janssen } \\
\text { QOL Studies, 2006) }\end{array}$ & $\begin{array}{l}\text { Is such that life expectations are usually adjusted so as to lie } \\
\text { within the realm of what the individual perceives to be possible }\end{array}$ \\
\hline $\begin{array}{l}\text { Physical Being (QOL } \\
\text { Research Unit, University of } \\
\text { Toronto, 2006) }\end{array}$ & $\begin{array}{l}\text { Includes aspects of physical health, personal hygiene, nutrition, } \\
\text { exercise, grooming, clothing, and physical appearance }\end{array}$ \\
\hline $\begin{array}{l}\text { Psychological Being (QOL } \\
\text { Research Unit, University of } \\
\text { Toronto, 2006) }\end{array}$ & $\begin{array}{l}\text { Includes the person's psychological health and adjustment, } \\
\text { cognitions, feelings, and evaluations concerning the self, and } \\
\text { self-control }\end{array}$ \\
\hline $\begin{array}{l}\text { Spiritual Being (QOL } \\
\text { Research Unit, University of } \\
\text { Toronto, 2006) }\end{array}$ & $\begin{array}{l}\text { Reflects personal values, personal standards of conduct, and } \\
\text { spiritual beliefs which may or may not be associated with } \\
\text { organised religions }\end{array}$ \\
\hline $\begin{array}{l}\text { Physical Belonging (QOL } \\
\text { Research Unit, University of } \\
\text { Toronto, 2006) }\end{array}$ & $\begin{array}{l}\text { The connections the person has with his/her physical } \\
\text { environments such as home, workplace, neighbourhood, } \\
\text { school and community }\end{array}$ \\
\hline $\begin{array}{l}\text { Social Belonging (QOL } \\
\text { Research Unit, University of } \\
\text { Toronto, 2006) }\end{array}$ & $\begin{array}{l}\text { Includes links with social environments and includes the sense } \\
\text { of acceptance by intimate others, family, friends, co-workers, } \\
\text { and neighbourhood and community }\end{array}$ \\
\hline $\begin{array}{l}\text { Community Belonging (QOL } \\
\text { Research Unit, University of } \\
\text { Toronto, 2006) }\end{array}$ & $\begin{array}{l}\text { Represents access to resources normally available to } \\
\text { community members, such as adequate income, health and } \\
\text { social services, employment, educational and recreational } \\
\text { programs, and community activities. }\end{array}$ \\
\hline $\begin{array}{l}\text { Becoming (QOL Research } \\
\text { Unit, University of Toronto, } \\
\text { 2006) }\end{array}$ & $\begin{array}{l}\text { Refers to the purposeful activities carried out to achieve } \\
\text { personal goals, hopes, and wishes. Practical becoming } \\
\text { describes day-to-day actions such as domestic activities, paid } \\
\text { work, school or volunteer activities, and social needs. }\end{array}$ \\
\hline $\begin{array}{l}\text { Leisure (QOL Research Unit, } \\
\text { University of Toronto, 2006) }\end{array}$ & $\begin{array}{l}\text { Includes activities that promote relaxation and stress reduction. } \\
\text { These include games, neighbourhood walks, and family visits, } \\
\text { or longer duration activities such as vacations or holidays }\end{array}$ \\
\hline
\end{tabular}

There is a poor distinction between indicators and determinants of QOL (Stewart, 1992). Neri et al. (2005) considered employment as a determinant of QOL and Kimmel et al. (2003) referred to religion as a determinant of QOL. In contrast, the QOL Research Unit, University of Toronto (2006) referred to both variables as indicators of QOL. Lee (2005) considered living conditions as indicators, yet in contrast, Neri et al. referred to them as a determinant of QOL. The distinction between indicators and determinants is crucial for conceptualising QOL. In the conceptualisation, one needs to distinguish between indicators of QOL (e.g., what is QOL? What refers to QOL?) and determinants of QOL (e.g., What contributes to QOL? What influences QOL?) (Smith et 
al., 1999). For instance, an indicator for kidney failure is a significant rise in serum creatinine and urea levels, whereas determinants of kidney failure include age, race, diet and family history. From this example, it is obvious that the screening and diagnosis of kidney failure requires the assessment of the indicators such as haemoglobin and albumin levels urea reduction ratio, rather than the determinants of QOL such as age, ethnicity, marital status and employment. Given the inconsistency in the differences between indicators and determinants of $\mathrm{QOL}$, and the differences between the subjective and objective components of QOL, this research adopted a comprehensive approach by studying key indicators, determinants, and subjective and objective components of QOL in dialysis patients and a sample of healthy population in UAE using various methods of data collection such as structured QOL tools and openended questions.

\subsubsection{Negative and positive components}

There are many characteristics that can determine negative and positive components of QOL such as the coping skills used by patients with chronic illnesses. Coping ability has been suggested to influence one's sense of well-being and adaptation to illness (Weisbord et al., 2005). Family and social support play an important role in how people cope positively with chronic illness. Lindqvist et al. (2000) performed a descriptivecomparative study to delineate coping styles and health-related QOL among spouses of renal disease patients. Fifty-five patients were surveyed using a descriptive-co relational survey design. Perceived efficiency in coping with major aspects of the disease was studied using the Jalowiec Coping Scale (JCS) (Jalowiec, 1990). The most frequently used and effective coping style was the optimistic style. An obvious limitation of the Lindqvist et al. is that the design, does not demonstrate cause. The study used unequal proportions of husbands and wives in the haemodialysis and transplant groups, spouses were studied only once, and the samples were relatively small and non-randomly chosen.

QOL is primarily measured in terms of limitations and obstacles, without considering positive elements that contribute to it (Hyland, 1999). However, there are numerous positive things that can contribute positively to a person's QOL, as they add to life's richness. Even illnesses may be positively perceived. How people perceive and cope with the negative components of their life may vary among ethnic or cultural groups and from one person to another in the same culture. For example, studies of cancer patients showed that they have a better appreciation of each day, due to enhanced 
personal strength, self assurance and compassion (Fromm, Andrykowski, \& Hunt 1996; Tempelaar et al., 1989). One's positive personality or outlook toward life can also contribute positively to one's QOL. Therefore, the conceptualisation of QOL should clearly include both positive and negative factors. In this research, the positive and the negative components of QOL parts were addressed by using the QOL Index which asks people to specify their satisfaction and dissatisfaction with certain elements of their lives.

\subsubsection{QOL changes over time}

The impact of whether changes occur over time in QOL is debated in the literature. Many studies have indicated that QOL changes over time. Two studies have focused on the changes in QOL of individuals over time. Van Wyk, Vaz, Harries and Weighill (2008) assessed the impact of seasonal change (i.e. summer vs winter) on the QOL of patients following total laryngectomy. Their study of 36 patients who had recurrencefree laryngectomy using the European Organization for Research and Treatment of Cancer (EORTC QLQ-C30) Version 3 tool found that patients' responses were remarkably consistent and there were no statistically significant differences in scores between summer and winter in any of the EORTC QLQ-C30 tool dimensions. Merkus et al. (1999) studied the impact of dialysis modality, changes in physical and mental conditions on QOL using the SF-36 during the first 18 months of renal replacement therapy. QOL was assessed at baseline, 6, 12 and 18 months after the initiation of dialysis. The results of their study underscore the poor functional status of patients with ESRD. Merkus et al. found that the QOL among patients who died had deteriorated at a faster rate than the groups who lived. Moreover, significant differences were found between the groups with respect to age, co-morbidity index, cardiovascular comorbidity, diabetes mellitus, body mass index, albumin and haemoglobin levels. The sample size in the Merkus et al. study was 230 patients, considerably higher than the Van Wyk et al. study. Also, Merkus et al. studied five groups of dialysis patients over the four time periods after the initiation of dialysis while Van Wyk et al. studied the same patients over two periods of time (summer and winter).

QOL cannot be considered to be as a motionless feature (Carr \& Higginson, 2001). Individuals may appraise their QOL differently over time (Carr \& Higginson; Merkus et al., 1999) due to ever-changing life events, illness progress, coping abilities, or cultural circumstances. A patient's QOL changes, because of the demands of life that are important to an individual's QOL (O'Boyle, McGee, Hickey, O'Malley, \& Joyce, 1992) or 
expectations of individuals may change overtime (Carr \& Higginson). Therefore measuring QOL in a specific period of time will reflect how patients perceive their $\mathrm{QOL}$ at that time. For an adolescent, for example, factors that importantly dictate their QOL will change as they grow older, because their values and priorities are continually changing in response to changes in life (Carr \& Higginson). Moreover, research on patients from different countries indicate that $\mathrm{QOL}$ is independently determined by the level of an individual's depressive symptoms and mood (Mancuso, Peterson, \& Charlson, 2000; Moons et al., 2003; Ruo et al., 2003). Since depressive symptoms can reflect a temporary emotional state, $\mathrm{QOL}$ will change accordingly. Although the physical health of many dialysis people does not change a lot, other things in their life do such as employment, social and family relationships and physical activity. While QOL can change over time, it is unlikely to occur rapidly. Rather, QOL probably fluctuates little from one day to another. This research captured this concept at only one instant of time. This study used the SF-36 which asks participants to assess their QOL in the last four weeks. Due to time restraints and other logistic problems the researcher could not study the QOL of the selected participants over time. In addition, this study used the QOL Index to study the participant's perception and satisfaction of certain elements or dimensions of their life. The study did, however, capture several dimensions of time as possible factors that could impact on the QOL. These included time since commencing dialysis and time since last travelling abroad.

\subsubsection{Health-related quality of life (HRQOL)}

Most of the research studies measured satisfaction of QOL against a predetermined number of dimensions or areas of life (Bowling, 1995; Cummins, McCabe, Gullone, \& Romeo, 1994). This is true for both generic measures such as the SF-36 (Ware \& Sherbourne, 1992), and disease-specific measures such as the Diabetes QOL for Youth questionnaire (DCCT Research Group, 1988; Ingersoll \& Marrero, 1991) and the Audit of Diabetes Dependent QOL (Bradley et al., 1999). The dimensions incorporated in the health-related QOL tools are narrower and more specific than those employed by social scientists (Amarantos, Martinez \& Dwyer, 2001). The health-related QOL dimension is more bio-medically oriented, focusing upon physical and mental health dimensions that change with disease process and functional status, or treatment of these dimensions (Amarantos et al.).

Health is one of the fundamental components of QOL. Consequently, health-related QOL has been developed to capture the subjective life experiences that relate to 
health, disease, disability and impairment (Carr \& Higginson, 2001). Frequently, objective dimensions of disease and health are used as markers of QOL. This is in contrast to the assessment of QOL in healthy people, in whom the overall $\mathrm{QOL}$ is measured. However, even in patients with chronic and acute illnesses the difference between the overall QOL and health related QOL is obvious. Patients can distinguish between the parts of their life that are affected because of the disease and the parts that are affected by other reasons (Anderson \& Burckhardt, 1999; Macduff, 2000). By focusing on HRQOL, investigators may significantly overestimate the impact of healthrelated factors and under estimate the effect of nonmedical factors on QOL (Gill \& Feinstein, 1994). In this study, the QOL Index assessed both the health-related factors and the non health-related factors that may impact on the QOL and the SF-36 assessed the health-related issues that contribute to QOL. For instance, research on patients with severe medical conditions, such as Hodgkin's disease and other patients requiring peripheral blood stem cell transplants, found that only two-thirds of these patients pointed out health as important for their QOL (Frick, Borasio, Zehentner, Fischer, \& Bumeder, 2004; Wettergren, Bjorkholm, \& Langius-Eklof, 2005). Therefore, other factors are also important to their QOL.

\subsection{Culture and QOL}

Studying QOL in a multicultural population requires researchers to have a comprehensive understanding of what is meant by the term culture and how it links with QOL. Also, researchers need to understand how different cultures look at and how they value QOL. This section defines culture and explains the importance of understanding how culture impacts on QOL.

Although the concept of "culture" is used reqularly in health research, there is no universally accepted definition in health research (Johnson, 1996). Gudykunst and Kim (1992) found over 100 definitions of culture in their literature review. Three definitions which capture different aspects are listed next. The Center for Advanced Research on Language Acquisition at University of Minnesota defines culture as "the shared patterns of behaviors and interactions, cognitive constructs, and affective understanding that are learned through a process of socialization. These shared patterns of behaviors identify members of a culture group and distinguish those of another group" (Roshan Cultural Heritage Institute definition of culture, 2011, p.1). The Roshan Cultural Heritage Institute support cultural understanding through education and communication. This Institute refers to culture as "ways of life, including but not 
limited to: language, arts \& sciences, spirituality, social activity and interaction" (Roshan Cultural Heritage Institute definition of culture, p.1). The National Institute for Urban School Improvement (2011) defines culture as "a combination of thoughts, feelings, attitudes, beliefs, values, and behavior patterns that are shared by racial, ethnic, religious, or social groups of people". From these definitions one can conclude that culture is a shared system of beliefs and learned behaviors. The latter often being based on race, gender, ethnicity, religion, age, traditions, experiences, norms and expectations.

Understanding culture is critical for researchers and healthcare workers because one's cultural perspectives are present usually in interactions. Too often, people make assumptions about a person's beliefs or behaviours based on one factor, mainly race or ethnicity, when in reality, the cultural identities of people are a complex mix of all the cultural groups they belong to that influence their values, beliefs, and behaviors (The National Institute for Urban School Improvement, 2011). As researchers we need to keep in our mind that we cannot study culture without studying other variables that are linked with it such as gender, ethnicity, religion, age, norms and other variables that contributes to QOL. The National Institute for Urban School Improvement highlighted that cultural identity development is an ongoing process. On a daily basis we are exposed to more and different sets of beliefs and values, and we may chose to adopt ones that were not part of our original upbringing. So, cultural identity is built within the individual, but continually influenced by the interactions among and between people in the society. Often, culture is considered to be the foods, music, clothing, and celebrations a group of people share yet it is more than just these visible things. Culture refers not only to what we are born into (racial or ethnic groups), but also what we choose to belong to, such as a religious or social group (The National Institute for Urban School Improvement).

Normative data are important for determining at a country or groups level. While the average normative values can be used to compare whether a group or an individual scores below or above the average for their country, age or sex. Published norms for the SF-36 now exist for some countries such as the United Kingdom (Jenkinson, Stewart-Brown, Petersen, \& Paice, 1999), Turkey (Altintepe et al., 2006), Italy (Apolone \& Mosconi, 1998), Taiwan (Tseng, Lu, \& Gandek, 2003), Canada (Hopman et al., 2000) and northen part of Jordan (Khader, Hourani, \& Al-Akour, 2011). Representative samples from either a control group or a general population from a country are 
normally used to establish the normative values for the SF-36 tool (Yarlas et al., 2011). No information is available on the normative values of the QOL Index tool.

The Turkish (Altintepe et al., 2006) and the Jordanian (Khader, Hourani, \& Al-Akour, 2011) studies were done on Muslim populations from the Middle East. The aims of these studies were different; the Turkish study aimed to generate population norms for the Turkish version of SF-36 and to ascertain the association of SF-36 domains with demographic and socioeconomic variables and self-reported ill-health in a general urban population. The Jordanian study was specifically designed to assess the psychometric properties of version one of the SF-36 and to establish population norms among the general population of north Jordan. The method of data collection methods in the above mentioned studies were identical. Both studies used face-to-face interviews with the participants.

The Jordanian study measured the normative values of six districts in north part of Jordan. So, these normative values represent the north part of Jordan only (Khader, Hourani, \& Al-Akour, 2011), therefore it is not representing all Jordanian population. The Turkish QOL scores were from a study aimed to determine prevalence of psychotic disorders in the adult population. The sample was only from Izmir, which is the third largest city in Turkey. This city is economically well developed compared with other urban cities in Turkey. So the sample might not be representative to all population in Turkey. In summary, the samples in the Jordanian and the Turkish studies were regionally representative.

Application of the norm-based scoring methods simplifies interpretation, allowing a reader to compare findings between scales as well as between studies (Bowling, Bond, Jenkinson, \& Lamping, 1999). Unfortunately, norms do not yet exist for the UAE. Establishing normative values of the SF-36 will enable researchers to compare data from the UAE study with those from other countries.

\subsection{QOL in Dialysis Patients}

\subsubsection{Background}

Dialysis treatment causes a major life change for patients. Dunn et al. 1994 described three stages that most dialysis patients go through. The first stage is called the 'honeymoon period' which is marked by improved physical well-being after the start of 
dialysis. The second stage is 'the disenchantment or discouragement period', is characterised by the onset of sadness, denial, hopelessness and helplessness in which a wide range of clinical disorders such as fatigue, physical decline, peritonitis, sexual issues and cognitive impairment often occur (Klang \& Clyne, 1997; Lok, 1996; Mitchell \& Goosby, 1996). The third stage, 'long-term adaptation', occurs when the patient finally reaches some level of acceptance of their disease and its limitations. Some patients never reach this stage of adaptation (Dunn et al., 1994).

The literature review reported statistically significant differences in QOL between the healthy general population and dialysis patients (Cleary \& Drennan, 2005; Kutner et al., 2001; Vasilieva, 2006). Patients with kidney failure experience long term debilitating illness that has major limitations on their physical, psychosocial abilities that tamper with their ability to live normal life (Valderrabano, et al., 2001), while people from the general population are considered relatively healthy but may experience other types of stressors in their life mainly resulting from other aspects rather than being sick. Therefore, it will be a great value to compare the QOL between dialysis patients and a sample from the community in UAE.

\subsection{Socio-demographic factors clinical variables contributing to QOL in dialysis patients}

Most studies looked at certain aspects of socio-demographic factors and QOL. In this study, the researcher examined a wide range of socio-demographic factors such as gender, ethnicity, religion, social and family support, marital status, employment, education level and age. Also the researcher studied various clinical variables such as presence of other chronic illnesses, knowing the cause of kidney failure, length of time on dialysis, and laboratory test results values (haemoglobin, serum creatinine, serum albumin, and dialysis adequacy).

\subsubsection{Socio-demographic factors contributing to QOL in dialysis patients}

\subsubsection{Gender}

The influence of gender on QOL was regularly examined in the dialysis literature. In the main this examination only looked at whether there were differences in male and female scores on the QOL measures. The review of the literature revealed contradictory results. The study by Acaray and Pinar (2005) of the QOL of 100 Turkish hemodialysis patients and another by Kalantar-Zadeh, Kopple, Block and Humphreys 
(2001) of 65 American hemodialysis patients both found that gender was not significantly different in determining QOL using the SF-36. In contrast, Mingardi et al.'s (1999) study of 304 Italian dialysis patients using the SF-36 reported that the QOL of the individuals varied according to gender. The physical dimension of QOL was higher in males.

\subsubsection{Ethnicity}

Several studies have examined the effect of ethnicity on QOL. Studies done with African American hemodialysis patients reported better health status and QOL compared with Caucasians (Kutner, Brogan, Fielding, \& Hall, 2000; Kutner \& Devins, 1998). Kutner et al. consider that African American patients possess "hardiness" as part of their biological make up that is not captured by the routinely collected clinical status variables and QOL tools. Furthermore, African Americans might have greater perception of social supports compared with Caucasians patients (Kutner et al., 1998). This could promote the sense of well-being for African American patients compared with Caucasians patients (Kutner et al., 2000).

The survey by Hicks et al. (2004) of 1392 dialysis patients from four different regions in the United States of America (USA) using the Kidney Disease Quality of Life (KDQOL) tool had similar results. Dialysis patients who had African American ethnicity were physically active and had more family and community influences on their health status and QOL. Potential explanations for the observed ethnicity differences in dialysis patients' reported health status and QOL include biologic differences, socio-cultural differences and different experiences of access to healthcare before dialysis started.

Bakewell, Higgins and Edmunds (2001) compared the influence of ethnicity on QOL of 60 Indo-Asian patients (20 on hemodialysis, 20 on peritoneal dialysis, and 20 had kidney transplant) with 60 age-matched white Europeans matched for sex, diabetes and time on renal therapy using the Kidney Disease and QOL questionnaire (KDQOL-SF) in United Kingdom. Bakewell and colleagues found that Indo- Asians had lower scores of perceived QOL compared with white Europeans with Kidney failure. Patients from different ethnicities perceive their QOL differently. They demonstrated that Indo-Asians perceive their kidney disease as a social burden that influenced their ability to work as well as being a burden on their family members. However, the IndoAsian sample in Bakewell and colleagues study were originally from the Indian subcontinent region and most patients do not all share the same language, religion, 
social class or cultural factors such as family values. This demonstrates that it is difficult to measure ethnicity because of the variability in the cultural and value system in people from the same ethnic groups.

\subsubsection{Religion}

Patel et al. (2002) studied the importance of faith (spirituality), attendance at religious services and influence of adhering to religion with 53 hemodialysis patients on a range of measures. Measures included the Beck Depression Inventory, Illness Effects Questionnaire, Multidimensional Scale of Perceived Social Support, McGill QOL and Karnofsky tools. Patel et al. found a relationship between high scores on spiritual beliefs scale and global QOL measures, satisfaction with life and perception of depression. Greater perception of spirituality and religiosity has been linked to increased perception of social support and QOL and less negative perception of illness effects and depression (Patel et al.; Spinale et al., 2008). Kidney failure accompanied with many co-morbidities and other long term complications of dialysis. Religious beliefs impact on dialysis patients' ability to cope (Ko et al., 2007).

\subsubsection{Social and family support}

The effect of social and family support of dialysis patients on QOL is significant in the literature. A review of family support among African American patients with kidney failure found to be varied from over-involvement to no support and social isolation from their family. Families as well as patients with chronic illness were at risk of high levels of stress (Holder, 1997). Family members experience stress for long periods of time and need to constantly adapt as the disease progresses (Newby, 1996). Family and marital relationships that have evolved prior to the patient becoming ill may be altered by the presence and attached responsibility to caring for a person on dialysis. It is known that the family's adaption is influenced by factors such as the quality of family relationships (Beanlands et al., 2005), cultural values and beliefs (Lindqvist et al., 2000).

Elal and Krespi (1999) reported that social support is associated with improved psychological adjustment of dialysis patients and depressed dialysis patients reported less social contact with others and perceived had little social support (Elal \& Krespi, 1999). Sarason, Sarason and Pierce (1990) suggested that perceived support is a part of an individual's personality. Depending on the nature of people's personality, the satisfaction they feel from the social support they received might be even more critical 
than the perceived availability of social support, in association with their psychological well-being. Thus, the impact of satisfaction from the received social support may vary depending on people's personality characteristics (Gencoz \& Astan, 2006).

\subsubsection{Marital status}

Kao et al. (2009) evaluated the associations between economic, social, psychological factors, and health-related QOL of 861 hemodialysis patients from Taiwan using the SF-36. Kao and colleagues found that marital status was not associated with healthrelated QOL. Their findings are supported by a study on Hong Kong dialysis patients using the Chinese Dialysis QOL Scale, in which it was found that marital status had no impact on QOL (WI, 2001). In contrast, Steele et al. (1996) found that more than half of patients with kidney failure had evidence of marital disruption. The development of kidney failure may place strain on usual marital roles and might change patients' ability to work, forcing a shift in individuals' roles within the family. The potential damaging impact of chronic kidney failure on a patient's psychosocial function and the marital strain experienced by patients on dialysis is well documented (Shidler, Peterson, \& Kimmel, 1998). Acaray and Pinar (2005) studied 100 Turkish dialysis patients using the SF-36 and reported that the overall QOL score was significantly higher in single patients except for the mental health and role emotional subscales when compared with that of married and widowed or divorced dialysis patients. A possible explanation for this result was that the single participants had less responsibilities compared with married dialysis patients. Therefore, this reduces the psychosocial burdens of the disease. Furthermore, their families may shoulder the economic burden and other responsibilities that normally impact on their perception of the quality of their life.

Spouses can become caregivers and may develop depression and/or verbal abuse. They may have to change work patterns, forcing a shift in their role within the family. In addition, the spouse may be the object of the patient's negative emotions (Palmer, 2003). Decreased marital satisfaction and disturbances in family dynamics have been associated with poorer health outcomes and can affect patients' perception of social support and depressive affect (Cukor, Cohen, Peterson, \& Kimmel, 2007). CoelhoMarques, Wagner, Poli de Figueiredo and d'Avila (2006) used a cross-sectional controlled survey design to assess demographic, marital, sexual activities on QOL in 86 healthy women aged 18 years and over (Group 1), and in 38 female patients on dialysis for at least two months (Group 2). QOL was studied using the World Health Organization QOL tool. Coelho-Marques et al. found that the overall QOL was lower in 
Group 2, especially on physical and environment domains and female patients undergoing dialysis had lower $\mathrm{QOL}$ and were also significantly more sexually dysfunctional than the healthy women.

Dialysis patients frequently experience a marked deterioration in their self image and considerable difficulties in their sexual drive and sexual performance (Zarifian, 1994). Sexual dysfunction can change a marriage and impact negatively on marital relationships as well as QOL (Palmer, 2003). Psychosocial issues, such as changes in body image, can contribute to the sexual problems experienced by dialysis patients (Camsari et al., 1999). Patients on hemodialysis who have an intra-jugular central line, may suffer body-image shock when they realise they have a line protruding from their necks. What might be viewed by nurses and doctors as good working access may be perceived by the patient as disfigurement (Levy et al., 2004; Thomas, 2002). Physical abnormalities and scars are common in patients with kidney failure due to the frequent insertion of needles required for dialysis treatment (Hutchful, 1980). The dialysis vascular access alters the body image, which may affect the level of attractiveness to partners and impact negatively on the marital relationship as well as QOL.

\subsubsection{Employment}

The long term medical complications resulting from kidney failure lead to a decline in physical functioning. Diminished working capacity and energy levels often lead to inability to continuo full-time employment (Ferrans \& Powers, 1992). The stresses resulted from being financially dependent along with difficulties in coping with family responsibilities, and social lives leads to mood swings and unfulfilled hopes (Ferrans \& Powers). Patients with chronic illnesses encounter stressful situations in their work environment. Studies have found that factors such as dialysis duration (van Manen et al., 2001); educational level, physical functioning ability and co-morbidities were associated with the work status of patients undergoing dialysis treatment (Molsted, Aadahl, Schou, \& Eidemak, 2004). Furthermore, Takaki et al. (2005) assessed the effects of age, sex, time on hemodialysis, level of education employment and income, and coping mechanisms on depression and anxiety in 416 patients on hemodialysis. They found that employment promoted coping and minimised depression in dialysis patients. In contrast Kao and colleagues (2009) found that employment was not associated with health-related QOL in a study done on 861 dialysis patients from Taiwan. 
Patients with kidney failure experience several physical limitations. Studies on patients with kidney failure shows that these patients suffer from disease-specific symptoms (Buargub, Nabulsi, \& Shafeh, 2006), diminished physical working capacity (Sterky \& Stegmayr, 2005), inability to pursue full time employment (Neri et al., 2005) and difficulties in coping with family responsibilities and social lives (Mok \& Tam, 2001). van Manen et al. (2001) and Krediet (2001) and members of the Netherlands Cooperative Study on Adequacy of Dialysis have found that within one year the proportion of employed patients decreased from $31 \%$ to $25 \%$ in hemodialysis patients, and from $48 \%$ to $40 \%$ in peritoneal dialysis patients. Loss of work is an important issue in both pre-dialysis and dialysis patients. Furthermore, Kutner, Progan and Fielding (1991) interviewed 283 dialysis patients, age 18-59 years, about their current work status and their ability to work if currently not employed. This study revealed that only $11 \%$ of the participants were currently employed and one third of the non-employed said they were able to work but had been unsuccessful in their efforts to find employment. Age, race, educational level, health status and physical ability to perform job tasks, recent work experience, and interest in working were related to the reported ability to work.

\subsubsection{Education level}

Several studies have reported a relationship between educational level and QOL. Each of the studies used a different QOL tool. Pakpour et al. (2010) studied 250 Iranian haemodialysis patients using the Persian version of the SF-36 and found that patients with lower level of education have poor QOL scores. Moreover, Acaray and Pinar (2005) studied the differences in the educational levels of four groups of dialysis patients (no formal education, primary school, secondary high school and university graduates). They reported that the overall total scores of the SF-36 increased as educational status increased. Mozes, Shabtai and Zucker (1997) studied 680 patients receiving dialysis therapy using the QOL Index and Suet-Ching (2001) studied QOL in 164 Hong Kong dialysis patients using the Chinese Dialysis QOL Scale. Both studies linked high QOL scores with higher education level. Additionally, Moreno et al. (1996) reported similar findings in a randomly selected sample $(n=1013)$ of stable Spanish dialysis patients using the Karnofsky Scale (KS) and the Sickness Impact Profile (SIP) tools. In contrast a study done by Kao and colleagues (2009) found that educational level was not associated with health-related QOL. Nevertheless higher levels of education appear to positively affect and promote healthy. Educated dialysis patients could take some responsibility of their own health and thus would learn and employ 
strategies to cope with their disease and its symptoms, leading to an enhanced QOL (Mingardi et al., 1999; Moreno et al.; Neto et al., 2000).

\subsubsection{Age}

The effect of age on QOL is controversial in the literature. Age accompanied with comorbidity may have a negative impact on QOL (Jager et al., 2003; Loos, Briancon, Frimat, Hanesse, \& Kessler, 2003; Mapes et al., 2004). QOL therefore depends mainly on the severity of co-morbid conditions and the physical decline over time. Hsieh et al. (2010) evaluated the physical capacity (6-minute walk test, grip strength, pinch strength and chair-rising time), maximal cardiovascular fitness test and functional performance for 27 ambulatory hemodialysis patients living in the community for 16 months in Taiwan. They found that the physical capacity declined over the 16 months.

Kao and colleagues (2009) evaluation of the associations between age and healthrelated QOL of hemodialysis patients from Taiwan using the SF-36 found that age was significantly inversely associated with physical functioning, role physical, vitality and social function, but not the mental health subscales of the SF-36. When older dialysis patients were compared with younger ones ( $<60$ years) in terms of dialysis treatment, older patients had less stress and a better perception of QOL (Grapsa \& Oreopoulos, 1996). Loos et al. assessed the effect of kidney failure on the QOL of 169 older dialysis patients from 13 dialysis units in France using the SF-36. They found that elderly patients who had planned pre-dialysis education and preparation had better QOL scores compared with elderly dialysis patients who did not have any pre-dialysis preparations. As the dialysis population ages, the prevalence of chronic disease overall is also likely to increase (Berthoux et al., 1998). Patients who have fewer chronic health problems generally report better QOL than those with more co-morbidity, particularly in the physical and psychological dimensions (Testa \& Simonson, 1996). Older age therefore is known determinant of QOL of kidney failure patients.

\subsubsection{Clinical variables contributing to QOL in dialysis patient}

\subsubsection{Chronic health problems}

Co-morbid medical conditions are common in patients on dialysis. They are considered as important contributing factors to clinical outcomes and QOL. Associated diseases, especially diabetes mellitus, are strongly related to the worst QOL scores in kidney failure patients on dialysis (Bakewell, Higgins, \& Edmunds, 2002; Kalantar-Zadeh et al., 
2001). In a representative assessment, the Dialysis Outcomes and Practice Patterns Study documented the most common disorders including cardiovascular diseases, hypertension and diabetes in 3,856 patients who were new to dialysis. Cardiovascular disease remains the major mortality risk in dialysis patients, accounting for more than $50 \%$ of deaths among these patients (Foley, Parfrey, \& Sarnak, 1998). Hyperphosphatemia and elevated calcium phosphorus product are associated with cardiovascular calcification, including the aorta, carotid and coronary arteries and cardiac valves, as well as the myocardial muscle (Qunibi, 2004). Hyperparathyroidism is one reason why patients may have impaired response to recombinant human erythropoietin in patients with kidney failure (Drueke \& Eckardt, 2002). The complications of elevated Para-thyroid hormone are expected to decrease survival and QOL in dialysis patients (Drueke \& Eckardt).

Chronic kidney failure impacts on other aspects of life. The life of patients treated with dialysis is characterised by many losses and restrictions. Even the selection of food and beverages, practice for a sport or accepting an exerting job, may be a problem. People with chronic illnesses may forget what a 'normal' life is like and develop changing views of normalcy developing new ways of doing and new ways of coping (Fayer \& Sprangers, 2002). Restriction of fluid or food, pain, itching, discomfort, limitation of physical activities, fatigue, weakness, cost of care, feelings of inadequacy and negative moods were identified as the major physiological and psychosocial stressors (Lok, 1996; Mok \& Tam, 2001; Welch \& Austin, 2001). The field of QOL is broad and includes physical health psychological, social, educational and occupational well-being. Kidney failure and its treatments usually disturbe people lives. Stapelton (2000) categorises stressors confronting patients with kidney failure as physiological, psychological, role-disturbance and life-change stressors. Having a chronic condition such as kidney failure is likely to have an impact on patients' everyday life. Dialysis patients are usually asked to follow medical advice that changes their normal routine. In addition to dialysis three times a week for the rest of their life, patients have to take medications and follow a strict diet and limit their fluid intake (Betts \& Crotty, 1998). Dialysis patients are also subjected to multiple physical and psychosocial stressors that may change their life style (Welch \& Austin).

\subsubsection{Knowing the cause of kidney failure}

Educational and psychological interventions can produce important benefits for dialysis patients. Pre-dialysis psycho-educational interventions, for example, can enhance 
illness-related knowledge (Mehrotra, Marsh, Vonesh, Peters, \& Nissenson, 2005) and promote QOL (Fukuhara et al., 2006). Several studies have demonstrated that early referral to nephrologists decreases morbidity, mortality and healthcare costs (McLaughlin, Manns, Culleton, Donaldson, \& Taub, 2001); improves long-term survival (Jungers et al., 2001); reduces the need for urgent dialysis (Schmidt, Domico, Sorkin, \& Hobbs, 1998); and improves health-related QOL in dialysis patients (Korevaar et al., 2002). Klang, Bjorvell, Berglund, Sundstedt and Clyne (1998) evaluated the effects of a pre-dialysis patient education programme on functioning and well-being in 28 dialysis patients three to nine months after starting dialysis. They found that patients who received dialysis education had higher mood scores, lower mobility problems, lower functional disabilities and a lower level of anxiety. Furthermore, Acaray and Pinar (2005) found that patients who were educated on the disease and on hemodialysis had significantly higher scores in all dimensions of the SF-36, except role-physical functioning, body pain and mental health dimensions than patients who were not educated. Pakpour and colleagues (2010) found that patients who had low knowledge about the disease process had poor QOL scores.

Few studies linked understanding the disease process and ability to cope with chronic illnesses. Tsay, Lee and Lee (2005) studied the effectiveness of an Adaptation Training Programme (ATP) to help patients with kidney failure to cope with illness-related stresses and improve QOL. Tsay et al. used a randomised controlled trial with a convenience sample of 57 eligible dialysis patients who were assigned to experimental (ATP plus usual care) or control (usual care) groups. After eight weeks, patients were asked to complete the Haemodialysis Stressor Scale, Beck Depression Inventory and SF-36. Tasy et al. concluded that the major stressors for these patients were limitations on when and where they could work, type of transport they could use, and length of dialysis treatment.

\subsubsection{Length of time on dialysis}

The length of time on dialysis could lead to the extension of suffering from the consequences of kidney failure. Dialysis patients not only face treatment-related stressors but have to deal with changes in their life, self-confidence and family roles (Lev \& Owen, 1998). Bohlke et al. (2008) used a cross-sectional design to study the predictors of QOL in 140 patients undergoing dialysis (94 on hemodialysis and 46 on peritoneal dialysis) in three southern Brazilian dialysis facilities using the SF-36. They found that patients who had been on dialysis for short lengths of time had higher QOL 
scores compared to patients who have been on dialysis for longer period of time. Moreover, co-morbidity and length of time on dialysis were the main predictors of physical QOL, whereas socioeconomic issues especially determined mental QOL. Furthermore, Mittal, Ahern, Flaster, Maesaka and Fishbane (2001) evaluated the QOL of 134 American haemodialysis patients using the SF-36 three-monthly over two years. They found that the number of months on haemodialysis had a significant inverse relationship with the changes in physical function, body pain and general health vitality subscales scores of the SF-36. Pakpour et al. (2010) found that the longer time on dialysis correlated with poor SF-36 scores.

\subsubsection{Haemoglobin and anaemia}

Anaemia has a negative effect on QOL. Partial correction of anaemia to maintain haemoglobin $(\mathrm{Hb})$ levels in the target range of 11 to $12 \mathrm{~g} / \mathrm{dL}$ recommended by the National Kidney Foundation's Kidney Disease Outcomes Quality Initiative (NKF$\mathrm{K} / \mathrm{DOQI}(\mathrm{TM})$ ) typically leads to significant improvements in both physical and mental health-related QOL in dialysis patients (National Kidney Foundation, 2006). The correction of anaemia is usually done by administering Erythropoietin (EPO). EPO is a naturally occurring hormone, produced by the kidneys, which stimulates the bone marrow to produce red blood cells (Valderrabano, 1996). Failed kidneys produce less EPO resulting in patients with kidney failure suffering from anaemia (Speigel, 2006). Anaemia negatively influences patients energy and activity levels, sleep and eating behaviour, general health status, sex life, and can cause muscle weakness, leg cramps and shortness of breath (Breiterman-White, 2005), therefore reducing the QOL overall.

Treating anaemia with EPO reduces the morbidity and mortality, hospitalisation, and left ventricular hypertrophy, and improves QOL (Madore et al., 1997; Speigel, 2006). The severity of anaemia and success of its treatment are also affected by other factors such as iron stores (Mircescu, Garneata, Capusa, \& Ursea, 2006), presence of inflammation, infection, malnutrition (Kalantar-Zadeh et al., 2003), and hyperparathyroidism (Drueke \& Eckardt, 2002). Studying QOL in dialysis patients without paying attention to anaemia severity, presence of inflammation, malnutrition and hyperparathyroidism may influence the overall QOL rating.

\subsubsection{Dialysis adequacy}

Traditionally, hemodialysis dose has been quantified referring to the kinetics of urea. For this purpose, different methods are available. Frequently used is the index $\mathrm{Kt} / \mathrm{V}_{\text {urea }}$, 
the product of urea clearance $(K)$ times the length of the treatment time $(t)$, in relation to the urea distribution volume ( $V$ ) of the patient. In 2006, updated K-DOQI guidelines advocated the use of the single-pool Kt/ $\mathrm{V}_{\text {urea }}(\mathrm{spKt} / \mathrm{V})$, derived from the single-pool variable volume urea kinetic model. The K-DOQI guidelines recommended a dialysis adequacy expressed in a KT/V formula of greater than or equal to 1.3 (NKF-KDOQI clinical practice guidelines, 2006). The impact of dialysis adequacy on the QOL for dialysis patients is still debatable in the literature.

Cleary and Drennan (2005) studied the dialysis adequacy and QOL of 97 patients undergoing haemodialysis treatment at a hospital in the Republic of Ireland using the SF-36. They identified limitations in a number of areas including vitality, physical functioning and physical role limitations in addition to significant differences in mental health scores between patients who were well dialysed and those less well dialysed. Cleary and Drennan also reported significantly lower physical functioning when compared with a general population group. Furthermore, Manns et al. (2003) used a cross-sectional survey design to study the dialysis adequacy on 128 patients who had been on hemodialysis for more than six months. Average dialysis adequacy $\mathrm{Kt} / \mathrm{V}$ levels (for the three months preceding health-related QOL assessment) were determined. QOL was assessed with the SF-36 and the EuroQOL EQ-5D. Manns et al. concluded that patients with average $\mathrm{Kt} / \mathrm{V}$ levels greater than or equal to $1.3 \mathrm{had}$ higher SF-36 scores and higher EuroQOL EQ-5D scores.

In contrast, the study by Morton et al. (1996) on the impact of dialysis adequacy on QOL of 55 hemodialysis and 60 peritoneal dialysis patients using the SF-36 tool found no significant association between $\mathrm{Kt} / \mathrm{V}$ and any of the dimensions (physical functioning; role limitations (physical); role limitations (emotional); social functioning; emotional well being; pain; energy; and general health perceptions) of QOL. These findings were supported by Eknoyan, Beck, Cheung and Daugirdas (2002) who noted that patients undergoing hemodialysis thrice weekly appear had no major benefit from a higher dialysis dose than that recommended by current USA guidelines or from the use of a high-flux membrane.

Twardowski (2004) showed that short, three times weekly hemodialysis was inadequate and did not improve patient outcome. However, daily hemodialysis with a minimum of six sessions per week is regarded to improve dialysis patient outcome. Daily dialysis and improved better fluid management results in patient's experiencing 
“legs cramping episodes during dialysis, fewer headaches, less hypotension, fewer episodes of dizziness, decreased fluid restriction, decreased interdialytic weight gain, fewer episodes of shortness of breath and a reduction in the sensation of easily feeling cold" (Heidenheim, Muirhead, Moist, \& Lindsay, 2003, p. 462). Furthermore, in a multicentre, prospective, randomized, parallel-group trial conducted by the Frequent Hemodialysis Network (FHN) Trial Group, Chertow, et al. (2010) compared frequent (six times per week) hemodialysis, with conventional (three times per week) in-center hemodialysis on a total of 378 patients from 54 community-based hemodialysis facilities in North America. The trial reported that frequent daily dialysis improves optimal blood pressure control, regression of left ventricular hypertrophy, optimal hyperphosphataemia control, indices and improvement in QOL.

\subsubsection{Albumin}

Albumin level has been linked to morbidity and mortality in dialysis patients (Mapes et al., 2004). Knowing which biomarkers influence QOL may therefore provide insight into how best to manage ESRD. Markers of Malnutrition-Inflammation Complex Syndrome (MICS) are reported to predict mortality and hospitalisation in haemodialysis patients. However, it is not clear which marker is more sensitive and predictive of outcome. Kalantar-Zadeh, Kopple, Humphreys and Block (2004) examined the utility of 10 markers of MICS as (predictors of mortality and hospitalisation, malnutritioninflammation score, a subjective global assessment score, and serum levels of Creactive protein, interleukin-6, tumour necrosis factor-alpha, albumin, pre-albumin, total iron binding capacity, creatinine, total cholesterol and normalised protein nitrogen appearance). The study by Kalantar-Zadeh et al. of a cohort of 378 dialysis patients (average 55 years; 53\% men; 47\% Hispanics, 30\% African-Americans; 55\% diabetic patients), who were randomly selected from dialysis facilities in Los Angeles, found that C-reactive protein, malnutrition-inflammation score and Charlson co-morbidity index were the only consistent predictors of mortality and hospitalisation, and their outcome predictabilities were superior to serum albumin. In contrast, other studies suggested that anaemia control and normal albumin levels were associated with improved survival and QOL (Lopes et al., 2007; Sanaka, 2003).

\subsection{Literature gaps}

Little is known about the differences in QOL between dialysis patients and health population from different cultures. Most of the data found about the QOL in the general population were in conjunction with a comparison of the QOL of patients with chronic 
illnesses. Casetta et al. (2009) compared the gender differences in health-related QOL in multiple sclerosis patients and a sample from the community in Italy. Schweikert et al. (2009) compared the QOL of myocardial infarction patients with a sample from the community. Vasilieva (2006) compared the QOL in chronic hemodialysis patients with 2114 people from the community in Russia. Moreover, Molsted et al. (2004) assessed the health-related quality of life in dialysis patients with 4080 people from the community in Denmark.

Several QOL tools have been used to study QOL. However, not all studies looked at the same number of variables and each tool has different subscales. Generally, the rationale for tool selection is not well stated. Researchers sometimes replicated other studies and used the same tool; some of them used the most published tools. To what extent QOL tools measure the same components of QOL have not been extensively examined.

The literature review revealed that all the QOL tools had been tested for reliability and validity but the cultural relevancy of those tools not been examined. As this is the first study in UAE, the researcher examined the QOL of dialysis patients and a sample from the community using two tools (the SF-36 and QOL Index). In addition to that this study examined the selected tools for cultural relevancy simultaneously to ensure that these tools were culturally relevant for the participants who had an Islamic and Arab cultural background. Establishing a clear understanding of the sensitivity of tools to the traditions and culture of people completing them will help in giving a true reflection and accurate measures of QOL.

Few research studies have been found in the literature that compares two QOL tools on the same sample of dialysis patients (Neto et al., 2000). Neto et al. used the SF-36 to study the QOL of 80 dialysis patients, and used a second tool KDQOL questionnaire on 22 randomly selected patients from the total sample of 80 participants. Neto et al. found that SF-36 dimensions correlated significantly with those of the KDQOL questionnaire. Huang, Wu and Frangakis (2006) compared the psychometric properties and factor structures of the SF-36 and World Health Organization Quality Of Life - Breif (WHOQOL-BREF) tools on Taiwanese population. Data were collected from a national representative sample of 11,440 people. They concluded that, the SF-36 and WHOQOL-BREF appeared to measure different constructs: the SF-36 measures health-related QOL, while the WHOQOL-BREF measures global QOL. 
The literature review revealed that dialysis patients' QOL are highly affected when they are exposed to major physical, psychosocial and financial stressors. However, the above-mentioned determinants may not all be applicable to dialysis patients in UAE because they have not been examined in this population group. This is the first study to examine QOL of dialysis patients in this country. There is an increased interest in studying QOL worldwide. However, there is inconsistency or disagreement on the definition of QOL. Given what is known about the subjective and objective nature of QOL it was necessary to study the subjective and objective nature of QOL in dialysis patients and community in UAE. As the information about the indicators and determinants of QOL is mainly from research carried out in western countries, it will be of great value to study the indicators and the determinant of QOL in dialysis patients and a sample of healthy population in UAE, keeping in mind the importance of the individual's subjective appraisal of their state of health as well as QOL. Furthermore, this study was designed to establish what is important in respect of QOL for dialysis patients and a sample from the community living in UAE using two QOL tools. studying a sample from the community and one with known kidney failure will help in creating a sound comparison between the QOL of both samples in terms of the impacts of physical, psychosocial, cultural, gender, ethnicity and presence of chronic illness on QOL.

The experience of different ethnicities and religious beliefs of people living on dialysis might be significantly different. Researchers have examined the QOL of African American, Chinese, Japanese, Indo-Asian and white Europeans. No information was found in the literature with regards to Islam as a religion and Arab as an ethnicity or race and $\mathrm{QOL}$ in dialysis patients. However, this research studied the impact of ethnicity on the QOL of UAE culture mix population.

\subsection{Conclusion}

It was highlighted that some definitions of QOL focus on physical and psychological function, whilst others acknowledge that QOL also relates to the individual in the context of their culture, beliefs, values, goals and expectations. This chapter has evaluated and critiqued the appropriateness of different conceptualisations of QOL. The terms health status, functional status, and QOL should not be used interchangeably and healthy life does not equate to a high QOL. A conceptualisation of QOL comprising both objective and subjective dimensions dominates research on 
QOL. There is a poor distinction between indicators and determinants of $Q O L$ in the literature. When assessing QOL, researchers should examine the negative and positive elements that contribute to it. People perceive and cope with the negative components of their life may vary among ethnic or cultural groups. Life is not static and QOL cannot be considered to be a static as well. Individuals may appraise their QOL differently over time due to ever-changing life events, illness progress, coping abilities or cultural circumstances. When people grow older, their values and priorities change in response to life stages, psychological condition and circumstances. Overall QOL is influenced by numerous factors in addition to health; thus a comprehensive instrument must not be limited strictly to health-related items. QOL is clearly not solely dependent on physical function, nor can it be viewed irrespective of the patient's physical, psychological, social and cultural circumstances.

A review of the literature on the effect of gender on the QOL of hemodialysis patients revealed contradictory findings. Some studies claimed no effect of gender on the QOL, while others reported that the physical dimension of QOL was higher in males. The literature suggested that the experience of different ethnicities and religious beliefs of people living on dialysis might be significantly different. No studies were found in the literature, with regards to Islam as a religion and Arab as an ethnicity or race and QOL in dialysis patients. Social and family support plays an important part in the QOL of dialysis patients. Social support is associated with improved psychological adjustment of dialysis patients. The potential damaging impact of kidney failure on the marital relationship and QOL is well documented. Deterioration and difficulties in sexual drive and sexual performance place a lot of strains on the marital relationship. Changes in body image can impact negatively on their perception of QOL. The limitation in physical activities has been linked to inability to pursue full-time employment. Unemployment makes them financially dependent on others. Educational level has been linked to better QOL. Higher levels of education positively affect and promote health behaviours. Kidney failure combined with other chronic illnesses such as diabetes has major impact on the QOL. Knowing the cause of kidney failure and understanding the treatment plans had a positive impact on QOL and may lead to better QOL. Older dialysis patients often have co-morbid conditions that may affect QOL. The length of time on dialysis could lead to the extension of suffering from the consequences of kidney failure. Anaemia is a symptom of chronic kidney failure and exerts markedly adverse effects on the QOL of dialysis patients. Anaemia control and good nutritional status reflected in normal albumin levels were associated with improved survival and QOL. 
The impact of dialysis adequacy on the QOL for dialysis patients is still debatable in the literature.

The literature review and the selection of variables used in this study have informed the research design. Given the multi-dimensional nature of $\mathrm{QOL}$ and the inconsistency in what constitutes the important elements of QOL, this research investigated the nature of $\mathrm{QOL}$ in both samples. In an attempt to capture key dimensions of QOL, two QOL tools were used. In addition, three open-ended questions on what participants personally valued most in life, what things people living in UAE valued most in life, and what things were most important to participants in maintaining or improving their QOL were asked. These questions were included to capture any elements that might not be captured by the two QOL tools used in this study. 


\section{Chapter 3 Quality of life tools and the conceptual framework}

This chapter is in two parts. It commences with a brief description of the relationship between culture and QOL. Then it gives an overview of QOL tools and a description and critique of the two QOL tools. It then presents the conceptual framework that was developed to inform data collection and analysis for this research. The conceptual framework provides a structure to investigate the contributing factors to $\mathrm{QOL}$ and explores how these factors influence outcomes in dialysis patients. The conceptual framework describes the interaction of different variables on the individual's perceptions of their QOL such as culture and society, religion, physical health, family and social support, economic resources, and life events.

\subsection{Culture and QOL tools}

Most QOL instruments have been developed in western cultures and translated to other languages such as the Arabic language. This is an issue that needs to be critically examined. Certainly, translating and using an existing standardised instrument has the advantage of being cost effective, quick, and allows comparison of studies. A potential danger comes from close adherence to the original language version, where the translations were done to reflect the meaning in the original tool culture and language. Sen and Man (1986) argued that differences in cultural norms, belief systems and values across the world could be important reasons for the inadequacy of QOL research in countries having different languages from where the $\mathrm{QOL}$ tools originated. Kapur (1992) pointed out that during the process of maintaining similarity of interpretation and response; things may need to be expressed quite differently in two different cultures. This indicates that an instrument developed in one culture may be unsuitable to be used by another culture.

Cultural differences can threaten construct validity because the items or questions used may mean different things in different cultures. Aiken (1996) argued that clear operational definitions of the indicators constructs such as QOL are needed so that amongst cultures the same phenomina is measured.

It is important to develop QOL tools that are culturally appropriate, with well established reliability and validity in the target population (Ramirez, Ford, Stewart, \& Teresi, 2005). This requires establishing the cultural relevance, validity, and reliability of the tools in the target population. Researchers translating QOL tools from English to Arabic 
needed to follow well established guidelines for translation and cultural adaptation of QOL tools for Arabic populations. Using culturally appropriate tools to measure QOL in Arabic populations will provide an accurate data on QOL and therefore help nurses deliver individualised nursing care to the dialysis population in the UAE.

\subsubsection{Why assessing cultural relevancy of QOL tools?}

Most QOL tools are valid and reliable for measuring QOL in the mainstream English speaking population and contain content that is relevant to the western culture and way of life. These tools require translation and cultural adaptation in order to retain their validity and reliability in multi-cultural settings and internationally. Fox-Rushby and Parker (1995) expressed their concern about the relevance of translated versions of QOL instruments. In addition to the concerns about the quality of the translation process (Anderson, Aaronson, Bullinger, \& McBee, 1996), Hunt (1994) was concerned that cultural differences are not accounted for during the translation and the cultural adaptation process.

Price et al. (2009) argued that published guidelines for translations and cultural adaption include the methods to use to develop cross-culturally comparable versions of a QOL tool. While conventional translation strategies are simple and quick to carry out, they have limitations which can affect the applicability of the instrument to the new culture. It is important to recognise that close adherence to the original language tool during translation, does not automatically guarantee the validity of the new language version. There may be items that do not translate well or else do not have meanings for the groups targeted in the new language version; some items may be important for the culture for whom it was originally created but unimportant for the new one (Guyatt, 1993).

Health related QOL is basically subjective and shaped by persons' culturally determined views of health. Although, the biological structure of all human being is similar. However, their cultural contexts, values and belives about health and wellbeing are often differe. Therefore, whenever a comparison is drawn between the health related QOL of patients from different cultures or groups using self-administered tools culture can influence how QOL is viewed. (Maramaldi, Berkman, \& Barusch, 2005). Threats to questionnaire validity arise when they are used in cultures other than the ones they were developed in (Rajkumar \& Kumar, 1996). Quantitative methods require validity checks to translated tools to ensure they generate useful information regarding 
the practices and conditions of people. An ethnographic method such as the focus group is recommended as a way of having the new population look at the domains of a QOL tool to advise on how to make them culturally appropriate.

Health related QOL measures the impact of health or illness on one's ability to function, combined with their perception of physical, mental, and social well-being (Coons, Rao, Keiner, \& Hays, 2000). Furthermore, patient's perception of these areas of QOL is directly related to their cultural background, values, beliefs, and judgments. Campos and Johnson (1990) suggested that perceptions of QOL are shaped by values rooted in a person's culture. A person's culture influences their attitudes toward, and views about physical health and illness and mental health (Fayers et al., 1997; Gonzalez-Calvo, Gonzalez, \& Lorig, 1997; King et al., 1997). However, culture is dynamic and can change at both the individual and the group level because people exposed to different ideas (Vega, 1992).

\subsubsection{Methods of translations and cross-cultural adaptation of QOL tools}

Warnecke et al. (1996) in his study concluded that to use QOL as an outcome measure requires understanding how cultural, ethnic, religious and other values influence judgments about QOL. Comparison of research findings undertaken in different cultures could be misleading unless common methodologies are adopted. The danger of distortion could be further compounded by differences in languages and cultural background of the subjects (Sartorius \& Kuyken, 1994). In order to achieve accurate, cross-culturally comparable versions of QOL tools, formal methods of translation and adaptation need to be applied. The process ideally should involve a linguistic translation where the tool is translated into the new language and a cultural translation where the translated tool is examined and then adjusted appropriately for the cultural context of the new language. Having completed this evaluation process to ensure that the newly translated tool is comparable with the original tool is reviewed (Price et al., 2009). Price et al., developed a process to achieve accurate cross-cultural translations of a disease-specific QOL measure, using five steps: 1) forward translation, 2) backward translation, 3) review of source and final translated version, 4) pretesting for equivalence in source and final documents, and 5) an international consensus meeting. Other methods for reviewing the quality of translation and validating tool for a particular context are bilingual review, assessing readability, and pilot testing (Martinez, Ainsworth, \& Elder, 2008). 
Stewart and Napoles-Springer (2000) recommend that cultural adaptation of the tools should include three concepts; conceptual equivalence, cultural equivalence and linguistic equivalence. Conceptual equivalence means the terms and concepts for the measured items should have the same meaning in each culture. Stewart and NapolesSpringer recommend that terms be explained to ensure similarity between measures. Cultural equivalence is about ensuring measures capture the same for different populations. Achieving cultural equivalence involves back-translating the items, pilot testing with the target population, and comparing these results with the intent of the original tool. The meaning of cultural equivalence is debated (Herdman, Fox-Rushby, \& Badia, 1997). Van Widenfelt, Treffers, De Beurs, Siebelink and Koudijs (2005) recommended that for a measure to be culturally equivalent across different populations, the people should have the following: Firstly, shared norms such as socially expected behaviours, shared beliefs, ideas and assumptions about the world, and, shared values and expectations about what is right or wrong.

Linguistic equivalence is about the words and grammar having similar meanings across different cultures and languages. Translators and researchers should translate the meaning of a survey, rather than the exact words. It is important that the items of the original and the translated surveys have similar meanings (Geisinger, 1994). In general, QOL measures should be applied to multiple cultures, all items should be translated and culturally adapted so that the translated version has a natural flow, and captures the same meanong across cultures (Martinez et al., 2008).

As part of cultural adaptation of the QOL tools some researchers have altered or deleted items in QOL tools to make them culturally appropriate to the intended populations. Yildirim et al. (2007) in their translation and cross-cultural adaptation of the Diabetic Quality of life (DQOL) tool to use with 45 Turkish university students deleted the following item from the instrument "How often does your diabetes interfere with your sex life?" because sex was treated as prohibited in this group and respondents assumed would be offended and uncomfortable answering this question. Rajkumar and Kumar (1996) translated and culturally adapted the WHOQOL tool to the Indian culture; questions relating to sexual satisfaction and functioning always tended to draw answers which are socially desirable. However, responses from unmarried girls to items on heterosexual behaviour are considered offensive as they go against their value system. Therefore, these questions were deleted from the questionnaires given to the unmarried girls who participated in the study (Rajkumar \& Kumar). 
QOL tools need to have population normative values to provide a standard by which scores from local and international studies can be compared. The investigators need to know, what the average scores for the healthy population who live in the same area of those under study. Establishing normative data are important in interpreting scale scores of particular study population. The normative values should be derived from unbiased randomly selected stratified representative sample from all regions in the country (Bowling, Bond, Jenkinson, \& Lamping, 1999). The comparison should enable the investigators to decide whether the scores obtained from their study were above or below those of general population. Both national and regional norms are required. National norms are vital to make a comparison with different datasets of patients suffering from different health disorders and / or to make comparisons with other international studies. However, the regional norms are required also to give a true picture about the variations in health status of different geographical areas within the country (Bowling et al.,).

When examining the translation and the cultural adaptations of $\mathrm{QOL}$ tools careful attention should be paid to whether people's understanding and interpretation of the questions is likely to be influenced by their cultures (Angel \& Cronfein, 1988). The way how people from different cultures value health and perceive illness and death is different. For example, UAE and Jordanian Muslim people value health and perceive death as part of life and look at illness as a test from God. In contrast, people from other cultures may not link health and illness to any religious or spiritual practices. Response editing is commonly encountered phenomenon that we need to pay attention to. In some cultures people might chose more socially desirable responses when answering questions related to healthy life styles (Bachman, O'Malley, \& Johnston, 1984). In Arabic culture, asking people superficially about their health may grant a "good" response, but when asked in more details about their health you may discover the opposite. Mapping judgments on to a common metric scale is also influenced by culture. For example, African-American and Hispanic survey respondents have been found to be less likely than Anglo-American to qualify their answers on rating scales, whereas Asians are less likely to prefer extreme responses (Bachman et al.). 


\subsection{Quality of life tools}

The literature is very rich in a range of QOL tools that have been used in different research areas. Examples of tools used to research dialysis patients are summarised in Table 3.1.

This research used the SF-36 for both samples and used the QOL Index dialysis version for dialysis patients and the QOL Index generic version for participants from the community. The SF-36 is a general tool. In contrast, the QOL Index is a diseasespecific tool. It measures satisfaction and importance of determinants of QOL. The dialysis version of the QOL Index was developed to be used for people on dialysis as it has specific questions related to kidney failure. While the QOL Index generic version was developed for people from the general population. Both tools have well established reliability and validity studies. The Arabic version of the QOL Index was translated by Halabi (2006).

The rationale for choosing a particular tool for research is shaped by the research questions, the population and the research consideration. The question in this research concerned the QOL of people on dialysis in UAE. The tools used therefore had to be applicable to people with chronic illness, reliable, and applicable to people from general population. As most people in UAE speak either English or Arabic, the tools had to be available in both languages. 
Table 3.1 Examples of general tools used to measure quality of life

\begin{tabular}{|c|c|c|c|c|c|}
\hline Name (source) & Dimensions explored & Description & $\begin{array}{l}\text { How } \\
\text { administered }\end{array}$ & Population & $\begin{array}{l}\text { Other } \\
\text { comments }\end{array}$ \\
\hline $\begin{array}{l}\text { WHO QOL-BREF } \\
(2006)\end{array}$ & $\begin{array}{l}\text { Consists of } 4 \text { dimensions: } \\
\text { physical health, psychological } \\
\text { health, social relationships } \\
\text { and environment. }\end{array}$ & $\begin{array}{l}\text { Consists of } 26 \text { items that ask individuals to } \\
\text { rate their subjective perception of life in the } \\
\text { context of the culture and value system. } \\
\text { There are five Likert-type response options, } \\
\text { ranging from "very dissatisfied" (score of } 1 \text { ) } \\
\text { to very satisfied" (score of } 5 \text { ), with higher } \\
\text { scores denoting higher QOL }\end{array}$ & $\begin{array}{l}\text { Administered by } \\
\text { clinician }\end{array}$ & $\begin{array}{l}\text { General } \\
\text { population }\end{array}$ & $\begin{array}{l}\text { Not self } \\
\text { administered } \\
\text { affects its } \\
\text { reliability }\end{array}$ \\
\hline $\begin{array}{l}\text { SF-36 (Ware et al., } \\
\text { 1993) }\end{array}$ & $\begin{array}{l}\text { Consists of } 8 \text { dimensions: } \\
\text { physical functioning, physical } \\
\text { role limitation, bodily pain, } \\
\text { mental health, mental role } \\
\text { limitations, social functioning, } \\
\text { vitality and general health } \\
\text { perceptions }\end{array}$ & $\begin{array}{l}\text { Consists of } 36 \text { questions that assess } 8 \\
\text { objective dimensions of QOL, which } \\
\text { converge to } 2 \text { summary measures: physical } \\
\text { health and mental health }\end{array}$ & $\begin{array}{l}\text { Interviewer, } \\
\text { computer, or self } \\
\text { administered }\end{array}$ & $\begin{array}{l}\text { General } \\
\text { population } \\
\text { surveys, clinical } \\
\text { trials and clinical } \\
\text { practice }\end{array}$ & $\begin{array}{l}\text { Used in many } \\
\text { studies }\end{array}$ \\
\hline $\begin{array}{l}\text { Nottingham Health } \\
\text { Profile (Jensen, et } \\
\text { al. 1997) }\end{array}$ & $\begin{array}{l}\text { Consists of } 6 \text { dimensions: } \\
\text { Emotional reactions, social } \\
\text { isolation, physical mobility, } \\
\text { pain, energy and sleep }\end{array}$ & $\begin{array}{l}\text { Consists of } 38 \text { statements that subjects } \\
\text { answer with yes or no. Responses are given } \\
\text { a score. }\end{array}$ & $\begin{array}{l}\text { Self } \\
\text { administered }\end{array}$ & $\begin{array}{l}\text { Intended for } \\
\text { primary } \\
\text { healthcare to } \\
\text { evaluate } \\
\text { perceived } \\
\text { distress across } \\
\text { various } \\
\text { populations. } \\
\text { Proposed users: } \\
\text { surveys and } \\
\text { intervention } \\
\text { studies in } \\
\text { combination with } \\
\text { clinical interview. }\end{array}$ & $\begin{array}{l}\text { Statements are } \\
\text { unequally } \\
\text { distributed } \\
\text { among the six } \\
\text { categories. Not } \\
\text { found to be } \\
\text { effective in } \\
\text { picking up } \\
\text { changes in status }\end{array}$ \\
\hline $\begin{array}{l}\text { EQ-5D-QOL } \\
\text { (Essink-Bot et al., } \\
\text { 1993) }\end{array}$ & $\begin{array}{l}\text { Consists of } 6 \text { dimensions: } \\
\text { mobility, self-care, usual } \\
\text { activities, anxiety/ depression, } \\
\text { pain / discomfort, and self } \\
\text { evaluation of overall health }\end{array}$ & $\begin{array}{l}\text { Consists of } 5 \text { items (3 response options per } \\
\text { dimension), plus a visual analogue scale. } \\
\text { Standardised generic measures of } \\
\text { describing health statistics }\end{array}$ & $\begin{array}{l}\text { Self } \\
\text { administered } \\
\text { diary }\end{array}$ & $\begin{array}{l}\text { Patient health } \\
\text { status }\end{array}$ & $\begin{array}{l}\text { Derived from } \\
\text { tools, including } \\
\text { the sickness } \\
\text { Impact profile } \\
\text { and Nottingham } \\
\text { Health Profile } \\
\text { tool }\end{array}$ \\
\hline
\end{tabular}




\begin{tabular}{|c|c|c|c|c|c|}
\hline Name (source) & Dimensions explored & Description & $\begin{array}{l}\text { How } \\
\text { administered }\end{array}$ & Population & $\begin{array}{l}\text { Other } \\
\text { comments }\end{array}$ \\
\hline $\begin{array}{l}\text { QOL Index (Ferrans } \\
\text { \& Powers, 1984) }\end{array}$ & $\begin{array}{l}\text { Consists of } 4 \text { dimensions: } \\
\text { health and functioning, } \\
\text { psychological/spiritual, social } \\
\text { and economic, and family } \\
\text { dimensions }\end{array}$ & $\begin{array}{l}\text { Consists of } 34 \text { questions that measure } \\
\text { subjectively the satisfaction of certain } \\
\text { aspects of life and another } 34 \text { questions that } \\
\text { measure importance of those aspects to the } \\
\text { person answering the tool. A number of } \\
\text { versions of the QOL Index have been } \\
\text { developed for use with various disorders and } \\
\text { the general population }\end{array}$ & $\begin{array}{l}\text { Self } \\
\text { administered }\end{array}$ & $\begin{array}{l}\text { Various disorders } \\
\text { and the general } \\
\text { population }\end{array}$ & $\begin{array}{l}\text { Easy to use, } \\
\text { practical }\end{array}$ \\
\hline
\end{tabular}


Given no research had previously been located on QOL in UAE; consideration was needed as to the most applicable tool. As there was no easy answer to this question, two tools were used in this study. Each tool captures different dimensions of QOL. The researcher adopted Mangione's (2002) six criteria for selecting the QOL tools.

1. The tool should be reported in the literature

2. The tool should measure aspects of life that are of interest

3. The tool should have been tested for psychometric properties (validity and reliability)

4. The tool should not be cost prohibitive

5. The tool should be easy to administer (doable)

6. The tool should be easy to score and interpret (scoring and interpretation guidelines).

Each of these points is discussed separately in relation to the selection criteria for the SF36 and QOL Index tools. "Validity is the extent to which a test such as QOL tool measures what it claims to measure. It is vital for a test to be valid for the results to be accurately applied and interpreted" (Cherry, 2010). "Reliability means dependability or consistency of it suggest that the same thing is repeated or recurs under the identical or very similar conditions" (Neuman, 2006, p. 188). The numerical results produced by an indicator should not vary because of the characteristics of the measurement process or measurement instrument itself.

\subsubsection{SF-36}

The SF-36 is a general tool, developed to be used on all populations irrespective of health or illness. It is one of the most commonly used measures to study the QOL in dialysis patients (Liem, Bosch, Arends, Heijenbrok-Kal, \& Hunink, 2007; Unruh \& Hess, 2007). In addition to the English version developed for use in the United States, it is approved and available through the Boston-based International Quality of Life Assessment project (IQOLA) for other settings. The SF-36 version 2 is currently available in more than 120 translations (Quality Metric tools, 2011). This study has used the SF-36 tool version 1 because at the time of data collection in June 2007 the SF-36 tool version 1 was available 
in Arabic language. Version 1 had been used by several researchers in the Middle East (Khoudri, Ali Zeggwagh, Abidi, Madani, \& Abouqal, 2006: Sabbah, Drouby, Sabbah, RetelRude, \& Mercier. 2003). There were no normative values of the SF-36 for the UAE at the time of this research. The SF-36 tool consists of 36 items that assess eight dimensions (subscales). A summary of the SF-36 dimensions is outlined in Table 3.2.

Table 3.2 Description of the dimensions of SF-36

\begin{tabular}{|l|l|}
\hline Dimensions & Summary of contents \\
\hline Physical Function (PF) & $\begin{array}{l}\text { Extent to which health limits physical activities such as walking, self- } \\
\text { care, climbing stairs, lifting and exercises }\end{array}$ \\
\hline Role Physical (RF) & $\begin{array}{l}\text { Extent to which physical health interferes with work including } \\
\text { accomplishing less and difficulties in performing activities }\end{array}$ \\
\hline Body Pain (BP) & Intensity of pain and its effect on work \\
\hline General Health (GH) & Personal evaluation of health, including current health and health outlook \\
\hline Vitality (VT) & Feeling energetic versus feeling tired and worn out \\
\hline Social Functioning (SF) & $\begin{array}{l}\text { Extent to which physical health or emotional problems interfere with } \\
\text { social activities }\end{array}$ \\
\hline Role Emotional (RE) & $\begin{array}{l}\text { Extent to which emotional problems interfere with work including } \\
\text { decreased time spent on activities, accomplishing less }\end{array}$ \\
\hline Mental Health (MH) & $\begin{array}{l}\text { General mental health, including depression, anxiety, behavioural- } \\
\text { emotional control }\end{array}$ \\
\hline
\end{tabular}

The number of questions directed to each health concept range from two (for social functioning and bodily pain) to 10 (for physical functioning). The number of response options per question range from two (no, yes) to six (none, very mild, mild, moderate, severe, and very severe). Normalised scores representing overall physical functioning and mental functioning are calculated from the individual scales and are presented as the Physical Component Scale (PCS) and Mental Component Scale (MCS). The PCS includes the dimensions of Physical Function (PF), Role Physical (RF), Body Pain (BP), and General Health (GH). The MCS is composed of the Vitality (VT), Social Functioning (SF), Role Emotional (RE), and Mental Health (MH) subscales. Scores are assembled using the Likert method for summated ratings. All raw scale scores are linearly converted to a 0 (worst possible health status or QOL) to 100 (best possible health status or QOL). The score of the subgroup as well as the final global score of the SF-36 ranges between 0 and 100, respectively (Ware \& Sherbourne, 1992). 


\subsubsection{Critique of the SF-36}

While the SF-36 may be used to compare the health status of those on dialysis with those with other chronic illnesses or healthy population, the SF-36 has substantial floor and ceiling effects, and limited longitudinal measurement precision (Unruh, Weisbord, \& Kimmel, 2005). Moreover, the instrument does not measure other dimensions such as income, sleep disturbances, pain, body image, patient satisfaction with care, and sexual function that may be important to an individual's sense of well-being and QOL. Andresen and Meyers (2000) argued that the SF-36 was primarily developed to measure health, and is therefore erroneously used by researchers it to measure QOL or health-related QOL. Moons (2004) questioned why some researchers refer to this instrument as a QOL instrument when it really measures perceived health. The usage of the SF-36 to measure QOL may therefore not reflect the actual perceived QOL of the respondents', but this tool is useful for studying the physical and the mental components of QOL. Ware and Gandek (1998) highlighted that an extensive effort was made to develop culturally appropriate versions of the SF-36. For example there are four different versions in the English language. This indicates that straight language translation can be in adequate. A summary of the selection criteria for the SF-36 are summarised in Table 3.3.

\subsubsection{Quality of Life Index tool}

The QOL Index was developed by Ferrans and Powers in 1984 to measure QOL in terms of satisfaction with life. The dialysis version of the QOL Index has 34 items and the generic version QOL Index is 33 items. Both tools are in two parts, the first measure satisfaction with various dimensions of life and the second measures the importance of the same dimensions. The parts composed of five subscales: health and functioning, social and economic, psychological and spiritual, and family. Subjects respond to each item on a 6point scale, ranging from "very satisfied" to "very dissatisfied" and "very important" to "very unimportant". The score range is $0-30$, with the higher score representing better QOL. The evidence for psychometric properties of the QOL Index has been documented (Ferrans \& Powers, 1992). 
Table 3.3 Assessment of the SF-36 against the selection criteria

\begin{tabular}{|l|l|}
\hline Criteria & Description \\
\hline $\begin{array}{l}\text { The tool should } \\
\text { be reported in the } \\
\text { literature }\end{array}$ & $\begin{array}{l}\text { Has been used in over 2,000 published research studies (iii). It has been } \\
\text { judged to be the most widely evaluated generic patient assessed health } \\
\text { outcome measure (i). The SF-36 is sensitive to changes in health in general } \\
\text { populations (ii). }\end{array}$ \\
\hline $\begin{array}{l}\text { The tool should } \\
\text { measure aspects } \\
\text { of life that are of } \\
\text { interest (validity) }\end{array}$ & $\begin{array}{l}\text { Has been used in various countries in clinical practice with hemodialysis } \\
\text { patients and found to be a comprehensive, valid, reliable, and potentially useful } \\
\text { scale for evaluating patients health-related QOL (iii). Addresses eight major } \\
\text { dimensions: physical functioning, role-physical, bodily pain, general health } \\
\text { perceptions, vitality, social functioning, role-emotional, and mental health. }\end{array}$ \\
\hline $\begin{array}{l}\text { The tool should } \\
\text { have been tested } \\
\text { for psychometric } \\
\text { properties }\end{array}$ & $\begin{array}{l}\text { Reported internal consistency, test re-test reliability, alternate form reliability, } \\
\text { content, construct and discriminate validity (iv). Power analysis has been } \\
\text { constructed with the eight scales. Appropriate sample sizes could be selected } \\
\text { for a variety of research designs with a variety of effect sizes. The scales have } \\
68 \%, 90 \% \text { and 95\% confident intervals determined for the norms of the US } \\
\text { population (iv) the Arabic translation of the tool has been validated (vi) }\end{array}$ \\
\hline $\begin{array}{l}\text { The tool should } \\
\text { not be cost } \\
\text { prohibitive }\end{array}$ & $\begin{array}{l}\text { Permission to use SF-36 was granted by Quality Metric Incorporated for } \\
\text { minimal cost. }\end{array}$ \\
\hline $\begin{array}{l}\text { The tool should } \\
\text { be easy to } \\
\text { administer }\end{array}$ & $\begin{array}{l}\text { Is a self-report scale, designed to measure the QOL for different patient } \\
\text { populations (v). It can be self-administered by persons 14 years of age and } \\
\text { older. Can be administered by paper and pen or interview. Time to complete } \\
10-20 \text { minutes. }\end{array}$ \\
\hline $\begin{array}{l}\text { The tool should } \\
\text { be easy to score } \\
\text { and interpret }\end{array}$ & $\begin{array}{l}\text { Scoring can be done using SPSS programme. Scores range from 0 - 100, with } \\
\text { higher scores indicating higher QOL, it is possible to compare results across } \\
\text { different versions in different languages. Sub-scale analysis can be done. }\end{array}$ \\
\hline
\end{tabular}

Sources:

i. Garratt, A., Schmidt, L., Mackintosh, A., \& Fitzpatrick, R. (2002). Quality of life measurement: Bibliographic study of patient assessed health outcome measures. British Medical Journal, 15324(7351), 1417-1422.

ii. Hemingway, H., Stafford, M., Stansfield, S., Shipley, M., \& Marmot, M. (1997). Is the SF-36 a valid measure of change in population health? Results from the Whitehall II study. British Medical Journal, 315(7118), 1273-1279.

iii. Mingardi, G. et al. (1999). Health-related quality of life in dialysis patients. A report from an Italian study using the SF-36 health survey. Nephrology Dialysis Transplantation Journal, 14(6), 1503-1510.

iv. Ware, J. (1993). SF-36 health survey manual \& interpretation guide. Boston, Mass: The Medical Outcome Trust.

v. Sayin, A., Mutluay, R., \& Sindel, S. (2007). Quality of life in hemodialysis, peritoneal dialysis, and transplantation patients. Transplant Proceedings Journal, 39(10), 3047-3053.

vi. Khoudri, I., Ali Zeggwagh, A., Abidi, K., Madani, N., \& Abouqal, R. (2006). Measurement properties of the Short Form 36 and health-related quality of life after intensive care in Morocco. Acta Anaesthesiologica Scandinavica Journal, 51(2), 189-197.

This study used the QOL Index dialysis version III for dialysis patients and the generic version III for the participants from the community. The dialysis version is 68 questions in total and the generic version is 66 questions. Most questions in both versions are the same; however, there are four different questions in the dialysis version and two in the generic version. These questions belong to the health and functioning subscale. The 
dialysis version relate to kidney failure. The first question asks about the satisfaction with the likelihood of getting a kidney transplant. The second question asks about the importance of getting a kidney transplant. The third question asks about the satisfaction with the changes they have had to make in their life because of kidney failure (such as diet and need for dialysis). The fourth question asks about the importance of the changes they have has to make in their life because of kidney failure. In the generic version the first different question asks about the satisfaction with the amount of pain had and the second question asks about the importance of having no pain. Table 3.4 summarises the criteria for selecting the QOL Index.

\subsubsection{Critiquing the QOL Index}

The QOL was defined by Ferrans (1996, p.15) as "a person's sense of well-being that stems from satisfaction or dissatisfaction with the areas of life that are important to him/her". This definition highlights the way Ferrans and Powers (1992) conceptualised the term QOL. The QOL concept in their view is subjective in nature. Consequently, people's perceptions of their own QOL are a true reflection of the quality of their life.

The QOL Index has many positive aspects. It measures both a persons' satisfaction with and its importance several aspects of life at the time of administration. The scores are intended to reflect satisfaction with the aspects of life that is valued by the individual, so patients will decide how satisfied with certain aspects of life they are and rate the importance of these aspects accordingly. However, the patient population used to validate the QOL Index was primarily well-educated middle and upper middle class individuals (Warnecke et al., 1996). These populations did not include African American, Hispanic or Arabic patients; those patients often tend to be less educated and have different cultural backgrounds. The misrepresentations due to differences in cultural understanding of the questions and the internalised interpretations have been described as "category fallacy" (Warnecke et al.). It is a common problem with most QOL tools. 
Table 3.4 Assessment of the QOL Index against the selection criteria

\begin{tabular}{|c|c|}
\hline Criteria & Description \\
\hline $\begin{array}{l}\text { The tool should } \\
\text { be reported in the } \\
\text { literature }\end{array}$ & $\begin{array}{l}\text { Reported in more than } 100 \text { published studies. A number of versions of the } \\
\text { QOL Index have been developed for use with various disorders and the } \\
\text { general population. It can reflect the positive or negative influence of QOL (i, } \\
\text { ii, iii, iv, vi). }\end{array}$ \\
\hline $\begin{array}{l}\text { The tool should } \\
\text { measure aspects } \\
\text { of life that are of } \\
\text { interest (validity) }\end{array}$ & $\begin{array}{l}\text { QOL Index differentiates clearly between QOL and health, unlike health- } \\
\text { related QOL tools which measure the health status and refer to it as QOL. It } \\
\text { measures QOL in terms of satisfaction with life. Used to study the QOL of } \\
\text { healthy and unhealthy populations. Addresses } 5 \text { major dimensions: health } \\
\text { and functioning, social and economic, psychological and spiritual, and family. } \\
\text { Consists of two parts: the first measures satisfaction with various aspects of } \\
\text { life and the second measures importance of the same aspects. }\end{array}$ \\
\hline $\begin{array}{l}\text { The tool should } \\
\text { have been tested } \\
\text { for psychometric } \\
\text { properties }\end{array}$ & $\begin{array}{l}\text { Psychometric properties of the Arabic versions was established (v). The } \\
\text { translated version of the Arabic QOL Index demonstrated a high degree of } \\
\text { accuracy of translation and estimates of content validity. The results revealed } \\
\text { high estimates of reliability for the generic version of } 0.94-0.97 \text {, and the } \\
\text { dialysis version } 0.93(\mathrm{v}) \text {. }\end{array}$ \\
\hline $\begin{array}{l}\text { The tool should } \\
\text { not be cost } \\
\text { prohibitive }\end{array}$ & $\begin{array}{l}\text { Permission to use QOL Index was granted for free from the author, Providing } \\
\text { acknowledgment the authors in all future publications. }\end{array}$ \\
\hline $\begin{array}{l}\text { The tool should } \\
\text { be easy to } \\
\text { administer }\end{array}$ & $\begin{array}{l}\text { Can be self-administered by people with reading level of fourth grade. Can be } \\
\text { administered by paper and pen or interview. Time to complete } 10-20 \text { minutes. }\end{array}$ \\
\hline $\begin{array}{l}\text { The tool should } \\
\text { be easy to score } \\
\text { and interpret }\end{array}$ & $\begin{array}{l}\text { Participants were asked to rate their satisfaction with some elements of their } \\
\text { life on a scale from one to six. One means very dissatisfied and six means } \\
\text { very satisfied. Participants were also asked to rate the importance of the } \\
\text { same elements according to how important those elements were to them in a } \\
\text { scale from one to six. One means very unimportant and six means very } \\
\text { important. The total scores for all versions range from 0-30. It has step-by- } \\
\text { step scoring instructions available on the author's website. }\end{array}$ \\
\hline \multicolumn{2}{|r|}{ ( } \\
\hline \multicolumn{2}{|c|}{$\begin{array}{l}\text { i. Ferrans, C. (1996). Development of a conceptual model of quality of life. Scholarly Inquiry for Nursing } \\
\text { Practice Journal, 10(3), 293-304. } \\
\text { ii. Ferrans, C.E. (1990a). Quality of life: Conceptual issues. Seminars in Oncology Nursing, 6(4), 248- } \\
\text { 254. } \\
\text { iii. Ferrans, C., \& Powers, M. (1992). Psychometric assessment of the Quality of Life Index. Research in } \\
\text { Nursing and Health, 15(1), 29-38. } \\
\text { iv. Ferrans, C., \& Powers, M. (1985). Quality of life index: Development and psychometric properties. } \\
\text { Advances in Nursing Science, 8(1), 15-24. } \\
\text { v. Halabi, J. (2006). Psychometric properties of the Arabic version of Quality of Life Index. Journal of } \\
\text { Advanced Nursing, 55(5), 604-610. } \\
\text { vi. Warnecke, RB., Ferrans, CE., Johnson, TP., Chapa-Resendez, G., O'Rourke, DP., Chávez, N., et al. } \\
\text { (1996). Measuring quality of life in culturally diverse populations. Journal of the National Cancer } \\
\text { Institute Monographs, (20), 29-38. }\end{array}$} \\
\hline
\end{tabular}




\subsubsection{Points related to critiquing QOL tools}

When analysing the results of any QOL tool, researchers need to think about category fallacy. Category fallacy results from the failure to distinguish between the concepts that are truly universal and accepted across multiple culture groups and the concepts that have meaning only within a specific cultural group or socioeconomic context (Warnecke et al., 1996). Furthermore, the culture and the interpretation differences of the questions are likely to influence how respondents understand questions dealing with the QOL (Angel \& Cronfein, 1988). Sometimes the language into which a question is translated does not contain the right concept (Angel \& Thoits, 1987).

Information retrieved from memory and judgment formation is an important aspect of attitude formation toward judging QOL. The importance of events and satisfaction with one's current life status are translated into an assessment of QOL (Ferrans, 1990). Most often when a respondent is asked about the value attributed to a life aspect such as an event, experience or action, the response is a synthesis of information retrieved from memory about relevant experience. The more frequently such information is used, the more accessible it is in memory; hence, the more readily it is used for developing judgment (Warnecke et. al., 1996). Questions about satisfaction and importance assumes that the individual has stored in memory relevant experiences that will be available for forming judgments about the importance of and current satisfaction with the life aspect related to each question. If there are no memories of specific and relevant events to cue the person's responses, the actual responses to questions about how much the person is satisfied with a particular life aspect or how important it is may be based on the motivation to be a "good respondent". Hence the responses may be subject to editing rather than reflecting the respondents true assessment.

Response editing is a commonly encountered phenomenon when survey respondents feel that certain answers are more socially desirable than others. For example, socially desirable such as exercising and nutrition are frequently over-reported, whereas undesirable behaviours as drinking alcohol or smoking are frequently under-reported (Bachman, O'Malley, \& Johnston, 1984). The validity of scales requires a common frame of reference for mapping judgments on to a common metric. For example, African- 
American and Hispanic survey respondents have been found to be less likely than AngloAmerican to qualify their answers on rating scales, whereas Asians are less likely to prefer extreme responses (Bachman et al.). Preference for extreme versus cautious response styles has been interpreted as being a consequence of cultural variation in emphasis on sincerity versus modesty and social interaction (Bachman et al.).

\subsection{Conceptual framework for this study}

\subsubsection{Background}

Although a considerable number of studies have been conducted examining QOL among dialysis patients, the literature review revealed minimum use of guiding conceptual frameworks that could be used to examine the subjective and objective dimensions of QOL systematically. Researchers need to explore different conceptual frameworks before using QOL tools to inform data collection and analysis (Laliberte-Rudman, Hoffman, Scott, \& Renwick, 2004; Perez et al., 2007). Some studies have focused on measurement issues and psychometric properties (Lowenstein, 2007; Perez et al., 2007) leaving conceptual issues unaddressed. Using a conceptual framework is important in providing a structure to investigate the contributing factors to QOL and how these factors influence the outcomes of dialysis patients. One needs to look at the wider aspects of the QOL that may have an influence in determining the factors that contribute to QOL. Satisfaction with life is influenced by different factors such as spirituality, availability of family and social support (Patel et al., 2002; Thong, Kaptein, Krediet, Boeschoten, \& Dekker, 2007). In the UAE, the majority of the population including those on dialysis are Muslims. The influence of religion, spirituality, and family support may have an impact on the QOL. Therefore, the conceptual framework in this study is looking at health and illness from an Islamic prospective.

Muslims, the followers of Islam live almost everywhere in the world. Not all Muslims live in the Middle East. Moreover, the Middle East is not only inhabited by Arabs, there are other ethnic groups who live there such as Turks and Iranians. So, there is more than one culture in the Middle East and the Arab culture is one of them. It is important to highlight that not all Arabs are Muslims. Large numbers of Arab Christians are found in the Southwest Asia, particularly in Egypt, Syria, Lebanon, Jordan, Iraq, West Bank and Gaza 
Strip. Despite of the presence of Arab Christians and other religious groups in UAE, the overriding cultural norm and values of the country are influenced by Islamic principles.

The framework was developed because the the available conceptual frameworks in the literature were Western based and were not applicable for UAE where religion, spirituality and family are particularly important. Therefore, interpreting findings based on the available frameworks may lead to confusion and misleading results. This conceptual framework was developed to guide the research data collection and interpretation. The framework considered the likely subjective and objective dimensions of QOL in UAE settings, and examined the literature concerning the physiological, psychosocial, religious (beliefs), cultural, physical and mental health aspects of QOL. The conceptual framework is illustrated in Figure 2.1.

\subsubsection{Content of the conceptual framework}

The conceptual framework consists of two major levels. The first level is related to the influence of Islamic culture and society on QOL. The second level is related to the individual's characteristics that contribute to their overall QOL such as religion, family, physical health and life events. The culture and society influence people's life in different ways. Each society has its own culture. Within the society people belong to sub-groups and these sub-groups interact differently compared with others. Islam as a religion sets some rules and regulations for how should people interact and live in the community. These rules and regulations are considered as general guidelines that govern people's behaviour and relationships. The level of adherence to Islamic teaching can vary depending on the degree of religiosity among individuals (Barhem, Younies, \& Muhamad, 2009). Religious people follow the guidelines strictly, so they build strong and friendly relationships with others based on respect and caring. Those individuals are expected to have better QOL because they accept what they have and at the same time care for others. 
Figure 3.1 Diagram of the conceptual framework

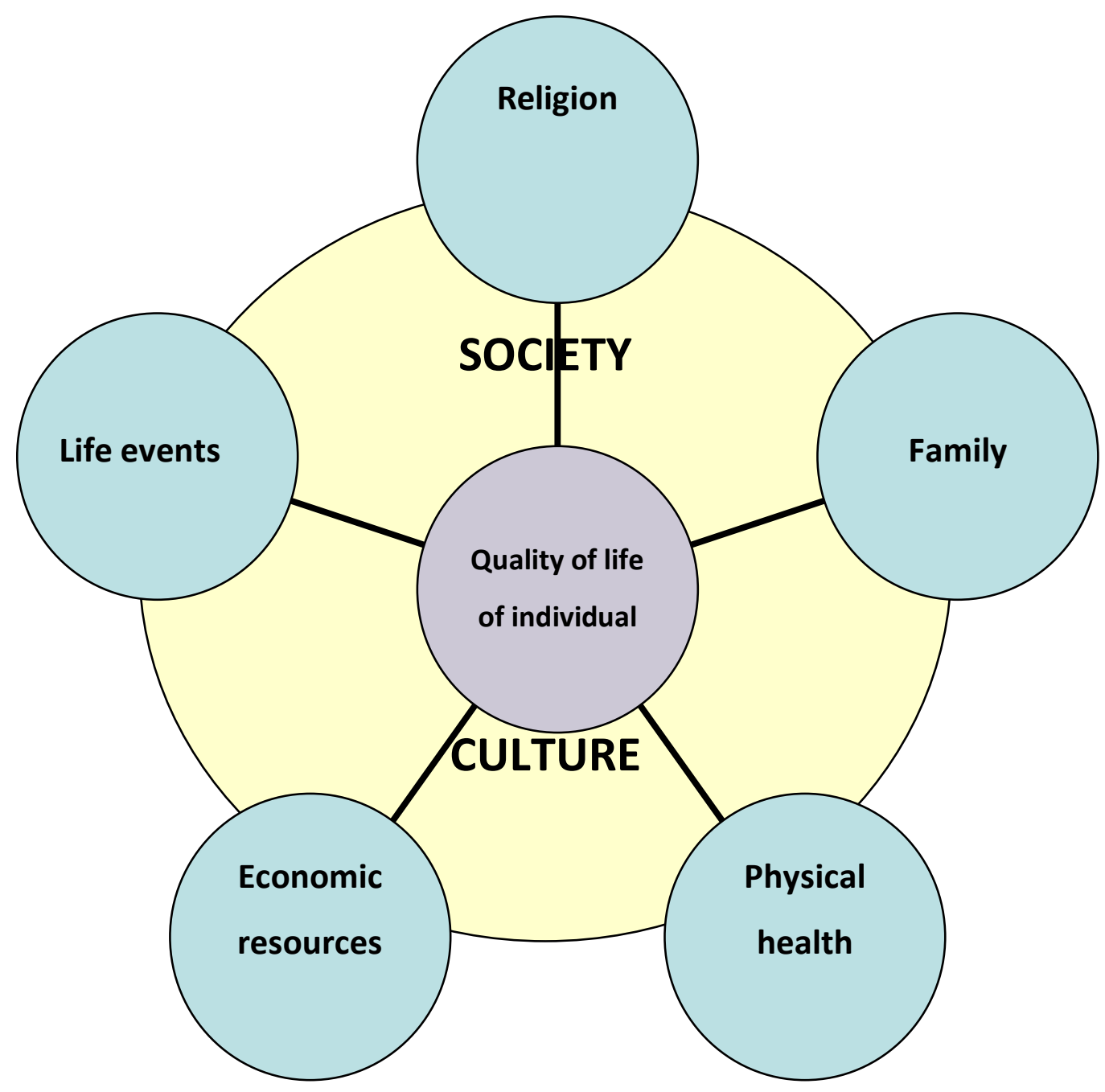

\subsubsection{Culture and society}

There are different views on QOL and these views may be partly culturally driven (Scott et al., 2008). QOL reported by the people is based on personal perceptions, impressions, feelings and reactions. The QOL scores are therefore embedded in people's cultural reality. Subcultures differ from the main culture in that they may not hold all the values of their main culture; they share beliefs according to primary and secondary cultural characteristics. Primary characteristics include nationality, race and religious affiliation, 
while secondary characteristics include educational status, socioeconomic status and gender issues (Purnell \& Paulanka, 2005). The influence of cultural differences in Islamic communities and the subculture phenomenon is very obvious. Not all Muslims have the same culture but they share general rules based on a common religion (Shuraida, 2001). Muslim population around the globe is not a homogeneous group. Rather, there is diversity of cultural and local customs. For many Muslims, racism is incomprehensible in Islamic thought and practices. The Holy Qur'an speaks of human equality in the following terms: "O mankind we have created you male and female, and have made you into nations and tribes so that you may know each other. Lo! The noblest of you in the sight of Allah is the one with piety or the one who is pious" (Al-Hujurat 49:13).

People living in the UAE have different cultures. The majority $(57 \%)$ of the population is from South East Asian countries. Arabs constitute up to 21\%, and UAE Nationals make up $11 \%$ of the population (Pejman, 2007). Each of these groups has their own subcultures but all of them live under a major culture that governs all the population. This culture is shaped by religion, tradition, and the political system. Culture and social norms shape the interaction between people in the community. The way people interact in the community is governed by the culture of the UAE. However, within the country itself there are subcultures. Arabs have a common language (Arabic) but there are dialectic differences that vary from country to country. Also, there is a formal language, which is mainly used in formal situations such as radio broadcasts and official correspondence. Islamic values and behavioural guidelines support and promote the health of the individuals, family and community; healthcare intervention should promote and maintain the cultural practices (Leininger, 1996). It was expected that the culture of the UAE would impact positively on the QOL of dialysis patients but there would be a stronger influence of the main cultures on how respondents perceived the quality of their own life.

In the UAE gender is likely to have an impact on the QOL as men and women have different roles to play in the UAE society and Middle Eastern societies. There are cultural factors that restrict women in the UAE such as not living alone, and not being fully independent life. Involvement of women in social activities may impact positively on the QOL as this may make them feel valued and foster their sense of belonging to the society. It was expected that male gender would have a positive impact on the total scores for the 
general population group due to the fact that there are fewer restrictions on men in the community. However, men who are dialysis patients, in Middle Eastern communities are also probably the bread-winners, therefore they are more likely to suffer because of poor financial status due to unemployment and this may be reflected in their QOL. Furthermore, it is likely that a change in role between dialysis patients and their partners would have occurred in terms of employment. The wife would have taken the role of being the breadwinner and the husbands' role in the family would change to be more dependent on the wife for most things. This would have a major impact on both men's and women's psychological status and could lead to intense feelings of powerlessness.

\subsubsection{Religion}

The concept of caring from Islamic perspectives is rarely discussed in Eurocentric nursing literature (Laird, de Marrais, \& Barnes, 2007). Caring from an Islamic perspective needs more clarification (Halligan, 2006). Salleh (1994) highlighted that caring in Islam through the practice of nursing means that consideration should be given to elements of gender, dress code, personal values, code of conduct and ethics, dietary requirements, family planning, health and safety and spiritual development.

The worldview of Muslim patients towards health, illness and death incorporates the vision of receiving illness and death with patience, meditation and prayers. Muslim patients generally understand that illness, suffering and dying are part of life and a test from Allah and nobody has control of how long they are going to live. The Holy Qur'an states "Be sure we shall test you with something of fear, hunger, some loss in wealth, lives or the produce (of your toil), but give glad tidings to those who patiently persevere" (Al-Baqarah 2:15). Islamic religion encourages people to live a healthy life style, seek treatment and visit and support those who are sick (financially and psychologically). When asked what actions are most excellent, Prophet Muhammad (PBUH) replied, "To gladden the hearts of human beings, to feed the hungry, to help the afflicted, to lighten the sorrow of the sorrowful, and to remove the sufferings of the injured (Bukhari)".

The literature indicates that the experience of different ethnicities and religious beliefs of people living on dialysis might be significantly different (Hicks et al., 2004; Kimmel et al., 2003; Patel et al., 2002; Unruh et al., 2004). In healthcare, there is some debate as to 
whether the predominant western paradigm of nursing care and management is applicable to Muslims (Halligan, 2006; Laird et al., 2007). Islamic teachings and practice use a holistic approach to meet the physical, spiritual, psycho-social and environmental needs of people and communities. Adib (2004) argued that Islamic medicine pays attention to holistic health (body and spirit), the individual and society. While, Ahmed (1999) emphasizes that Islam encourage the utilisation of all useful resources to help and treat all mankind.

There are different views of how the spiritual aspect is critically analysed in the nursing literature. From the western prospective, there are not always differences between religion and spirituality and often used interchangeably (Dyson, Cobb, \& Forman, 1997; Harrison, 1993). However, spirituality has a broader meaning than religion and includes more philosophical ideas about the meaning and the purpose of life, not every person who seeks self-actualisation and self-empowerment follow a particular religion or faith. Wright (1999) considered that spirituality is a combination of our values and belief systems that determine how we interact with the people around us, whereas religion is a pathway to follow when practising a particular faith. Rasool (2000) argued that some of the conceptual frameworks and care models lack a spiritual dimension. In Islam there is no difference between religion and spirituality. There is no spirituality without religious thoughts and practices, and the religion provides the spiritual path and a way of life (Rasool).

From a Muslim perspective, religion is the basic and fundamental reference in dealing with everyday life activities and a reference for solving conflicts between individuals. There is a variation amongst Muslims as to the degree of religiosity and the adapting of Islamic teachings and instructions in everyday activities. Religion shapes the way Muslims think and view different aspects of life including health and illness as well as their perception of their own QOL. Dialysis patients are expected to have different degrees of religiosity. Those who were highly committed to and followed religious instructions are expected to have a better overall satisfaction with life. However, this study did not look at the degree of religiosity among the selected sample. Given that people have different religious and cultural backgrounds people may have belonged to different religions which could influence their QOL. 


\subsubsection{Physical health}

Islamic religion promotes a healthy life style by directing people to maintain a balance between diet and physical activity. QOL research comparing healthy population with those with chronic illnesses have found that physical health and the degree of disability have a remarkable effect on people's perceptions of the quality of their life. Confounding factors such as the patient's overall physical condition, co-morbidity, time of assessment, psychosocial conditions, life events, financial resources, society, belief and demographics variables such as gender have to be considered when assessing or interpreting QOL. Body pain and physical limitation have a high impact on people's perception of their QOL as well as vitality. Having mental peace and a stable mental health will have a major impact on people's perception of their QOL. Many Muslim patients look at illnesses as compensation for their wrong doing, and death as part of a journey to meet their God. However, they are strongly encouraged to seek all kinds of treatment Athar, 1998). "Illness is one of the forms of experience by which humans arrive at acknowledge of Allah" (AlGhazzali, 1970). Health and illness become part of life, and prayer remains the salvation in both health and in sickness. It is narrated that the Prophet (PBUH) said that: "The prayer of the sick person will never be rejected, until he recovers." Islam encourages people to search for treatment for their illnesses and this is not in contradiction with reliance on Allah for a cure. The Prophet Muhammad said that: "Seek treatment, because Allah did not create a sickness but has created a treatment for it except for old age"(Bukhari)".

In Islam, individuals are encouraged to have balance in their life, which includes work, pray and looking after family. People are encouraged to adopt healthy lifestyle by eating in moderation, and doing regular exercise to avoid obesity and other diseases. Islam restricts sex to the legal bounds of marriage (Ahmed, 1999). The Holy Qur'an prohibits the eating of pork or pork products, meat of dead animals, blood of any kind and drinking alcohol. The consumption of food in moderation and leading a healthy lifestyle are considered as religious obligations. The Prophet taught that "your body has rights over you" and said that "Ask Allah for forgiveness and well-being". Cleanliness has both a physical and spiritual dimension. Athar (1998) cited the Hadith of the Prophet Muhammad that 'cleanliness is considered half of the faith". So, a high standard of personal hygiene is required by all Muslim. 
It was expected that dialysis patients would have poor QOL in terms of physical, psychological and social health. Dialysis patients are exposed to major life stressors such as dietary restrictions, changing role in the society, sexual dysfunction and depression. They spend around 20 hours per week in the hospital to receive dialysis treatment. The dialysis access (fistula and central lines are disfiguring) makes them socially unattractive. Furthermore, in this study it was expected that good physical health and the degree of disability that normally follow the disease process and late complications impact negatively on the QOL of the respondents. Having another chronic illness in addition to kidney failure was expected to have a major impact on the QOL of both dialysis patients and people from the community as it might add extra stressors on their life. Furthermore, living with two or more chronic illnesses was expected to affect all dimensions of QOL because patients with two chronic illnesses have to live with their limitations and the complications of those chronic illnesses.

\subsubsection{Family}

From Islamic perspectives, the family is critical. "The family is the nucleus of the society; whenever the nucleus is healthy and strong, so will be the whole structure" (Shuraida, 2001 , p. 10). In Islam the main purpose of marriage is to establish a family and to have children so that the message of Islam continues (Shuraida). Both men and women have an equally important but different role in forming a family and bringing up children (Abdussalam, 2001). Prophet Muhammad's first wife, Khadija, is frequently looked at as an example for Muslim women. Khadija was a successful businesswoman, politician and spiritual authority, supporting her husband in all circumstances (Smith, 1999).

In Islamic communities, the first support system is the family. The family usually takes the sick person to the hospital and works closely with the healthcare team to provide the best treatment and support. It is expected that dialysis patients have a great deal of psychological and physical stressors that require family and social supports as well as physical and psychological adaptation to the changes in their role and ability to fully function in society. The availability of family members and other support systems will have a positive impact on the QOL of dialysis patients. Muslim communities have strong family ties and this is part of the Islamic teaching. It was expected therefore that Muslim dialysis 
patients would score high in the family subscale. Caution should be maintained when asking people any questions with regards to their sexual life. Single men and women are not supposed to have any kind of sexual relationship before marriage. Muslim people may feel offended regardless of whether they are married or not, if asked to display any information about their sex life unless there is a medical indication.

\subsubsection{Economic resources}

Healthy people are to be found in all ethnic groups, but some ethnic groups are more disadvantaged in health than others. For example, Maori people in New Zealand, Aborigines from Australia and people from South East Asia (Braveman, 2006). In the UAE there is a range of socioeconomic classes. It was expected that people from poor socioeconomic classes such as South East Asians would have poor health in general. Dialysis patients from South East Asia who live in UAE are disadvantaged and would be expected to be less healthy and have a poorer QOL compared with other dialysis patients from other ethnic group. Sufficient income for dialysis patients plays an important role in providing the basic needs such as food and transportation expenses to and from the hospital as well as buying the prescribed medication. Having sufficient income may impact on the overall satisfaction with life and consequently having a better quality of life.

Living in the UAE for a longer period of time may impact positively on the QOL. Usually the living standards in the UAE are high compared with other countries. One might presume that the longer people live in UAE, the better QOL they have. In fact it depends on whether they come from a disadvantaged group or not. In contrast, the longer people live away from their original countries the less QOL they may have due to the less family support they receive. UAE Nationals were expected to have better social and economic support compared with other ethnic groups because they receive free medical treatment and have several ways of getting financial support from the government. It was also expected that other dialysis patients who have poor social and financial status would have poor QOL scores. Furthermore, it was expected that dialysis patients would have a great deal of psychological and physical stressors that require family and social supports as well as physical and psychological adaptation to the changes in their life such as the need for travel to and from the dialysis unit three times a week. 


\subsubsection{Life events}

Having major life events such as the death of family members, marriage, accidents or diagnosis with chronic illnesses will have a major impact on a person's perception of their QOL. Dialysis patients are exposed to major life events that change almost everything in their life. It was expected that they would have poor QOL scores compared with people from the community. Dialysis patients are more prone to hospitalisation, doctor visits, uncertainty about the future and loss of control over life in general (Power, Duncan, \& Goodlad, 2009). Major negative events such as the death of family members or friends were expected to make their QOL much poorer.

Islam is a caring religion. Muslims are required to be merciful and compassionate to others. Due to the compassionate and merciful nature of Islam, it is expected to have a major impact on how Muslims perceive their QOL. Whilst Islam is clearly against alcoholism, and homosexual practices, it does not ban Muslim nurses and other healthcare professionals from caring for both Muslim and non-Muslim patients who engage in these activities. There are no differences in the provision of care for Muslim and non-Muslim patients. Hence, in healthcare people are treated equally.

In summary, this framework proposes that the interaction of several key factors contributes to the QOL of dialysis patients that shape the view of individuals, health, families, community: cultural values, customs, religious, life events, and economic resources. QOL is a cultural construct that is impacted upon by key life events. QOL is also an individual experience and perception that is shaped by cultural and societal values. QOL should not be viewed in isolation. While health remains the common aspect that is measured in almost all QOL tools, social, economic and psychological dimensions are also important aspects to measure. Incorporating these elements in this conceptual framework will enrich the nursing knowledge of the QOL of people from Muslim countries with different ethnic backgrounds.

\subsection{Conclusion}

A variety of QOL tools have been used in the literature to study QOL of dialysis patients. Each tool has different subscales. This research used the QOL Index Dialysis version for 
the dialysis sample and the QOL Index Generic version for general population sample and SF 36 for both samples. This study has used a general health-related QOL tool (SF-36) and a disease -specific (QOL Index). The SF-36 measures the ability to perform a task and the performance of tasks, impairment and disability. In contrast, the QOL Index measures the perception of QOL in terms of satisfaction with a certain aspect of life and the importance of those aspects to the individuals, such as satisfaction and importance of sexuality, spirituality, relationship, and self efficacy. Both tools were assessed against the selection criteria suggested by Mangione (2002). Researchers need to be aware of category fallacy and response editing when interpreting research findings. The conceptual framework was developed to guide data collection and interpretation, taking into consideration the cultural and religious background of the participants. The conceptual framework described the expected effect of culture, society, religion, physical health, family, economic resources, and life events on the QOL. It was expected that the UAE population would have a unique perception of their QOL; it might be influenced by their culture, previous experience, financial status, personal and country wide values, religion, and aspirations. Furthermore, it was also expected that dialysis patients in the UAE might give different meaning to the QOL concept compared with healthy individuals from the same country. The next chapter explores the methodology used in this research and explains the data collection and analysis. 


\section{Chapter 4 Methodology}

This chapter describes the methodology and methods used in this study. Section one states the research questions and aims. A full description of the research design is presented in section two. Section three describes the pilot study. Section four describes the sampling process and the recruitment of participants for the dialysis and community groups. The data collection section is described in section five. The steps taken to evaluate the cultural relevancy of the tools are described in section six. The ethical issues such as maintaining patients' confidentiality and obtaining informed consent are explained in section seven. Section eight describes the managements of data entry, missing data, survey participants, data distribution. The analysis of cultural relevancy of the tools and qualitative data were described in this section. It also describes the tests for establishing the correlations and differences of socio-demographic and life factors with QOL total scores and demographic differences and similarities between the dialysis and the community samples, investigation of the multiple regression analysis, and multivariate analysis of variances.

\subsection{Background}

Several research designs have been used to study QOL. The survey design was the commonest methodological approach used to study QOL in dialysis patients (Acaray \& Pinar, 2005; Coelho-Marques et al., 2006; Hicks et al., 2004; Merkus et al., 1997; Neto et al., 2000). Several qualitative approaches have also been used to study QOL. Rittman, Northsea, Hausauer, Green and Swanson (1993) used hermeneutical analysis to study the experience of patients living with chronic renal failure within the framework of Heideggerian phenomenology. Moreover, Hagren, Pettersen, Severinsson, Lutzen and Clyne (2001) used a qualitative interpretative content analysis to describe patients' experiences of suffering from kidney failure. Polaschek (2003) used a critical interpretive approach to study the experience of a group of Caucasian men with kidney failure managing their own haemodialysis therapy in their homes.

\subsubsection{Research questions and aims}

This research studied the factors that influenced QOL and highlighted the differences in QOL between dialysis patients and the community sample from UAE. Moreover, this 
research examined whether the QOL tools used in this research were culturally relevant and which QOL tool was more culturally relevant. Furthermore, it explored the impact of gender, ethnicity, and other demographic variables on the QOL of these two samples. Lastly, the impact of dialysis adequacy and nutritional status on the $\mathrm{QOL}$ of dialysis patients were also investigated.

The aims of this study were to:

1- Establish what is important in respect to the QOL for people living in the UAE

2- Compare the cultural relevancy of two QOL tools between dialysis patients with a sample of the community living in UAE

3- Study the differences of QOL between dialysis patients in UAE and a sample of the general population

4- Identify the physical, psychological, social, cultural, religious, gender, ethnicity influences and impacts on the QOL of people undergoing dialysis and a sample of the general population

5- Examine the impact of dialysis adequacy and nutritional status on the QOL of dialysis patients.

\subsection{Research design}

This research used a descriptive survey design. In this study, the Strengthening the Reporting of Observational Studies in Epidemiology (STROBE) statement, developed by von Elm et al. (2007) for the STROBE initiative, was used as a guide for managing and reporting the methodology, data collection and findings. The descriptive survey design was chosen for the following reasons. Firstly, it is practical. Secondly, participants can assess their own QOL using well-structured tools; this allows them to reflect the true image about their QOL. Thirdly, involving patients in data collection and assessment of their own QOL using tools with proven reliability and validity gives participants a sense of involvement and partnership. Lastly, this design was considered the best suited to the culture of people living in the UAE because it will allows participants to answer the tools in their own privacy in their own time. 
Descriptive research designs result in a description of the data in forms such as words, pictures, charts and tables. The type of description depends on how much information the researcher knows about the topic before data collection. Level I questions are useful when little or no information is known about the topic, and leads to exploratory descriptive designs. Level II questions, arise when the variables are known but their action cannot be predicted and leads to descriptive comparative survey designs (Polit \& Beck, 2004). In this study, the variables that may predict or contribute to the QOL were presented in the conceptual framework, but their action or effect on the UAE population is not known. Therefore, this research used a quantitative approach with both exploratory and comparative descriptive survey designs to examine the QOL in dialysis and community samples using two QOL tools at two stages. The first stage examined the cultural relevancy of these tools for participants. The second stage explored the QOL of the selected participants using two internationally validated QOL tools. To study the effect of clinical variables on the QOL of dialysis patients, data were collected on serum creatinine, albumin and haemoglobin levels and urea reduction ratio (to measure dialysis adequacy and nutritional status).

The decision to use two QOL tools in this research was informed by the gaps in the literature. Few studies have been found in the literature that used two QOL tools to compare the QOL in dialysis patients (Huang et al., 2006; Neto et al., 2000). Using two QOL tools allows a comparison between them using the same population and provides a chance to examine comprehensive information about the impacts on QOL of the respondents. Furthermore, it provides the opportunity to study more dimensions of the QOL. The SF-36 is a general tool while the QOL Index dialysis version is a diseasespecific tool. The SF-36 was primarily designed to measure health and or health-related QOL. The SF-36 measures the ability to perform a task as well as assess the degree of impairment and disability. In contrast, the QOL Index measures the perception of the QOL in terms of satisfaction with certain aspect of life and the importance of those aspects to the individuals such as satisfaction and importance of sexuality, spirituality, relationships, and self-efficacy. Therefore, it has the benefit of measuring how functional change affects life rather than just measuring functional change. 


\subsubsection{Advantages and disadvantages of descriptive survey design}

Descriptive survey design sometimes involves distributing unsupervised self-administered questionnaires (Ferrans \& Powers, 1992). Advantages of this design include low cost and the ability to reach large numbers of people across a wide geographic area. Also, it allows respondents to respond in their own time (Oppenheim, 2001). On the other hand, the disadvantages of descriptive survey design are sample related issues such as the need to must ensure that a large number of the selected sample will reply, availability of the mailing list, representation of the sample, variation of response rate, literacy levels and language difficulties (Oppenheim). Some respondents may choose the most desirable and socially acceptable responses. The design of the questions was chosen to be simple and easy to understand. Therefore, it was expected that participants will not have any difficulties in reading and understanding the questions.

\subsection{Piloting the survey}

After the ethics approvals from Victoria University of Wellington Human Ethics Committee and the SKMC Ethics Committee (Appendix $1 \& 2$ ), the tools were pre-tested with four hemodialysis patients and four healthy people (two male and two female) from the general population. All eight people were asked to answer the total survey package and comment on any questions they felt should not be included or could be modified. Positive responses were received from all eight people and minor modifications to the demographic data items were made in response to the suggestions. These modifications included changes to the items on living arrangements, by adding a living with friends' option, and to the items on educational levels by adding a completed secondary school certificate option.

\subsection{Sampling}

This section describes the sampling process for the dialysis and the community samples.

\subsubsection{Dialysis sample and recruitment of participants}

Randomised selection process is the ideal technique for determining the generalizability of survey findings (Barnett, 1991; Burns \& Grove, 2003). However, this was not possible in this research because the number of dialysis patients at SKMC was relatively small. The researcher therefore aimed to recruit all hemodialysis patients at SKMC. The total number 
of dialysis patients was 192. Prior to data collection the researcher aimed for having easy access to the selected sample, lower costs, less chance in missing the right participants and better returns of the distributed questionnaires. However, given the variability of dialysis services across the UAE that was described in Chapter 1, the sample in this study is not representative of all dialysis patients in UAE. The researcher was aware at the outset of the study that a limitation of choosing a sample from only one hospital would mean that the results might not be generalizable to all dialysis patients in UAE. Furthermore, no power calculation was undertaken to determine the required sample sizes as there were no UAE normative values on which to base such an analysis.

Three inclusion criteria were used to ensure accurate data collection from participants. The first criteria restricted the participation in the study to dialysis patients who had been undergoing regular hemodialysis therapy for more than three months, to omit the influence of metabolic factors in the early stage of haemodialysis on consciousness level such as uremic encephalopathy. The second criteria required that patients should not have apparent cerebro-vascular disease or serious intellectual impairment, to avoid missinterpretation of the questions. Patients' files were reviewed for previous or current episodes of cerebro-vascular disease before the distribution of the survey packages. The third criteria was participants should be 18 years or over, to enable informed consent at legal age for the UAE.

\subsubsection{Community sample and recruitment of participants}

The researcher approached 400 people at random. The people were from different organisations and shopping centres in Abu Dhabi. Participants were asked if they were interested in participating in the study and if they were, they were given the survey package and asked to return the completed survey by post within two days and return it to the researcher by post. Inclusion criteria for the community sample were similar to the dialysis sample except they were not on dialysis. It was expected that participants would have the ability to complete the survey package in the Arabic or English language.

From the outset of this research, it was known that it would be difficult to obtain a representative sample from all cities in the UAE. To have a representative sample from the community the researcher considered selecting friends or family members of people on 
dialysis. However, the literature review revealed that the QOL of caregivers and family members of patients with chronic illnesses were highly affected (Palmer, 2003), so this option was rejected.

\subsection{Data collection}

Data collection for this research was conducted by the researcher between May and July 2007. An independent nurse approached the potential participants and provided them with the invitation letter (Appendix 3). The invitation letter described the purpose of the research and highlighted the importance of responding within the specified period. Moreover, the invitation letter reassured participants that confidentiality would be maintained. Once they indicated their willingness to be involved in the study, they were asked to sign a consent form (Appendix 4). Consenting patients were given the survey package containing the demographic survey that included the three open-ended questions, QOL Index dialysis version, SF-36 (Appendix 5) and an addressed envelope for the return of the surveys. Patients had the choice of completing the survey package in Arabic or English. For both samples, Arabic speaking participants were given the Arabic versions of the tools and English speaking participants were given the English versions of the tools. Participants who were fluent in both languages were given the choice of completing the survey package in either Arabic or English. Then participants completed the survey on their own. Participants who wanted to participate but who had problems in reading or understanding the questionnaires or could not speak and write English or Arabic, were assisted by hemodialysis nurses. The nurses translated the questions into the participant language and filled in the sheet with the participant's responses. The researcher is aware that this is a limitation of data collection, but few patients needed help in completing the survey package. The effect of educational level was one of the variables that were included in the study.

For the community sample, participants were given the invitation letter (Appendix 6). If they chose to be involved in the study, they were asked to sign the consent form (Appendix 7) then asked to complete the survey package within two days, which included demographic data and three open-ended questions, SF-36 and the generic version of QOL Index (Appendix 8). An envelope was provided for the return of the survey package. The 
researcher approached 400 potential candidates of which 350 participants agreed to participate in the survey.

To increase the return rates of the distributed surveys the following measures were taken. An envelope to return the questionnaires was provided as per the suggestion of Edwards et al. (2002). Furthermore, participants were asked personally by the recruiting nurse or the researcher if they were interested in participating in the study before being given survey package sending the questionnaires. The information sheet was designed to be of interest to participants.

\subsection{Cultural relevancy of the tools}

The methods to establish the cultural relevance of the tools needed to let respondents decide which QOL tools were most relevant and whether there were any particular items in the tools that were culturally irrelevant. Such an approach assigns importance to the individual's subjective appraisal of what is important for an individual ir relation to their health status and other life circumstances. It presumes that QOL is at least partly independent of health status (Guyatt, Feeny, \& Patrick, 1993), and reflects the way people views and respond to their health status and to other aspects of their lives (Gill \& Feinstein, 1994).

To establish the cultural relevancy of the tools and to identify which tool was more culturally relevant to people live in the UAE, this research used four steps. Firstly, the cultural relevancy of each tool was studied by asking three questions. The first question asked respondents to state whether they considered that the SF-36 was relevant to their culture and what needed to be added or deleted from the tools to make them culturally relevant. The second question asked respondents to state whether they considered that the QOL Index was relevant to their culture. The third question asked respondents to state which tool they judged to be more culturally relevant. Secondly, this research examined the missing data to explore whether respondents missed answering questions due to evidence of non applicability or due to cultural irrelevancy. Thirdly, this research studied and compared the QOL between the dialysis patients and a sample from the community. Fourthly, three open-end questions were asked to capture what was important to 
respondents regarding QOL. The first question requested respondents to state three things that the people living in UAE valued most in life, to explore how people in the community perceive or understood the QOL. The second question requested respondents to state three things they personally valued most in life, to examine individual perception of the QOL. The third question requested respondents to think of three things that were most important to them in maintaining or improving their QOL.

\subsection{Ethical issues}

Prior to commencement of this study, approvals were obtained from the Victoria University of Wellington Human Ethics Committee and SKMC Ethics Committee (See Appendices 7 and 8 for approval letters). Also, approvals to use the complete research tools were granted from their authors. Participants were instructed in the consent form (Appendix 2) that they could decline to answer any questions they were not comfortable with. Dialysis participants were asked to sign the consent form after they received the full description of the study from the recruiting nurse. Confidentiality issues were highlighted in both the invitation letter (Appendix 1) and the consent form (Appendix 2). Dialysis patients were given the choice to take the survey package home or to fill it in while at the dialysis unit. Patients were given full privacy in the dialysis unit when answering the survey questions and were reassured that confidentiality would be maintained. They were instructed in the consent form not to include their names or hospital numbers or any mark that might identify them. The researcher's job at that time was clinical resource nurse which involved educating both nursing staff and dialysis patients about dialysis treatment. The researcher did not have any influence on the selected sample or intervene in any way with the participants who completed the survey. Patients were also reassured that their care would not be compromised if they chose not to participate in the study.

The concepts from medical and nursing ethics of autonomy, beneficence, dignity and respect, informed consent and confidentiality underpinned this research (Oman, Krugman, \& Fink, 2003). Autonomy was addressed by giving all potential participants a choice about participating in the study. Patients were reassured that their care would not be compromised if they chose not to participate. Furthermore patients who agreed to participate were given the choice to take the survey package home to answer it at their 
convenience. The fact that some participants took the forms and did not complete them or did not answer all the questions is an indication that autonomy was maintained. The concept of beneficence requires that the researcher act in the best interest of the patient when conducting research. This concept informed this research, as the goal was to improve the quality of care for dialysis patients. The concepts of dignity and respect were addressed in how people were approached to participate in the study and in the design decisions about the cultural relevancy of the tools. Participants were advised they did not have to answer any questions that they were not comfortable answering, and were also provided an opportunity to comment on the tools and quality of life generally. All answers provided in the open-ended questions were included in the analysis. The concepts of informed consent and confidentiality were demonstrated by providing information sheets in two languages with the details of the study and by asking people not to put any names on the returned documentation.

\subsection{Data analysis}

This section describes data entry, cleaning and missing data. It also explains the phases of data analysis.

\subsubsection{Establishing and preparing the data base (data entry)}

Data were analysed using the SPSS software for Windows version 13.0 (SPSS Inc, Chicago, IL, U.S.A.). Prior to entering data into the SPSS software, a coding sheet was developed to ensure data from each of the survey groups was entered consistently. The coding sheet was kept to document this and to note any modifications undertaken during analysis. The coding sheet was saved in computer and paper format. To avoid mistakes during data entry, data were initially coded with a single easily recognisable symbol. Nominal variables were given either a letter or a number such as $M$ for male and $F$ for female. Interval variables were entered as numbers. This sometimes involved converting the data returned into a different format. For example, the time living in UAE, and the last time participants travelled outside of the UAE were all converted from years to months as there were some patients who lived in or travelled out of UAE in less than one year. Dialysis patients were asked to state how long they have been on dialysis in years and months, and this was converted to months to facilitate data analysis. 
After data entry, the data were checked for accuracy by doing frequency testing. This checking process was to ensure that each variable type (nominal, ordinal, scale) was correct. Two ways for data cleaning were used. First, to ensure correct code, variables were each checked to see whether there were any inaccurate codes or values. For example, this research only involved adults; if the age range data had a number less than 18 this would indicate a wrong entry. Second, for checking the categorical variables for errors, the main SPSS menu was used to generate descriptive statistics as per steps outlined in Pallant (2007). Few errors were found during this cleaning process. If errors were found, the researcher checked the original questionnaire and corrected accordingly.

\subsubsection{Missing data}

Descriptive statistics were done to find out the percentages of the missing data for each variable and to explore whether the missing data happened randomly or in a systematic pattern. Randomly missed data would indicate unintentional errors made by respondents, while systematic patterns on missing data could indicate that respondents did not feel comfortable answering those questions or the missed questions were not applicable to them. Missing data for each of the QOL tools was addressed as per the tools' authors' guidelines. For the SF-36, this involved individual calculations of missing scores within the scale then using the scoring program to generate subscale and total scores (Quality Metric Scoring Program, 2007). The exclude cases pair wise option was used; this option normally excludes the case only if they are missing the data required for a specific analysis, but includes those for which they have the necessary information (Pallant, 2007). For the QOL Index, there was no need to replace missing data for items as scores were calculated based on the items answered for each person. For the SPSS scoring program, zero was not entered for missing data because the SPSS programme would consider it as a response and therefore produce incorrect scores. Analyses of missing data were undertaken as part of examining tools for cultural relevancy.

\subsubsection{Description of the survey respondents}

This stage involved developing a detailed description of the socio-demographics of the survey respondents for each group, and determining the data distribution to inform further analyses. Descriptive statistics such as frequencies presented as percentages, mean, 
range and standard deviation were used. Following this descriptive analysis, several variables were regrouped to enable the planned regression analyses to be completed. Given the sample size, regression analysis required the answer options to be regrouped to have natural alignment and have fewer answers. For example, the marital status variable was regrouped into single, married and others. The education variable was regrouped into 'did not attend school', 'primary school', 'secondary school' and 'tertiary education'. The religion variable was regrouped into 'Muslims', 'Christians' and 'others'. The living arrangement variable was regrouped into 'live alone', 'live with family' and 'others'. The employment variable was regrouped into 'full time employed', 'housekeeper / student / part time employed/others', 'retired / disabled' and 'unemployed'.

\subsubsection{Blood results of the dialysis sample}

The blood results obtained for the study were those that are routinely collected on a monthly basis before a patient commences dialysis. The results report on patients' dialysis adequacy (urea reduction ratio) and nutritional status (haemoglobin level, albumin, urea, and creatinine levels). The blood results were recorded by the hemodialysis nurses at the time of giving the survey package. Haemoglobin was used to assess the patient's degree of anaemia, with target minimum values of $11 \mathrm{~g} / \mathrm{dL}$ as per NKF-K/DOQI guidelines (2006). Serum albumin, urea and creatinine were used as a marker of nutritional status as per Chertow (1999). The target established albumin level in this study was $36 \mathrm{~g} / \mathrm{dL}$. The adequacy of the dialysis dose was measured using the urea reduction ratio, employing the mono-compartmental model of variable volume. The target established in this study was a minimum of $65 \%$ as per NKF-K/DOQI guidelines (2006).

\subsubsection{Data distribution}

Five statistical tests were performed to establish the normal distribution of the data. Firstly, the differences between the mean and the median were used. Minimal differences between the mean and the median would suggest a normal distribution of the data. Secondly, the Kolmogorov-Smirnov $Z(K S)$ test results of $<0.05$ would indicate that the data were not normally distributed (Peat \& Barton, 2006). Thirdly, the distribution of the variables was obtained from the Skewness and Kurtosis statistics. The values above +3 and -3 would indicate that the data were not normally distributed (Peat \& Barton). Fourthly, normality plots in the form of histograms show the frequency of measurements and the 
shape of the data and therefore provide a visual judgment of whether the distribution approximates to a bell shape. Also, the histograms show whether there were any gaps in the data, whether there were any outlying values and how far any outlying values were from the reminder of the data (Peat, \& Barton). Fifthly, the normal Q-Q plot is used to show each data value plotted against the value that would be expected if the data came from normal distribution. If the variable was normally distributed, the points would fall directly on the straight line. Any deviation from the straight line would indicate some degree of nonnormality (Peat, \& Barton).

\subsubsection{Analysis of QOL tools}

The total scores and the subscales scores of each tool were calculated using SPSS software. For each group, the total scores of each tool were calculated as per the guidelines from the tools authors'. Independent variables were printed using descriptive statistics such as mean, standard deviation, median, mean and inter-quartile range (IQR). The total scores of the QOL Index were converted to percentages for comparative purposes with the SF-36 scores. The subscales of the tools were analysed to find out the difference in each dimension between the dialysis patients and community sample. The researcher compared dialysis patients total scores and subscales scores with the community sample to find out if dialysis patients assessed QOL differently.

\subsubsection{Qualitative data}

The three open ended questions were used to capture the issues related to QOL that may not be captured by the tools. Participants were given the freedom to describe what they think other people in the UAE society value most in life, that they personally value, and what are the factors that may improve and maintain their QOL. Descriptive research can also be used to describe categories of qualitative information (Pope, Ziebland, \& Mays, 2002). The QOL tools used were developed from a Western perspective and therefore may reflect a Western background, encapsulating a western way of thinking and living. Using these tools for participants from other cultural backgrounds may not reflect the participants' actual perceived QOL. The decision to use descriptive survey designs and to compare two QOL tools and ask people some open-ended questions was informed by the literature review, and conceptual framework presented in the preceding two chapters. 


\subsubsection{Qualitative data: Analysis and ranking of the themes}

To analyse the answers from the open-ended questions, a content analysis method was used. This involved analysing responses from each population separately and then comparing and contrasting the findings. In content analysis, there is immersion in the text, by using more structured analytical techniques that involve sorting, categorising, naming themes and counting (Hsieh \& Shannon, 2005). Sorting responses involved entering responses into Microsoft Excel spread sheet as key words. Data were then transferred into SPSS data sheet to categorise the responses. Responses were entered under variables headings. Once all the responses to the first question were entered, responses that had similar meaning were re-coded in to similar variables. Then variables that had a low number of respondents were re-coded into other variables that carried similar themes. Content analysis started with keyword frequencies. Words and phrases mentioned most often reflected important concerns. The themes were ranked according to the number and percentages of the respondents who chose them.

\subsubsection{Correlations and differences of socio-demographic and life factors with QOL total scores}

The impact of the demographic variables on the total scores of the SF-36 and QOL Index and their subscales were explored. To explore the data in more depth, descriptive statistics that included mean and standard deviation were used. The correlation co-efficient between the total scores of the tools and the demographic variables was evaluated. According to Pallant (2007) correlation scores of 0.00-0.19 indicate slight or almost negligible correlation, 0.20-0.39 indicates low or quite small correlation, 0.40-0.69 indicate moderate correlation, $0.70-0.89$ indicate high correlation and scores of 0.90-1.00 indicate very high correlation. This phase of the analysis also involved a series of statistical tests to establish which social and demographic variables correlated with or had an impact on the SF-36 and QOL Index total scores. These tests were done separately for each population dataset. Then a comparison was made between demographic variables and the tools total scores.

\subsubsection{Demographic differences and similarities between the dialysis and the community samples}

A comparison of the demographic variables (predictor variables) between the dialysis and the community samples using descriptive statistics such as percentage, mean and 
standard deviation was done to establish the correlation between the predictors and the outcome variables in both samples. Then a comparison of the total scores of the SF-36 and QOL Index between the dialysis and the community samples was done to compare the findings. A series of statistical tests was done to establish what social-demographic variables correlated with or had an impact on the SF-36 and QOL Index total scores. Pearson's correlations were used for continuous variables, Spearman's correlation for ordinal data, and one-way analysis of variance (ANOVA) for nominal variables. To establish the cultural relevancy of the QOL tools, participants answered a group of questions. Answers were compared for numbers and percentages. Also, to establish the statistical significant differences in the responses to the cultural relevancy questions, $t$ test was used.

\subsubsection{Multiple regression analysis}

Under the direction and guidance of a statistician a multiple linear standard regression analysis was performed to determine variables that might affect the QOL scores among dialysis patients and the community samples. Initially standard multiple regression analysis was used to assess the ability of the predictor variables to predict levels of total scores of each of the QOL tools (Pallant, 2007). Moreover, the regression analysis was done separately for each sample to analyse the associations between independent variables (predictor variables) and QOL total scores (outcome variables).

For the dialysis sample, the multiple regression analysis was done at three levels. Level one included running a multiple regression analysis of SF-36 and the QOL Index total scores with demographic variables. Level two included running a multiple regression analysis of the SF-36 and the QOL Index total scores with the categorical variable using dummy variables approach. Level three, included running a multiple regression analysis of SF-36 and QOL Index total scores with the clinical laboratory variables. Then, another model was generated for each sample to determine how much of the total variability in the SF-36 and the QOL Index was predicted by all the variables that included all the continuous variables, all the dummy variables and the clinical variables (Pallant, 2007).

For the community sample, the multiple regression analysis was done at two levels. Level one included running a multiple regression analysis of the SF-36 and the QOL Index total 
scores with demographic variables. Level two included running a multiple regression analysis of SF-36 and QOL Index total scores with the categorical variable using dummy variables approach. Then, another model was generated to determine how much of the total variability in SF-36 and QOL Index was predicted by all the variables that included all the continuous variables and all the dummy variables.

The regression coefficient (Beta values) and their significance are important statistics to consider. Beta values give an indication of the contribution of each independent variable in explaining the dependent variables. Positive values represent positive relationship between the predictor variables and the outcome variable and negative values represent a negative relationship. Also, the greater the Beta values under standardised coefficient indicate strongest contribution in explaining the dependent variable regardless of the negative or positive signs. The significant value indicates whether a variable has made a statistically significant contribution to the equation. If the significant value is less than 0.05 , the variable is making a statistically significant contribution to the prediction of the dependent variable. $R^{2}$ values are the values of multiple correlation coefficients between the predictors and the outcome. The $\mathrm{R}^{2}$ values measure how much of the variability in the outcome is accounted for by the predictor expressed in percentage (Pallant, 2007).

\subsubsection{Multivariate analysis of variances (MANOVA)}

The MANOVA analysis adjusts for the demographic differences in both samples. Given the relatively small sample size of the study, the MANOVA could only be done on total scores. of the tools. The multivariate analysis of variances analysis was done for each sample separately. All variables were included in the analysis. The dependent variables for both samples were the total scores of the SF-36 and the total scores of the QOL Index tools. The fixed factors for the dialysis sample were gender, ethnicity, marital status, religion, living arrangement, employment, education, life event, chronic illness and cause of kidney failure variables. The covariates for the dialysis sample were age, living in UAE, travel out of UAE, year on dialysis, cause of kidney failure, haemoglobin, serum albumin, pre-dialysis Blood Urea Nitrogen (BUN), pre-dialysis creatinine, and URR. For the community sample, the fixed factors were gender, ethnicity, marital status, religion, living arrangement, employment, education, life event and chronic illness variables. The covariates for the community sample were age, living in UAE and travel out of UAE variables. 
The findings are reported as statistically significant when $p$ value is less than or equal to 0.05 ( $p \leq 0.05$ ). The effect size of the relationship was assessed by Partial Eta Squared value. It ranges between 0 and 1. If the Partial Eta Squared value from 0.00 to 0.01 the relationship considered week or small. If the Partial Eta Squared value from 0.02 to 0.06 the relationship is considered medium and if the Partial Eta Squared value from 0.07 to 0.14 or above the relationship is considered large (Pierce, Block, \& Aguinis, 2004). Summary of the quantitative data analysis is presented in Tables 4.1, 4.2, and 4.3. These tables reflect the order in which the analysis was undertaken.

\section{Table 4.1 Descriptive Statistical methods}

\begin{tabular}{|l|l|}
\hline Frequency and percentages used to find out the: & $\begin{array}{l}\text { Mean and Standard Deviation M(SD) used } \\
\text { to find out the: }\end{array}$ \\
\hline $\begin{array}{l}\text { Comparison between the cultural relevancies of both } \\
\text { tools }\end{array}$ & $\begin{array}{l}\text { Comparison of continuous socio- } \\
\text { demographic factors for both samples }\end{array}$ \\
\hline $\begin{array}{l}\text { Demographic characteristics of the respondents who } \\
\text { considered QOL tools were not culturally relevant by } \\
\text { sample. }\end{array}$ & $\begin{array}{l}\text { Comparison between the SF-36 subscales } \\
\text { and the total scores of both samples. }\end{array}$ \\
\hline Dialysis respondents' opinion on the QOL tools. & $\begin{array}{l}\text { Comparison between subscales and total } \\
\text { scores of QOL Index of both samples. }\end{array}$ \\
\hline Missing data of the open-ended questions. & $\begin{array}{l}\text { Comparison of the findings between the } \\
\text { demographic variables and SF-36 total } \\
\text { scores in both samples. }\end{array}$ \\
\hline $\begin{array}{l}\text { Responses of open-ended questions for both } \\
\text { samples. }\end{array}$ & $\begin{array}{l}\text { Comparison of the findings between the } \\
\text { demographic variables and QOL Index total } \\
\text { scores for both samples. }\end{array}$ \\
\hline $\begin{array}{l}\text { The characteristics of respondents who missed } \\
\text { questions on the SF-36 and QOL Index tools }\end{array}$ & Mean of blood results of the dialysis sample \\
\hline $\begin{array}{l}\text { Questions that had highest missing responses in the } \\
\text { QOL Index tool }\end{array}$ & \\
\hline
\end{tabular}


Table 4.2 Inferential Statistics - Bivariate Analysis

\begin{tabular}{|c|c|c|c|}
\hline $\begin{array}{l}t \text { - test used for the } \\
\text { Comparison of the } \\
\text { proportion } \\
\text { (percentages) } \\
\text { between the } \\
\text { demographics of } \\
\text { both samples }\end{array}$ & $\begin{array}{l}\text { t- test used for the } \\
\text { Comparison the mean } \\
\text { of the two samples. }\end{array}$ & $\begin{array}{l}\text { Analysis Of } \\
\text { Variance (ANOVA) } \\
\text { used to }\end{array}$ & $\begin{array}{l}\text { Pearson Correlation } \\
\text { Coefficient to find out } \\
\text { the }\end{array}$ \\
\hline \multirow[t]{5}{*}{$\begin{array}{l}\text { Comparison of } \\
\text { continuous socio- } \\
\text { demographic } \\
\text { factors for both } \\
\text { samples. }\end{array}$} & $\begin{array}{l}\text { Comparison of continuous } \\
\text { socio-demographic } \\
\text { factors for both samples. }\end{array}$ & $\begin{array}{l}\text { Comparison of the } \\
\text { findings between } \\
\text { the demographic } \\
\text { variables and SF-36 } \\
\text { total scores in both } \\
\text { samples. }\end{array}$ & $\begin{array}{l}\text { Correlation between } \\
\text { demographic data and } \\
\text { SF-36 total scores. }\end{array}$ \\
\hline & $\begin{array}{l}\text { Comparison between the } \\
\text { SF- } 36 \text { subscales and the } \\
\text { total scores of both } \\
\text { samples. }\end{array}$ & $\begin{array}{l}\text { Comparison of the } \\
\text { findings between } \\
\text { the demographic } \\
\text { variables and QOL } \\
\text { Index total scores } \\
\text { for both sample. }\end{array}$ & $\begin{array}{l}\text { Clinical variables } \\
\text { correlations with SF-36 } \\
\text { total scores for the } \\
\text { dialysis sample. }\end{array}$ \\
\hline & $\begin{array}{l}\text { Comparison between } \\
\text { subscales and total } \\
\text { scores of QOL Index of } \\
\text { both samples }\end{array}$ & & $\begin{array}{l}\text { Comparison of the } \\
\text { findings between the } \\
\text { demographic variables } \\
\text { and QOL Index total } \\
\text { scores for both samples. }\end{array}$ \\
\hline & $\begin{array}{l}\text { Comparison of the } \\
\text { findings between the } \\
\text { demographic variables } \\
\text { and SF-36 total scores in } \\
\text { both samples }\end{array}$ & & $\begin{array}{l}\text { Correlation between } \\
\text { demographic data and } \\
\text { QOL Index total scores. }\end{array}$ \\
\hline & $\begin{array}{l}\text { Comparison of the } \\
\text { findings between the } \\
\text { demographic variables } \\
\text { and QOL Index total } \\
\text { scores for both samples }\end{array}$ & & $\begin{array}{l}\text { Lab values correlations } \\
\text { with total scores QOL } \\
\text { Index. }\end{array}$ \\
\hline
\end{tabular}

Table 4.3 Inferential Statistics - Multivariate Analysis

\begin{tabular}{|l|l|}
\hline \multicolumn{1}{|c|}{ Multiple Regression Analysis } & \multicolumn{1}{c|}{ MANOVA tests } \\
\hline $\begin{array}{l}\text { Multiple regression analysis of SF-36 and QOL Index total } \\
\text { scores with demographic variables for dialysis sample. }\end{array}$ & $\begin{array}{l}\text { MANOVA tests for dialysis } \\
\text { sample }\end{array}$ \\
\hline $\begin{array}{l}\text { Multiple regression analysis of SF-36 and QOL Index total } \\
\text { scores with dummy categorical variables for dialysis sample. }\end{array}$ & $\begin{array}{l}\text { MANOVA tests for the } \\
\text { community sample }\end{array}$ \\
\hline $\begin{array}{l}\text { Multiple regression analysis of SF-36 and QOL Index total } \\
\text { scores with the clinical variables for dialysis sample }\end{array}$ & \\
\hline $\begin{array}{l}\text { Multiple regression analysis of SF-36 and QOL Index total } \\
\text { scores with demographic variables for community sample }\end{array}$ & \\
\hline $\begin{array}{l}\text { Multiple regression analysis of SF-36 and QOL Index total } \\
\text { scores with dummy variables for community sample Table. }\end{array}$ & \\
\hline
\end{tabular}


The next chapter reports on the findings of the cultural relevancy of the QOL tools. 


\section{Chapter 5 Findings related to the samples and the cultural relevancy}

This chapter is the first of two chapters that report the findings of the study. This chapter describes the samples of people who participated in the study and the findings related to cultural relevancy of the tools. Section one provides details related to the return rate. The comparison between the demographic and the the continuous socio-demographic variables for both samples are presented in section two. The level of missing data for both samples and a description of the characteristics of respondents who missed questions from both tools are presented in section three. Section four reports on the tests used to examine data distribution. The cultural relevancy of both tools for the two samples is reported in section five. Section six reports the findings from the qualitative data. It also compare the responses to open-ended questions for both samples. This section also reports on the themes that were identified by the respondents. Chapter 6 presents the discussion of the findings from chapter five.

The results were reported using standard statistical symbols. These symbols are listed in Table 5.1.

Table 5.1 Statistical symbols and abbreviations

\begin{tabular}{|l|l|}
\hline Symbols & \multicolumn{1}{|c|}{ Abbreviations } \\
\hline$\%$ & Percentage \\
\hline $\mathrm{N}$ & Number \\
\hline $\mathrm{M}$ & Mean \\
\hline \pm & Standard deviation value \\
\hline IQR & Inter-quartile range \\
\hline $\mathrm{P}$ & Significant test \\
\hline $\mathrm{B}$ & Un-standardised coefficient \\
\hline $\mathrm{B}$ & Standard coefficient \\
\hline $\mathrm{R}$ & Correlation coefficient \\
\hline $\mathrm{Df}$ & Degree of freedom \\
\hline VIF & Variance Inflation Factor \\
\hline
\end{tabular}

To be consistent in reporting the results of this research, the findings are reported as statistically significant when the $p$ value is less than or equal to 0.05 and the findings are 
reported as having trends toward being statistically significant if the $p$ value is less than or equal to 0.10 .

\subsection{Return rates}

There were 192 dialysis patients at SKMC at the time of data collection. Thirty-one patients did not meet the inclusion criteria; 20 had been on dialysis for less than three months, seven had a cerebro-vascular accident in the past two years as per medical records and four patients were under the age of 18 years. The remaining 161 patients signed the consent form and received the survey package during their regular dialysis schedule days. The survey packages were distributed during May - July 2007. Two patients declined to participate after they looked at the survey; and five did not return the survey packages. Of the 154 returned survey packages, four were blank. The sample therefore consisted of 150 respondents which is equivalent to a $93 \%$ return rate. There were no demographic or clinical details available about the patients, who declined, did not return the surveys or returned incomplete surveys. In the community sample, 400 people were approached to participate in the study and 350 initially agreed to participate. Of the 350 respondents who received the survey packages, 276 survey packages were returned, of which seven were incomplete, and two were blank. The sample therefore consisted of 267 respondents which is equivalent to a $76 \%$ return rate. Two thirds of the survey packages were completed in Arabic and one third in English. Given that the survey was anonymous, no further analysis was done on the demographic characteristics of the respondents who completed the Arabic and the English versions. No demographic data were available on those who did not return the surveys or returned incomplete surveys. Ten in the dialysis sample were unable to read or write in English or Arabic and the assigned nurses translated the questions and recorded the answers for them.

\subsection{Demographic and clinical variables of both samples}

This section reports on the description of the demographic variables for both samples. The demographic differences and similarities of both samples are summarised in Table 5.2. 
Table 5.2 Comparison of the demographics of the dialysis and community samples

\begin{tabular}{|l|l|r|r|r|}
\hline Variable & & $\begin{array}{r}\text { Dialysis } \\
\mathbf{n}=\mathbf{1 5 0}(\mathbf{1 0 0 \%})\end{array}$ & $\begin{array}{c}\text { Community } \\
\mathbf{n = 2 6 7 ( 1 0 0 \% )}\end{array}$ & $\mathbf{p}$ value \\
\hline Gender & Female & $44(29.3)$ & $115(43.1)$ & $\mathbf{0 . 0 0 5}$ \\
& Male & $106(70.7)$ & $152(56.9)$ & \\
\hline Ethnicity & UAE National & $42(28.0)$ & $32(12.0)$ & $<\mathbf{0 . 0 0 0 1}$ \\
& Arab National & $57(38.0)$ & $136(50.9)$ & \\
& South Asian & $34(22.7)$ & $63(23.6)$ & \\
& Other & $17(11.3)$ & $36(13.5)$ & \\
\hline Marital status & Single & $15(10.0)$ & $56(21.0)$ & $<\mathbf{0 . 0 0 0 1}$ \\
& Married & $113(75.3)$ & $199(74.5)$ & \\
& Divorced or widowed & $22(14.7)$ & $12(4.5)$ & \\
\hline Religion & Muslim & $123(82.0)$ & $180(67.4)$ & $<\mathbf{0 . 0 0 0 1}$ \\
& Christian & $16(10.7)$ & $72(27.0)$ & \\
& Others & $11(7.3)$ & $15(5.6)$ & \\
\hline Living & Lives with family & $100(66.7)$ & $196(73.4)$ & $\mathbf{0 . 0 4 9}$ \\
arrangement & Lives alone & $12(8.0)$ & $29(10.9)$ & \\
& Other & $38(25.3)$ & $42(15.7)$ & \\
\hline Employment & Full time employment & $56(37.3)$ & $213(79.8)$ & $<\mathbf{0 . 0 0 0 1}$ \\
& Housekeeper, student, part & $44(29.3)$ & $38(14.2)$ & \\
& time employed & & & \\
& Retired and disabled & $24(16.0)$ & $4(1.5)$ & \\
& Unemployed & $26(17.3)$ & $12(4.5)$ & \\
\hline Level of & Did not attend school & $32(21.3)$ & $6(2.2)$ & $<\mathbf{0 . 0 0 0 1}$ \\
& Primary school education only & $42(28.0)$ & $12(4.5)$ & \\
& Secondary school education & $41(27.3)$ & $44(16.5)$ & \\
& Tertiary education & $35(23.3)$ & $205(76.8)$ & \\
\hline Life events & Yes & $45(30.0)$ & $45(16.9)$ & $\mathbf{0 . 0 0 2}$ \\
& No & $105(70.0)$ & $222(83.1)$ & \\
\hline Chronic illness & Yes & $70(53.3)$ & $42(15.4)$ & $<\mathbf{0 . 0 0 0 1}$ \\
& No & $70(47.7)$ & $225(84.6)$ & \\
\hline
\end{tabular}

There were statistically significant differences in all the demographic or independent variables between the two samples. Table 5.2 shows that males were dominant in both samples, but they were more dominant in the dialysis sample (approximately $71 \%$ compared with $57 \%$ in the community sample). There were differences in the distribution of the ethnicities between the two samples: UAE Nationals accounted for approximately a quarter of the dialysis sample compared with one eighth in the community sample. More than half the community sample respondents were Arab Nationals compared with just over a third in the dialysis sample. The proportion of married respondents in both samples was almost the same. However, the percentage of widows and divorced respondents was higher in the dialysis sample (15\%) compared with the community sample (5\%). 
The majority of both samples were Muslims. Christians constituted more than one quarter of the community sample and only a tenth of the dialysis sample. The vast majority of the community sample had full-time jobs ( $80 \%$ compared with $37 \%$ in the dialysis sample). Around one fifth of the dialysis sample did not attend any schooling, and the remaining had attended primary, secondary or tertiary education. Of the 47 who had attended secondary school, only 28 reported they had received a school certificate. In contrast, approximately three quarters of the community sample had tertiary education, with a very low percentage $(2.2 \%)$ not attending any schooling, and the remainder having attended primary or secondary school. Of the 44 who had attended secondary school, only 33 reported they had received a school certificate. Of the 205 respondents who had a tertiary education, $138(31.7 \%)$ had a degree. Also, $30 \%$ of the dialysis sample had experienced a major life event such as death or marriage in the last year compared with $17 \%$ in the community sample $(p=0.002)$. More than half of the dialysis sample suffered from chronic illnesses other than kidney failure, whereas only one eighth of respondents from the community sample had on-going chronic illness ( $p=<0.0001$ ). However, data were not collected on the types of life events or on the types of chronic illnesses. Approximately one third did not know the cause of their kidney failure. A summary of the findings from the differences in the continuous socio-demographic for both samples is presented in Table 5.3.

Table 5.3 Comparison of continuous socio-demographic factors for both samples

\begin{tabular}{|l|r|r|r|}
\hline Variable & $\begin{array}{c}\text { Dialysis Mean } \\
\mathbf{\pm S D} \text { and range } \\
(\mathbf{n}=\mathbf{1 5 0})\end{array}$ & $\begin{array}{c}\text { Community } \\
\text { Mean } \pm \text { SD and } \\
\text { range }(\mathbf{n}=\mathbf{2 6 7})\end{array}$ & p value \\
\hline Age in years & $\begin{array}{c}49.1 \pm 12.2 \\
19-86 \text { years }\end{array}$ & $\begin{array}{c}40.5 \pm 11.3 \\
18-69 \text { years }\end{array}$ & $<\mathbf{0 . 0 0 0 1}$ \\
\hline Time lived in UAE & $26.8 \pm 14.2$ & $15.7 \pm 13.3$ & $<\mathbf{0 . 0 0 0 1}$ \\
& $0-86$ years & $0.16-64$ years & \\
\hline Time travelled outside of UAE & $3.9 \pm 6.0$ & $1.45 \pm 3.0$ & $<\mathbf{0 . 0 0 0 1}$ \\
& $0-40$ years & $0-37$ years & \\
\hline
\end{tabular}

t-test

The dialysis sample covered a wide range of ages from 19 to 86 years. Over half $(53.4 \%)$ of the sample were over 50 years. In contrast, in the community sample the youngest was 18 and the oldest 69 years. Although not apparent in the table, three quarters of the community sample were younger than 50 years. The dialysis sample was older in average by 8.6 years compared with the community sample. Moreover, the dialysis sample had 
lived longer in the UAE compared with the community sample. The time lived in or travelled out of UAE was presented in years in the above table in order to have one denominator and to facilitate comparison. The average last time the dialysis respondents had travelled outside of the UAE was double that of the community sample. The average time lived in UAE was 26.8 years.

\subsection{Missing data}

All questions on the demographic variables for the dialysis and the community samples were completed by all respondents. However, not all respondents completed all questions in each of the QOL tools. The questionnaire was excluded from data analysis if responses in one or more subscales were missing. The data showed that one respondent from the dialysis sample did not complete one question from the vitality subscale in the SF-36; this question was replaced with the average of the remaining vitality subscale questions. There were eight respondents from the community sample who missed answering questions in the SF-36. Two respondents missed one question from the social and functioning subscales, one missed three questions from the general health subscale and one missed one question from the vitality and one from the mental health subscales. The missing data were replaced with the mean of that respective subscale as per the instructions of the authors of the SF-36. Although there were 23 different questions missed by the community sample in the SF-36, given that few questions were missed by any given individual, the analyses were able to be calculated using the full sample. Most questions were only missed by one or two respondents. The sample size for the community was reduced by three respondents as these individuals did not complete more than half of the questions related to different subscales.

The demographic areas known to have a direct impact on the cultural relevancy of the tools are presented in Table 5.4 and 5.5. Table 5.4 provides details of the summary characteristics of respondents who missed questions on the SF-36 and Table 5.5 provides details of the summary characteristics of respondents who missed questions on the QOL Index. 
Table 5.4 Characteristics of respondents who missed questions on the SF-36

\begin{tabular}{|l|l|r|r|r|r|r|}
\hline \multirow{2}{*}{} & \multicolumn{6}{|c|}{ Number (\%) of questions missed } \\
\cline { 3 - 7 } & Number & None & $\mathbf{1 - 4}$ & $\mathbf{5 - 9}$ & $\mathbf{1 0 +}$ \\
\hline Group & Dialysis & 150 & $145(97)$ & $5(3)$ & $0(0)$ & $0(0)$ \\
& Community & 267 & $254(95)$ & $12(5)$ & $1(0.5)$ & $0(0)$ \\
\hline Gender & Male & 259 & $245(94)$ & $14(5)$ & $0(0)$ & $1(1)$ \\
& Female & 159 & $156(98)$ & $3(2)$ & $0(0)$ & $0(0)$ \\
\hline Ethnicity & UAE National & 74 & $72(97)$ & $2(3)$ & $0(0)$ & $0(0)$ \\
& Arab National & 193 & $186(96)$ & $6(3)$ & $0(0)$ & $1(1)$ \\
& South East Asian & 97 & $92(95)$ & $5(5)$ & $0(0)$ & $0(0)$ \\
& Other & 53 & $49(92)$ & $4(8)$ & $0(0)$ & $0(0)$ \\
\hline Religion & Muslim & 303 & $291(96)$ & $11(4)$ & $0(0)$ & $1(0)$ \\
& Christian & 88 & $87(99)$ & $1(1)$ & $0(0)$ & $0(0)$ \\
& Other & 26 & $21(81)$ & $5(19)$ & $0(0)$ & $0(0)$ \\
\hline
\end{tabular}

*Percentages rounded up to whole numbers

From this table, it can be seen that the majority of both samples missed no questions. Very few missed 1-4 questions. Five per cent of respondents from the community sample missed 1-4 questions compared to $3 \%$ in the dialysis sample. Demographic findings indicated that males proportionally missed more questions than females. Respondents who belonged to other ethnicities missed also more questions. Respondents who belonged to other religions missed more questions than Muslims and Christians.

There were more questions that were not answered by participants in the QOL Index by both samples compared with the SF-36.

Table 5.5 Characteristics of respondents who missed questions on the QOL index

\begin{tabular}{|l|l|r|r|r|r|r|}
\hline \multicolumn{2}{|c|}{} & & \multicolumn{3}{|c|}{ No (\%) of questions missed } \\
\cline { 3 - 7 } \multicolumn{2}{c|}{} & Number & None & $\mathbf{1 - 4}$ & $\mathbf{5 - 9}$ & \multicolumn{1}{|c|}{$\mathbf{1 0 +}$} \\
\hline Group & Dialysis & 150 & $3(2)$ & $113(75)$ & $31(21)$ & $3(2)$ \\
& Community & 267 & $3(1)$ & $203(76)$ & $44(16)$ & $17(6)$ \\
\hline Gender & Male & 259 & $3(1)$ & $208(80)$ & $40(13)$ & $8(4)$ \\
& Female & 159 & $3(1)$ & $110(70)$ & $35(22)$ & $11(7)$ \\
\hline Ethnicity & UAE National & 74 & $1(1)$ & $52(74)$ & $15(20)$ & $6(8)$ \\
& Arab National & 193 & $3(2)$ & $145(75)$ & $33(17)$ & $12(6)$ \\
& South East Asian & 97 & $2(2)$ & $76(78)$ & $17(18)$ & $2(2)$ \\
& Other & 53 & $1(2)$ & $42(79)$ & $10(2)$ & $0(0)$ \\
\hline Religion & Muslim & 303 & $1(0)$ & $224(74)$ & $60(20)$ & $18(6)$ \\
& Christian & 88 & $1(1)$ & $73(83)$ & $12(14)$ & $2(2)$ \\
& Other & 26 & $1(4)$ & $22(85)$ & $3(12)$ & $0(0)$ \\
\hline
\end{tabular}

${ }^{*}$ Percentages rounded up to whole numbers 
From the above table, it can be seen that more questions were missed by respondents from both samples when using the QOL Index. Few respondents from both samples missed no questions. The majority of the respondents from the community missed 1-4 questions proportionately. More male respondents missed 1-4 questions than female. The percentage of the different ethnic and religion groups for the respondents who missed 1-4 questions were similar. The percentage of female respondents who missed 5-9 questions was higher than the male respondents. UAE Nationals missed 5-9 questions more frequently than other ethnic groups. Questions that were missed by more than $10 \%$ of the respondents were examined for elements of cultural relevancy. Details of these are summarised in Table 5.6.

Table 5.6 Questions that had highest missing responses in the QOL Index tool

\begin{tabular}{|l|l|l|r|r|r|}
\hline $\begin{array}{l}\text { QOL Index } \\
\text { Question \# }\end{array}$ & Subscale & Content & $\begin{array}{c}\text { Dialysis } \\
\mathbf{n}(\%)\end{array}$ & $\begin{array}{c}\text { Community } \\
\mathbf{n}(\%)\end{array}$ & Total \\
\hline qolis8 & $\begin{array}{l}\text { Health and } \\
\text { Functioning }\end{array}$ & $\begin{array}{l}\text { Satisfaction with your chances } \\
\text { of living as long as you would } \\
\text { like? }\end{array}$ & $9(6)$ & $30(11)$ & $39(17)$ \\
\hline qolis12 & $\begin{array}{l}\text { Health and } \\
\text { Functioning }\end{array}$ & Satisfaction with sex life & $24(16)$ & $37(14)$ & $61(30)$ \\
\hline qolis13 & Family & $\begin{array}{l}\text { Satisfaction with spouse, } \\
\text { lover, or partner? }\end{array}$ & $25(17)$ & $37(14)$ & $62(31)$ \\
\hline qolii8 & $\begin{array}{l}\text { Health and } \\
\text { Functioning }\end{array}$ & $\begin{array}{l}\text { Importance of living as long as } \\
\text { you would like? }\end{array}$ & $8(5)$ & $28(11)$ & $36(16)$ \\
\hline qolii12 & $\begin{array}{l}\text { Health and } \\
\text { Functioning }\end{array}$ & Importance of sex life & $21(14)$ & $29(11)$ & $50(25)$ \\
\hline qolii13 & Family & $\begin{array}{l}\text { Importance of spouse, lover, } \\
\text { or partner? }\end{array}$ & $17(11)$ & $25(9)$ & $42(21)$ \\
\hline
\end{tabular}

*Percentages rounded up to whole numbers

The most commonly missed questions were from the health and functioning and family subscales. These questions were related to the importance and satisfaction with sex life, spouse, and chances of living as long as they would like to. The remainder of the missing responses in the QOL Index tool are presented in Table 5.7.

\subsubsection{Cultural relevancy in relation to missing data from QOL Index tool subscales}

Table 5.7 summarises the missing data in relation to subscales of QOL Index for dialysis sample. Bolding of the values that have more than $5 \%$ of missing data has been done to draw attention to the possible link between missing data and cultural relevancy. 
Table 5.7 Missing data in relation to subscales of QOL Index for dialysis sample

\begin{tabular}{|c|c|c|c|c|}
\hline Subscale & $\begin{array}{l}\text { Ques } \\
\text { tion } \\
\#\end{array}$ & Question & $\begin{array}{l}\text { Missing data } \\
\text { of satisfaction } \\
\mathrm{n}(\%)\end{array}$ & $\begin{array}{l}\text { Missing data } \\
\text { of importance } \\
n(\%)\end{array}$ \\
\hline \multirow{14}{*}{$\begin{array}{l}\text { Health and } \\
\text { Functioning } \\
\text { Subscale }\end{array}$} & 1. & Health & - & - \\
\hline & 2. & Health care & $1(0.7)$ & - \\
\hline & 3. & Energy (fatigue) & - & - \\
\hline & 4. & $\begin{array}{l}\text { Ability to take care of yourself } \\
\text { without help }\end{array}$ & $3(2.0)$ & $1(0.7)$ \\
\hline & 5. & Likelihood of kidney transplant & $5(3.3)$ & $3(2.0)$ \\
\hline & 6. & $\begin{array}{l}\text { Changes made in life because of } \\
\text { kidney failure }\end{array}$ & $4(2.7)$ & 11(7.3) \\
\hline & 7. & Control over life & - & $3(2.0)$ \\
\hline & 8. & $\begin{array}{l}\text { Chances for living as long as you } \\
\text { would like }\end{array}$ & $9(6.0)$ & $8(5.3)$ \\
\hline & 12. & Sex life & 24(16.0) & 21(14.0) \\
\hline & 17. & $\begin{array}{l}\text { Ability to take care of family } \\
\text { responsibilities }\end{array}$ & $2(1.3)$ & $3(2.0)$ \\
\hline & 18. & Usefulness to others & $4(2.7)$ & $2(1.3)$ \\
\hline & 19. & Worries & - & $1(0.7)$ \\
\hline & 26. & Things for fun & $2(1.3)$ & - \\
\hline & 27. & Chances for a happy future & $1(0.7)$ & $1(0.7)$ \\
\hline \multirow{7}{*}{$\begin{array}{l}\text { Social and } \\
\text { Economic } \\
\text { Subscale }\end{array}$} & 14. & Friends & $0(0.0)$ & - \\
\hline & 16. & $\begin{array}{l}\text { Emotional support from people } \\
\text { other than your family }\end{array}$ & $4(2.7)$ & $4(2.7)$ \\
\hline & 20. & Neighborhood & $4(2.7)$ & $1(0.7)$ \\
\hline & 21. & Home & $6(4.0)$ & $3(2.0)$ \\
\hline & $22 / 23$ & Job/not having a job & $\begin{array}{l}58(38.7) \\
86(57.3)\end{array}$ & $\begin{array}{l}53(35.3) \\
94(62.7)\end{array}$ \\
\hline & 24. & Education & $5(3.3)$ & $4(2.7)$ \\
\hline & 25. & Financial needs & $4(2.7)$ & $3(2.0)$ \\
\hline \multirow{7}{*}{$\begin{array}{l}\text { Psychologic } \\
\text { al/Spiritual } \\
\text { Subscale }\end{array}$} & 28. & Peace of mind & $3(2.0)$ & - \\
\hline & 29. & Faith in God & $1(0.7)$ & - \\
\hline & 30. & Achievement of personal goals & $2(1.3)$ & - \\
\hline & 31. & Happiness in general & $1(0.7)$ & $1(0.7)$ \\
\hline & 32. & Life satisfaction in general & $3(2.0)$ & - \\
\hline & 33. & Personal appearance & $2(1.3)$ & $1(0.7)$ \\
\hline & 34. & Self & $2(1.3)$ & $1(0.7)$ \\
\hline \multirow{5}{*}{$\begin{array}{l}\text { Family } \\
\text { Subscale }\end{array}$} & 9. & Family health & $1(0.7)$ & $1(0.7)$ \\
\hline & 10. & Children & 11(7.3) & $7(4.7)$ \\
\hline & 11. & Family happiness & $5(3.3)$ & $5(3.3)$ \\
\hline & 13. & Spouse, lover, or partner & 24(16.0) & 17(11.3) \\
\hline & 15. & Emotional support from family & $1(0.7)$ & $1(0.7)$ \\
\hline
\end{tabular}

The above table shows that satisfaction and importance of chances for living as long as they would like and sex life items in the health and functioning subscale for the dialysis 
sample had the highest proportions of missing data. Table 5.8 presented the missing data in relation to subscales of QOL Index for community sample.

Table 5.8 Missing data in relation to subscales of QOL Index for community sample

\begin{tabular}{|c|c|c|c|c|}
\hline Subscale & $\begin{array}{l}\text { Quest } \\
\text { ion \# }\end{array}$ & Question & $\begin{array}{l}\text { Missing data } \\
\text { of satisfaction } \\
\mathrm{n}(\%)\end{array}$ & $\begin{array}{l}\text { Missing data } \\
\text { of importance } \\
n(\%)\end{array}$ \\
\hline \multirow{13}{*}{$\begin{array}{l}\text { Health and } \\
\text { Functioning } \\
\text { Subscale }\end{array}$} & 1. & Health & $1(0.4)$ & - \\
\hline & 2. & Health care & $4(1.5)$ & $4(1.5)$ \\
\hline & 3. & Pain & $28(10.5)$ & $5(1.9)$ \\
\hline & 4. & Energy (fatigue) & $9(3.4)$ & $2(0.7)$ \\
\hline & 5. & $\begin{array}{l}\text { Ability to take care of yourself } \\
\text { without help }\end{array}$ & $6(2.2)$ & $4(1.5)$ \\
\hline & 6. & Control over life & 12(4.5) & $7(2.6)$ \\
\hline & 7. & $\begin{array}{l}\text { Chances for living as long as } \\
\text { you would like }\end{array}$ & $30(11.2)$ & $28(10.5)$ \\
\hline & 11. & Sex life & $37(13.9)$ & $29(10.9)$ \\
\hline & 16. & $\begin{array}{l}\text { Ability to take care of family } \\
\text { responsibilities }\end{array}$ & $4(1.5)$ & $1(0.4)$ \\
\hline & 17. & Usefulness to others & $10(3.7)$ & $6(2.2)$ \\
\hline & 18. & Worries & $13(4.9)$ & $3(1.1)$ \\
\hline & 25. & Things for fun & $4(1.5)$ & - \\
\hline & 26. & Chances for a happy future & $3(1.1)$ & $2(0.7)$ \\
\hline \multirow{7}{*}{$\begin{array}{l}\text { Social and } \\
\text { Economic } \\
\text { Subscale }\end{array}$} & 13. & Friends & $6(2.2)$ & $6(2.2)$ \\
\hline & 15. & $\begin{array}{l}\text { Emotional support from people } \\
\text { other than your family }\end{array}$ & $6(2.2)$ & $6(2.2)$ \\
\hline & 19. & Neighborhood & $6(2.2)$ & $3(1.1)$ \\
\hline & 20. & Home & $5(1.9)$ & $7(2.6)$ \\
\hline & $21 / 22$ & Job/not having a job & $\begin{array}{r}38(14.2) / \\
232(85.8)\end{array}$ & $\begin{array}{r}36(13.5) / \\
225(86.5)\end{array}$ \\
\hline & 23. & Education & $2(0.7)$ & $7(2.6)$ \\
\hline & 24. & Financial needs & $4(1.5)$ & $1(0.4)$ \\
\hline \multirow{7}{*}{$\begin{array}{l}\text { Psychological/ } \\
\text { Spiritual } \\
\text { Subscale }\end{array}$} & 27. & Peace of mind & $3(1.1)$ & - \\
\hline & 28. & Faith in God & - & $1(0.4)$ \\
\hline & 29. & Achievement of personal goals & $3(1.1)$ & $2(0.7)$ \\
\hline & 30. & Happiness in general & $3(1.1)$ & $3(1.1)$ \\
\hline & 31. & Life satisfaction in general & - & - \\
\hline & 32. & Personal appearance & - & - \\
\hline & 33. & Self & - & $2(0.7)$ \\
\hline \multirow{5}{*}{$\begin{array}{l}\text { Family } \\
\text { Subscale }\end{array}$} & 8. & Family health & $6(2.2)$ & $7(2.6)$ \\
\hline & 9. & Children & 44(16.5) & $31(11.6)$ \\
\hline & 10. & Family happiness & 14(5.2) & 17(6.4) \\
\hline & 12. & Spouse, lover, or partner & $37(13.9)$ & 26(9.4) \\
\hline & 14. & Emotional support from family & $5(1.9)$ & - \\
\hline
\end{tabular}

This table showed that the satisfaction and importance of chances for living as long as they would like and the sex life items in the health and functioning subscale for the 
community sample had the highest proportions of missing data $(11.2 \%, 10.5 \%$ and $13.9 \%$, $10.9 \%$ respectively).

Further details on the missing data from the SF-36 for the community sample are presented in Appendix 9. In general, the percentages of missing data from both samples were statistically insignificant.

\subsection{Data distribution}

This section reports on data distribution for the combined samples using mean and median, kolmogorov-smirnov Z test, skewness and kurtosis, normality plots, and Q-Q plot tests.

To establish the data distributions in this study both samples were combined as one with a total number of respondents of 414 . The mean of the total scores of the SF-36 for the combined sample was 69.19 and the median 72.59, while the mean for the QOL Index was 23.43 and the median was 24.11. The differences between the mean and the median for the SF-36 and the QOL Index are minimal, suggesting a normal distribution of the data.

The Kolmogorov-Smirnov Z (KS) test results were $<0.0001$ for both the total scores of the SF-36 and QOL Index tools. This indicates that the data were not normally distributed because the $p$ values for the two scales were less than 0.05 . However, the major limitation of the KS test of normality is that it is very sensitive to extreme values that cause the tail of distribution.

The values of both tools were negatively skewed: -0.561 for total scores of the SF-36 and 0.800 for the total scores QOL Index. The scores were clustered at the high end (left side of the graph). Any values above, between +3 and -3 are considered as an indication that the variable is not normally distributed as per Peat and Barton (2006). Kurtosis values of both tools differed the SF-36 was negative -0.578 and the QOL Index positive +0.125 . As per Peat and Barton, a kurtosis value above 1.00 indicates that the distribution tends to be pointed and a value below 1 indicates that the distribution tends to be flat. In this study, skewness and kurtosis values between -1.00 and +1.00 indicate normality. The actual 
shapes of distribution of the total scores of the SF-36 and QOL Index tools were clearly seen to approximate the bell curve shape in the histograms. Also, the outlying values were on the left side of the graph. Normality plots for the tools are demonstrated in Figures 5.1 and 5.2 .

Figure 5.1 Normality plot of the combined SF-36 total scores

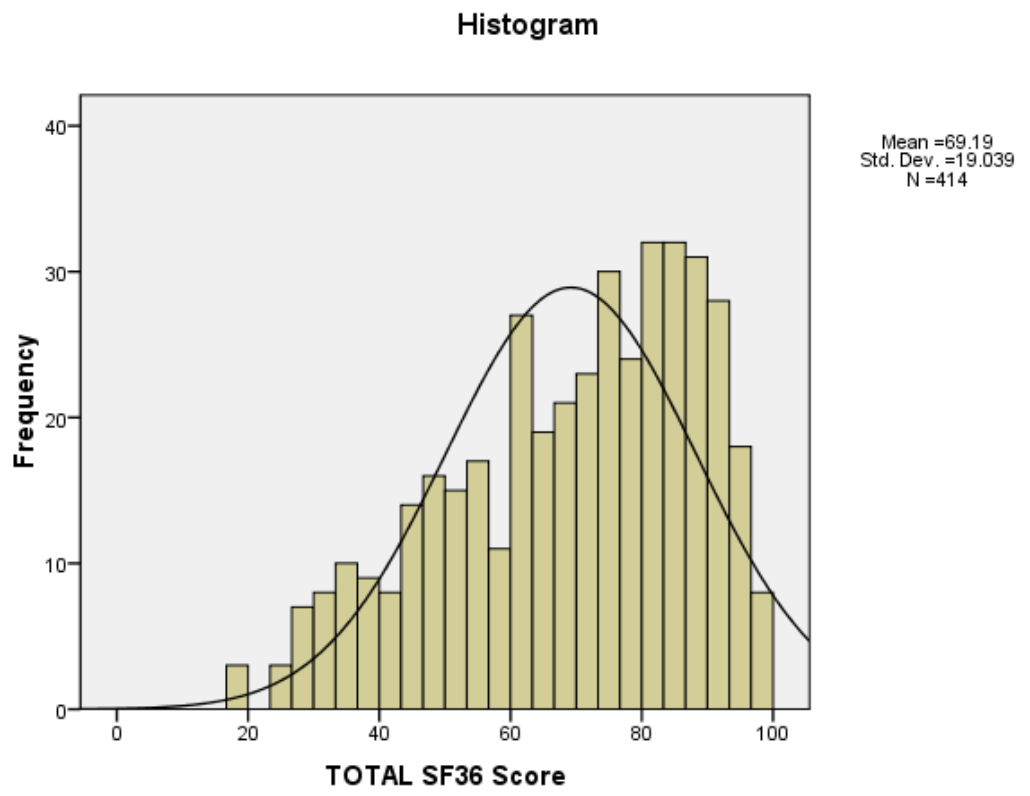


Figure 5.2 Normality plot of the combined QOL Index total scores

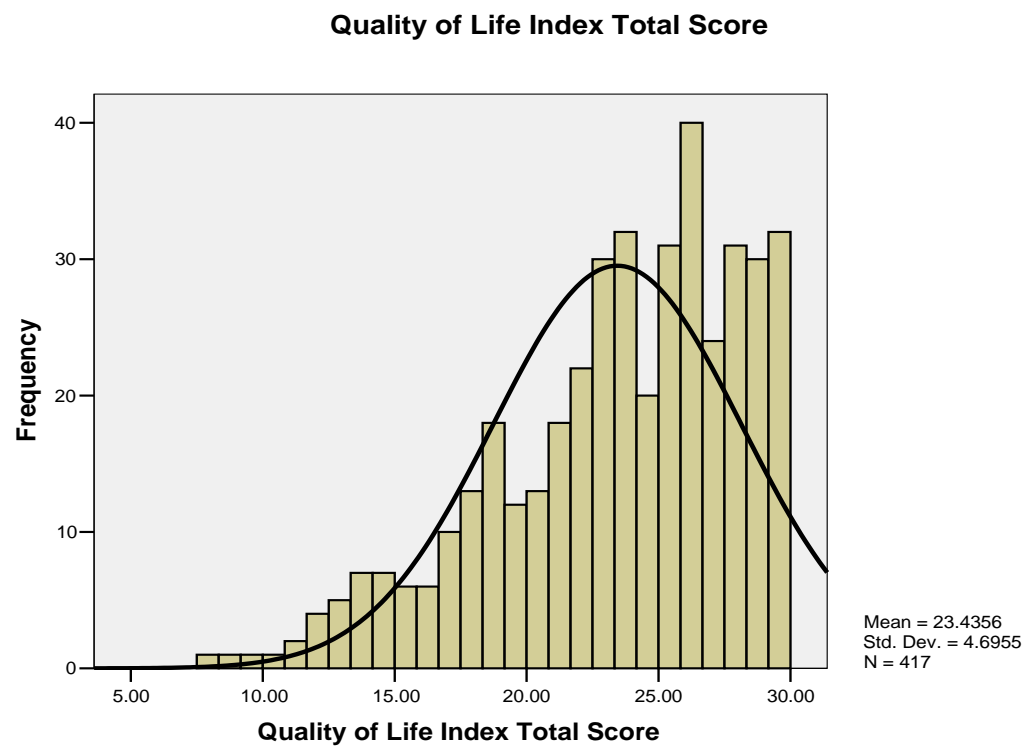

The Q-Q plots for total scores of SF-36 and the QOL index showed that the points are clustered randomly around the horizontal line at zero with a few points spread below and above the line as demonstrated in Figures 5.3 and 5.4. If the distribution is not normal, the points will be in a pattern such as a $\mathrm{J}$ or an inverted $\mathrm{U}$ distribution and the horizontal line may not be at the centre of the data (Peat \& Barton, 2006). The normal Q-Q plot for the total scores of SF-36 and the QOL Index are shown in Figures 5.3 and 5.4. 
Figure 5.3 Normal Q-Q plot for the combined SF-36 total scores

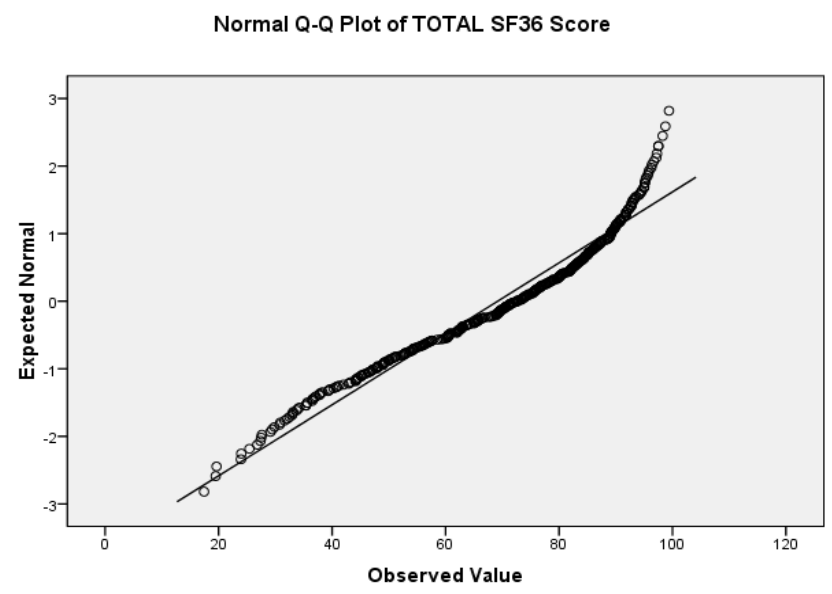

Figure 5.4 Normal Q-Q plot for the combined QOL Index total scores

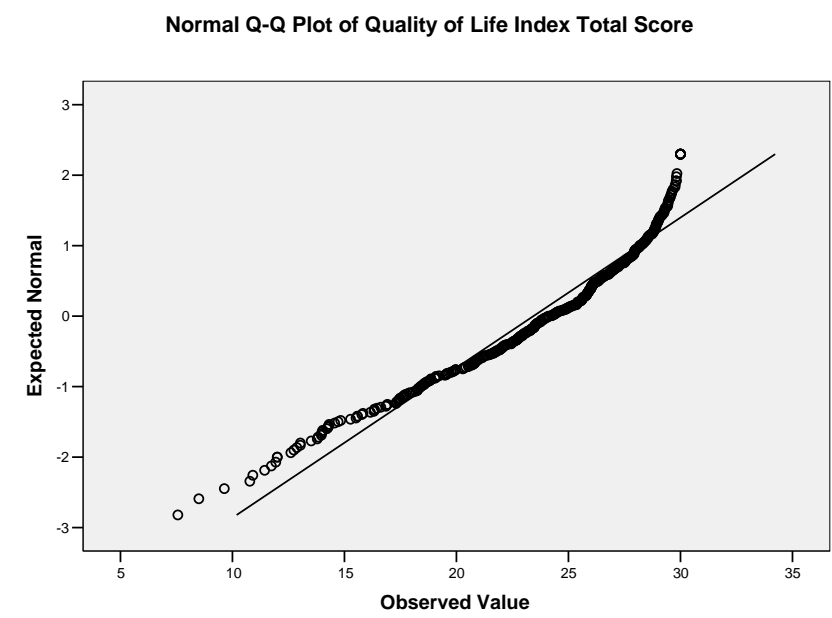

Table 5.9 summarises the statistical tests used to investigate data distribution. In the Table, "yes" indicates that the distribution is within the normal range and "no" indicates that the distribution is outside the normal range. 
Table 5.9 Summary of data distribution tests

\begin{tabular}{|l|r|l|r|r|r|r|r|}
\hline & $\begin{array}{l}\text { Mean- } \\
\text { median }\end{array}$ & $\begin{array}{l}\text { (KS) } \\
\text { test }\end{array}$ & $\begin{array}{l}\text { Skewness } \\
\text { \& Kurtosis }\end{array}$ & $\begin{array}{l}\text { Normality } \\
\text { plots }\end{array}$ & $\begin{array}{l}\text { Q-Q } \\
\text { Plots }\end{array}$ & $\begin{array}{l}\text { Extreme } \\
\text { values \& } \\
\text { outliers test }\end{array}$ & $\begin{array}{l}\text { Overall } \\
\text { decision }\end{array}$ \\
\hline SF-36 & Yes & No & Yes & Yes & Yes & Yes & Yes \\
\hline QOL Index & Yes & No & Yes & Yes & Yes & Yes & Yes \\
\hline
\end{tabular}

Overall the descriptive statistics for data distribution indicated a normal distribution of the data. This justifies the use of parametric tests to establish a comparative analysis between the QOL tools, the SF-36 and the QOL Index.

\subsection{Cultural relevancy of tools}

This section of the findings presents the cultural relevancy of the SF-36 and the QOL Index for both samples. This finding presents a comparison of the cultural relevancy of the SF-36 and the QOL Index between the dialysis and the community samples. A comparison of the cultural relevancies of both tools is outlined in Table 5.10 .

Table 5.10 Comparison between the cultural relevancies of both tools

\begin{tabular}{|l|l|r|r|}
\hline Variable & Coding & Dialysis (150) $\mathbf{n ( \% )}$ & Community (267) $\mathbf{n ( \% )}$ \\
\hline SF-36 considered relevant & Yes & $142(94.7)$ & $242(90.6)$ \\
to culture & No & $8(5.3)$ & $25(9.4)$ \\
\hline QOL Index considered & Yes & $143(95.3)$ & $254(95.1)$ \\
relevant to culture & No & $7(4.7)$ & $13(4.9)$ \\
\hline Which tool is more & Neither tool & $4(2.7)$ & $6(2.2)$ \\
culturally relevant? & SF-36 & $9(6.0)$ & $24(9.0)$ \\
& QOLI & $47(31.3)$ & $45(16.9)$ \\
& Either tool & $90(60.0)$ & $192(71.9)$ \\
\hline
\end{tabular}

The result from the above table showed that both tools were considered culturally relevant by the vast majority of respondents from both samples. Although both tools were culturally relevant, $31.3 \%$ of the respondents from the dialysis sample considered that the QOL Index was more culturally relevant. The above table indicates that $90(60 \%)$ of the dialysis sample considered that either tools were culturally relevant compared with $192(72 \%)$ in the community sample. The table also indicates that few respondents in each sample considered that neither tool was culturally relevant. Table 5.9 showed that $5.3 \%$ of the dialysis sample and $9.4 \%$ from the community sample considered the SF-36 not culturally relevant. Moreover, $4.7 \%$ of the dialysis sample and $4.9 \%$ from the community sample 
considered the QOL Index not culturally relevant. The demographic characteristics of these respondents from both samples are summarised in Table 5.11.

Table 5.11 Demographic characteristics of the respondents who considered QOL tools were not culturally relevant by sample

\begin{tabular}{|c|c|c|c|c|c|}
\hline \multirow[t]{2}{*}{ Variable } & \multirow[t]{2}{*}{ Values } & \multicolumn{2}{|r|}{ SF-36 } & \multicolumn{2}{|c|}{ QOL Index } \\
\hline & & $\begin{array}{c}\text { Dialysis (8) } \\
\mathrm{n}(\%)\end{array}$ & $\begin{array}{l}\text { Community (25) } \\
\mathrm{n}(\%)\end{array}$ & $\begin{array}{l}\text { Dialysis (7) } \\
\mathrm{n}(\%)\end{array}$ & $\begin{array}{l}\text { Community (13) } \\
\mathrm{n}(\%)\end{array}$ \\
\hline Gender & $\begin{array}{l}\text { Female } \\
\text { Male }\end{array}$ & $\begin{array}{l}3(38) \\
5(62)\end{array}$ & $\begin{array}{r}9(36) \\
16(64)\end{array}$ & $\begin{array}{l}3(43) \\
4(57)\end{array}$ & $\begin{array}{r}3(23) \\
10(77)\end{array}$ \\
\hline Ethnicity & $\begin{array}{l}\text { UAE National } \\
\text { Arab National } \\
\text { South Asian } \\
\text { Other }\end{array}$ & $\begin{array}{r}0(0) \\
2(24) \\
3(38) \\
3(38) \\
\end{array}$ & $\begin{array}{r}2(8) \\
6(24) \\
9(36) \\
8(32) \\
\end{array}$ & $\begin{array}{c}1(14) \\
1(14) \\
3(43) \\
2(28) \\
\end{array}$ & $\begin{array}{r}1(8) \\
1(8) \\
6(46) \\
5(38) \\
\end{array}$ \\
\hline $\begin{array}{l}\text { Marital } \\
\text { status }\end{array}$ & $\begin{array}{l}\text { Single } \\
\text { Married } \\
\text { Divorced or } \\
\text { widowed }\end{array}$ & $\begin{array}{r}2(24) \\
6(76) \\
0(0)\end{array}$ & $\begin{array}{r}3(12) \\
21(84) \\
1(4)\end{array}$ & $\begin{array}{l}1(14) \\
3(43) \\
3(43)\end{array}$ & $\begin{array}{r}3(23) \\
10(77) \\
0(0)\end{array}$ \\
\hline Religion & $\begin{array}{l}\text { Muslim } \\
\text { Christian } \\
\text { Others }\end{array}$ & $\begin{array}{l}3(38) \\
3(38) \\
2(24)\end{array}$ & $\begin{array}{r}1(4) \\
11(44) \\
13(52)\end{array}$ & $\begin{array}{l}3(43) \\
3(43) \\
1(14)\end{array}$ & $\begin{array}{l}3(24) \\
8(60) \\
2(16)\end{array}$ \\
\hline Education & $\begin{array}{l}\text { Did not attend } \\
\text { school } \\
\text { Primary school } \\
\text { Secondary } \\
\text { school } \\
\text { Tertiary } \\
\text { education }\end{array}$ & $\begin{array}{r}0 \\
4(50) \\
2(25) \\
2(25)\end{array}$ & $\begin{array}{r}0(0) \\
2(8) \\
11(44) \\
12(48)\end{array}$ & $\begin{array}{r}0(0) \\
2(28) \\
3(44) \\
1(14)\end{array}$ & $\begin{array}{r}0(0) \\
0(0) \\
10(77) \\
3(23)\end{array}$ \\
\hline
\end{tabular}

Although the denominator is small for both samples, percentages were calculated for comparative purposes. From the above table, it can be seen that the percentage of males who considered both tools were not culturally relevant is greater than the number of females. Furthermore, the majority of respondents in both samples, who considered that both tools were not culturally relevant belonged to a South East Asian ethnicity and were married. The majority of respondents in the dialysis sample, who considered that the SF36 was not culturally relevant, belonged to either Muslim or Christian religions and had attended primary schools. While in the community sample the majority of respondents, who considered that the SF-36 was not culturally relevant belonged to others religion, had tertiary education and attended secondary schools. The majority of respondents in the dialysis sample, who considered that the QOL Index was not culturally relevant belonged 
to Muslim and Christian religions and had attended secondary schools. While in the community sample they belonged to Christian religion and had attended secondary schools.

As part of the survey, respondents were asked what, if any, questions should be added or deleted to the tools to make them more culturally relevant. Twenty five $(16.6 \%)$ of the dialysis respondents suggested adding or deleting questions from both tools. Of these $17(11.3 \%$ ) respondents suggested adding questions to the SF-36, but suggested deleting none. On the other hand, 11(7.3\%) dialysis respondents suggested adding questions to and $4(2.6 \%)$ respondents suggested deleting questions from the QOL Index. The nature of the suggested added and deleted questions will be discussed in Chapter 7. Three respondents did not suggest adding or deleting questions but commented on either tool. The first respondent commented "the tools are excellent \& applicable to renal people" and the second respondent commented "some of the questions that have a well-known answers do not require a reaction or an answer in the QOL Index tool". The third respondent commented "all the questions match the beliefs and values of people living in the UAE'”.

In the community sample, 49(18.4\%) respondents suggested deleting or adding questions to the tools. Of these 49 respondents, 38(14.2\%) respondents suggested adding questions and three respondents suggested deleting questions from the SF-36. In contrast, 10 $(3.7 \%)$ respondents suggested adding questions and 10 suggested deleting questions from the QOL Index tool. There were 4(1.5\%) respondents who did not suggest adding or deleting questions but commented on either tool. The first respondent commented "As a solution to decrease life expenses, government should stop the agents from increasing the house rent". The second respondent commented "The SF-36 refers to experiences of the last 4 weeks, but the person may have suffered from the disease for longer than this period which is not captured in this survey, In general it is a good survey". The third respondent commented "The questions do not match the culture and the environment we live in. It may match people live in Europe". The fourth commented "Even in anonymous surveys people do not disclose their disease to keep their jobs". 
Respondents from the dialysis sample were also asked how good are the SF-36 and QOL Index tools in capturing the quality of their life. A summary of their responses is presented in Table 5.12

Table 5.12 Dialysis respondents' opinion on the QOL tools

\begin{tabular}{|l|r|r|}
\hline \multicolumn{1}{|c|}{ Responses } & SF-36 n(\%) & QOL Index tool $\mathbf{n}(\%)$ \\
\hline Excellent & $30(20.0)$ & $36(24.0)$ \\
\hline Very good & $18(12.0)$ & $34(22.7)$ \\
\hline Good & $99(66.0)$ & $75(50.0)$ \\
\hline Poor & $2(1.3)$ & $5(3.3)$ \\
\hline
\end{tabular}

Two thirds of the dialysis respondents rated the SF-36 as good in capturing their QOL compared with half for the QOL Index. One fifth of the dialysis respondents rated the SF36 as excellent in capturing their QOL compared with almost one quarter for the QOL Index. Very few considered that both tools were poor in capturing their QOL.

\subsection{Findings from qualitative data}

This section reports on the missing data from the qualitative part and presents the findings from the three open-ended questions. The missing data of the open-ended question are outlined in Table 5.13.

Table 5.13 Missing data of the open-ended questions

\begin{tabular}{|l|r|r|}
\hline Questions & \multicolumn{1}{|c|}{$\begin{array}{c}\text { No of times } \\
\text { respondents missed } \\
\text { questions in the } \\
\text { Dialysis sample } \mathbf{n}(\%)\end{array}$} & $\begin{array}{c}\text { No of times } \\
\text { respondents missed } \\
\text { questions in the } \\
\text { Community sample } \mathbf{n} \\
(\%)\end{array}$ \\
\hline $\begin{array}{l}3 \text { things people living in UAE value most in } \\
\text { life }\end{array}$ & $27(18.0)$ & $36(13.5)$ \\
\hline 3 things you personally value most in life & $15(10.0)$ & $17(6.4)$ \\
\hline $\begin{array}{l}3 \text { things most important things to you in } \\
\text { maintaining or improving your quality of life }\end{array}$ & $28(18.6)$ & $47(17.6)$ \\
\hline
\end{tabular}

The number of respondents who missed answering the first and the third open-ended questions was almost double the number of respondents who missed answering the second question. In general the percentage of missing data for the dialysis sample is $15.5 \%$ compared to $12.5 \%$ in the community sample. Question three had the highest percentage of missing data in both samples (18.6\% and $17.6 \%$ respectively). Furthermore, 
not all respondents named three things each time. Analysis of the responses to the three open-ended questions resulted in certain themes emerging (Table 5.14). In this table the themes were listed in the order of total responses by the combined samples. Further information on ranking of these themes is presented later in this chapter.

Table 5.14 Responses of open-ended questions for both samples

\begin{tabular}{|l|r|r|r|r|r|r|}
\hline & \multicolumn{2}{|c|}{ Question 1 } & \multicolumn{2}{c|}{ Question 2 } & \multicolumn{2}{c|}{ Question 3 } \\
\hline & $\begin{array}{c}\text { Dialysis } \\
\mathbf{n}(\%)\end{array}$ & $\begin{array}{c}\text { Community } \\
\mathbf{n}(\%)\end{array}$ & $\begin{array}{c}\text { Dialysis } \\
\mathbf{n}(\%)\end{array}$ & $\begin{array}{c}\text { Community } \\
\mathbf{n}(\%)\end{array}$ & $\begin{array}{c}\text { Dialysis } \\
\mathbf{n}(\%)\end{array}$ & $\begin{array}{c}\text { Community } \\
\mathbf{n}(\%)\end{array}$ \\
\hline Health & $58(38.6)$ & $124(44.9)$ & $91(60.7)$ & $183(66.1)$ & $55(36.7)$ & $77(27.9)$ \\
\hline Money & $61(40.7)$ & $126(45.7)$ & $37(24.7)$ & $58(20.9)$ & $41(27.3)$ & $106(38.4)$ \\
\hline Family & $26(17.3)$ & $86(31.2)$ & $75(50.0)$ & $183(66.1)$ & $28(18.7)$ & $46(16.7)$ \\
\hline Happy life & $43(28.7)$ & $69(25.0)$ & $29(19.3)$ & $51(18.4)$ & $29(19.3)$ & $49(17.8)$ \\
\hline Religion & $27(18.0)$ & $49(17.8)$ & $46(30.7)$ & $79(28.5)$ & $21(14.0)$ & $31(11.2)$ \\
\hline Safety & $37(24.7)$ & $43(15.6)$ & $24(16.0)$ & $40(14.4)$ & $24(16.0)$ & $50(18.1)$ \\
\hline Relationship & $13(8.7)$ & $24(8.7)$ & $21(14.0)$ & $18(6.5)$ & $25(16.7)$ & $34(12.3)$ \\
\hline Job & $21(14.0)$ & $37(13.4)$ & $12(8.0)$ & $40(14.4)$ & $19(12.7)$ & $69(25.0)$ \\
\hline Housing & $16(10.7)$ & $22(8.0)$ & $7(4.7)$ & $11(4.0)$ & $11(7.3)$ & $16(5.8)$ \\
\hline Education & $6(4.0)$ & $23(8.3)$ & $10(6.7)$ & $29(10.5)$ & $18(8.7)$ & $69(25.0)$ \\
\hline Values & $6(4.0)$ & $4(1.4)$ & $8(5.3)$ & $21(7.6)$ & $4(2.7)$ & $25(10.5)$ \\
\hline Country & $8(5.3)$ & $39(14.1)$ & $5(3.3)$ & $11(4.0)$ & $4(2.7)$ & $11(4.0)$ \\
\hline Cure from illness & - & N/A & $0(0)$ & N/A & $17(11.3)$ & N/A \\
\hline Kidney transplant & - & N/A & $2(1.3)$ & N/A & $15(10.0)$ & N/A \\
\hline Living with illness & - & N/A & $1(0.7)$ & N/A & $8(5.3)$ & N/A \\
\hline
\end{tabular}

From the table above and when reading across the three questions, it can be seen that money was reported to be the most important determinant of QOL by dialysis respondents. There were $60.7 \%$ of the dialysis patients who personally value health in life but only $36.7 \%$ considered health as most important to them in maintaining or improving their QOL. A considerable number (40.7\%) indicated that people in UAE valued money most in life. Also, $24.7 \%$ of the dialysis patients personally valued money most in life and believed that money was a major determinant in improving and maintaining their QOL. One of the respondents responded that "if you have money you will get the best treatment". Another respondent commented that "Dirhams [local currency] make people appreciate you and will change your status in the society". Although more than one third (38.6\%) of the dialysis respondents indicated that people in UAE valued health as well, two thirds $(60.7 \%)$ of the respondents personally valued health most in life. Around one third (36.7\%) believed that health is most important in maintaining or improving their QOL. One of the respondents commented that "I wish if I have my health and not have the money". Half of the 
respondents from the dialysis sample valued family most in life and nearly a fifth (18.7\%) saw family support as a major determinant in improving and maintaining their QOL. Few respondents $(5.3 \%)$ believed that people of the UAE valued the country and its leadership most in life, while only $3 \%$ personally valued the country and its leadership most, and respondents reported that the country played an important factor in maintaining and improving their QOL.

Health was reported to be the most important determinant of QOL by the community sample. In that sample $44.9 \%$ of respondents reported that people in the UAE valued health most in life. Also, $66.1 \%$ of the community respondents personally valued health most in life. Additionally, $27.9 \%$ believed that health was a major determinant in improving and maintaining their quality of life. "Being healthy is a fortune in itself", a respondent commented. Around one third (31.2\%) of the respondents from the community sample believed that people in UAE value family as well. More than two thirds $(66.1 \%)$ of the respondents personally valued family most in life and a sixth indicated that family support was most important in maintaining or improving their QOL. Another major determinant of QOL in UAE was money; around half of the respondents indicated that the UAE population valued money most in life. Also, around one fifth of the respondents personally valued money most in life. Furthermore, 38.4\% of the respondents indicated that money was the most important factor in improving and maintaining their QOL.

\subsubsection{Comparison of the responses to open-ended questions for both samples}

This section reports on the findings from the qualitative data for both samples. Firstly, it presents the results of what the responders indicated others value most in life. Secondly, it presents the results of what the responders personally value most in life. Thirdly, it presents the results of what are the things that improve or maintain their QOL.

The first question explored what the people in UAE valued most in life. Comparisons of the themes identified by both samples are presented in Table 5.12. Around one third of the community sample indicated that people in UAE valued family most in life compared with $17.3 \%$ in the dialysis sample. Furthermore, approximately one quarter of the dialysis sample indicated that people in UAE valued safety in life compared with a sixth in the community sample. Also, $14.1 \%$ of the community sample indicated that people in UAE 
valued their country most in life compared with $5.3 \%$ in the dialysis sample. On the other hand, less than half of both samples (43.9\%) indicated that the UAE people valued money most in life. Furthermore, $42.7 \%$ indicated that UAE people valued health most in life. Also, more than one quarter (26.5\%) of both samples indicated that UAE people valued health most in life. Moreover, $11.0 \%$ indicated that UAE people valued country most in life and $18.8 \%$ indicated that UAE people valued safety most in life.

The second question explored what the respondents personally valued most in life. A comparison of the themes identified by the dialysis and the community samples are presented in Table 5.14. Around two thirds of the community sample personally valued family most in life compared with half in the dialysis sample. Moreover, $14 \%$ of the dialysis sample personally valued relationships compared with $6.5 \%$ in the community sample. Also, $29.3 \%$ of the respondents from both samples personally valued religion most in life. Around two thirds of the respondents from both samples valued health most in life. However, only a few respondents (9.2\%) from both samples personally valued education most in life. Furthermore, $3.8 \%$ of the respondents from both samples personally valued their country most in life.

The third question explored what things were most important to the respondents in maintaining or improving their quality of life. A comparison of the themes identified by the dialysis and the community samples is presented in Table 5.14. Less than one third $(31.0 \%)$ of the respondents from both samples indicated that health was important in maintaining and improving their QOL. Furthermore, around one fifth $(20.7 \%)$ of the respondents from both samples indicated that jobs were important in maintaining and improving their QOL. Moreover, $12.2 \%$ of the respondents from both samples indicated that religion was important in maintaining and improving their QOL. Additionally, almost one eighth $(17.4 \%)$ of the respondents from both samples indicated that family was important in maintaining and improving their QOL. Only 6.3\% of the respondents from both samples indicated that housing was important in maintaining and improving their QOL. Also, only $3.5 \%$ of the respondents from both samples indicated that their country was important in maintaining and improving their QOL. More than one third of the community sample indicated that money was important in maintaining and improving their QOL compared with almost one quarter of the dialysis sample. One quarter of the community 
sample indicated that jobs were important in maintaining and improving their QOL compared to one eight in the dialysis sample. Also, one quarter of the community sample indicated that an education was important in maintaining and improving their QOL compared with $8.7 \%$ of the dialysis sample. Furthermore, around one in 10 respondents from the community sample indicated that values were important in maintaining and improving their QOL compared with $2.7 \%$ in the dialysis sample.

\subsubsection{Themes ranking}

The identified themes have been ranked according to the number and percentages of respondents who chose them. The ranking order is summarised in Table 5.15.

Table 5.15 Comparison of themes ranking in both samples

\begin{tabular}{|c|c|c|c|c|c|c|}
\hline Themes & $\begin{array}{l}\text { Ranking } \\
\text { Question } \\
\text { 1 Dialysis }\end{array}$ & $\begin{array}{l}\text { Ranking } \\
\text { Question } 1 \\
\text { Community }\end{array}$ & $\begin{array}{l}\text { Ranking } \\
\text { Question } 2 \\
\text { Dialysis } \\
\end{array}$ & $\begin{array}{l}\text { Ranking } \\
\text { Question } 2 \\
\text { Community } \\
\end{array}$ & $\begin{array}{l}\text { Ranking } \\
\text { Question } 3 \\
\text { Dialysis } \\
\end{array}$ & $\begin{array}{l}\text { Ranking } \\
\text { Question } 3 \\
\text { Community }\end{array}$ \\
\hline Health & 2 & 2 & 1 & 1 & 1 & 2 \\
\hline Money & 1 & 1 & 4 & 3 & 2 & 1 \\
\hline Family & 6 & 3 & 2 & 1 & 4 & 6 \\
\hline Happy life & 3 & 4 & 5 & 4 & 3 & 5 \\
\hline Religion & 5 & 5 & 3 & 2 & 7 & 8 \\
\hline Safety & 4 & 6 & 6 & 5 & 6 & 4 \\
\hline Relationship & 9 & 9 & 7 & 8 & 5 & 7 \\
\hline Job & 7 & 8 & 8 & 5 & 8 & 3 \\
\hline Housing & 8 & 11 & 11 & 9 & 12 & 10 \\
\hline Education & 11 & 10 & 9 & 6 & 11 & 3 \\
\hline Values & 11 & 12 & 10 & 7 & 14 & 9 \\
\hline Country & 10 & 7 & 12 & 9 & 14 & 11 \\
\hline $\begin{array}{l}\text { Cure from } \\
\text { illness }\end{array}$ & - & - & $\mathrm{N} / \mathrm{A}$ & $\mathrm{N} / \mathrm{A}$ & 9 & $\mathrm{~N} / \mathrm{A}$ \\
\hline $\begin{array}{l}\text { Kidney } \\
\text { transplant }\end{array}$ & - & $\mathrm{N} / \mathrm{A}$ & 13 & $\mathrm{~N} / \mathrm{A}$ & 10 & $\mathrm{~N} / \mathrm{A}$ \\
\hline $\begin{array}{l}\text { Living with } \\
\text { illness }\end{array}$ & - & $\mathrm{N} / \mathrm{A}$ & 14 & $\mathrm{~N} / \mathrm{A}$ & 13 & $\mathrm{~N} / \mathrm{A}$ \\
\hline $\begin{array}{l}\text { Alternative } \\
\text { to dialysis }\end{array}$ & - & $\mathrm{N} / \mathrm{A}$ & 14 & $\mathrm{~N} / \mathrm{A}$ & - & $\mathrm{N} / \mathrm{A}$ \\
\hline Social life & - & - & 14 & - & - & - \\
\hline
\end{tabular}

Table 5.15 showed that the dialysis and community respondents ranked money as the most valued thing in life. The dialysis sample ranked a happy life as the third valuable thing that they believe other people in the UAE valued most in life. On the other hand, the 
community sample ranked family as the third thing that that they believed other people in UAE valued most in life. Safety was ranked as a fourth element by dialysis respondents while the community sample ranked a happy life as the fourth element that others valued most in life. Both samples ranked religion as the fifth valued thing in life by other people in UAE. Interestingly, the dialysis sample ranked family as the sixth valued element, whereas the community sample ranked safety as the sixth valued element in life.

The community sample ranked health and family as the first thing they personally valued most in life, while the dialysis sample ranked health first and family second. Religion was the second priority for the community sample and the third priority for dialysis sample. Money was ranked third for the community sample and was also ranked as the fourth option for the dialysis sample. Having a happy life was ranked as the fourth element in the community sample and as the fifth element in the dialysis sample.

The dialysis and community samples ranked the most important to them in maintaining or improving their QOL differently. As expected, health was ranked first by dialysis sample, while money was ranked first by the community sample. In contrast, money was ranked second by the dialysis sample and health was ranked second by the community sample. A happy life was ranked third by the dialysis sample while a job and education ranked third by the community sample. Family was ranked fourth by the dialysis sample and safety was ranked fourth by the community sample. Relationship was ranked fifth by the dialysis sample and a happy life was ranked fifth by the community sample. Further details on ranking orders are outlined in the above table.

\subsubsection{Themes representations in the QOL tools}

The analysis of the themes and the dimensions of the subscales from both tools found that some of the themes were not addressed by the QOL tools. Table 5.16 summarises the themes and its representations in both tools. 
Table 5.16 Representation of the themes in the SF-36 and QOL Index

\begin{tabular}{|c|c|c|}
\hline Themes & Level addressed in SF36 & Level addressed in QOL Index \\
\hline Health & Yes & $\begin{array}{ll} & \text { Yes }\end{array}$ \\
\hline Money & No & Partially represented \\
\hline Family & Partially represented & Yes \\
\hline Happy life & Yes & Yes \\
\hline Religion & No & Yes \\
\hline Safety & No & No \\
\hline Relationship & Yes & Yes \\
\hline Job & Yes & Yes \\
\hline Cure from illness & No & Yes \\
\hline Housing & No & Yes \\
\hline Education & No & Yes \\
\hline Kidney transplant & No & Yes \\
\hline Values & No & No \\
\hline Country & No & No \\
\hline Living with illness & No & Yes \\
\hline Alternative to dialysis & No & Yes \\
\hline Social life & Yes & Yes \\
\hline $\begin{array}{ll}\text { Total } & \text { Covered } \\
& \text { Partially covered } \\
& \text { Not Covered }\end{array}$ & $\begin{array}{r}5 \\
1 \\
11\end{array}$ & $\begin{array}{r}13 \\
1 \\
3\end{array}$ \\
\hline
\end{tabular}

Not all themes identified by the dialysis patients and the community samples were represented or addressed in the QOL tools. Furthermore, the number of themes differed between the SF-36 and QOL Index tools. Some of those themes were covered, partially covered or not covered. Religion, cure from illness, housing, education, kidney transplant, living with illness and an alternative to dialysis were covered in the QOL Index but not in the SF-36. On the other hand, safety, values and country were not covered by either tool. Family was partially addressed in the SF-36 and fully addressed in the QOL Index. Money was partially addressed in the QOL Index and was not addressed in the SF-36.

\subsection{Conclusion}

The return rate for the dialysis and community samples was $93 \%$ and $76 \%$ respectively. More questions were missed by respondents from both samples when using the QOL Index. The total numbers of missing data were statistically insignificant and did not have an impact on the data analysis. There are statistically significant differences in all the demographic or independent variables between the two samples. Males were dominant in both samples, but they were more dominant in the dialysis sample. The dialysis sample was older by 8.6 years. The numbers of married respondents in both samples were almost 
the same. The vast majority of the community sample had full-time employment. More than half of the dialysis sample suffered from chronic illnesses other than kidney failure, whereas only one eighth of respondents from the community sample had on-going chronic illness. The mean and median, skewness and kurtosis, normality plots, Q-Q plot, extreme values and outliers test showed that the data were normally distributed. In contrast, kolmogorov-smirnov $Z$ test showed that the data were not normally distributed. Given that none of the subscale components of the two tools were directly comparable, a comparison of scores of the two instruments could only be made on the total scores.

Both tools were considered culturally relevant by the vast majority of the dialysis and community respondents. Of particular importance, four dialysis patients and six community respondents considered neither tool culturally relevant. The analysis of the qualitative data revealed that health, family and money were the major themes identified in determining the QOL in both samples. Around one third of the community sample believed that people in UAE valued family most in life compared with almost one eighth in the dialysis sample. Nearly less than half of both samples believed that UAE people valued money most in life. Around two thirds of the community sample personally valued family most in life compared with $60 \%$ in the dialysis sample. Both samples valued other things that are not addressed in the tools, such as safety, values and religion. The samples ranked the themes differently. 


\section{Chapter 6 Discussion on sample characteristics and cultural relevancy}

This chapter is the first of two chapters that discuss the findings of this study. This chapter focuses on the sample characteristics and cultural relevancy of the tools. The first section discusses the return rates. Section two discusses the characteristics, representation, and the demographic similarities and differences of both samples. Section three discusses the cultural relevancy of the tools and examines the impact of missing data in relation to cultural relevancy. Moreover, this section explores the characteristics of the respondents who did not answer questions that had the highest rate of missing data to establish whether there is a cultural element to this. Section four discusses the usage of the QOL tools in clinical settings. The last section discusses the findings from the qualitative data and compares the responses to the open-ended questions between both samples. Furthermore this section also explores the themes that were identified by the respondents when answering the open-ended questions and examines their ranking.

\subsection{Return rates}

The return rate in this study of $93 \%$ for the dialysis sample is high compared with Sorensen et al.'s (2007) study on the prevalence of complications, health-related QOL and the influence of beliefs about how people with diabeties on dialysis control their health. Sorensen et al. had a return rate of $76 \%$. Suet-Ching (2001) had a similar response rate to this UAE study of $91 \%$ when she studied the QOL in a group of dialysis patients from Hong Kong. The high return rate in this study could be attributed to four reasons. Firstly, this research was conducted in one centre in the UAE where the dialysis sample was obvious and easy to contact and follow up, especially answering the participants' questions regarding the QOL tools and the nature of study. Secondly, the majority of the respondents knew the researcher through his work as a clinical resource nurse. The researcher's role was mainly educating both patients and nurses on dialysis related topics. Lastly, knowing the researcher prior to conducting the research and his good relationship with potential respondents might have influenced people's decisions to participate. Thirdly, the respondents were provided a return envelope as recommended by Edwards et al. (2002). Fourthly, the respondents were contacted by the recruiting nurse before giving them the survey package. The face-to-face contact showed that the researcher cared about the 
respondents, which is likely to have an influence on the return rate. However, dialysis patients were not obligated to participate in the study. As outlined in the methodology chapter all patients signed a consent form (Appendix 2) and it was highlighted in the accompanying invitation letter (Appendix 1) that their care would not be affected whether they participated in the study or not.

The dialysis sample size was relatively small compared with some other studies, in a study on 1047 Russian hemodialysis patients (Vasilieva, 2006), 861 dialysis patients participants from Taiwan (Kao et al., 2009) and 705 dialysis patients in the United Kingdom (Gudex, 1995). On the other hand, it is large compared with Acaray and Pinar (2005) sample of 100 Turkish hemodialysis patients and Morsch, Gonçalves and Barros (2006) study of 48 hemodialysis patients in Brazil. The return rate of $76 \%$ for the community sample was relatively high when compared with some community samples, such as one by Covic et al. (2004) who had a return rate of $50 \%$ when comparing 82 clinically stable hemodialysis patients who completed the SF-36 tool in Romania with 1192 Romanian respondents. In contrast, the community sample in this study is relatively small compared with studies on 2114 Russian respondents (Vasilieva, 2006) and 4080 respondents from Denmark (Molsted, Prescott, Heaf, \& Eidemak, 2007), 2000 from the Irish general population (Cleary, 2005).

\subsection{Demographic and clinical data of both samples}

\subsubsection{Sample characteristics}

This is the first study reported on the QOL of the UAE dialysis population. There were 12 dialysis centres in the UAE. The dialysis sample was taken from the largest dialysis centre. The filters, blood lines and other equipment used vary from one centre to another. It is the researcher's view that the quality of care provided to dialysis patients in the study centre is comparable with other dialysis centres in the country. It is likely, however, that the standard of living of this sample might differ from other dialysis patients in the UAE. UAE National dialysis patients constituted $28 \%$ of the dialysis sample and they were eligible to receive free medical treatment, financial aid and other kinds of social assistance from the government. In contrast, other ethnic groups who live in the country were not entitled to receive any aid from the government. Furthermore, 10 respondents from South East Asia 
were unable to speak or write in English or Arabic and the assigned nurses translated the questions and answers to them. Answers given may not reflect the true situation; assisting patients to complete the tools may have had an impact on how people responded. This could be one of the limitations of this study.

The community sample was chosen from a group of the general population and sourced from different areas in Abu Dhabi. The researcher aimed to recruit people from different social classes, professions and backgrounds. However, the collected data on demographic variables indicates this did not occur. As people were recruited at random from different public areas and the researcher had no previous knowledge about their demographic characteristics. Therefore, there may be respondents who belong to different backgrounds that did not return the questionnaires. Also, the selected public areas might not have respondents from all different backgrounds.

\subsubsection{Sample representation}

It is important to reflect on how the two samples differ to enable a discussion of the possible impacts of the independent variable on the QOL for both samples. Each of the samples was selected differently. The dialysis sample was selected from one dialysis centre in the UAE. The community sample was an opportunistic sample, with respondents chosen at random from public places. Establishing the representativeness of the community sample was not possible for three reasons. Firstly, the UAE is composed of seven cities; the standards of living in each city are different. Abu Dhabi and Dubai are the richest cities and the standard of living was and still very high compared with relatively poor cities such as Fujairah and Umm al-Quwain. Given that people in the UAE generally live and work in the same city it is presumed that all community respondents were residents of Abu Dhabi. Secondly, the general culture in the country may look the same, but there are subcultures and some of them are more conservative than others (ElHaddad, 2006). Thirdly, different parts of the UAE may have different values. Urban populations and rural populations may think differently (Dao-qi, 1990). Lastly, women in Abu Dhabi and Dubai are more liberal compared with women in Fujairah and Umm alQuwain (Women in the UAE, 2007). This difference may be because most expatriates in UAE reside in Abu Dhabi and Dubai, which means that women in these two cities are more likely to have interacted with people from other cultures. 
The ethnicity of the dialysis sample differed in comparison with the general population of the UAE, in that there were more UAE Nationals in the dialysis sample compared with UAE population ( $28 \%$ vs $11 \%$ ), more other Arabs ( $38 \%$ vs $21 \%$ ), and fewer South East Asians (23\% vs $57 \%$ ). The ethnicity of the community sample differed in comparison with the population of the UAE, in that there were slightly more UAE Nationals (12.0\% vs $11 \%)$, more other Arabs (50.9\% vs 21\%), and fewer South East Asians (23.6\% vs 57\%) (Pejman, 2007). In summary, the community sample is not representative of the UAE adult population. This could be considered a limitation of this study. However, given that there are no studies published on the UAE population, the findings from this study remain valuable.

\subsubsection{The demographic similarities and differences of the samples}

The similarities and differences of the samples were summarised in the previous Chapter The majority of the community sample was more educated and had full-time employment than the dialysis sample, where less than a quarter had a tertiary education and around one third had a full-time employment. The level of education in most countries is linked to employment prospects and income (Molsted et al., 2004). A considerable number of dialysis patients do not work due to the nature of the disease which requires longer hours on dialysis. Part-time work is not a common practice in the Middle East, where most employment is full-time. The findings also indicated that the dialysis sample was generally older than the community sample.

The dialysis sample had generally resided longer in the UAE and had not travelled outside of the UAE as recently as most of the community sample. These differences are likely to be due to the restrictions of the disease, and the need for dialysis treatment during travelling to other countries. Furthermore, several patients from South East Asia reported to the researcher while receiving dialysis that they did not have free access to dialysis treatment in their home countries. Therefore, they stayed in the UAE to receive free treatment. The majority of both samples were married but the number of divorced respondents from the dialysis sample was higher than the community sample. This could be attributed to the impact of dialysis treatment on marriage. People on dialysis are known to have altered body image (Beer, 1995) and given their health demand often have to 
change roles in the family (Belasco et al., 2006). The impacts of these differences in the demographic variables between the samples will be discussed in the next chapter.

\subsection{Cultural relevancy of the tools}

It was shown that both tools were considered culturally relevant by the majority of respondents in both samples. When stating an actual preference as to which tool was relevant approximately a third (31\%) of the dialysis sample chose the QOL Index while fewer than a fifth (17\%) chose the SF-36. The QOL Index was probably preferred more than the SF-36 because the QOL index has a dialysis version which has questions specifically related to the dialysis treatment and its implications on their life, unlike the SF 36 which is a general tool. In addition, the QOL Index has spiritual and family subscale that measures the satisfaction with and importance of certain elements related to religion and family issues. Having these subscales might have meant respondents felt that the QOL Index addresses vital issues related to their culture and life in general, unlike the SF-36 that measures the health status and the degree of disability.

The percentage of respondents from the community sample who considered the SF-36 and the QOL Index not culturally relevant almost double in the number in the dialysis sample (5.3 vs. 9.4 respectively). This could be related to the fact that respondents from the community sample were more educated and younger in comparison with the dialysis respondents. People at a younger age look at life and interpret life issues differently. Further analysis of the demographic characteristics of the respondents who considered that the QOL tools were not culturally relevant shown in Table 5.11 revealed that the highest percentage of the respondents who considered both tools were culturally not relevant were married. Furthermore, the percentage of married respondents from the dialysis sample who considered the SF-36 (76\%) and the QOL Index (43\%) not culturally relevant were lower than the percentage of respondents from the community sample who considered the SF-36 (84\%) and QOL Index (77\%) not culturally relevant. There are no readily available explanations for these findings.

Table 5.11 also revealed that the highest percentage of the respondents who considered both tools were culturally not relevant belonged to the South East Asian ethnicity compared with other ethnic groups. Furthermore, the percentage of the South East Asian 
respondents from the dialysis sample who considered the SF-36 (38\%) and QOL Index $(43 \%)$ not culturally relevant were almost similar to the percentage of respondents from the community sample who considered the SF-36 (36\%) and the QOL Index (46\%) not culturally relevant. This finding supports the previous argument that people from different cultures perceive QOL differently. Furthermore, the QOL of the South East Asian ethnicity should be investigated separately preferably in their home countries first, and then a comparison can be made by comparing the QOL between the people who live in South East Asian countries and the South East Asians who live in the UAE or other countries.

The majority of respondents in the dialysis sample, who considered that the SF-36 was not culturally relevant, belonged to Muslim (38\%) and Christian (38\%) religions and 50\% of them had attended primary schools. Similar findings were noticed in the QOL Index tool, the majority of respondents in the dialysis sample, who considered that the QOL Index was not culturally relevant, belonged to Muslim (43\%) and Christian (43\%) religions, but $44 \%$ of them had attended secondary schools. While in the community sample the majority of respondents, who considered that the SF-36 and the QOL Index tools were not culturally relevant, belonged to others religion (52\%) in SF-36, Christian 60\% in QOL Index, had tertiary education (48\%, 23\% respectively) and attended secondary schools (44\%, 77\% respectively). There were no questions that talk about religious beliefs in the SF-36 tool. In contrast, there were two questions about the satisfaction and importance of living as they would like, and two questions about satisfaction and importance of their faith in God.

The culture and the different interpretation of the questions are likely to influence how respondents understand questions dealing with QOL (Angel \& Cronfein, 1988). Cultural mediation and cultural experience influences the meaning or validity of the questions. When the respondent replies, the reply is not to the same question that has been asked; hence, it is not valid (Angel \& Thoits, 1987). Cognitive assessment seeks to understand what the question means to the respondent and, if the meaning is different from that intended by the questioner, to guide the choice of more culturally or educationally appropriate wording (Angel \& Thoits). Also, cultural background may affect adaptation to chronic hemodialysis therapy (Weisbord et al., 2008) and consequently affect their QOL. 
The QOL tools used in this research are universal tools; the effect of category fallacy phenomenon should not be ignored. Category fallacy results from the failure to distinguish between the concepts that are truly universal and accepted across multiple culture groups and the concepts that have meaning only within a specific cultural group or socioeconomic context (Warnecke et al., 1996). Furthermore, sometimes the language into which a question is translated does not contain the right concept (Angel \& Thoits, 1987). For example, in the SF-36, question 23 asked respondents "Did you feel full of pep?" This question may not have a clear universal meaning to people from different cultures especially the UAE. Moreover, the UAE culture and the interpretation differences of the questions by the UAE population might influence how respondents understand questions related to QOL.

Responding to questions mainly depends on how people retrieve information from their memory. Respondents' responses are mainly influenced by response editing. Response editing is a commonly encountered phenomenon when survey respondents feel that certain answers are more socially desirables than others (Warnecke et al., 1996). Culture, religion and family have special influence on people from the UAE. Therefore, questions related to religion and family in both tools may come under the response editing category, where normally satisfaction and importance of religion are taken for granted among Muslim people.

While Table 5.10 indicated that $4(2.7 \%)$ of the dialysis sample and $6(2.2 \%)$ in the community sample considered that neither tool was culturally relevant, these numbers are low given the focus of this research an examination of the data was undertaken to see whether there were any shared characteristics amongst these individuals. The demographic characteristics for those respondents who considered that neither tool was culturally relevant may also draw some light on the cultural relevancy and acceptability of the tools.

Of the four respondents from the dialysis sample who considered neither tool was culturally relevant, only one respondent did not answer the question that asked about the satisfaction and the importance of sex to them. In contrast, of the six respondents from the community sample who considered neither tool was culturally relevant, only one 
respondent did not answer the question that asked about the satisfaction and the importance of sex to them. Questions about satisfaction and importance of sex assumed that the individuals have stored in their memories relevant experiences that will be available for forming judgments about the importance of and current satisfaction with this aspect of their life. If there are no memories of sex and relevant events to cue the patient's responses, the actual responses to questions about how much the patient is satisfied with sex aspect may be based on motivation to be a "good respondent". Hence the responses may be subject to editing rather than reflecting the respondent's true assessment (Warnecke et al., 1996).

\subsubsection{Missing data}

As per Table 5.7, the importance and satisfaction of children and spouse, lover, or partner items in the family subscale had highest proportions of missing data. This may indicate that these questions were not applicable or not appropriate to them because they were not married or did not have children. So, the majority of the missing data came from the health and functioning and family subscales. It is worth highlighting that the higher percentage in the missing data for questions 22 and 23 that asks respondents to state their opinions about their satisfaction and importance of their job or not having a job is due to respondents needing to answer only one question of the two. However, 12 respondents answered both and another 12 respondents missed both questions. The 12 respondents who missed both questions fitted no demographic profile except that the majority of them were not employed. This indicates that having this paired option was confusing for some respondents.

Table 5.8 presented the missing data in relation to subscales of QOL Index for community sample. This table showed similar findings to the dialysis sample, the satisfaction and importance of chances for living as long as they would like and sex life items in the health and functioning subscale for the community sample had the highest proportions of missing data $(11.2 \%, 10.5 \%$ and $13.9 \%, 10.9 \%$ respectively). The reason for these findings is related to religious and cultural beliefs that nobody is having control on how many years they are going to live for. The high percentage for not completing the question about the satisfaction with and importance of sex life is mainly related to religious believes and cultural norms that single people were not allowed having sex before marriage. Of the 
community sample $10.5 \%$ did not answer the question about their satisfaction with their body pain. Furthermore, the importance and satisfaction of children (16.5\%, 11.6\%) and spouse, lover, or partner $(13.9 \%, 9.4 \%)$ items in the family subscale had the highest proportions of missing data. Similar to the dialysis sample, the majority of the missing data came from the health and functioning and family subscales.

The finding that only $9(6 \%)$ of the dialysis respondents did not answer the question about their satisfaction with their chances of living as long as they would like, compared with 30 $(11 \%)$ from the community sample and 8 (5\%) of the dialysis respondents did not answer the question about the importance of living as long as they would like, compared with more than double the percentage $28(11 \%)$ in the community sample could be related to the fact that Muslim people believe that nobody has control over how long he/she is going to live for. Dialysis patients in general are aware of imminent death due to the complications of their disease. This might indicate that they do not want to talk about whether they are satisfied with how long they are going to live.

Of the nine respondents from the dialysis sample who did not answer the question about their satisfaction with their chances of living as long as they would like to, eight of them were Muslims and one Christian. Similarly, of the 30 respondents who did not answer the same question from the community sample, there were 29 Muslims and one Christian. With regards to the missing responses from the question that asked about the importance of living as long as they would like to, all of the eight respondents from the dialysis sample were Muslims. In contrast, of the 28 respondents from the community sample who did not answer the same question there were 25 Muslims and three Christian. Reasons for not answering these two questions might be the appropriateness of the questions from the cultural and the religious point of view of the respondents. It was highlighted in the conceptual framework that in Islamic culture, nobody has control on how long they are going to live for. Muslims believe that life, death and illnesses come from God and nobody can predict how long he/she is going to live for. "Lo! Allah! With Him is knowledge of the Hour. He sends down the rain, and knows that which is in the wombs. No soul knows what it will earn to-morrow, and no soul knows in what land it will die. Lo! Allah is Knower, Aware (34) (Luqman 31:33). 
Furthermore, slightly more respondents from the dialysis sample 24 (16\%) did not answer the question about their satisfaction with their sex life compared with 37 (14\%) from the community sample and, 21 (14\%) of the dialysis sample did not answer the question about the importance of sex to them compared with $29(11 \%)$ from the community sample. Ali et al. (2005) reported that the prevalence of erectile dysfunction among 75 hemodialysis patients in Egypt was $82.5 \%$. There were $25(17 \%)$ of the dialysis patients who did not answer the question about their satisfaction with their partners, spouse or lover compared with $37(14 \%)$ of respondents from the community sample. Among these 15 were single and 22 were widows. Those respondents were not married and did not have partners. Therefore, these questions might be in appropriate or not applicable to them. Having a partner or lover is prohibited in the Islamic countries and Muslims are not allowed to have any kind of sexual relationship before marriage.

There were no gender and marital status differences in the missing data regarding satisfaction and importance of spouse/partners between males and females for both samples. More Muslim dialysis patient did not answer the questions about their satisfaction and importance of spouse/partners to them compared with Christians and people from other religions. Furthermore, more single and Muslim respondents from the community sample did not answer the questions about the satisfaction with and the importance of spouse/partners to them. The likely reason for this is they were not married therefore would not have had spouses or lovers. Edwards et al. (2002) reported that questionnaires containing questions of a sensitive nature such as sex life were less likely to be returned or answered. It is worth highlighting that neither tool had a non-applicability option. Therefore respondents missed these questions.

\subsubsection{Comparison of the characteristics of the respondents who did not answer the questions about satisfaction with and importance of sex and spouse for both groups}

A summary of findings about characteristics of the respondents who did not answer the question about satisfaction with and importance of sex and spouse for both samples is presented in Table 6.1. From this table it is apparent that slightly more dialysis female respondents did not answer questions about their satisfaction and importance of sex than male dialysis respondents. In contrast, the number of female respondents from the 
community sample who did not answer these questions was almost double the number of the male respondents. The majority of the non-respondents to this question in the community sample were single. In contrast, the majority of non-respondents in the dialysis sample were married. Recalling information about regular recurring events is likely to be culturally influenced by the individual's community or larger culture (Angel \& Lumpkin, 1992). Also, as it was explained earlier, Muslim people are not allowed to have sex before marriage, so they probably have had no sexual experiences before. There is no stored information or experiences to retrieve about sexual intercourse from their memories; therefore they did not answer those questions about satisfaction and importance of sex. Some of the missed questions were not applicable to some of the respondents such as satisfaction with partners or children if they were not married or they did not have children, or satisfaction with their sex life if they were single. This could explain why some respondents did not answer this question. However, declining to share any information about sex life could be attributed to cultural and social reasons in terms of how families raise their children and the values and norms they teach them. 
Table 6.1 Comparison of the characteristics of the respondents who did not answer the question about satisfaction with and importance of sex and spouse for both samples

\begin{tabular}{|c|c|c|c|c|c|c|c|c|c|}
\hline Variables & & $\begin{array}{c}\text { Dialysis } \\
\text { sample } \\
\text { Satisfaction } \\
\text { with sex } \\
n(\%)\end{array}$ & $\begin{array}{c}\text { Community } \\
\text { sample } \\
\text { Satisfaction } \\
\text { with sex } \\
\text { n(\%) }\end{array}$ & $\begin{array}{c}\text { Dialysis } \\
\text { sample } \\
\text { Importance } \\
\text { of sex } n(\%)\end{array}$ & $\begin{array}{l}\text { Community } \\
\text { sample } \\
\text { Importance } \\
\text { of sex } n(\%)\end{array}$ & $\begin{array}{c}\text { Dialysis } \\
\text { sample } \\
\text { Satisfaction } \\
\text { with } \\
\text { spouse } \\
\mathrm{n}(\%)\end{array}$ & $\begin{array}{l}\text { Community } \\
\text { sample } \\
\text { Satisfaction } \\
\text { with } \\
\text { spouse } \\
n(\%)\end{array}$ & $\begin{array}{c}\text { Dialysis } \\
\text { sample } \\
\text { Importance } \\
\text { of spouse } \\
\mathrm{n}(\%)\end{array}$ & $\begin{array}{c}\text { Community } \\
\text { sample } \\
\text { Importance } \\
\text { of spouse } \\
n(\%)\end{array}$ \\
\hline \multirow[t]{2}{*}{ Gender } & Male & $10(6.7)$ & $13(4.9)$ & $8(5.3)$ & $10(3.7)$ & $11(7.3)$ & $18(6.7)$ & $9(6.0)$ & $9(3.4)$ \\
\hline & Female & $14(9.3)$ & $24(9.0)$ & $13(8.7)$ & $19(7.1)$ & $14(9.3)$ & $19(7.1)$ & $8(5.3)$ & $16(6.0)$ \\
\hline \multirow{4}{*}{$\begin{array}{l}\text { Marital } \\
\text { status }\end{array}$} & Single & $8(5.3)$ & $25(9.4)$ & $5(3.3)$ & $22(8.2)$ & $12(8.0)$ & $29(10.9)$ & $5(3.3)$ & $18(6.7)$ \\
\hline & Married & $9(6.0)$ & 7 (2.6) & $7(4.7)$ & $5(1.9)$ & $3(2.0)$ & $3(1.1)$ & $3(2.0)$ & $5(1.9)$ \\
\hline & Divorced & $4(2.7)$ & $3(1.1)$ & 5 (3.3) & $1(0.4)$ & $5(3.3)$ & $3(1.1)$ & $4(2.7)$ & $1(0.4)$ \\
\hline & Widows & $3(2.0)$ & $2(0.7)$ & $8(5.3)$ & $1(0.4)$ & 5 (3.3) & $2(0.7)$ & $5(3.3)$ & $1(0.4)$ \\
\hline \multirow[t]{3}{*}{ Religion } & Muslims & $20(13.3)$ & $29(10.9)$ & $18(12.0)$ & $21(7.9)$ & $19(12.7)$ & $28(10.5)$ & $15(10.0)$ & $19(7.1)$ \\
\hline & Christian & $4(2.7)$ & $6(2.2)$ & $2(1.3)$ & $6(2.2)$ & $5(3.3)$ & $8(3.0)$ & $2(1.3)$ & $6(2.2)$ \\
\hline & $\begin{array}{l}\text { Other } \\
\text { religions }\end{array}$ & $0(0.0)$ & $2(0.7)$ & $1(0.7)$ & $2(0.8)$ & $1(0.7)$ & $1(0.7)$ & $0(0.0)$ & $0(0.0)$ \\
\hline
\end{tabular}


More Muslim respondents 20 (13.3\%) from the dialysis sample did not answer questions about their satisfaction and importance of sex than Christians $4(2.7 \%)$ and people from other religions in both samples. By looking at the above figures from a different angle one finds that Christians constitute $16(10.7 \%)$ of the dialysis sample. The four Christian dialysis respondents who did not answer the question about their satisfaction with their sex life constitute $25 \%$ of the Christian dialysis sample and the 20 Muslim respondents who did not answer this question constitutes of $16.3 \%$ of the Muslim dialysis sample. Furthermore, there were $18(12 \%)$ Muslims and $2(1.3 \%)$ Christian dialysis patients who declined to share any information about the importance of sex to them. The two Christian respondents constitute $12.5 \%$ of the Christian dialysis respondents and the 18 Muslim respondents who did not answer this question constitute of $14.6 \%$ of the Muslim dialysis sample. In other words, slightly more Muslim dialysis respondents declined to share any information about the importance of their sex life to them than Christian dialysis respondents. The five Christian respondents constituted $31.3 \%$ of the Christian dialysis respondents and the 18 Muslim respondents who did not answer this question constitutes $15.4 \%$ of the Muslim dialysis sample. In other words, the percentage of Christian dialysis respondents who declined to provide any information about their satisfaction with their partners, spouse or lover is high compared with Muslim dialysis respondents. Furthermore, Muslims constituted $123(82 \%)$ of the dialysis sample. Also, Muslims constituted $180(67.4 \%)$ of the community sample. So, the majority of the respondents in both samples were Muslims.

The remarkable difference in the sample size between the number of Muslim, Christian, and respondents who belonged to other religions makes it difficult to compare and contrast the differences and draw conclusions because of the smaller sample size. In general, the percentages of the missing data in the dialysis sample were higher than the community sample. This could show that the dialysis sample was more conservative than the community sample, keeping in mind that the community sample was relatively younger and more educated than the dialysis sample. Given that the majority of Muslim respondents considered that both tools were culturally relevant both tools were culturally relevant to UAE population. However, the percentage of missing data in the QOL Index tool could be less if some of the questions were culturally relevant. 


\subsubsection{Respondents views on questions added or deleted from QOL tools}

Respondents from both groups suggested adding questions to both tool about the quality and satisfaction with the healthcare services provided, religion and spiritual values, income and financial status, satisfaction with and access to the healthcare, and relationship with healthcare team. The dialysis respondents suggested adding questions to both tools about the effect of dialysis on their daily life and the possibilities of having kidney transplant, coping with the disease and life stressors and description of feelings to be on dialysis. The community respondents suggested adding questions to both tools about the importance of eating habits and life style, family dynamics and support, the impact of the marital relationship on physical and mental health, questions about why people migrate to other countries, awareness of health status and adaptation to different cultures.

Six respondents from both samples suggested adding questions about life expenses in Abu Dhabi. Poor health condition puts an extra pressure on the income of the population and impacts negatively on the QOL of those with a limited income. Living in UAE is very expensive. Children of the expatriate population only attend costly private schools as they are not allowed to go to the government schools. Families who have patients with long term condition and two or more children at school may suffer financially as the expatriate patients need to pay for their medical treatment or pay for an expensive medical insurance. This can impact negatively on the QOL of patients and their families. Furthermore, expatriates need to pay for the medical insurance which is very expensive for people with long term conditions. So, having sufficient income to pay for all expenses is very important for all expatriates. As mentioned earlier $62.7 \%$ of the dialysis respondents were not employed. In the absence of a well established social security system that support sick individuals in the UAE, patients may suffer because they have insufficient income to pay the for medical treatment and maintain a reasonable QOL. Furthermore, house rents are very high and house owners usually increase the rent by $5 \%$ each year. This adds an extra pressure on the budget of the expatriates living in Abu Dhabi forcing a lot of poor dialysis patients to live in overcrowded poorly ventilated houses. The poor living conditions impacts negatively on their QOL (Kruger et al., 2006). One respondent from the community sample suggested a solution to the increase in the living expenses that government should stop the agents from increasing the house rent. 
The suggested added questions by the dialysis sample to the SF-36 mainly covered issues on diet, sexual life, kidney diseases and description of feelings while connected to the dialysis machine. For example respondents suggested questions about of health education about renal disease and coping with the disease. Some also suggested adding questions about their physical, social and emotional wellbeing. The suggested added questions by the community sample to the SF-36 covered issues on the effect of long term conditions on the caregivers, the rights of men and women, life expenses, the necessity of having free medical treatment for patients with long term conditions, importance of having sufficient income, the relationship between accomplishing personal goals and the improvement of the health status, the relationship between stress at work and the QOL, effect of the technology on health, loyalty to the country and level of activity and the effect of diet and eating habits on health status. Others suggested adding questions about the hot weather and its effect on the health and activity levels of patients with long term conditions. Gulf countries have relatively high temperature during summer reaching up to 50 degrees centigrade. The impact of high weather temperatures on the physical and psychological health is a topic that needs further investigations. Moreover, the impact of technology on the level of activity and physical health is another area that needs further research in the Middle East.

Respondents from the dialysis sample suggested adding questions to the QOL Index about the importance of taking their medication and the impact of doing regular physical activities on the QOL. The respondents from the community sample suggested adding more questions to the QOL Index about the importance of improvement in work conditions, future plans, ability of the respondents from other countries to cope with living in UAE and their abilities to cope when they return back to their home countries. The QOL Index measures the importance and the satisfaction with job, but it did not measure job security and the importance of professional development at work. Furthermore, it did not measure the importance of improvement in work conditions and achieving future plans on the QOL of employed people. Furthermore, both tools did not measure how people adapt to different cultures and the effectiveness of this adaptation. Measuring how people adapt to a new culture is very helpful in reducing the onsets of culture shock among people living in different countries (Yabroff, Linas, \& Schulman, 1996). 
None of the respondents from either samples suggested adding questions about sleep disturbances. The prevalence of insomnia is high in dialysis patients and has been reported to range from $45 \%$ to $59 \%$ (Iliescu, Yeates, \& Holland, 2004; lliescu et al., 2003; Sabbatini et al., 2002). Insomnia is associated with a substantial impairment in QOL (Roth, 2007). It may cause personal distress and adverse social and economic consequences, leading to a number of deleterious effects on behavior, health, sense of well-being, and enjoyment of interpersonal relationships (Roth; Roth \& Roehrs, 2003). Severe insomnia can impair daytime functioning, increase the occurrence of accidents and decrease QOL (Roth; Pai et al., 2007).

The comment that the SF-36 refers to experiences of the last four weeks only is also true. Most of the people with long term conditions suffered from their disease for more than four weeks and had suffered from tremendous amount of bad expediencies throughout the years. SF-36 limits the measurement of QOL to four weeks prior the time of completing the survey. So, the experiences prior to four weeks were not captured in this survey. This could be considered as one of the limitations of this tool. Only one community respondent commented that the questions of both surveys do not match the culture and the environment in the UAE and these tools may match the lifestyle and way of life for people from European countries. Respondents who answer any QOL tools need to feel that these tools were relevant to them and they need to have a feeling that these tools measure what they experience in daily basis. Some respondents may still answer them and may miss questions that they feel they were totally irrelevant. The last comment about people who complete anonymous surveys may hide that they suffer from long term conditions fearing that if they tell the truth they may lose their jobs is also true. In UAE the employment market is highly competitive and companies normally seeks to employ highly productive employees and avoid employing people with long term conditions. So, patients with long term conditions may hide their disease to keep their job. There were no sickness benefits from the governments in the Middle East. So people tend to hide their sickness even in anonymous surveys fearing that their employers may know about their illnesses.

Two respondents from the community sample suggested deleting questions 33, 34 and 35 from the SF-36 tool. These three questions measure general health. Questions 33 and 34 
requested the respondents to evaluate their health compared with other people "I seem to get sick a little easier than other people" and "I am as healthy as anybody I know". Question 35 asked the respondents to decide if they expect their health to get worse. People normally avoid losing hope about their medical condition. It is also part of their belief that nobody can decide or predict what will happen in the future. This may explain why these two respondents suggested deleting these questions.

One dialysis respondent suggested deleting question 7 "satisfaction and importance of the amount of control you have over your life?" Another suggested deleting question 8 "satisfaction and importance of your chances of living as long as you would like?" from the QOL Index. And a third suggested deleting the question 9 "satisfaction and importance of your faith in God? " A fourth respondent suggested deleting all questions that have well known answers but did not specify these questions.

Of the 10 respondents who suggested deleting questions from the QOL index four respondent suggested deleting question 7 "Your chances of living as long as you would like?' As explained earlier in Islamic culture nobody have control on how long are they going to live for except God. Three respondents suggested deleting question 11 "satisfaction and importance of sex life". As explained earlier in the conceptual framework, people from the Middle East are reluctant to display any information about their sex life except for medical reasons. One respondent from the community sample suggested deleting question 28 "satisfaction and importance of your faith in God" and question 33 "satisfaction and importance of yourself in general". Another respondent suggested deleting question 19 "satisfaction and importance of neighbours", question 23 "satisfaction and importance of your education". One respondent suggested deleting question 29 "satisfaction and importance of meeting your goal in life". Two respondents suggested deleting question 32 "satisfaction and importance of personal appearance" and question 33 "satisfaction and importance of yourself'. One respondent suggested deleting question 26 "satisfaction and importance of to having a happy future". One respondent suggested deleting question 3 "satisfaction and importance of the amount of pain that you have", question 18 "satisfaction and importance of the amount of worries in your life" and question 22 "satisfaction and importance of not having a job (if unemployed, retired, or disabled)". 
There were no explanations on why these respondents suggested deleting these questions.

The finding that some of the respondents suggested deleting or adding a few questions to the tools is similar to the findings of other researchers who have recommended adding or deleting questions from the tools to make them suitable to the culture of the respondents. For example Giacaman et al. (2009) who assessed the QOL in Palestinians living in the occupied Palestinian territory using the WHO QOL-Brief 88 tool, added some questions relevant to the Palestinian context, but the content of these were not detailed in their paper. Furthermore, during the cross-cultural adaptation and translation of the QOL measurement scales based on the example of St-George Respiratory Questionnaire, El Razike et al. (2009) modified the question about sports that did not concern women to adapt the original questionnaire to the Moroccan culture.

In the literature Awad, Denic and El-Tajic (2008) validated the European Organization for Research and Treatment of Cancer QOL Questionnaire for Arabic-speaking populations in the UAE. Due to cultural considerations, only married women were asked about sexual function. They found that the questions related to sexual function were acceptable to married respondents. A similar observation was made in the validation of the QLQ-BR23 questionnaire in Iranian married women lately. This latter group share many cultural characteristics with Arab patients (Pakpour et al., 2010). The QLQ-BR23 questionnaire is a disease-specific questionnaire, designed for patients with breast cancer (Montazeri et al., 2000).

QOL tools developed in other cultures should not only be tested for validity and reliability but also for cultural relevancy before using them with people from other ethnic or cultural backgrounds. Testing for cultural relevancy will ensure that minimal questions will be missed and all aspects of the QOL will be captured. Moreover, respondents will feel culturally safe and not offended. Since no other research studies have discussed the cultural applicability or relevancy of the QOL tools in dialysis patients, this is the first study to discuss the cultural relevancy of two QOL tools developed in western countries. 
In this study, the response rate could be higher and the level of missing data could be lower if all questions in the QOL Index tool were culturally relevant. For all the questions in the QOL Index to be culturally relevant the following changes are suggested: Firstly redesign the tools layout to have a section on marital status (e.g Are you married or single? If married, answer questions $10,11,12$ and 13. If single go to question 14 directly) or add a non-applicable section. Secondly, delete the questions about satisfaction and importance of chances of living as long as you would like. Thirdly, provide a space for respondents to write their rationales for their choices on which tool is more culturally relevant. Fourthly, the use of the electronic shorter versions of the tools, such as sending the respondents a web site link that has the surveys, so respondents can go online and answer the survey questions at their convenience. This method is cost effective for both researchers and respondents. This will save time and resources for the researcher in photocopying and binding, sending and receiving the questionnaires. Furthermore, it will save time and effort in entering the data electronically. Respondents will not be spending time in mailing back the survey packages.

Dialysis respondents were asked to rate how good these tools were capturing the quality of their life as shown in Table 5.12, the majority (66\% for SF-36 and 50\% for the QOL Index tool and $20 \%$ rated SF-36 as excellent compared with $24 \%$ for the QOL Index. These findings rated them as good indicate that those tools were just acceptable or average but not excellent or highly recommended. Another explanation for this finding is most of the dialysis respondents' responses were possibly based on the motivation to be a "good respondent". Hence the responses may be subject to editing rather than reflecting the respondent's true assessment (Warnecke et al., 1996).

\subsection{The usage of the QOL tools in clinical settings}

Assessing QOL as part of screening dialysis patients is encouraged among healthcare professionals (Huang et al., 2006). It is important for nurses and other healthcare professionals to include assessment of patients' personal experience when assessing effectiveness of treatment outcomes. The SF-36 and QOL Index tools were designed to be used in research only, not in the clinical settings. They are impractical to be used in the clinical settings especially when patients come for doctor visits or nurse clinics. They are too long to complete and difficult to score during clinic visits. Patients may tire of filling 
forms especially if they feel it is going to replace open communication with nurses and doctors. This could be considered as a barrier to their use in clinical settings (Higginson \& Carr, 2001). QOL tools used in clinical settings should be quick and easy for patients to complete and easy for healthcare professionals to score (Lubeck, 2002). Pincus and Wolfe (2000) proposed that for a tool to be feasible, it must be completed within 10 minutes and scored within 30 minutes. Within a clinic visit, 30 minutes to score a measurement tool would be too long and impractical; using an electronic scoring system should be developed as it would be more convenient to the nursing staff and the nephrologists. In a busy clinic setting, taking time to complete and score the tool needs to result in useful clinical information that may either guide the treatment plan or monitor the effectiveness of the treatment interventions (Higginson \& Carr). Nurses should think of creative solutions in shortening the surveys to a single page that can easily analysed is useful for monitoring health in general and specific populations. Surveys with fewer questionnaire items are easy to score and more practical to use (Agrawal, Garimella, Roshan, \& Ghosh, 2009).

The use of QOL measures within the clinic visit can provide improved assessment, recognition of the impact of illness on the individual, improved communication, comparison between patients and improved documentation (Groessl, Ganiats, \& Sarkin, 2006). It can also ensure that the focus of intervention is on the individual and not just on the disease (Higginson \& Carr). This could facilitate the implementation of the holistic nature of nursing care. Clinicians and researchers should take care in finalysing their research questions related to health-reported outcomes and in selecting which instrument to use (Huang et al., 2006). The tool should serve the purpose of the research and help in answering the research questions.

The tools need to be practical to be used in the clinical setting. The work that has been done on the translations and adaptations of the SF-12 ${ }^{\circledR}$ and the SF-6D is promising. However, the choice between the SF- $12^{\circledR}$ and the SF- $36^{\circledast}$ is a pragmatic choice about the amount of information needed. The SF- $12^{\circledR}$ and SF-6D are shorter versions of the SF-36 and have been used in several studies (Brazier \& Roberts, 2004; Brazier, Tsuchiya, Roberts, \& Busschbach, 2004; O'Brien, Spath, Blackhouse, Severens, \& Brazier, 2003). The computerised versions of the SF- $12^{\circledR}$ and SF-6D can be done easily and presented to the doctors for medical evaluation during clinic visits in conjunction with the vital signs and 
any other assessment. Numerous researchers and healthcare delivery organisations have adopted the SF $-12^{\circledR}$, including the National Commission on Quality Assurance (NCQA) which uses the SF $-12^{\circledR}$ for its Annual Member Healthcare Survey (Quality Metric, 2009). The translation of this tool into different languages is underway (Quality Metric). However, it might take a few years to have these tools translated and validated as well as to have well established reliability tests. Selecting the most appropriate tool depends on the goals of the users, the resources available, and how the results or data will be used (Amarantos et al., 2001). For any new researches in UAE, all future computerised tools should be culturally relevant to the population of UAE.

While the current work is mainly on developing computerised shorter versions of the SF-36 such as SF $-12^{\circledR}$ and the SF $-36^{\circledR}$ which are general tools that are promising, these tools may not capture the QOL-related issues to specific diseases such as cancer and kidney failure. There is a need to explore more options of developing shorter and computerised versions of some of the disease-specific tools. The shorter versions should be tested for reliability and validity and also for cultural relevancy. Nurses need to know how to assess the QOL and be familiar with the newly developed computerised versions of the QOL tools as well as be able to assess those tools for cultural relevancy. Nursing has led the way with cultural safety and providing cultural safe practice, now they have the opportunity to lead the way with developing systems that ensure the cultural relevancy of the QOL tools. Therefore, there is a need for more research on the validation process for the cultural relevancy of the tools.

\subsection{Respondents views on QOL}

The open-ended questions were asked to find out from the respondents what they feel and think is important in relation to their QOL and what contributes to it. The comparison of qualitative data between the two samples was outlined in Table 5.13. The themes identified by both samples are interrelated. In the first question respondents were asked to state what they believed other people valued most in life. This could be different from their views or beliefs about what they valued personally in life. Money was the most valued of the QOL in both samples but was more obvious in the dialysis sample. The value of money for dialysis respondents could be different from the respondents from the community sample because a considerable number of the dialysis respondents were not employed 
and most likely had minimal income. Also, non-national dialysis patients need money to have kidney transplants, while others need it for living and to meet family demands. High income is linked to good health and happy life (Belbeisi, Zindah, Walke, Jarrar, \& Mokdad, 2009). In contrast, poverty correlates with poor health and poor QOL (Giacaman, et al., 2009). Poverty has been shown to impact negatively on their QOL (Giacaman et al.). Income was not assessed in this research; this could be considered as one of the limitations of this research.

In the second question respondents were asked to state what they personally valued most in life. The majority of both samples also valued health. More than two thirds of the community sample valued health most in life compared with $60 \%$ of the dialysis sample. This could reflect that the majority of the community sample were well educated and more aware of the importance of being healthy and adopting a healthy lifestyle. Having a long term condition would mean a lot of life disruptions. Studies on patients with ESRD show that dialysis patients suffer from disease-specific symptoms (Buargub et al., 2006), reduced physical working capacity (Sterky \& Stegmayr, 2005), inability to work full-time (Neri et al., 2005) and difficulties in managing family responsibilities and social living (Mok \& Tam, 2001). Furthermore, a dialysis schedule can significantly obstruct both professional and personal lifestyle (Kimmel, 1995). Kidney failure and dialysis impact negatively on QOL, resulting in depression, anxiety, interpersonal stress, and marital conflict (Lew \& Piraino, 2005).

Family provides a sense of social security and general well-being (Gencoz \& Astan, 2006). This support the finding that more than two thirds of the community sample personally valued family in life, compared with half of the dialysis sample. Respecting older people and looking after sick members are the core values of the family in Arab countries as outlined in the conceptual framework. The number of respondents from the community sample who believed that family was valued most in life in the UAE population was almost double compared with the dialysis sample. This contradicts the expectation in the conceptual framework, that family plays a major role in the life of dialysis patients. The potentially damaging impact of ESRD on a patient's psychosocial function, and the marital strain experienced by patients on dialysis is well documented (Shidler et al., 1998). Almost similar percentages in dialysis and community samples (30.7 vs. 28.5 respectively) valued 
religion most in life. Religion also plays an important part in people's lives from Arab countries. Muslims believe that by following the Islamic religion guidelines, people will have a happy life while living and after their death. Muslims believe that there is another life after the Day of Judgment, and what decides how they are going to live in the afterlife is their current life and the degree to which they follow the religion's instructions. As outlined in the conceptual framework, belonging to a religion and the degree of religiosity will impact on the overall satisfaction with life as well as a higher degree of psychological adaptation and acceptance of illness. Religion provides a sense of spiritual and psychological security (Patel et al., 2002; Spinale et al., 2008).

In the third question respondents were asked to state what things are important to them that might improve and maintain their QOL? A quarter of the community sample believed that having a good job would have a major impact on improving and maintaining their QOL compared with an eighth of the dialysis sample. Loss of work is an important issue for dialysis patients and other people. This could be explained by the fact that some dialysis patients not coping with full-time employment due to their physical health and the time needed to do dialysis. On average, dialysis patients spend 15 hours a week on dialysis which is equivalent to part-time employment. Some patients stopped working or moved on to work part-time, which in the long term will have an impact on their income, consequently compromising their QOL and possibly changing their role in the family. Van Manen et al. (2001), and the members of Netherlands Cooperative Study on adequacy of dialysis, have found that within one year, the proportion of hemodialysis patients employed were decreased from $31 \%$ to $25 \%$ of hemodialysis patients.

New themes have been identified by dialysis respondents when they were asked about what they personally valued most in life such as kidney transplant, finding alternative to dialysis, having a social life and living with illness. However, having a kidney transplant as well as a cure from the illness were more obvious themes when asked what things were most important to them in maintaining or improving their QOL. Those themes reflected that, dialysis patients' hoped to have a cure from this chronic debilitating illness which affects not only their physical health but also their mental and psychological health and well being. Other dialysis patients hoped to have a kidney transplant. Having a kidney transplant in the Middle East and in the UAE is particularly difficult. Patients have to find a 
relative who is willing to donate a kidney to them. The availability of a relative who is willing to donate is also a challenge for non National dialysis patients.

\subsubsection{Ranking of the identified themes}

The ranking of the themes identified from the three open-ended questions that were asked at the beginning of the questionnaires were summarised in Chapter 5 Table 5.15. In general, health, money and family themes had the top priority. Each sample prioritised themes differently depending on their personal values and needs. The dialysis sample had poor health and income, so they ranked health and money as a top priority. In contrast, the community sample ranked job as a third priority when asked to list the things that were most important to them in maintaining or improving their QOL. Health and family was ranked as the first priority for the community sample, while in the dialysis sample health was ranked first and family was ranked second. Religion ranked differently in both samples in each of the three questions. However, these themes are considered universal for both respondents. As outlined in the conceptual framework, religion had a major influence on the QOL of the UAE dialysis patients and the general population. Safety, relationship, job, housing and education themes had different ranking in the three questions. New themes that emerged specifically from the dialysis sample had the lowest ranking scores such as kidney transplant, cure from illness, living with illness and alternative to dialysis.

\subsubsection{Themes identified and the level of representation in the QOL tools}

The themes that emerged from the analysis of the open-ended question and the level of representation in the QOL tools were summarised in Table 5.16. It has been shown that the QOL tools did not capture all of the themes identified in this study. This result is similar to a study done by Maor et al. (2001) in which they investigated the correlation between descriptive measures of health-related QOL using three different QOL tools (Patients Utilities (Time Trade-Off), Global Rating of HRQL and SF-36). Maor et al. confirmed that a qualitative difference exists between the Time Trade-Off tool and other descriptive QOL tools used in this study. Therefore, in this study, the use of two QOL tools and incorporating the identified themes from the qualitative data in this study helped in capturing more dimensions of the QOL from the selected samples. Furthermore, QOL Index tool with its disease specific and generic versions captured wide range dimensions of QOL compared with SF-36. SF-36 measures health related QOL issues only. In 
contrast, QOL Index was more comprehensive in nature that captured health related issues as well as other dimensions of QOL such as the impact of religion and social relationship on QOL.

\subsection{Conclusion}

The return rate in this study is high for both samples compared with many other studies. Neither sample was representative of the populations they were drawn from. The majority of both samples were married. This study explored the QOL of dialysis patients and a community sample from different age groups, major religions, different ethnicities, several living arrangements, employment, and educational levels. It has been shown that some of the questions were culturally inappropriate to some respondents. These questions need modification to match the culture, religion and tradition of the country. Researchers need to assess the QOL tools for cultural relevancy before using them with people from different cultures. But, in general both the QOL tools were culturally relevant and acceptable for use by the majority of the two samples. The number of missing data was greater when the QOL Index was used. Very few respondents from both samples considered that neither tools were culturally relevant. It was also highlighted that there is a strong relationship between the missing data and the cultural relevancy. Questions that had a higher rate of missing data were examined for their cultural applicability and relevancy.

Despite the finding that both tools were considered culturally relevant by the majority of both samples, few respondents considered both tools culturally irrelevant. The level of missing data in QOL Index tool could be less and the response rate could be higher if all the questions in the QOL Index were culturally relevant. The general results of QOL tools are valid and these results will be discussed in the next chapter. The QOL tools developed in other cultures should not only be tested for validity and reliability but also for cultural relevancy before using them with people from other ethnic or cultural backgrounds. The SF-36 and QOL Index tools were designed to be used in research only, not on the clinical settings. They are too long to complete and difficult to score during the clinic visits. The tools need to be practical to be used in the clinical setting. In addition to the work undertaken on the general tools, there is a need to explore more options of developing shorter and computerised versions of some of the disease-specific tools. The shorter versions should be tested not only for reliability and validity but also for cultural relevancy. 
The next two chapters will report and discuss the findings from the QOL tools for both samples. 


\section{Chapter 7 Findings related to Quality of life}

This is the second chapter that reports the findings from this study. Section one presents the findings from the total scores of SF-36 and QOL Index tools and their subscales scores for both samples and finding of blood results of the dialysis sample. Section two and three presents the findings from the comparisons of the independent variables and the total scores of both tools in both samples. Section four presents the comparison of the significant values of the two tools. Section five presents the results of the multiple regression analyses for the dialysis and the community samples using both tools to determine what factors influenced the QOL scores. The last section reports the findings of the MANOVA of both samples.

\subsection{Findings from QOL tools and their subscales analyses}

This phase of the statistical analysis aimed to explore the statistical differences in the subscale scores of both tools. There were 150 questionnaires eligible for analysis in the dialysis sample and were 264 questionnaires eligible for analysis in the community sample. As the data were normally distributed, an independent $t$ test was used to establish the differences between the scores of the subscales of both samples. Table 7.1 presents the comparison between the subscales and total scores of SF-36 results of both samples.

Table 7.1 Comparison between the SF-36 subscales and the total scores of both samples

\begin{tabular}{|l|r|r|c|}
\hline SF-36 Scores (0-100) & $\begin{array}{c}\text { Dialysis } \\
(\mathbf{n = 1 5 0 )} \mathbf{M} \pm \mathbf{S D}\end{array}$ & $\begin{array}{c}\text { Community } \\
(\mathbf{n = 2 6 4 )} \mathbf{M} \mathbf{\pm S D}\end{array}$ & P Value \\
\hline SF 36 Physical Function & $54.67 \pm 27.8$ & $78.93 \pm 24.4$ & $<\mathbf{0 . 0 0 0 1}$ \\
\hline SF 36 Role-Physical & $47.00 \pm 44.7$ & $83.43 \pm 31.1$ & $<\mathbf{0 . 0 0 0 1}$ \\
\hline SF 36 Body Pain & $26.07 \pm 23.1$ & $18.07 \pm 19.8$ & $<\mathbf{0 . 0 0 0 1}$ \\
\hline SF 36 General Health & $52.53 \pm 15.5$ & $45.25 \pm 12.3$ & $<\mathbf{0 . 0 0 0 1}$ \\
\hline SF 36 Vitality & $55.91 \pm 13.7$ & $52.33 \pm 11.6$ & $\mathbf{0 . 0 0 8}$ \\
\hline SF 36 Social Functioning & $44.58 \pm 18.2$ & $46.88 \pm 13.6$ & 0.181 \\
\hline SF 36 Role Emotional & $55.33 \pm 46.3$ & $76.03 \pm 77.1$ & $<\mathbf{0 . 0 0 0 1}$ \\
\hline SF 36 Mental Health & $58.75 \pm 12.6$ & $59.14 \pm 10.8$ & 0.747 \\
\hline SF 36 Total Score & $58.92 \pm 19.2$ & $75.02 \pm 16.3$ & $<\mathbf{0 . 0 0 0 1}$ \\
\hline
\end{tabular}

$t$ test

The Physical Health Component (PHC) of the SF-36 includes physical function, rolephysical, body pain and general health subscales. The Mental Health Component (MHC) 
includes vitality, social functioning, role emotional and mental health subscales. In the dialysis sample, the mean total score of the $\mathrm{PHC}$ was 45.1 and the $\mathrm{MHC}$ was 53.6. On the other hand the mean total score of the PHC for the community sample was 56.4 and the MHC was 58.6. The highest mean scores in the dialysis sample with regards to the SF-36 were in the mental health subscale (58.8) and the lowest scores were in body pain subscale (26.1). In the community sample, the highest mean scores were in the role physical subscale (83.4) and the lowest scores were in the body pain subscale (18.0).

It is apparent from the above table that the community sample had statistically significant better scores in all subscales including the total scores of the SF-36, except social functioning and mental health subscales. Dialysis respondents had statistically significant better scores on the body pain and general health subscales. Furthermore, the community sample on average had almost double the scores on the role-physical subscale compared with the dialysis sample. The community sample rated on average 8.1 points lower than the dialysis sample on the body pain subscale. Moreover, the community sample scored on average 7.2 points lower than the dialysis sample on the general health subscale. Likewise, the community sample rated on average 3.6 points lower than the dialysis sample on the vitality subscale. Additionally, the community sample rated on average 21.7 points higher than the dialysis sample on the role emotional subscale. In general, the total score for the SF-36 was in favour of the community sample by on average16.1 points on a scale from 0-100. Table 7.2 presents the comparison between the subscales and total scores of QOL Index results of both samples.

Table 7.2 Comparison between subscales and total scores of QOL Index of both samples

\begin{tabular}{|l|r|r|r|r|r|}
\hline $\begin{array}{c}\text { Quality of Life Index subscales } \\
\text { scores (0-30) }\end{array}$ & $\begin{array}{c}\text { Dialysis } \\
(\mathbf{n = 1 5 0 )} \mathbf{M} \mathbf{S D}\end{array}$ & $\begin{array}{c}\text { \% or out } \\
\text { of 100 }\end{array}$ & $\begin{array}{c}\text { Community } \\
(\mathbf{n = 2 6 7 )} \mathbf{M} \pm \mathbf{S D}\end{array}$ & $\begin{array}{c}\text { \% out } \\
\text { of 100 }\end{array}$ & $\begin{array}{c}\mathbf{p} \\
\text { Value }\end{array}$ \\
\hline Health and Functioning & $21.59 \pm 5.9$ & 71.9 & $23.30 \pm 4.8$ & 77.6 & $\mathbf{0 . 0 0 1}$ \\
\hline Social and Economic & $23.13 \pm 5.4$ & 77.0 & $22.32 \pm 4.9$ & 74.4 & 0.118 \\
\hline Psychological/Spiritual & $24.26 \pm 6.2$ & 80.8 & $24.32 \pm 5.1$ & 81.1 & 0.925 \\
\hline Family & $26.33 \pm 4.5$ & 87.7 & $24.82 \pm 5.6$ & 82.7 & $\mathbf{0 . 0 0 5}$ \\
\hline Quality of Life Index Total Score & $23.18 \pm 5.1$ & 77.2 & $23.57 \pm 4.5$ & 78.6 & 0.421 \\
\hline
\end{tabular}

$t$ test 
The QOL Index scores ranged from 0 to 30 , with 0 being the lowest score and 30 being the highest score. For an accurate and easy comparison of both tools, the QOL Index scores were transformed to be from $0-100$. On this scale, 0 is the lowest score, while 100 is the highest score. Findings on the QOL Index for the dialysis sample were all well above the midpoint of the scale and subscale. The highest score for the dialysis sample was in the family subscale followed by the psychological/spiritual subscale, and the lowest in the health and functioning subscale. Mean findings from the QOL Index for the community sample were also all well above the midpoint of the scale and subscales. The highest mean score for the community sample was also in the family subscale followed by the psychological/spiritual subscale. The lowest mean scores were in the social and economic subscale. The above table shows that the community sample had statistically higher mean scores on two of the four subscales. The dialysis sample scored approximately six points less than the community sample in the health and functioning subscale. On the other hand, the dialysis sample had a statistically significant better mean score on the family subscale compared with the community sample.

Given that none of the subscale components of the two tools are directly comparable, a comparison of scores of the two instruments can only be made on the total scores. This comparison indicates that dialysis respondents' overall mean QOL is rated higher when self-assessed using the QOL Index (77.2 vs 58.92) than the SF-36. In contrast, the comparison indicated that the overall mean QOL of the community sample was almost similar when self-assessed using the QOL Index and the SF-36 (78.60, 75.02 respectively).

\subsubsection{Blood results of the dialysis sample}

The majority of dialysis patients at SKMC receive four hours of Hi Flux dialysis three times a week. The average time respondents had been on dialysis was $4.4 \pm 54.2$ year range (0.08-24.9) years. There were 20 patients out of the total sample of the dialysis patients who did not have their blood test done on this month, so the blood results presented in Table 7.3 represented only 130 patients. 
Table 7.3 Blood results of the dialysis sample

\begin{tabular}{|c|c|c|c|}
\hline Variable & $\begin{array}{l}\text { Mean } \pm S D \\
(n=130)\end{array}$ & Range & Therapeutic values $^{*}$ \\
\hline HB & $\begin{array}{l}117.2 \pm 18.0 \\
118.6 \pm 15.6\end{array}$ & $69-157$ & $\begin{array}{r}\text { Male: } 113-118 \mathrm{~g} / \mathrm{L} \\
\text { Female: } 112-116 \mathrm{~g} / \mathrm{L}\end{array}$ \\
\hline Albumin & $33.4 \pm 4.9$ & $18-55$ & $35-48 \mathrm{~g} / \mathrm{L}$ \\
\hline Pre-dialysis urea (g/L) & $22.2 \pm 7.2$ & $5.8-58.8$ & $1.2-6.4 \mathrm{mmol} / \mathrm{L}$ \\
\hline Pre-dialysis creatinine $(\mathrm{g} / \mathrm{L})$ & $967.1 \pm 268.9$ & 83-1982 & $62-115 \mu \mathrm{mol} / \mathrm{L}$ \\
\hline Urea reduction ratio & $75.3 \pm 6.9$ & $56.2-91$ & Above 65 \\
\hline
\end{tabular}

The average haemoglobin level appeared to be within the normal range for males and above the therapeutic level for females. This therapeutic level is for people without kidney failure. More than half of the dialysis male sample had a haemoglobin level above the range and around one third were below the range, leaving $11.5 \%$ within the therapeutic level. One quarter of the female dialysis respondents had a haemoglobin level below the therapeutic range and $61.2 \%$ had haemoglobin level above the range leaving 3 respondents only within the normal range. More than half of the dialysis sample had a serum albumin level below the therapeutic range with only 2 patients above the range. Nearly all patients had a urea and creatinine levels above the therapeutic range except one respondent who had a therapeutic range of urea. There were $7.9 \%$ who had urea reduction ratio below $65 \%$. Moreover, there were 20 dialysis patients who had not blood investigation during the month of data collection.

\subsection{The relationship between the SF-36 total scores and the independent variables in both samples}

This section presents the findings from the comparisons of the independent variables and SF-36 total scores in both samples. This phase of the analysis involved a series of statistical tests to establish what socio-demographics and life factors correlated with or had an impact on the SF-36 and QOL Index total scores. Pearson's correlations were used for continuous variables, Spearman's correlation for ordinal data and $t$ test or one way ANOVA for nominal variables. Table 7.4 summarises the findings from the comparison between the demographic variables and SF-36 total. 
Table 7.4 Comparison of the findings between the demographic variables and SF-36 total scores in both samples

\begin{tabular}{|c|c|c|c|c|c|}
\hline Variable & Values & $\begin{array}{c}\text { Dialysis Mean } \\
\text { (SD) } \\
\text { Max }=100\end{array}$ & $p$ value & $\begin{array}{l}\text { Community } \\
\text { Mean (SD) } \\
\text { Max }=100\end{array}$ & $p$ value \\
\hline Gender* & $\begin{array}{l}\text { Male } \\
\text { Female }\end{array}$ & $\begin{array}{l}60.7 \pm 19.6 \\
54.7 \pm 18.0\end{array}$ & 0.084 & $\begin{array}{l}77.4 \pm 15.6 \\
71.9 \pm 16.7\end{array}$ & 0.006 \\
\hline Ethnicity** & $\begin{array}{l}\text { UAE National } \\
\text { Arab National } \\
\text { South Asian } \\
\text { Other }\end{array}$ & $\begin{array}{l}55.6 \pm 18.7 \\
57.2 \pm 19.4 \\
64.6 \pm 19.6 \\
61.4 \pm 17.3\end{array}$ & 0.168 & $\begin{array}{l}72.9 \pm 18.3 \\
72.1 \pm 16.9 \\
81.9 \pm 12.8 \\
75.9 \pm 15.4\end{array}$ & 0.001 \\
\hline Marital status ${ }^{\star \star}$ & $\begin{array}{l}\text { Single } \\
\text { Married } \\
\text { Divorced or widowed }\end{array}$ & $\begin{array}{l}61.3 \pm 14.2 \\
59.6 \pm 19.6 \\
54.0 \pm 20.3\end{array}$ & 0.413 & $\begin{array}{l}72.4 \pm 14.6 \\
75.9 \pm 16.4 \\
72.8 \pm 20.7\end{array}$ & 0.336 \\
\hline Religion** & $\begin{array}{l}\text { Muslim } \\
\text { Christian } \\
\text { Others } \\
\end{array}$ & $\begin{array}{l}58.3 \pm 19.5 \\
64.6 \pm 17.5 \\
57.2 \pm 19.1 \\
\end{array}$ & 0.457 & $\begin{array}{l}73.4 \pm 17.4 \\
78.5 \pm 13.4 \\
77.8 \pm 13.3 \\
\end{array}$ & 0.064 \\
\hline $\begin{array}{l}\text { Living } \\
\text { arrangements** }\end{array}$ & $\begin{array}{l}\text { Lives alone } \\
\text { Lives with family } \\
\text { Other }\end{array}$ & $\begin{array}{l}57.9 \pm 18.1 \\
60.2 \pm 25.3 \\
61.3 \pm 20.4\end{array}$ & 0.644 & $\begin{array}{l}78.0 \pm 13.6 \\
73.5 \pm 16.6 \\
80.6 \pm 15.6\end{array}$ & 0.031 \\
\hline Employment $^{\star \star}$ & $\begin{array}{l}\text { Full time employment } \\
\text { Housekeeper, student, } \\
\text { part time employed } \\
\text { Retired and disabled } \\
\text { Unemployed } \\
\end{array}$ & $\begin{array}{l}63.7 \pm 20.2 \\
57.1 \pm 18.5 \\
48.7 \pm 17.7 \\
60.4 \pm 16.2 \\
\end{array}$ & 0.009 & $\begin{array}{r}77.6 \pm 14.5 \\
63.3 \pm 18.7 \\
56.6 \pm 20.9 \\
73.9 \pm 17.7 \\
\end{array}$ & $<0.0001$ \\
\hline $\begin{array}{l}\text { Level of } \\
\text { education** }\end{array}$ & $\begin{array}{l}\text { Did not attend school } \\
\text { Primary school } \\
\text { Secondary school } \\
\text { Tertiary school }\end{array}$ & $\begin{array}{l}50.8 \pm 19.7 \\
61.6 \pm 21.3 \\
61.6 \pm 17.7 \\
60.0 \pm 16.5\end{array}$ & 0.057 & $\begin{array}{l}66.9 \pm 28.2 \\
72.0 \pm 16.9 \\
75.5 \pm 15.6 \\
75.3 \pm 16.0\end{array}$ & 0.566 \\
\hline $\begin{array}{l}\text { Long term } \\
\text { conditions }\end{array}$ & $\begin{array}{l}\text { Yes } \\
\text { No }\end{array}$ & $\begin{array}{l}51.5 \pm 17.9 \\
67.5 \pm 17.1\end{array}$ & $<0.0001$ & $\begin{array}{l}67.7 \pm 20.7 \\
76.4 \pm 15.2\end{array}$ & 0.002 \\
\hline Life event ${ }^{*}$ & $\begin{array}{l}\text { Yes } \\
\text { No }\end{array}$ & $\begin{array}{l}54.8 \pm 18.0 \\
60.7 \pm 19.6\end{array}$ & 0.083 & $\begin{array}{l}69.2 \pm 17.1 \\
76.2 \pm 15.9\end{array}$ & 0.002 \\
\hline $\begin{array}{l}\text { Cause of } \\
\text { kidney failure* }\end{array}$ & $\begin{array}{l}\text { Yes } \\
\text { No }\end{array}$ & $\begin{array}{l}61.1 \pm 20.2 \\
54.3 \pm 16.2 \\
\end{array}$ & 0.043 & $\mathrm{~N} / \mathrm{A}$ & $\mathrm{N} / \mathrm{A}$ \\
\hline
\end{tabular}

${ }^{*} t$ test $\quad{ }^{* \star}$ one-way ANOVA test

The $t$ test and ANOVA compare averages, so throughout the study the differences in reference to these tests refer to the differences on average of the total group scores, not individuals. The $t$-test comparison of the total SF-36 scores with the nominal demographic variables found that dialysis respondents with another long term condition and community respondents with long term condition had a statistically significant lower QOL scores using SF-36 ( $p=<0.0001,0.002$ respectively). Those in the dialysis sample who had full-time employment had significantly higher mean SF-36 total scores. Male gender, South east Asian ethnicity, other living arrangements and no major life event variables had a 
statistically significantly higher mean SF-36 total scores in the community sample ( $p=$ $0.006,0.001,0.031$ and 0.002 respectively). Respondents from the community sample who were identified as South East Asian were an average 9 point higher than UAE national respondents and 9.8 points higher than the Arab national sample and 6 points higher than respondents from other nationalities. In the dialysis sample, male gender and not having any major life events both trends toward a statistically significant influence of the total scores of the SF-36 ( $p=0.084,0.057$ respectively).

The one-way ANOVA test comparison on the SF-36 total scores with the categorical demographic variables found that employment variable had a statistically significant impact on the total scores of SF-36 for both samples ( $p=0.009,<0.0001$ respectively). In the dialysis sample, respondents who had a full time employment had on average 6.6 points higher than them (house keepers, students, and part time employed groups). Dialysis patients who were disabled or retired had an average score of 15 points lower than those who were employed in full time jobs. In contrast, in the community sample respondents who had a full time employment had on average 14.3 points higher than those who were either student or part time or keeping house and they had 21 points higher than those who were disabled and retired and 3.7 points higher than respondent who were not employed. Furthermore, those who knew the cause of their kidney failure had a statistically significant higher QOL scores ( $p=0.043$ ) than those who did not know the cause of their kidney failure. In the community sample, respondents with Christian religion have a trends toward having a statistically significant influence of the total scores of the SF-36 compared with Muslim respondents $(p=0.064)$.

Table 7.5 summarises the correlations with the SF-36 total scores and the continuous demographic variables. The table shows that the mean age for the dialysis sample is higher than the community sample by around 8.5 years. The age variable has a statistically significant positive correlation with the total scores of the SF-36 ( $p=0.045)$ in the community sample. The length of time community respondents lived in and travelled out of the UAE had trends toward having a statistically significant negative correlation with the total scores of SF-36 ( $p=0.059,0.073$ respectively). 
Table 7.5 Correlation between demographic data and SF-36 total scores

\begin{tabular}{|l|r|r|r|r|r|r|}
\hline Variables & $\begin{array}{c}\text { Dialysis } \\
\text { Mean } \\
\text { (range) }\end{array}$ & $\begin{array}{c}\text { Pearson } \\
\text { coefficie } \\
\text { nt }(\mathbf{r})\end{array}$ & $\begin{array}{c}\mathbf{p} \\
\text { values }\end{array}$ & $\begin{array}{c}\text { Community } \\
\text { Mean } \\
\text { (range) }\end{array}$ & $\begin{array}{c}\text { Pearson } \\
\text { coefficient } \\
(\mathbf{r})\end{array}$ & $\begin{array}{c}\mathbf{p} \\
\text { value }\end{array}$ \\
\hline Age in years & $\begin{array}{r}49.05 \\
(19-86)\end{array}$ & -0.133 & 0.105 & $\begin{array}{r}40.49 \\
(18-69)\end{array}$ & 0.105 & $\mathbf{0 . 0 4 5}$ \\
\hline $\begin{array}{l}\text { Length of time living } \\
\text { in UAE in years }\end{array}$ & $\begin{array}{r}26.81 \\
(0.3-86.0)\end{array}$ & -0.091 & 0.269 & $\begin{array}{r}15.70 \\
(0.16-6)\end{array}$ & -0.097 & 0.059 \\
\hline $\begin{array}{l}\text { Last time travelled } \\
\text { out of UAE in years }\end{array}$ & $\begin{array}{r}3.86 \\
(0-40)\end{array}$ & -0.077 & 0.349 & $\begin{array}{r}1.45 \\
(0-37.4)\end{array}$ & -0.080 & 0.073 \\
\hline
\end{tabular}

The findings of the Pearson correlations were done to establish the correlation between the SF-36 total scores and the clinical variables. The collected laboratory values were from the dialysis sample only. The findings are presented in Table 7.6.

Table 7.6.Clinical variables correlations with SF-36 total scores for the dialysis sample

\begin{tabular}{|l|r|r|r|}
\hline Variables & Mean $(\mathbf{n = 1 3 0})(\mathbf{r a n g e})$ & Pearson coefficient $(\mathbf{r})$ & P values \\
\hline HB & $117.59(\mathrm{~g} / \mathrm{dL})$ & 0.102 & 0.246 \\
\hline Albumin & $33.35(\mathrm{~g} / \mathrm{dL})$ & 0.108 & 0.221 \\
\hline Urea pre dialysis & $22.22 \mathrm{mmol} / \mathrm{L}$ & 0.096 & 0.276 \\
\hline Creatinine pre dialysis & $960.23 \mu \mathrm{mol} / \mathrm{L}$ & 0.274 & $\mathbf{0 . 0 0 2}$ \\
\hline Urea Reduction Ratio (URR) & $75.29 \%$ & -0.116 & 0.195 \\
\hline $\begin{array}{l}\text { Length of time on dialysis in } \\
\text { years }\end{array}$ & 4.4 & 0.027 & 0.745 \\
\hline
\end{tabular}

The above table showed that pre-dialysis serum creatinine level had a statistically significant correlation with the SF-36 total scores. Other clinical variables did not have any statistically significant correlation with the SF-36 total scores.

\subsection{The relationship between the QOL Index total scores and the independent variables in both samples}

This section presents the findings from the comparison of the demographic variables and the QOL Index total scores in both samples (Table 7.7). 
Table 7.7 Comparison of the findings between the demographic variables and QOL Index total scores for both samples

\begin{tabular}{|c|c|c|c|c|c|}
\hline Variable & Values & $\begin{array}{c}\text { Dialysis Mean } \\
\text { (SD) } \\
\text { Max }=30\end{array}$ & $p$ value & $\begin{array}{c}\text { Community } \\
\text { Mean (SD) } \\
\text { Max }=100\end{array}$ & $p$ value \\
\hline Gender* & $\begin{array}{l}\text { Female } \\
\text { Male }\end{array}$ & $\begin{array}{l}22.9 \pm 5.2 \\
23.3 \pm 4.7\end{array}$ & 0.671 & $\begin{array}{l}23.3 \pm 4.6 \\
23.8 \pm 4.4\end{array}$ & 0.360 \\
\hline Ethnicity ** & $\begin{array}{l}\text { UAE National } \\
\text { Arab National } \\
\text { South Asian } \\
\text { Other }\end{array}$ & $\begin{array}{l}25.2 \pm 4.1 \\
22.2 \pm 5.4 \\
22.6 \pm 5.2 \\
22.5 \pm 5.1\end{array}$ & 0.023 & $\begin{array}{l}23.6 \pm 5.3 \\
22.6 \pm 4.5 \\
26.0 \pm 3.2 \\
22.9 \pm 3.9\end{array}$ & $<0.0001$ \\
\hline Marital status ${ }^{\star *}$ & $\begin{array}{l}\text { Single } \\
\text { Married } \\
\text { Divorced or widowed }\end{array}$ & $\begin{array}{l}23.2 \pm 4.0 \\
23.4 \pm 5.1 \\
22.3 \pm 5.6\end{array}$ & 0.690 & $\begin{array}{l}22.3 \pm 4.2 \\
24.0 \pm 4.4 \\
22.0 \pm 6.6\end{array}$ & 0.020 \\
\hline Religion ** & $\begin{array}{l}\text { Muslim } \\
\text { Christians } \\
\text { Others }\end{array}$ & $\begin{array}{l}23.4 \pm 5.0 \\
22.6 \pm 5.4 \\
21.6 \pm 5.4\end{array}$ & 0.472 & $\begin{array}{l}23.1 \pm 4.8 \\
24.8 \pm 3.5 \\
24.0 \pm 4.1\end{array}$ & 0.020 \\
\hline $\begin{array}{l}\text { Living } \\
\text { arrangements** }\end{array}$ & $\begin{array}{l}\text { Lives with family } \\
\text { Lives alone } \\
\text { Other }\end{array}$ & $\begin{array}{l}22.8 \pm 7.5 \\
23.9 \pm 4.6 \\
21.4 \pm 5.0\end{array}$ & 0.038 & $\begin{array}{l}23.6 \pm 4.5 \\
22.5 \pm 4.5 \\
24.3 \pm 4.2 \\
\end{array}$ & 0.230 \\
\hline Employment $^{\star *}$ & $\begin{array}{l}\text { Full time employment } \\
\text { Housekeeper, student, } \\
\text { part time employed } \\
\text { Retired and disabled } \\
\text { Unemployed }\end{array}$ & $\begin{array}{l}23.1 \pm 5.3 \\
23.2 \pm 4.9 \\
24.1 \pm 5.6 \\
22.5 \pm 4.6\end{array}$ & 0.705 & $\begin{array}{l}24.1 \pm 4.2 \\
21.3 \pm 4.8 \\
19.4 \pm 7.5 \\
22.8 \pm 4.5\end{array}$ & $<0.0001$ \\
\hline $\begin{array}{l}\text { Level of } \\
\text { education** }\end{array}$ & $\begin{array}{l}\text { Did not attend school } \\
\text { Primary school } \\
\text { Secondary school } \\
\text { Tertiary school }\end{array}$ & $\begin{array}{l}23.0 \pm 4.0 \\
23.3 \pm 5.6 \\
23.5 \pm 5.3 \\
22.8 \pm 5.2\end{array}$ & 0.936 & $\begin{array}{l}22.1 \pm 6.2 \\
22.6 \pm 5.5 \\
23.9 \pm 4.6 \\
23.6 \pm 4.4\end{array}$ & 0.687 \\
\hline $\begin{array}{l}\text { Long term } \\
\text { condition* }\end{array}$ & $\begin{array}{l}\text { Yes } \\
\text { No }\end{array}$ & $\begin{array}{l}22.5 \pm 5.3 \\
24.0 \pm 4.6\end{array}$ & 0.058 & $\begin{array}{l}21.9 \pm 4.7 \\
23.9 \pm 4.4\end{array}$ & 0.011 \\
\hline Life event * & $\begin{array}{l}\text { Yes } \\
\text { No }\end{array}$ & $\begin{array}{l}22.4 \pm 5.1 \\
23.5 \pm 5.0\end{array}$ & 0.184 & $\begin{array}{l}23.2 \pm 4.1 \\
23.7 \pm 4.6\end{array}$ & 0.490 \\
\hline $\begin{array}{l}\text { Cause of } \\
\text { kidney failure* }\end{array}$ & $\begin{array}{l}\text { Yes } \\
\text { No }\end{array}$ & $\begin{array}{l}23.6 \pm 5.0 \\
22.3 \pm 5.1\end{array}$ & 0.148 & $\mathrm{~N} / \mathrm{A}$ & $\mathrm{N} / \mathrm{A}$ \\
\hline
\end{tabular}

${ }^{*} t$ test $\quad{ }^{* *}$ one-way ANOVA test

The $t$-test comparison of average QOL Index total scores with the nominal demographic variables found that long term condition variable was statistically significant in both samples ( $p=0.058,0.011$ respectively). The one-way ANOVA test comparison of the QOL Index total scores with the categorical demographic variables found that ethnicity variable had a statistically significant impact on the total scores for the dialysis and community samples ( $p=0.023,<0.0001$ respectively). The above table shows that UAE National dialysis patients had 3.0 points higher than Arab Nationals and 2.6 point higher than South East Asian and 2.7 points higher than respondents from other nationalities on average. 
Furthermore, respondents from the community sample who belong to South East Asia had 2.4 point higher than UAE national respondents and 2.4 points higher than the Arab national sample and 3.1 points higher than respondents from other nationalities on average. Living arrangement variable had a statistically significant impact on the QOL Index total scores for the dialysis sample $(p=0.038)$. Dialysis respondents who lived alone had on average better scores compared with other patients who either lived with family or have other living arrangements on average. Moreover, married respondents from the community sample had a statistically significant higher scores $(p=0.020)$. Christians had on average 1.7 points higher than Muslim respondents and 0.8 points higher than respondents from other religions. Employment variable had a statistically significant impact on the QOL Index total scores in the community sample $(p=0.001)$. Respondents from the community sample who had full time employment had on average. 2.8 points higher than those who were either student or part time or keeping house and they had 4.7 points higher than those who were disabled and retired and 1.3 points higher than respondent who were not employed. Further results are summarised in the above table.

The Pearson correlations between the QOL Index total scores and the continuous demographic variables Table 7.8 summarises the correlations with the QOL Index total scores and the continuous demographic variables.

Table 7.8 Correlation between demographic data and QOL Index total scores

\begin{tabular}{|c|c|c|c|c|c|c|}
\hline Variables & $\begin{array}{l}\text { Dialysis } \\
\text { Mean } \\
(\mathrm{n}=150) \\
\text { (range) }\end{array}$ & $\begin{array}{l}\text { Pearson } \\
\text { coefficient } \\
\quad(r)\end{array}$ & $\begin{array}{c}P \\
\text { value }\end{array}$ & $\begin{array}{c}\text { Community } \\
\text { Mean }(n=267) \\
\text { (range) }\end{array}$ & $\begin{array}{l}\text { Pearson } \\
\text { coefficient } \\
\text { (r) }\end{array}$ & $\begin{array}{l}P \\
\text { value }\end{array}$ \\
\hline Age in years & $\begin{array}{r}49.05 \\
(19-86)\end{array}$ & 0.139 & 0.089 & $40.49(18-69$ & 0.163 & 0.008 \\
\hline $\begin{array}{l}\text { Length of time } \\
\text { living in UAE in } \\
\text { years }\end{array}$ & $\begin{array}{r}26.81 \\
(0- \\
86.00) \\
\end{array}$ & 0.244 & 0.003 & $\begin{array}{r}15.70 \\
(0.16-64)\end{array}$ & -0.025 & 0.689 \\
\hline $\begin{array}{l}\text { Last time } \\
\text { travelled out of } \\
\text { UAE in years }\end{array}$ & $\begin{array}{r}3.86 \\
(0-40)\end{array}$ & $<0.0001$ & 0.998 & $1.45(0-37.4)$ & -0.080 & 0.196 \\
\hline
\end{tabular}

This table showed that in the dialysis sample, the length of time living in UAE had a statistically significant correlation with the QOL Index total scores The longer the dialysis sample lived in the UAE correlates positively with the total scores of the QOL Index $(r=$ 
$0.244, p=0.003)$. The age variable in the community sample had a statistically significant correlation with the QOL Index total scores. Increasing the age of the respondent correlated positively with the QOL Index total scores $(r=0.163, p=0.008)$. Furthermore, the age variable in the dialysis sample had trend toward being statistically significant correlation with the QOL Index total scores $(r=0.139, p=0.089)$. The rest of the variables did not have any statistically significant correlation with the total scores of the QOL Index tool.

For the dialysis sample only, Pearson correlation was done to establish the correlation between the QOL Index total scores and the collected laboratory values. The findings are presented in Table 7.9 .

Table 7.9 Lab values correlations with total scores QOL Index

\begin{tabular}{|l|r|r|r|}
\hline Lab tests & \multicolumn{1}{|l|}{ Mean } & $\begin{array}{r}\text { Pearson coefficient } \\
(\mathbf{r})\end{array}$ & \multicolumn{1}{c|}{ p-value } \\
\hline HB & $117.59(\mathrm{~g} / \mathrm{dL})$ & -0.011 & 0.899 \\
\hline Albumin & $33.35(\mathrm{~g} / \mathrm{dL})$ & 0.041 & 0.640 \\
\hline Urea pre dialysis & $22.22 \mathrm{mmol} / \mathrm{L}$ & 0.108 & 0.219 \\
\hline Creatinine pre dialysis & $960.23 \mu \mathrm{mol} / \mathrm{L}$ & 0.163 & $\mathbf{0 . 0 6 3}$ \\
\hline Urea reduction ratio (URR) & 75.29 & -0.130 & 0.146 \\
\hline $\begin{array}{l}\text { Length (range) of time on dialysis in } \\
\text { years }\end{array}$ & 4.4 & -0.127 & 0.122 \\
\hline
\end{tabular}

Pre-dialysis creatinine level has trends toward having statistically significant positive correlation with the total scores of QOL Index ( $(r=0.163, p=0.063)$. The rest of the other clinical laboratory variables did not have any statistically significant correlation with the total scores of the QOL Index tool.

\section{4 Comparison of the significant values of the two tools}

The comparison of the statistically significant values are summarised in Table 7.10. This table shows significant findings on one or both tools for one or both samples on one or two variables. Some variables had trends toward being statistically significant. In this table $p$ values that indicate a trend (less than $p=0.010$ ) are highlighted in italic. 
Table 7.10 Significant findings of the SF 36 and QOL Index for the dialysis sample

\begin{tabular}{|c|c|c|c|c|}
\hline & \multicolumn{2}{|l|}{ Dialysis } & \multicolumn{2}{|c|}{ Community } \\
\hline Variable & $\begin{array}{r}\text { SF-36 p } \\
\text { values }\end{array}$ & $\begin{array}{l}\text { QOL Index p } \\
\text { value }\end{array}$ & $\begin{array}{r}\text { SF-36 p } \\
\text { values }\end{array}$ & $\begin{array}{l}\text { QOL Index p } \\
\text { value }\end{array}$ \\
\hline Gender* & 0.084 & 0.671 & 0.006 & 0.360 \\
\hline Ethnicity** & 0.168 & 0.023 & 0.001 & $<0.0001$ \\
\hline Marital status ${ }^{\star \star}$ & 0.413 & 0.690 & 0.336 & 0.020 \\
\hline Religion ${ }^{\star *}$ & 0.457 & 0.472 & 0.064 & 0.020 \\
\hline Living arrangements** & 0.644 & 0.038 & 0.031 & 0.230 \\
\hline Employment $^{\star \star}$ & 0.009 & 0.705 & $<0.0001$ & $<0.0001$ \\
\hline Level of education ${ }^{\star *}$ & 0.057 & 0.936 & 0.566 & 0.687 \\
\hline Long term condition* & $<0.0001$ & 0.058 & 0.002 & 0.011 \\
\hline Life event $^{*}$ & 0.083 & 0.184 & 0.002 & 0.490 \\
\hline Cause of kidney failure* & 0.043 & 0.148 & $\mathrm{~N} / \mathrm{A}$ & $\mathrm{N} / \mathrm{A}$ \\
\hline Age in years ${ }^{\star \star \star}$ & 0.105 & 0.089 & 0.045 & 0.008 \\
\hline $\begin{array}{l}\text { Length of time living in UAE in } \\
\text { years }\end{array}$ & 0.269 & 0.003 & 0.059 & 0.689 \\
\hline $\begin{array}{l}\text { Last time travelled out of UAE in } \\
\text { years }\end{array}$ & 0.349 & 0.998 & 0.073 & 0.196 \\
\hline $\mathrm{HB}^{*}$ & 0.246 & 0.899 & $\mathrm{~N} / \mathrm{A}$ & $\mathrm{N} / \mathrm{A}$ \\
\hline Albumin* & 0.221 & 0.640 & $\mathrm{~N} / \mathrm{A}$ & $\mathrm{N} / \mathrm{A}$ \\
\hline Urea pre dialysis* & 0.276 & 0.219 & $\mathrm{~N} / \mathrm{A}$ & $\mathrm{N} / \mathrm{A}$ \\
\hline Creatinine pre dialysis* & 0.002 & 0.063 & $\mathrm{~N} / \mathrm{A}$ & $\mathrm{N} / \mathrm{A}$ \\
\hline Urea Reduction Ratio (URR) $^{*}$ & 0.195 & 0.146 & $\mathrm{~N} / \mathrm{A}$ & $\mathrm{N} / \mathrm{A}$ \\
\hline Length of time on dialysis in years & 0.745 & 0.122 & N/A & $\mathrm{N} / \mathrm{A}$ \\
\hline
\end{tabular}

${ }^{*}$ t test ${ }^{* *}$ one-way ANOVA test ${ }^{* * *}$ Pearson test

From the above table, it can be seen that the long term condition variable had a statistically significant negative association with the total scores of both tools for the community sample, while in the dialysis sample long term condition variable had a statistically significant negative effect on the SF-36 and trended towards statistical significance in the QOL Index. In the dialysis sample, having full time employment and knowing the cause of kidney failure and having high creatinine level had a statistically significant better scores of the SF-36. The high creatinine level has trends toward being statistically significant on the total scores of the QOL Index.

In the community sample, being male, married, having a South Asian ethnicity and being older had statistically significant association with the total scores of the QOL Index but they did not have any statistically significant effect on the total scores of the SF-36. The significant values for the community sample in both tools revealed that, four variables had statistically significant values on the total scores of both tools (long term condition, ethnicity, employment and age). This indicates that not having long term condition, having 
UAE nationality when using SF-36 or having South East Asian Nationality when using QOL Index, having full time employment and being at younger age had higher QOL scores. On the other hand, seven variables had statistically significant values on the total scores of the SF-36 (gender, ethnicity, living arrangements, employment, long term condition, life event and age variables). This indicates that being a male, having a South East Asian ethnicity, having other living arrangement, being employed full time, not having any long term condition, not having any major life events in the last 12 months and being at younger age had a statistically significant positive influence of the SF-36 total scores.

\subsection{Multiple regression findings}

To identify the factors that might have a significant influence on QOL tools for the dialysis and community samples, standard multiple regression analyses were undertaken. They were done separately using both QOL tools to examine the effect of the predictor variables on the outcome variables.

\subsubsection{Multiple regression results dialysis sample using SF-36}

The multiple regression results on the demographic variables for dialysis sample shows that a significant model has been emerged for SF-36 results ( $p=<0.0001$ ). Table 6.10 presents the regression co-efficient values of SF-36 with the demographic variables for dialysis sample. It shows that variables such as chronic health problems and cause of kidney failure had statistically significant determinants of the SF-36 total scores. This means that having another long term condition had the strongest contribution in explaining the total scores of the SF-36 and respondents who did not know the cause of their kidney failure also had a contribution in explaining the total scores of the SF-36 in dialysis respondents. Other demographic variables did not show any statistically significance. The adjusted $R^{2}$ value for this model was 0.191 , which indicates that this model accounts for $19.1 \%$ of variation in the SF-36 total scores.

The multiple regression results on categorical variable of the dialysis sample using dummy variables approach for the SF-36 presented in Table 7.11 and showed statistically insignificant model $(p=0.296)$. 
Table 7.11 Multiple regression analysis of SF-36 total scores with demographic variables for dialysis sample

\begin{tabular}{|c|c|c|c|c|c|}
\hline \multirow[t]{2}{*}{ Variables } & \multirow{2}{*}{$\begin{array}{l}\text { Std } \\
\text { Error of } \\
\beta\end{array}$} & \multirow{2}{*}{$\begin{array}{l}\text { Standard } \\
\text { Coefficient } \\
\text { ( } \beta \text { Value) }\end{array}$} & \multirow[t]{2}{*}{$p$ value } & \multicolumn{2}{|c|}{$\begin{array}{l}\text { 95\% Confidence Interval for } \\
\beta\end{array}$} \\
\hline & & & & Lower Bound & Upper Bound \\
\hline Gender & 3.183 & -0.106 & 0.163 & -10.759 & 1.824 \\
\hline Age & 0.134 & -0.097 & 0.267 & -0.414 & 0.116 \\
\hline $\begin{array}{l}\text { Chronic health } \\
\text { problems }\end{array}$ & 3.060 & 0.399 & $<0.0001$ & 9.333 & 21.431 \\
\hline Living in UAE & 0.008 & 0.108 & 0.230 & -0.006 & 0.025 \\
\hline $\begin{array}{l}\text { Cause of kidney } \\
\text { failure }\end{array}$ & 3.156 & -0.163 & 0.033 & -12.884 & -0.541 \\
\hline Travel out of UAE & 0.020 & 0.001 & 0.986 & -0.041 & 0.040 \\
\hline Life events & 3.203 & 0.124 & 0.108 & -1.148 & 11.517 \\
\hline
\end{tabular}

Table 7.12 Multiple regression analysis of SF-36 total scores with dummy categorical variables for dialysis sample

\begin{tabular}{|c|c|c|c|c|c|}
\hline \multirow[t]{2}{*}{ Variables } & \multirow{2}{*}{$\begin{array}{l}\text { Std } \\
\text { Error } \\
\text { of } \beta\end{array}$} & \multirow{2}{*}{$\begin{array}{l}\text { Standard } \\
\text { Coefficient } \\
\text { ( } \beta \text { Value) }\end{array}$} & \multirow[t]{2}{*}{$\begin{array}{l}\mathbf{p} \\
\text { value }\end{array}$} & \multicolumn{2}{|c|}{$\begin{array}{l}\text { 95\% Confidence } \\
\text { Interval for } \beta\end{array}$} \\
\hline & & & & $\begin{array}{l}\text { Lower } \\
\text { Bound }\end{array}$ & $\begin{array}{l}\text { Upper } \\
\text { Bound }\end{array}$ \\
\hline Ethnicity dummy 1 (UAE vs Rest) & 4.244 & 0.049 & 0.625 & -6.314 & 10.474 \\
\hline Ethnicity dummy 1 (South Asian vs Rest) & 4.615 & 0.110 & 0.278 & -4.099 & 14.158 \\
\hline Ethnicity dummy 3 (Others vs Rest) & 5.972 & 0.043 & 0.666 & -9.232 & 14.391 \\
\hline Marital dummy 1 (Single vs Rest) & 5.645 & -0.023 & 0.794 & -12.639 & 9.691 \\
\hline Marital dummy 2 (Other vs Rest) & 5.415 & -0.056 & 0.574 & -13.761 & 7.660 \\
\hline $\begin{array}{l}\text { Education dummy } 1 \text { (No schooling VS } \\
\text { Rest) }\end{array}$ & 5.391 & -0.094 & 0.417 & -15.054 & 6.270 \\
\hline Education dummy 2 (Primary vs Rest) & 4.906 & 0.053 & 0.643 & -7.423 & 11.982 \\
\hline Education dummy 3 (Secondary vs Rest) & 4.771 & 0.065 & 0.559 & -6.643 & 12.231 \\
\hline Religion dummy 1 (Christians vs Rest) & 6.027 & -0.011 & 0.927 & -12.476 & 11.363 \\
\hline Religion dummy 2 (Others vs Rest) & 8.036 & -0.070 & 0.523 & -21.037 & 10.750 \\
\hline $\begin{array}{l}\text { Living arrangement dummy } 1 \text { (Live alone } \\
\text { vs Rest) }\end{array}$ & 6.735 & $<0.0001$ & 0.999 & -13.316 & 13.325 \\
\hline $\begin{array}{l}\text { Living arrangement dummy } 2 \text { (Others vs } \\
\text { Rest) }\end{array}$ & 4.196 & 0.011 & 0.905 & -7.797 & 8.802 \\
\hline Employment dummy1 (Part time vs Rest) & 4.431 & 0.123 & 0.273 & -3.884 & 13.644 \\
\hline Employment dummy2 (Retired vs Rest) & 5.142 & -0.151 & 0.126 & -18.086 & 2.256 \\
\hline $\begin{array}{l}\text { Employment dummy3 (Unemployed vs } \\
\text { Rest) }\end{array}$ & 4.932 & 0.055 & 0.573 & -6.970 & 12.538 \\
\hline
\end{tabular}

Table 7.12 showed that none of the dummy categorical variables had any statistically significant contribution in explaining the total scores of the SF-36 in dialysis respondents. 
The adjusted $R^{2}$ value for this model was 0.018 , which indicates that this model accounts for $1.8 \%$ of variation in the total SF36 scores. The multiple regression results in Table 7.13 on the clinical laboratory variables for dialysis sample shows that there was a statistically insignificant model for the SF-36 $(p=0.130)$.

Table 7.13 Multiple regression analysis of total SF-36 scores with the clinical variables for dialysis sample

\begin{tabular}{|c|c|c|c|c|c|}
\hline \multirow[t]{2}{*}{ Variables } & \multirow{2}{*}{$\begin{array}{l}\text { Std } \\
\text { Error of } \\
\beta\end{array}$} & \multirow{2}{*}{$\begin{array}{l}\text { Standard } \\
\text { Coefficient } \\
\text { ( } \beta \text { Value) }\end{array}$} & \multirow[t]{2}{*}{$\begin{array}{l}\mathbf{p} \\
\text { value }\end{array}$} & \multicolumn{2}{|c|}{$\begin{array}{l}95 \% \text { Confidence Interval } \\
\text { for } \beta\end{array}$} \\
\hline & & & & $\begin{array}{l}\text { Lower } \\
\text { Bound }\end{array}$ & $\begin{array}{l}\text { Upper } \\
\text { Bound }\end{array}$ \\
\hline $\mathrm{Hb}$ & 0.113 & 0.020 & 0.830 & -0.199 & 0.248 \\
\hline Serum albumin & 0.366 & 0.042 & 0.656 & -0.561 & 0.888 \\
\hline Pre-dialysis BUN (g/L) & 0.299 & $<0.0001$ & 0.997 & -0.591 & 0.594 \\
\hline Pre-dialysis Creatinine ( $\mathrm{g} / \mathrm{L})$ & 0.008 & 0.250 & 0.021 & 0.003 & 0.033 \\
\hline Urea Reduction ratio & 0.264 & -0.033 & 0.727 & -0.615 & 0.430 \\
\hline Length of time on dialysis & 0.031 & -0.027 & 0.762 & -0.072 & 0.053 \\
\hline
\end{tabular}

The pre-dialysis creatinine level variable was the only significant determinant of SF-36. This means that high serum creatinine level had the strongest contribution among the clinical variables in explaining the total scores of the SF-36 in the dialysis respondents. Other clinical variables did not show any statistically significance. The adjusted $R^{2}$ value for this model was 0.032 , which indicates that this model accounts for $3.2 \%$ of variation in the total scores SF-36.

Another model was generated to determine how much of the total variability in the SF-36 for the dialysis sample was predicted by all the variables that included all the continuous variables, all the dummy variables and the clinical variables for the dialysis sample. The adjusted $R^{2}$ value for this model was 0.136 , which indicates that the model accounts for $13.6 \%$ of variation in the total scores SF-36. 


\subsubsection{Multiple regression analysis for the dialysis sample using QOL Index}

The multiple regression results on the demographic variables showed that a significant model being emerged for QOL Index results $(p=<0.0001)$. Table 7.14 shows the regression co-efficient values of QOL Index too with the demographic variables for dialysis sample.

Table 7.14 Multiple regression analysis of QOL Index total scores with the demographic variables for dialysis sample

\begin{tabular}{|c|c|c|c|c|c|}
\hline \multirow[t]{2}{*}{ Variables } & \multirow{2}{*}{$\begin{array}{r}\text { Std } \\
\text { Error of } \\
\beta\end{array}$} & \multirow{2}{*}{$\begin{array}{r}\text { Standard } \\
\text { Coefficient } \\
(\beta \text { Value })\end{array}$} & \multirow{2}{*}{ p value } & \multirow{2}{*}{\begin{tabular}{|c|}
$\begin{array}{l}\text { 95\% Confidence } \\
\text { Interval for } \beta\end{array}$ \\
Lower Bound
\end{tabular}} & \\
\hline & & & & & $\begin{array}{l}\text { Upper } \\
\text { Bound }\end{array}$ \\
\hline Gender & 0.864 & -0.004 & 0.960 & -1.751 & 1.665 \\
\hline Age & 0.036 & 0.030 & 0.738 & -.060 & .084 \\
\hline Chronic health problems & 0.834 & 0.276 & 0.001 & 1.143 & 4.443 \\
\hline Living in UAE & 0.002 & 0.351 & $<0.0001$ & .004 & .013 \\
\hline Cause of kidney failure & 0.849 & -0.191 & 0.016 & -3.741 & -.385 \\
\hline Travel out of UAE & 0.006 & 0.036 & 0.646 & -.008 & .013 \\
\hline Life events & 0.871 & 0.074 & 0.350 & -.905 & 2.538 \\
\hline
\end{tabular}

Variables such as chronic health problems, living in UAE and cause of kidney failure showed a statistically significant determinant of QOL Index. This means that having another long term condition, living longer in UAE and knowing the cause of kidney failure had the strongest contribution in explaining the total scores of the QOL Index in dialysis respondents. Other variables did not show any statistical significance. The adjusted $\mathrm{R}^{2}$ value for this model was 0.139 , which indicates that this model accounts for $13.9 \%$ of variation in the QOL Index total scores.

The multiple regression results on categorical variable of the dialysis sample using the dummy variables approach for the QOL Index showed statistically insignificant model $(p=$ 0.367). Table 7.15 shows the regression co-efficient values of $Q O L$ Index too with the dummy categorical variables for dialysis sample. The table shows that compared with Arab Nationals, being UAE National were a statistically significant determinant of QOL Index. Moreover, compared with respondents who lived alone or with family and friends, respondents who had other living arrangements had trends toward having lower 
statistically significant of the total scores of $Q O L$ Index. The adjusted $R^{2}$ value for this model was 0.009 , which indicates that this model accounts for $9.0 \%$ of variation in the total scores of the QOL Index.

Table 7.15 Multiple regression analysis of QOL Index total scores with dummy variables for dialysis sample

\begin{tabular}{|c|c|c|c|c|c|}
\hline \multirow[t]{2}{*}{ Variables } & \multirow{2}{*}{$\begin{array}{r}\text { Std } \\
\text { Error } \\
\text { of } \beta\end{array}$} & \multirow{2}{*}{$\begin{array}{l}\text { Standard } \\
\text { Coefficien } \\
\text { t ( } \beta \text { Value })\end{array}$} & \multirow{2}{*}{$\begin{array}{r}\mathbf{p} \\
\text { value }\end{array}$} & \multicolumn{2}{|c|}{$\begin{array}{l}95 \% \text { Confidence } \\
\text { Interval for } \beta\end{array}$} \\
\hline & & & & $\begin{array}{l}\text { Lower } \\
\text { Bound }\end{array}$ & $\begin{array}{l}\text { Upper } \\
\text { Bound }\end{array}$ \\
\hline Ethnicity dummy 1 (UAE vs Rest) & 1.122 & 0.259 & 0.011 & .689 & 5.127 \\
\hline Ethnicity dummy 1 (South Asian vs Rest) & 1.220 & 0.071 & 0.486 & -1.561 & 3.266 \\
\hline Ethnicity dummy 3 (Others vs Rest) & 1.579 & 0.050 & 0.612 & -2.321 & 3.925 \\
\hline Marital dummy 1 (Single vs Rest & 1.492 & -0.004 & 0.965 & -3.017 & 2.886 \\
\hline Marital dummy 2 (Other vs Rest & 1.432 & -0.039 & 0.701 & -3.383 & 2.280 \\
\hline Education dummy 1 (No schooling vs Rest) & 1.425 & -0.021 & 0.859 & -3.072 & 2.565 \\
\hline Education dummy 2 (Primary vs Rest) & 1.297 & 0.027 & 0.816 & -2.263 & 2.867 \\
\hline Education dummy 3 (Secondary vs Rest) & 1.261 & 0.079 & 0.481 & -1.603 & 3.387 \\
\hline Religion dummy 1 (Christians vs Rest) & 1.593 & 0.039 & 0.749 & -2.640 & 3.662 \\
\hline Religion dummy 2 (Others vs Rest) & 2.124 & -0.053 & 0.630 & -5.227 & 3.177 \\
\hline $\begin{array}{l}\text { Living arrangement dummy } 1 \text { (Live alone vs } \\
\text { Rest) }\end{array}$ & 1.781 & -0.072 & 0.456 & -4.853 & 2.190 \\
\hline Living arrangement dummy 2 (Others vs Rest) & 1.109 & -0.171 & 0.076 & -4.181 & .207 \\
\hline Employment dummy1 (Part time vs Rest) & 1.171 & 0.049 & 0.662 & -1.803 & 2.831 \\
\hline Employment dummy2 (Retired vs Rest) & 1.359 & 0.008 & 0.937 & -2.582 & 2.796 \\
\hline Employment dummy3 (Unemployed vs Rest) & 1.304 & -0.010 & 0.916 & -2.716 & 2.442 \\
\hline
\end{tabular}

The multiple regression results on the clinical variables for dialysis sample showed that a statistically insignificant model being emerged for QOL Index $(p=0.464)$. Table 7.16 shows the regression co-efficient values of $\mathrm{QOL}$ Index with the clinical variables for dialysis sample. 
Table 7.16 Multiple regression analysis of QOL Index total scores with clinical variables for dialysis sample

\begin{tabular}{|c|c|c|c|c|c|}
\hline \multirow[t]{2}{*}{ Variables } & \multirow{2}{*}{$\begin{array}{l}\text { Std } \\
\text { Error of } \\
\beta\end{array}$} & \multirow{2}{*}{$\begin{array}{l}\text { Standard } \\
\text { Coefficient ( } \beta \\
\text { Value) }\end{array}$} & \multirow[t]{2}{*}{$p$ value } & \multicolumn{2}{|c|}{$\begin{array}{l}95 \% \text { Confidence Interval } \\
\text { for } \beta\end{array}$} \\
\hline & & & & $\begin{array}{l}\text { Lower } \\
\text { Bound }\end{array}$ & $\begin{array}{l}\text { Upper } \\
\text { Bound }\end{array}$ \\
\hline $\mathrm{Hb}$ & 0.028 & -0.050 & 0.600 & -0.069 & 0.040 \\
\hline Serum albumin & 0.099 & 0.011 & 0.909 & -0.184 & 0.207 \\
\hline Pre-dialysis BUN (g/L) & 0.069 & 0.045 & 0.651 & -0.106 & 0.168 \\
\hline Pre-dialysis Creatinine $(\mathrm{g} / \mathrm{L})$ & 0.002 & 0.126 & 0.235 & -0.002 & 0.006 \\
\hline Urea Reduction ratio & 0.069 & -0.083 & 0.385 & -0.198 & 0.077 \\
\hline Years on dialysis & 0.007 & -0.172 & 0.027 & -0.030 & -.0002 \\
\hline
\end{tabular}

The above table showed that the length of time on dialysis was a statistically significant negative determinant of the QOL Index total scores $(p=0.027)$. This mean that the less time spent on dialysis contributed to better QOL Index total scores. None of the clinical variables were statistically significant determent on the QOL Index total scores. The adjusted $R^{2}$ value for this model was 0.003 , which indicates that this model accounts for $0.3 \%$ of variation in the total scores of the QOL Index.

Another model was generated to determine how much of the total variability in the QOL Index for dialysis sample was predicted by all the variables that included all the continuous variables, all the dummy variables and the clinical variables for the dialysis sample was done. The adjusted $\mathrm{R}^{2}$ value for this model was 0.150 , which indicates that this model accounts for $15.0 \%$ of variation in the QOL Index total scores.

\subsubsection{Multiple regression results for the community sample using SF-36}

The multiple regression results on the demographic variables for the community sample presented in Table 7.17 shows that a significant model has been emerged for the SF-36 results $(p=<0.0001)$. 
Table 7.17 Multiple regression analysis of SF-36 total scores with demographic variables for community sample

\begin{tabular}{|c|c|c|c|c|c|}
\hline \multirow[t]{2}{*}{ Variables } & \multirow{2}{*}{$\begin{array}{r}\text { Std Error } \\
\text { of } \beta\end{array}$} & \multirow{2}{*}{$\begin{array}{r}\text { Standard } \\
\text { Coefficient ( } \beta \\
\text { Value) }\end{array}$} & \multirow{2}{*}{$\begin{array}{r}p \\
\text { value }\end{array}$} & \multicolumn{2}{|c|}{$\begin{array}{l}\text { 95\% Confidence Interval for } \\
\beta\end{array}$} \\
\hline & & & & Lower Bound & Upper Bound \\
\hline Gender & -3.283 & -0.100 & 0.104 & -7.246 & 0.679 \\
\hline Age & 0.215 & 0.148 & 0.022 & 0.031 & 0.399 \\
\hline Chronic health problems & 8.552 & 0.191 & 0.002 & 3.171 & 13.934 \\
\hline Living in UAE & -0.009 & -0.088 & 0.174 & -0.022 & 0.004 \\
\hline Travel out of UAE & -0.025 & -0.058 & 0.345 & -0.078 & 0.027 \\
\hline Life events & 6.169 & 0.143 & 0.018 & 1.085 & 11.253 \\
\hline
\end{tabular}

Variables such as age, chronic health problems and life events showed a statistically significant determinant of QOL Index. This means that being older, having long term condition and major events in the past 12 months had the strongest contribution in explaining the total scores of the SF-36 in community respondents. Other variables did not show any statistical significance. The adjusted $R^{2}$ value for this model was 0.084 which indicates that this model accounts for $8.4 \%$ of variation in the total scores of the SF-36.

The multiple regression results on the categorical variable of the community sample using dummy variables for the SF-36 showed a statistically significant model being emerged $(p$ $=<0.0001$ ). Table 7.18 shows the regression co-efficient values of SF-36 with dummy categorical variables for the community sample. 
Table 7.18 Multiple regression analysis of SF-36 total scores with dummy variables for community sample

\begin{tabular}{|c|c|c|c|c|c|}
\hline \multirow[t]{2}{*}{ Variables } & \multirow{2}{*}{$\begin{array}{r}\text { Std } \\
\text { Error of } \\
\beta\end{array}$} & \multirow{2}{*}{$\begin{array}{r}\text { Standard } \\
\text { Coefficie } \\
\text { nt }(\boldsymbol{\beta} \\
\text { Value) }\end{array}$} & \multirow{2}{*}{$\begin{array}{r}p \\
\text { value }\end{array}$} & \multicolumn{2}{|c|}{$\begin{array}{l}\text { 95\% Confidence } \\
\text { Interval for } \beta\end{array}$} \\
\hline & & & & $\begin{array}{l}\text { Lower } \\
\text { Bound }\end{array}$ & $\begin{array}{l}\text { Upper } \\
\text { Bound }\end{array}$ \\
\hline Ethnicity dummy 1 (UAE vs Rest) & 3.181 & 0.069 & 0.283 & -2.841 & 9.692 \\
\hline Ethnicity dummy 1 (South Asian vs Rest) & 3.056 & 0.165 & 0.039 & 0.310 & 12.348 \\
\hline Ethnicity dummy 3 (Others vs Rest) & 3.505 & -0.015 & 0.840 & -7.613 & 6.195 \\
\hline Marital dummy 1 (Single vs Rest & 2.723 & -0.074 & 0.281 & -8.307 & 2.421 \\
\hline Marital dummy 2 (Other vs Rest & 4.968 & -0.035 & 0.579 & -12.546 & 7.025 \\
\hline $\begin{array}{l}\text { Education dummy } 1 \text { (No schooling vs } \\
\text { Rest) }\end{array}$ & 6.559 & -0.020 & 0.740 & -15.097 & 10.741 \\
\hline Education dummy 2 (Primary vs Rest) & 4.904 & 0.044 & 0.480 & -6.191 & 13.126 \\
\hline Education dummy 3 (Secondary vs Rest) & 2.801 & 0.075 & 0.241 & -2.222 & 8.810 \\
\hline Religion dummy 1 (Christians vs Rest) & 2.857 & -0.052 & 0.529 & -7.430 & 3.826 \\
\hline Religion dummy 2 (Others vs Rest) & 4.551 & -0.052 & 0.407 & -12.747 & 5.180 \\
\hline $\begin{array}{l}\text { Living arrangement dummy } 1 \text { (Live alone } \\
\text { vs Rest) }\end{array}$ & 3.390 & 0.059 & 0.357 & -3.549 & 9.804 \\
\hline $\begin{array}{l}\text { Living arrangement dummy } 2 \text { (Others vs } \\
\text { Rest) }\end{array}$ & 2.970 & 0.134 & 0.047 & 0.088 & 11.788 \\
\hline Employment dummy1 (Part time vs Rest) & 3.037 & 0.334 & $\begin{array}{r}<0.000 \\
1\end{array}$ & 7.483 & 19.445 \\
\hline Employment dummy2 (Retired vS Rest) & 8.113 & -0.053 & 0.386 & -23.022 & 8.936 \\
\hline $\begin{array}{l}\text { Employment dummy3 (Unemployed vs } \\
\text { Rest) }\end{array}$ & 5.136 & 0.142 & 0.031 & 0.995 & 21.228 \\
\hline
\end{tabular}

In comparison with the Arab Nationals, being a South East Asians had a statistically significant determinant of the SF-36 scores. Also, compared with those who lived with family, respondents who lived alone had a statistically significant determinant of the SF-36 scores. Furthermore, compared with full time employment, part time employment and unemployment were a statistically significant determinant of the SF-36 scores. None of the other dummy categorical variables had any statistically significant contribution in explaining the total scores of the SF-36 in community respondents. The adjusted $R^{2}$ value for this model was 0.129 ; this indicates that this model accounts for $12.9 \%$ of variation in the total scores of the SF-36.

Another model was generated to determine how much of the total variability in the SF-36 for the community sample, was predicted by all the variables that included all the continuous variables and all the dummy variables for the community sample. The adjusted 
$R^{2}$ value for this model was 0.175 , which indicates that this model accounts for $17.5 \%$ of the variation in the total scores of SF-36.

\subsubsection{Multiple regression results for the community sample using QOL Index}

The multiple regression results on the demographic variables presented in Table 7.19 show a statistically significant model has emerged $(p=0.004)$, for the QOL Index results.

Table 7.19 Multiple regression analysis of QOL Index total scores with the demographic variables for community sample

\begin{tabular}{|c|c|c|c|c|c|}
\hline \multirow[t]{2}{*}{ Variables } & \multirow{2}{*}{$\begin{array}{r}\text { Std } \\
\text { Error of } \\
\beta\end{array}$} & \multirow{2}{*}{$\begin{array}{r}\text { Standard } \\
\text { Coefficient ( } \beta \\
\text { Value) }\end{array}$} & \multirow{2}{*}{$p$ value } & \multicolumn{2}{|c|}{$\begin{array}{l}95 \% \text { Confidence Interval } \\
\text { for } \beta\end{array}$} \\
\hline & & & & $\begin{array}{l}\text { Lower } \\
\text { Bound }\end{array}$ & Upper Bound \\
\hline Gender & 0.563 & 0.010 & 0.875 & -1.019 & 1.197 \\
\hline Age & 0.026 & 0.214 & 0.001 & 0.034 & 0.136 \\
\hline Chronic health problems & 0.765 & 0.181 & 0.004 & 0.736 & 3.748 \\
\hline Living in UAE & 0.002 & -0.053 & 0.417 & -0.005 & 0.002 \\
\hline Travel out of UAE & 0.007 & -0.058 & 0.352 & -0.022 & 0.008 \\
\hline Life events & 0.723 & 0.034 & 0.571 & -1.014 & 1.834 \\
\hline
\end{tabular}

Variables such as age and chronic health problems were statistically significant determinant of QOL. This means that being older and having long term condition had the strongest contribution in explaining the total scores of the QOL Index in the community respondents. Other variables did not show any statistical significance. The adjusted $R^{2}$ value for this model was 0.048 , which indicates that this model accounts for $4.8 \%$ of the variation in the total scores of the QOL Index.

The multiple regression results on the categorical variable of the community sample using dummy variables approach for the QOL Index presented in Table 7.20 show statistically significant model emerged $(p=<0.0001)$. 
Table 7.20 Multiple regression analysis of QOL Index total scores with dummy variables for community sample

\begin{tabular}{|c|c|c|c|c|c|}
\hline \multirow[t]{2}{*}{ Variables } & \multirow{2}{*}{$\begin{array}{l}\text { Std } \\
\text { Error } \\
\text { of }\end{array}$} & \multirow{2}{*}{$\begin{array}{l}\text { Standar } \\
\text { d } \\
\text { Coefficie } \\
\text { nt ( } \beta \\
\text { Value) } \\
\end{array}$} & \multirow[t]{2}{*}{$\begin{array}{l}\mathbf{p} \\
\text { value }\end{array}$} & \multicolumn{2}{|c|}{$\begin{array}{l}\text { 95\% Confidence } \\
\text { Interval for }\end{array}$} \\
\hline & & & & $\begin{array}{l}\text { Lower } \\
\text { Bound }\end{array}$ & $\begin{array}{l}\text { Upper } \\
\text { Bound }\end{array}$ \\
\hline Ethnicity dummy 1 (UAE vs Rest) & 0.871 & 0.109 & 0.086 & -0.215 & 3.214 \\
\hline Ethnicity dummy 1 (South Asian vs Rest) & 0.837 & 0.253 & 0.002 & 1.020 & 4.316 \\
\hline Ethnicity dummy 3 (Others ss Rest) & 0.959 & -0.045 & 0.540 & -2.477 & 1.300 \\
\hline Marital dummy 1 (Single vs Rest & 0.744 & -0.117 & 0.086 & -2.746 & 0.184 \\
\hline Marital dummy 2 (Other vs Rest & 1.362 & -0.075 & 0.237 & -4.297 & 1.068 \\
\hline Education dummy 1 (No schooling vs Rest) & 1.798 & -0.007 & 0.902 & -3.764 & 3.320 \\
\hline Education dummy 2 (Primary vs Rest) & 1.344 & 0.045 & 0.471 & -1.677 & 3.617 \\
\hline Education dummy 3 (Secondary vs Rest) & 0.757 & 0.098 & 0.119 & -0.307 & 2.673 \\
\hline Religion dummy 1 (Christians vs Rest) & 0.783 & -0.085 & 0.302 & -2.353 & 0.733 \\
\hline Religion dummy 2 (Others vs Rest) & 1.212 & -0.067 & 0.284 & -3.688 & 1.087 \\
\hline $\begin{array}{l}\text { Living arrangement dummy } 1 \text { (Live alone vs } \\
\text { Rest) }\end{array}$ & 0.916 & -0.063 & 0.325 & -2.707 & 0.900 \\
\hline Living arrangement dummy 2 (Others vs Rest) & 0.814 & 0.052 & 0.436 & -0.968 & 2.240 \\
\hline Employment dummy1 (Part time vs Rest) & 0.831 & 0.233 & 0.002 & 0.952 & 4.224 \\
\hline Employment dummy2 (Retired vs Rest) & 2.224 & -0.050 & 0.409 & -6.220 & 2.541 \\
\hline Employment dummy3 (Unemployed vs Rest) & 1.408 & 0.093 & 0.157 & -0.776 & 4.772 \\
\hline
\end{tabular}

Compared with UAE and Arab Nationals, South East Asians had a statistically significant determinant of the QOL Index scores. Moreover, compared with Arab Nationals, South East Asians and other nationalities UAE Nationals had trends toward having higher statistically significant total scores of QOL Index. Compared with married respondents, single respondents from the community sample had trends toward having lower statistically significant total scores of QOL Index. Furthermore, compared with full time employment, part time employment had a statistically significant determinant of the QOL Index scores. None of the other dummy categorical variables have any statistically significant contribution in explaining the total scores of the QOL Index in community respondents. The adjusted $\mathrm{R}^{2}$ value for this model was 0.133 , which indicates that this model accounts for $13.3 \%$ of the variation in the total scores of the QOL Index.

Another model was done to determine how much of the total variability in the QOL Index for the community sample was predicted by all the variables that included all the continuous variables and all the dummy variables for the community sample. The adjusted $R^{2}$ value for 
this model was 0.155 , which indicates that his model was accounted for $15.5 \%$ of variation in the QOL Index total scores.

\subsection{Multivariate Analysis of Variance (MANOVA)}

This section reports on the findings from the MANOVA analysis for both samples. The MANOVA analysis adjusts for the demographic differences in both samples. Given the relatively small sample size of the study, the MANOVA can only be done on the total scores of the tools. Table 7.21 summarises the findings from the MANOVA test for the dialysis sample.

As shown in Table 7.21, considering the SF-36 and the QOL Index as a two-dimensional outcome for the dialysis sample, the long term condition variable was statistically significant in the SF-36 and QOL Index tools, the effected size of the relationship was weak for both tools as indicated by the Partial eta squared $=0.148,0.129$ respectively. Although the education variable was statistically significant in both tools the effected size of the relationship for the education variable was weak for both tools as indicated by the Partial eta squared $=0.146,0.198$ respectively. Even though, the life event and the length of time lived in UAE variables were statistically significant in the SF-36, the effected size of the relationship for the life event variable was weak as indicated by Partial eta squared $=0.059$ and 0.052 respectively. While age variable was statistically significant in the QOL Index, the effected size of the relationship for the age variable was weak for both tools as indicated by Partial eta squared $=0.052$. Whilst the year on dialysis and knowing the cause of kidney failure variables had trends toward being statistically significant in the QOL Index, the effected size of the relationship for the year on dialysis and knowing the cause of kidney failure variables was weak as indicated by Partial eta squared $=0.035$ and 0.043 respectively.

The life event and the length of time lived in UAE variables were statistically significant in the SF-36, the effected size of the relationship for the life event variable was weak as indicated by Partial eta squared $=0.059$ and 0.052 respectively. While the age variable was statistically significant in the QOL Index, the effected size of the relationship for the age variable was weak for both tools as indicated by Partial eta squared $=0.052$. 
Table 7.21 MANOVA tests for dialysis sample

\begin{tabular}{|c|c|c|c|c|c|c|}
\hline Variables & Dependent Variable & Df & $\begin{array}{l}\text { Mean } \\
\text { Square }\end{array}$ & $\mathbf{F}$ & Sig. & $\begin{array}{l}\text { Partial Eta } \\
\text { Squared }\end{array}$ \\
\hline \multirow[t]{2}{*}{ Gender } & SF36 & 1 & 82.449 & 0.291 & 0.591 & 0.003 \\
\hline & Quality of Life Index & 1 & 21.504 & 1.006 & 0.319 & 0.012 \\
\hline \multirow[t]{2}{*}{ Ethnicity } & SF36 & 3 & 182.729 & 0.644 & 0.589 & 0.023 \\
\hline & Quality of Life Index & 3 & 37.854 & 1.771 & 0.159 & 0.060 \\
\hline \multirow[t]{2}{*}{ Marital status } & SF36 & 2 & 31.288 & 0.110 & 0.954 & 0.004 \\
\hline & Quality of Life Index & 2 & 14.354 & 0.671 & 0.572 & 0.024 \\
\hline \multirow[t]{2}{*}{ Religion } & SF36 & 2 & 569.126 & 2.006 & 0.101 & 0.088 \\
\hline & Quality of Life Index & 2 & 13.940 & 0.652 & 0.627 & 0.030 \\
\hline \multirow{2}{*}{$\begin{array}{l}\text { Living } \\
\text { arrangement }\end{array}$} & SF36 & 2 & 106.189 & 0.374 & 0.865 & 0.022 \\
\hline & Quality of Life Index & 2 & 20.164 & 0.943 & 0.458 & 0.054 \\
\hline \multirow[t]{2}{*}{ Employment } & SF36 & 2 & 446.384 & 1.573 & 0.155 & 0.117 \\
\hline & Quality of Life Index & 2 & 16.092 & 0.753 & 0.628 & 0.060 \\
\hline \multirow[t]{2}{*}{ Education } & SF36 & 3 & 673.815 & 2.374 & 0.036 & 0.146 \\
\hline & Quality of Life Index & 3 & 73.230 & 3.425 & 0.005 & 0.198 \\
\hline \multirow[t]{2}{*}{ Life event } & SF36 & 1 & 1473.261 & 5.192 & 0.025 & 0.059 \\
\hline & Quality of Life Index & 1 & 18.231 & 0.853 & 0.358 & 0.010 \\
\hline \multirow{2}{*}{$\begin{array}{l}\text { Long term } \\
\text { condition }\end{array}$} & SF36 & 1 & 4103.569 & 14.460 & $<0.0001$ & 0.148 \\
\hline & Quality of Life Index & 1 & 262.355 & 12.272 & 0.001 & 0.129 \\
\hline \multirow[t]{2}{*}{ Age } & SF36 & 1 & 65.398 & 0.230 & 0.632 & 0.003 \\
\hline & Quality of Life Index & 1 & 97.079 & 4.541 & 0.036 & 0.052 \\
\hline \multirow[t]{2}{*}{ Living in UAE } & SF36 & 1 & 1301.222 & 4.585 & 0.035 & 0.052 \\
\hline & Quality of Life Index & 1 & 49.438 & 2.313 & 0.132 & 0.027 \\
\hline \multirow{2}{*}{$\begin{array}{l}\text { Travel out of } \\
\text { UAE }\end{array}$} & SF36 & 1 & 471.718 & 1.662 & 0.201 & 0.020 \\
\hline & Quality of Life Index & 1 & 2.870 & 0.134 & 0.715 & 0.002 \\
\hline \multirow{2}{*}{$\begin{array}{l}\text { Year on } \\
\text { dialysis }\end{array}$} & SF36 & 1 & 165.778 & 0.584 & 0.447 & 0.007 \\
\hline & Quality of Life Index & 1 & 64.434 & 3.014 & 0.086 & 0.035 \\
\hline \multirow{2}{*}{$\begin{array}{l}\text { Cause of } \\
\text { kidney failure }\end{array}$} & SF36 & 1 & 670.438 & 2.363 & 0.128 & 0.028 \\
\hline & Quality of Life Index & 1 & 80.423 & 3.762 & 0.056 & 0.043 \\
\hline \multirow[t]{2}{*}{ Haemoglobin } & SF36 & 1 & 55.212 & 0.195 & 0.660 & 0.002 \\
\hline & Quality of Life Index & 1 & 30.469 & 1.425 & 0.236 & 0.017 \\
\hline \multirow[t]{2}{*}{ Albumin } & SF36 & 1 & 26.287 & 0.093 & 0.762 & 0.001 \\
\hline & Quality of Life Index & 1 & 3.063 & 0.143 & 0.706 & 0.002 \\
\hline \multirow{2}{*}{$\begin{array}{l}\text { Pre-dialysis } \\
\text { BUN }\end{array}$} & SF36 & 1 & 1.763 & 0.006 & 0.937 & $<0.0001$ \\
\hline & Quality of Life Index & 1 & 0.126 & 0.006 & 0.939 & $<0.0001$ \\
\hline \multirow{2}{*}{$\begin{array}{l}\text { Pre-dialysis } \\
\text { Creatinine }\end{array}$} & SF36 & 1 & 167.171 & 0.589 & 0.445 & 0.007 \\
\hline & Quality of Life Index & 1 & 18.743 & 0.877 & 0.352 & 0.010 \\
\hline \multirow{2}{*}{$\begin{array}{l}\text { Urea reduction } \\
\text { Ratio }\end{array}$} & SF36 & 1 & 70.056 & 0.247 & 0.621 & 0.003 \\
\hline & Quality of Life Index & 1 & 21.802 & 1.020 & 0.315 & 0.012 \\
\hline
\end{tabular}

The year on dialysis and knowing the cause of kidney failure variables had trends toward being statistically significant in the QOL Index, the effected size of the relationship for the year on dialysis and knowing the cause of kidney failure variables was weak as indicated by 
Partial eta squared $=0.035$ and 0.043 respectively. The MANOVA test results for the community sample are presented in Table 7.22.

Table 7.22 MANOVA tests for the community sample for SF36 and Quality of Life Index

\begin{tabular}{|c|c|c|c|c|c|c|}
\hline Variables & Dependent Variable & Df & $\begin{array}{l}\text { Mean } \\
\text { Square }\end{array}$ & $\mathbf{F}$ & Sig. & $\begin{array}{l}\text { Partial Eta } \\
\text { Squared }\end{array}$ \\
\hline \multirow[t]{2}{*}{ Gender } & SF36 & 1 & 366.282 & 1.636 & 0.202 & 0.007 \\
\hline & Quality of Life Index & 1 & 0.766 & 0.046 & 0.831 & $<0.0001$ \\
\hline \multirow{2}{*}{ Ethnicity } & SF36 & 3 & 323.898 & 1.447 & 0.230 & 0.019 \\
\hline & Quality of Life Index & 3 & 134.533 & 8.045 & $<0.0001$ & 0.095 \\
\hline \multirow[t]{2}{*}{ Marital Status } & SF36 & 2 & 285.747 & 1.276 & 0.283 & 0.016 \\
\hline & Quality of Life Index & 2 & 26.651 & 1.594 & 0.192 & 0.020 \\
\hline \multirow[t]{2}{*}{ Religion } & SF36 & 2 & 289.171 & 1.292 & 0.278 & 0.017 \\
\hline & Quality of Life Index & 2 & 9.714 & 0.581 & 0.628 & 0.008 \\
\hline \multirow{2}{*}{$\begin{array}{l}\text { Living } \\
\text { Arrangement }\end{array}$} & SF36 & 2 & 204.928 & 0.915 & 0.472 & 0.020 \\
\hline & Quality of Life Index & 2 & 9.164 & 0.548 & 0.740 & 0.012 \\
\hline \multirow[t]{2}{*}{ Employment } & SF36 & 2 & 731.079 & 3.265 & 0.003 & 0.091 \\
\hline & Quality of Life Index & 2 & 46.798 & 2.799 & 0.008 & 0.079 \\
\hline \multirow{2}{*}{$\begin{array}{l}\text { Education } \\
\text { Groups }\end{array}$} & SF36 & 3 & 99.807 & 0.446 & 0.848 & 0.012 \\
\hline & Quality of Life Index & 3 & 15.758 & 0.942 & 0.465 & 0.024 \\
\hline \multirow[t]{2}{*}{ Life event } & SF36 & 1 & 1425.048 & 6.365 & 0.012 & 0.027 \\
\hline & Quality of Life Index & 1 & 0.137 & 0.008 & 0.928 & $<0.0001$ \\
\hline \multirow{2}{*}{$\begin{array}{l}\text { Long term } \\
\text { condition }\end{array}$} & SF36 & 1 & 1531.643 & 6.841 & 0.010 & 0.029 \\
\hline & Quality of Life Index & 1 & 71.509 & 4.276 & 0.040 & 0.018 \\
\hline \multirow[t]{2}{*}{ Age } & SF36 & 1 & 529.320 & 2.364 & 0.126 & 0.010 \\
\hline & Quality of Life Index & 1 & 121.272 & 7.252 & 0.008 & 0.031 \\
\hline \multirow[t]{2}{*}{ Live in UAE } & SF36 & 1 & 153.669 & 0.686 & 0.408 & 0.003 \\
\hline & Quality of Life Index & 1 & 36.629 & 2.190 & 0.140 & 0.009 \\
\hline \multirow{2}{*}{$\begin{array}{l}\text { Travel out of } \\
\text { UAE }\end{array}$} & SF36 & 1 & 137.816 & 0.616 & 0.434 & 0.003 \\
\hline & Quality of Life Index & 1 & 30.201 & 1.806 & 0.180 & 0.008 \\
\hline
\end{tabular}

Considering the SF-36 and the QOL Index tools as a two-dimensional outcome for the community sample, the long term condition and employment variables were statistically significant in both tools at $p=0.010,0.040$ and $0.003,0.008$ respectively. The effect size of the relationship was weak for both tools as indicated by Partial eta squared $=0.029,0.018$ and $0.091,0.079$ respectively. Moreover, the ethnicity and age variable were statistically significant in the QOL Index only at $<0.0001$ and 0.008 . The effect size of the relationship was weak for the tool as indicated by Partial eta squared $=0.095$ and 0.031 . Other variables did not show any statistical significance. 


\subsection{Conclusion}

The dialysis respondents' overall QOL was rated statistically significantly higher when selfassessed using the QOL Index compared with the SF-36. In contrast, the overall QOL of the community sample was rated slightly higher but statistically insignificant when selfassessed using the QOL Index compared with the SF-36. In the dialysis sample, the mean total score of the PHC and the MHC were lower compared with the community sample. When the QOL Index was used the community sample had statistically significantly better scores on health and functioning and family subscales. The differences in the statistically significant values of both tools in the dialysis and community samples showed contradictory results. Some demographic variables were statistically significant when the SF-36 was used and but were statistically insignificant when QOL Index used.

The differences in the statistically significant values for the multiple regression analysis for both tools in the dialysis and community samples showed contradictory results. In the dialysis sample, the multiple regression analysis on the demographic variables using the SF-36 showed that chronic health problems, knowing the cause of kidney failure and predialysis creatinine levels were statistically significant determinants of QOL. However, when the QOL Index tool was used, years on dialysis and chronic health problems, living in UAE and cause of kidney failure variables were statistically significant determinants of QOL. In the community sample, the multiple regression results on the demographic variables using the SF-36 showed that age, long term condition and life events were statistically significant determinants of QOL. Compared with full time employment, part time employment and unemployed were statistically significant determinants of the SF-36 scores. In comparison, h Arab Nationals, South East Asians had a statistically significant determinant of the QOL Index scores compared with other ethnicities. Furthermore, compared with full time employment, part time employment had a statistically significant determinant on the QOL Index scores.

The findings from the MANOVA analysis for the dialysis and the community samples showed that the presence of a long term condition was also statistically significant in both tools. In the dialysis sample, the education variable was statistically significant in both tools. However, the life event and the length of time lived in UAE variables were statistically 
significant in the SF-36 only. The age variable was statistically significant in the QOL Index only. In the community sample, the employment variables were statistically significant in both tools. However, the ethnicity and age variable were statistically significant in the QOL Index only. The next chapter discuss the findings of this study. 


\section{Chapter 8 Discussion of the findings from the tools}

The first section of this chapter discusses the differences in the findings between the SF36 and QOL Index scores for the samples. Also, this section compares the significant values of the two tools in both samples. This section discusses the findings from the blood results of the dialysis sample. Section two explores the impact of the socio-demographic factors and the clinical variables that contribute to the total scores of both tools for both samples and compares the findings of both samples with international studies. Section three discusses the relationship between the clinical variables and the total scores of both tools in the dialysis sample. Section four explores the comparison of the significant values of the two tools. Section five discusses the demographic and clinical determinants of QOL scores and compares this with international studies. Section six examines the predictors of the QOL scores for both tools.

\subsection{Findings from each QOL tools}

\subsubsection{The findings from the SF-36}

The finding that the community sample had statistically significant lower scores on the body pain, general health and vitality subscales compared with the dialysis sample was unexpected (18.07, 45.25, 52.33 vs $26.07,52.53,55.91$ respectively) as outlined in Table 6.1. Respondents from the community who were less than 50 years old reported higher pain scores compared with the dialysis sample. The community sample was expected to be healthier than the dialysis sample as the majority (83.1\%) of them did not suffer from long term conditions. However, there might be other factors that had an influence on pain such as culture and the psychological status of individuals. Cultural and ethnic groups react to pain differently. Culture influences how a person experiences and responds to pain (Narayan, 2010). Intense episodes of pain can affect attitudes and emotions, therefore the self-assessment of pain may be affected by a person's emotional and psychological state as well as by their ethnicity (Bates, Edwards, \& Anderson, 1993). Several investigators have reported ethnic differences in the regularity of pain associated with chronic medical conditions. For example, higher levels of pain have been reported by African-Americans compared to non-Hispanic Caucasians with several painful conditions, including acquired immune deficiency syndrome (Breitbart et al., 1996) and arthritis 
(Creamer Lethbridge-Cejku \& Hochberg, 1999). Edwards et al. (2001) reported higher levels of pain and disability among African-American relative to Caucasian patients seen in a multidisciplinary pain centre.

In this study the reported mean scores of the body pain subscale in the dialysis sample in males were less than the females (24.34 vs 30.23 respectively). This finding is similar to the community sample where the mean scores of body pain subscale for males were less than the females (14.50 vs 22.70 respectively). These findings contradict several studies that have documented that women have higher prevalence to most pain related conditions (Ghezeljeh, 2010; Lund \& Lundeberg, 2008). Gender is a significant predictor of pain perceptions and coping strategies. Affleck et al. (1999) found that women are more likely to report pain and other symptoms, and to express more distress symptoms than men. Women's coping mechanisms are also different to men. Women seek spiritual help and ask more questions about pain. Additionally, the differences between sexes can be linked to a mix of biological, psychological and socio-cultural factors (Bernardes, Keogh, \& Lima, 2008).

The finding that the dialysis sample had on average lower scores on the role physical and the physical function subscales compared with the community sample were expected because the dialysis respondent have at least one long term condition (kidney failure). Living with a chronic disease impacts negatively on education, employment, caregivers and everyday life (Belasco et al., 2006; Liem, Bosch, Arends, Heijenbrok-Kal, \& Hunink, 2007). In contrast, the high scores for the community sample were for the role physical and social functioning subscales were expected because the community sample were healthier as evidenced by the lower percentage of long term conditions and more able to be involved in social activities. The health functioning status of a patient or a person influences his/her perception of QOL (Cukor et al., 2007). Another comparison with the Kao et al. (2009) study which evaluated the QOL of dialysis patients and a sample from the community from Taiwan showed that the UAE dialysis patients and the community sample had lower scores on the body pain and social functioning subscales. Table 8.1 compares the Kao et al. findings with the UAE study. 
Table 8.1 Comparison between the SF-36 subscales and the total scores of both samples and the results from the Kao et al. (2009) study

\begin{tabular}{|l|r|r|r|r|}
\hline SF-36 Scores (0-100) & $\begin{array}{l}\text { UAE Dialysis } \\
(\mathbf{n = 1 5 0 )} \mathbf{M} \pm \mathbf{S D}\end{array}$ & $\begin{array}{l}\text { UAE } \\
\text { Community } \\
(\mathbf{n = 2 6 4 )} \mathbf{M} \pm \text { SD }\end{array}$ & $\begin{array}{l}\text { Taiwan } \\
\text { Dialysis } \\
(\mathbf{n = 8 6 1 )} \mathbf{M} \pm \mathbf{S D}\end{array}$ & $\begin{array}{l}\text { Taiwan } \\
\text { Community } \\
(\mathbf{n = 1 6 8 8}) \mathbf{M} \pm \mathbf{S D}\end{array}$ \\
\hline SF 36 Physical Function & $54.67 \pm 27.80$ & $78.93 \pm 24.40$ & $47.90 \pm 30.49$ & $92.24 \pm 16.16$ \\
\hline SF 36 Role-Physical & $47.00 \pm 44.70$ & $83.43 \pm 31.10$ & $37.57 \pm 44.41$ & $83.65 \pm 33.27$ \\
\hline SF 36 Body Pain & $26.07 \pm 23.10$ & $18.07 \pm 19.80$ & $67.50 \pm 27.49$ & $84.84 \pm 19.42$ \\
\hline SF 36 General Health & $52.53 \pm 15.50$ & $45.25 \pm 12.30$ & $43.10 \pm 24.07$ & $69.29 \pm 21.27$ \\
\hline SF 36 Vitality & $55.91 \pm 13.70$ & $52.33 \pm 11.60$ & $48.32 \pm 22.43$ & $68.27 \pm 18.66$ \\
\hline SF 36 Social Functioning & $44.58 \pm 18.20$ & $46.88 \pm 13.60$ & $60.96 \pm 27.26$ & $86.81 \pm 17.05$ \\
\hline SF 36 Role Emotional & $55.33 \pm 46.30$ & $76.03 \pm 77.10$ & $49.20 \pm 46.16$ & $79.40 \pm 36.07$ \\
\hline SF 36 Mental Health & $58.75 \pm 12.60$ & $59.14 \pm 10.80$ & $59.04 \pm 21.46$ & $73.01 \pm 16.55$ \\
\hline
\end{tabular}

The community sample from Taiwan scored higher in all subscales compared with the UAE study except the role physical subscale. On the other hand, the UAE dialysis sample scored higher in all subscales except the body pain, social functioning and mental health subscales. These findings also support the argument that people from different cultures and ethnicities perceive and respond to pain differently. It might be that the UAE population in general over-estimate their level of response to pain or may have different concept of pain. Further analysis to the question related to pain on the body pain subscale found that UAE and Arab Nationals had statistically significant lower scores on this question compared with respondents with South East Asia ethnicity on the SF-36 ( $p=$ 0.008). The mean score for the social functioning subscale obtained for both samples was lower than the Taiwan study. It could be speculated that social relations in different countries plays a critical role in individual's daily life. This may reflect the differences in the rating of the SF-36 items regarding the expectations of life between different cultures.

In the dialysis sample, the mean total score of the PHC was 45.1 and the MHC was 53.6. These findings were low compared with the findings from Bohlke et al. (2008) who studied 140 dialysis patients in Brazil. Bohlke and colleagues found that the PHC scale was 57.5 and $\mathrm{MHC}$ was 73.6. On the other hand, the findings from the UAE study are considered relatively high compared with the study by Pakpour et al. (2010), who reported lower scores (41.2) on the PHC and 47.5 on the MHC scales of 250 Iranian dialysis patients. In another comparison with other international studies of the PHC and the MHC scales of the SF-36, the UAE study reported slightly lower scores for the dialysis sample compared with several studies done in the USA, Turkey, Russia and Taiwan as illustrated in Table 8.2. 
Table 8.2 Comparison between dialysis samples of the UAE study with international studies

\begin{tabular}{|c|c|c|c|c|c|}
\hline SF-36 Scores (0-100) & $\begin{array}{l}\text { UAE } \\
(n=150)\end{array}$ & $\begin{array}{l}U^{\prime} A^{\prime} \\
(n=36,582)\end{array}$ & $\begin{array}{l}\text { Turkey" } \\
(\mathrm{n}=75)\end{array}$ & $\begin{array}{l}\text { Russia'"I } \\
(n=1047)\end{array}$ & $\begin{array}{l}\text { Taiwan' } \\
(\mathrm{n}=861)\end{array}$ \\
\hline Physical Health Component (PHC) & 45.10 & 49.80 & 49.84 & 46.87 & 49.02 \\
\hline Mental Health Component (MHC) & 53.60 & 56.17 & 56.88 & 57.10 & 54.38 \\
\hline
\end{tabular}

Sources:

i. Liem, et al. (2007). Quality of life assessed with the Medical Outcomes Study Short Form 36-Item Health Survey of patients on renal replacement therapy: A systematic review and meta-analysis. The Journal of International Society of Pharmacoeconomics and Outcome Research, 10(5), 390-397.

li Sayin, et al. (2007). Quality of life in hemodialysis, peritoneal dialysis, and transplantation patients. Transplant Proceeding Journal, 39(10), 3047-3053.

lii Vasilieva, I. (2006). Quality of life in chronic hemodialysis patients in Russia. Hemodialysis International Journal, 10(3), 274-278.

iv Kao, et al. (2009). Economic, social, and psychological factors associated with health-related quality of life of chronic hemodialysis patients in northern Taiwan: A multicenter study. Artificial Organ Journal, 33(1), 61-68.

The UAE dialysis patients scored slightly lower on the PHC and the MHC and the total score of the SF-36 compared with a major study done by Liem et al. (2007). Involving a meta-analysis on 23 studies in the USA, while the Russian dialysis patients (Vasilieva, 2006) scored the second lowest of the PHC and the Taiwanese dialysis patients (Kao et al., 2009) scored the second lowest on the MHC. The differences in the above findings from different studies could be related to several factors. Firstly, the ethnicity and the culture of the respondents are different. Secondly, the level of social and family support received by respondents varies from one country to another depending on the strength of the family relationships. Thirdly, the severity of other co-morbid conditions associated with dialysis patients might influence the QOL scores (Bakewell et al., 2002).

\subsubsection{Comparison between the SF-36 subscales of the community samples with the normative values from international studies}

Although this study is not representative of the population of the UAE and there were no age gender matched sample, the findings can be considered as regional (Abu Dhabi) baseline data for the dialysis and the community sample in UAE until normative values for the UAE population are obtained. As such, this research can be used for comparisons with specific populations in the same country in future studies. Normative values of the SF-36 for participants from the general population in Turkey and Jordan were reported in the literature. The limitations discussed in Chapter 2 of the Turkey and Jordanian samples in 
their normative studies lend support to this decision. Only these countries' normative values are looked at because they are Middle Eastern Islamic countries. A comparison between the values of this study with the normative values of these countries is presented in Table 8.3.

Table 8.3 Comparison of the normative values of the SF-36 between UAE, Turkey, and Jordan

\begin{tabular}{|l|c|c|c|}
\hline SF-36 Scores $(0-100)$ & $\begin{array}{c}\text { UAE } \\
(\mathbf{n = 2 6 4 )} \\
\mathbf{M} \pm \text { SD }\end{array}$ & $\begin{array}{c}\text { Turkey'. } \\
(\mathbf{n = 1 2 7 9 )} \\
\mathbf{M} \pm \text { SD }\end{array}$ & $\begin{array}{c}\text { Jordan } \\
\mathbf{( n = 5 1 1 )} \\
\mathbf{M} \pm \mathbf{S D}\end{array}$ \\
\hline Physical Function & $78.9 \pm 24.4$ & $83.8 \pm 20.0$ & $66.5 \pm 28.3$ \\
\hline Role-Physical & $83.4 \pm 31.1$ & $86.3 \pm 24.9$ & $60.4 \pm 34.8$ \\
\hline Body Pain & $18.1 \pm 19.8$ & $82.9 \pm 18.9$ & $56.4 \pm 26.7$ \\
\hline General Health & $45.3 \pm 12.3$ & $71.6 \pm 16.1$ & $64.0 \pm 18.5$ \\
\hline Vitality & $52.3 \pm 11.6$ & $64.5 \pm 12.9$ & $55.7 \pm 19.5$ \\
\hline Social Functioning & $46.9 \pm 13.6$ & $91.0 \pm 12.9$ & $66.4 \pm 22.2$ \\
\hline Role Emotional & $76.0 \pm 77.1$ & $90.1 \pm 19.4$ & $58.7 \pm 40.2$ \\
\hline Mental Health & $59.1 \pm 10.8$ & $71.0 \pm 11.0$ & $61.2 \pm 22.2$ \\
\hline
\end{tabular}

i. Altintepe, L., et al. (2006). Physical disability, psychological status, and health-related quality of life in older hemodialysis patients and age-matched controls. Hemodialysis International, 10(3), 260266.

ii. Khader, S., et al. (2011). Normative data and psychometric properties of short form 36 health survey (SF-36, version 1.0) in the population of north Jordan. Eastern Mediterranean Health Journal, 17(5), 368-374.

This table shows that, the regional values of the SF-36 subscales in the UAE study had the lowest scores in five subscales (body pain, general health, vitality, social functioning and mental health) compared with the normative values of the other studies presented, and was second lowest for physical function and role physical. The overall results are closest to those of the Jordanian study. The response rates in the Jordanian sample $(92 \%)$ were similar to the UAE study (93\%). There were small differences in the mean age of both samples (Jordanian 35.8 years, UAE 40.5), and the percentages of male respondents (59.9 compared with 56.9). The majority of both samples were married $(65.2 \%, 74.5$ respectively). The body pain subscale in the UAE sample (18.1), is very low compared with the Jordanian study (56.4), and the Turkish study (82.9). In linking body pain with chronic illnesses, $36.2 \%$ of the Turkish study had one or more chronic illnesses diagnosed by physician compared with $15.4 \%$ of the UAE study. Those participants who had reported a disease had lower scores for all variables. The chronic illnesses variable was not studied in the Jordanian study. Possible explanations for the low score of the body pain subscale 
in the UAE study could be rooted to the culture and way of life and what pain means to the population of the UAE. In Arabic culture, asking people generally about their health may grant a "good" response, but when asking about specific aspects of health a different perspective may be revealed. So, asking specifically about pain may have granted accurate answeres that reflect their perceptions of pain.

The Turkish normative values of the role physical (86.3), social functioning (91.0) and role emotional (90.1) subscales were the highest scores compared with the other studies. The Jordanian and the UAE values of the vitality subscale were lower compared with the Turkish study $(55.7,52.3$, vs 64.5). However, there was less variability amongst the three results on the vitality subscale, but UAE results at 52.3 were still 12.2 points lower than the Turkish study and 3.4 points less than the Jordanian study. This might reflect the differences in the level of physical activities and way of life style among the selected samples in both countries.

\subsubsection{The findings from the QOL Index}

In this UAE study the health and functioning subscale scores were lower and the family subscale scores were higher in the dialysis sample compared with the community sample ( $p=0.001$ and 0.005 respectively). This supports the argument in the conceptual framework that people in Arab and Muslim countries receive considerable family support when they become sick. The total scores of the QOL Index (23.18) for the dialysis samples were in contrast to Parker, Bliwise, Bailey and Rye (2005) who reported lower scores using the QOL Index when studying 16 haemodialysis patients and eight pre-dialysis patients except for the family subscale $(22.5,18.4$ respectively).

\subsubsection{Blood results of the dialysis sample}

Studying QOL in dialysis patients without paying attention to anaemia severity, dialysis adequacy and malnutrition may influence the overall assessment of the QOL. As outlined in Table 7.3 the blood results of the dialysis sample indicated that they have a normal haemoglobin level in the male patients' group and above the therapeutic level in the female group. These findings are supported by the recommendations from the National Kidney Foundation's Kidney Disease Outcomes Quality Initiative (NKF-K/DOQI(TM)) who 
recommended a target haemoglobin range in dialysis patients between 110 to $120 \mathrm{~g} / \mathrm{dL}$ (National Kidney Foundation, 2006). Targeting haemoglobin levels above $130 \mathrm{~g} / \mathrm{L}$ in predialysis or dialysis patients is currently inadvisable (Level 1 evidence) (CARI, 2008).

Anaemia has a negative effect on QOL (Breiterman-White, 2005). Partial correction of anaemia to maintain haemoglobin levels in a target range typically yields significant improvements in both physical and mental health-related QOL in dialysis patients (National Kidney Foundation, 2006). In a study by the Medical Education Institute, patients whose haemoglobin levels fell below $111 \mathrm{~g} / \mathrm{L}$ even for a brief period of time consistently reported deteriorations in their physical function functioning that reduced their ability to perform routine tasks (Schatell \& Witten, 2004). Anaemia negatively influences a broad range of parameters that can decrease functional ability in patients on dialysis such as reduced energy and activity levels, poor sleep and eating behaviour, decline in general health status, impaired sex life, reduce exercise capacity, decreased strength, increased muscle weakness, occasional leg cramps and increased shortness of breath (Breiterman-White), therefore potentially reducing overall QOL.

This study reported that more than half of the dialysis sample had a serum albumin level below the therapeutic range. Other studies have reported that anaemia control and high albumin levels are associated with improved survival and QOL (Bergström \& Lindholm, 1998; Eknoyan et al., 2002; Kimmel \& Patel, 2006; Locatelli et al., 2004; Lopes, et al., 2007). This study reported high urea and creatinine levels above the therapeutic range. This is an expected result due to the inability of the kidney to excrete waste product such as urea and creatinine in case of kidney failure.

Most of the dialysis respondents were well dialysed, the urea reduction ratio was above $65 \%$ in $92 \%$ of the respondents. The impact of dialysis adequacy on the QOL for dialysis patients is still debatable in the literature. In a study done to identify differences between the QOL of people who were adequately dialysed and those inadequately dialysed, Cleary and Drennan (2005) found that differences were presented in the mental health scores of the SF-36 between patients who were well dialysed and those less well dialysed. This finding is supported by the UAE study where the MHC scores of the SF-36 in the dialysis sample were greater than the PHC scores. Furthermore, Manns et al. (2003) and Hamilton 
and Locking-Cusolito (2003) reported that dialysis adequacy was significantly associated with QOL in hemodialysis patients. In contrast, Morton et al. (1996) found that no significant association between dialysis adequacy and any of the domains of QOL.

\subsection{The relationship between the socio-demographic variables and the total scores of both tools in both samples}

The comparison of the findings between the demographic variables and the total scores of both tools for both samples were summarised in Chapter 7 Table 7.4 and 7.7.

\subsubsection{Gender}

The finding that gender variable did not show any statistically significant relationship with the total scores of SF-36 in the dialysis sample was unexpected but is similar to a USA study done by Kalantar-Zadeh et al. (2001) who study 339 hemodialysis outpatients, including 181 men, who were aged $54.7 \pm 14.5$ years. Their samples were selected randomly from seven dialysis units in Los Angeles South/East Bay area. Other studies (Covic et al., 2004; Kutner et al., 2005; Morsch et al., 2006) found that male dialysis patients had higher SF-36 scores. The reason for this gender difference in different studies remains speculative. Possible explanations could include biological factors and biases in the provision of care according to gender (Mustard, Kuafert, \& Kozyrskyj, 1998). Other explanations could be attributed to the effect of differences in clinicians' attitudes toward female patients (Safran, Rogers, Tarlov, McHorney, \& Ware, 1997).

This study found that male respondents in the community sample had statistically significantly higher SF-36 scores compared with female respondents (77.4 vs 71.9 respectively). The higher scores in males from the community sample most probably reflect the differences in men's perceptions of life. It is the researcher's observation that men in the UAE as in other Middle Eastern countries have fewer social restrictions and socialise differently from women. In Middle Eastern culture men are taught to be independent and self-controlled whereas women are brought up to be emotionally expressive and dependent on male members of their families.

The finding that gender variable did not show any statistically significant relationship with the total scores of QOL Index in both samples was different from the SF-36 findings. 
These findings contradict the presumptions in the conceptual framework that male gender in both groups was expected to have better QOL than females due to the cultural factors that restrict women in the UAE such as not being able to live alone, and not having fully independent life. There is no readily available explanation for these findings except that both tools measures QOL differently and therefore gives different results.

\subsubsection{Ethnicity}

The finding that ethnicity did not have any statistically significant effect on the total scores of the SF-36 in the dialysis sample but did for the community sample was unexpected. Respondents with South East Asian ethnicity had higher scores than those in the other two ethnic groups. South East Asians who live in the UAE generally have a poor socioeconomic status compared with UAE Nationals and Arab Nationals. One factor that may contribute to the result is that they usually live in the UAE due to the poor living conditions in their home countries. Ayers, Thomson, Al-Hassiny, Rich and Newton (2008) have found that immigrants generally move out of their countries to improve their QOL. South East Asians had poor living conditions in their home countries; therefore they live better life in the UAE compared with their own countries. No studies were found in the literature comparing the QOL of UAE Nationals, Arab Nationals, South East Asia Nationals and other nationalities. Several studies involving patients who receive hemodialysis in the USA found that African Americans reported higher SF-36 total scores compared with white Americans (Hicks et al., 2006; Kutner \& Devins, 1998; Kutner et al., 2000; Kutner et al., 2005; Lopes et al., 2003). The existence of differences between cultures may illustrate disparities in the management of disease between different countries (Pakpour et al., 2010).

Ethnicity had statistically significant differences in the total scores of the QOL Index in the dialysis sample, favouring people with a UAE nationality $(p=0.023)$. In contrast, the respondents from South East Asia had statistically significant higher total scores on the QOL Index in the community sample $(p=<0.0001)$. UAE national dialysis patients normally receive free medical treatment and other kinds of government aids including free housing, while healthy South East Asians earn better income and live in better living conditions compared with their own home countries. 
The differences in the findings between both tools with regards to ethnicity variable in the dialysis sample could be related to the SF-36 is not being sufficiently sensitive to capture the effect of ethnicity differences on QOL. This finding contradicts the proposition in the conceptual framework that UAE Nationals were expected to have better QOL compared with other ethnic groups because they receive free medical treatment and financial support from the government.

\subsubsection{Marital status}

This study finding that there were no statistically significant differences in marital status and the total scores of the SF-36 in both samples is similar to other studies that have measured the QOL of dialysis patients using the SF-36 (Bohlke et al., 2008; Kao et al., 2009; Merkus et al., 1999). In contrast, this finding differs from Morgan (2009) who found that the quality of marital relationship is a strong predictor of health outcomes than just being married alone, especially when people face great life challenges due to disease complications and associated physical and psychological stressors.

Zarifian (1994) found that dialysis patient had a marked deterioration in their sexual drive and performance. Sexual dysfunction can change the dynamics in a marriage (Palmer, 2003). It can impact negatively on their marital relationship as well as their QOL. The development of a long term condition may place strain on usual family roles and might change patient's ability to work. The amount of support dialysis patients receive from their spouses and the quality of marital relationships is very important in determining how people cope with their illnesses and how they deal with the stressors that accompany living with that long term condition (Cukor et al., 2007). The degree of support received within the family environment has been described as an important predictor of the QOL among dialysis patients (Maor et al., 2001).

The finding that marital status did not have any statistically significant influence on the total scores of the QOL Index in the dialysis sample and had a statistically significant influence in the community sample ( $p=0.690,0.020$ respectively) favouring married respondents. Possible explanation for the differences in the QOL Index findings and the SF-36 is that the SF-36 is not sensitive in capturing the relationship between marital status and QOL. This finding has not been reported in the literature; therefore further research is needed to 
explore the impact of marital status on the QOL using the QOL Index in dialysis and community samples.

\subsubsection{Living arrangements}

The finding that living arrangements did not have any statistically significant influence on the total scores of the SF-36 in the dialysis sample is contrary to the finding in the community sample favouring the group who had other living arrangements such as living with friends. In the UAE people who live with friends are usually young and not married, so they have more freedom from family responsibilities compared with married couples. To the best of my knowledge, this is the first study that reports on the link between living arrangements and QOL in ESRD patients and community samples using the SF-36.

The finding on the QOL Index tool that the living arrangements for dialysis patients who have other living arrangement had a statistically significant lower total scores of 21.4 ( $p=$ 0.038 ) compared with dialysis patients who live alone or who live with family members is contradicting the findings from the community sample. This could indicate that dialysis patients who have other living arrangement lack the required support from their family members. However, people who live with patients who have long term conditions are exposed to more life stressors because they have extra responsibilities towards them. The relationship between the living arrangements and the total scores of the QOL Index has not been reported in the literature; therefore further research is needed to explore this issue in more detail.

\subsubsection{Employment}

The present study showed that having full-time employment had statistically significant positive influence on the SF-36 total scores in both samples ( $p=0.009,<0.0001$ respectively). The finding that the dialysis respondents who were disabled or retired scored 15.4 points lower than those who were employed in full-time jobs and the unemployed group scored 11.7 points higher than the retired and disabled patients is likely related to the difference in the severity of illness as measured by the time on dialysis. Those in the retired and disabled group have been on dialysis for an average of 55 months compared with those in the unemployed group who had been on dialysis for an average of 47 months. The retired and disabled dialysis respondents were on average three years 
younger than the unemployed respondents. Another factor that may contribute to unemployment is the access to dialysis services. In the UAE almost all facilities offer dialysis treatments during the daytime only, making it difficult for hemodialysis patients to maintain a normal working hours. Employers are usually reluctant to employ workers on dialysis due to frequent absences from work in order to go for a medical follow-up. Even patients who were employed had been forced to take either lower paid jobs or lose their jobs after going on dialysis (Ferrans \& Powers, 1993). Kao et al. (2009) reported that work status was associated with higher QOL scores. In contrast, Bohlke et al. (2008) reported lower scores on the SF-36 among dialysis patients who were employed. The financial hardship resulting from losing jobs can mean patients have to change their life style and such changes can affect their ability to maintain social relationships (Ferrans, \& Powers; Wingate, 1995). Full-time employment in dialysis patients may add physical and emotional stress as it requires extra strength and stamina, which some dialysis patients may not have. Studies have found that factors such as dialysis duration (van Manen et al., 2001), employment, physical functioning ability, and co morbidities were associated with the work status of patients undergoing dialysis treatment (Molsted et al., 2004).

The finding that employment did not significantly impact on the total scores of the QOL Index in the dialysis sample contrasts with the findings in the community sample. The community sample who worked full-time had statistically significantly higher scores compared with other samples on the QOL Index $(p=0.001)$. As reported in Chapter 5 Table $5.5,37.3 \%$ of the dialysis sample had full-time employment compared with $79.8 \%$ from the community sample. This is the first known study that compares the employment status of dialysis patients and a community sample using the QOL Index.

\subsubsection{Education}

The finding that educational level variable did not have bearing on the total scores of the SF-36 in both samples was not expected and differs from the findings from other studies. Other studies have linked higher educational level with better QOL (Kao et al., 2009; Lopes et al., 2007; Manns et al., 2003; Moreno et al., 1996). Furthermore, Acaray and Pinar (2005) reported that most of QOL dimensions in the SF-36 increased as educational status increased. Also, the above findings were confirmed by Suet-Ching (2001) who studied the QOL in 164 Hong Kong dialysis patients using Chinese Dialysis QOL Scale. 
Furthermore, Moreno et al. (1996) reported the same findings when studing QOL in 1013 randomly selected stable Spanish dialysis patients in multicentre study using the Karnofsky Scale and the Sickness Impact Profile. It was expected that higher levels of education would positively promote healthy behaviours, and highly educated dialysis patients may volunteer to take some responsibilities of their own health and learn some strategies to cope with their disease and its symptoms, resulting in better QOL (Mingardi et al., 1999; Moreno et al.; Neto et al., 2000). It was expected that educated UAE dialysis patients were having more information about their illness and might have better coping skills. However, chronic kidney failure impacts on all aspects of life and education level is just one of them.

The finding that the education variable did not have any statistically significant influence on the total scores of the QOL Index in both samples is supported by Mozes, Shabtai and Zucker (1997) who studied the differences in QOL among 680 patients receiving dialysis replacement therapy at seven medical centres in USA using the QOL Index tool. Mozes et al. linked higher educational level and acquired skill used at work with the ability to adjust to physical incapability.

\subsubsection{Religion}

Although Christian respondents in both samples scored higher in the SF-36 than Muslims and respondents who believed in other religions, the scores were statistically insignificant in both samples. This finding in the dialysis sample contradicts the finding from a study done by Patel and colleagues (2002) in which they found that there was a relationship between high scores on spiritual beliefs scale, global QOL measures and satisfaction with life in 53 dialysis patients. No studies were found in the literature that had similar religious groupings compared to UAE study. However, spirituality has been examined in a few studies that have explored the QOL of dialysis patients. Kimmel et al. (2003) and Patel et al. (2002) suggested that there is a positive relationship between scores on a spiritual beliefs scale and global QOL measures, satisfaction with life and perception of depression. Furthermore, Berman et al. (2004) found that there is a relationship between religious beliefs and satisfaction with life as well as between organised religious activity and satisfaction with medical care in dialysis patients. Greater perception of spirituality and religiosity has been linked to increased perception of social support and QOL and less 
negative views of illness effects and depression (Patel et al.; Spinale et al., 2008). Religious beliefs impact on a dialysis patient's ability to cope (Ko et al., 2007).

The finding that Christian respondents from the community sample had a statistically significant higher score on the QOL Index $(p=0.020)$ contrasts with the findings from the dialysis sample in which religion variable did not have any bearing on the total scores of QOL Index.

\subsubsection{Life events}

The finding that having major life events in the last 12 months variable did not have any statistically significant effect on the total scores of the SF-36 in the dialysis sample, but had a statistically significant effect in community sample, is likely to be related to the fact that dialysis patients are suffering continuously from a major life event which is kidney failure and they live the associated consequences and complication on a daily basis. Having an additional life event is not likely to have a major impact on their already distorted QOL. Conversely, the community sample are generally healthy, having a major life event such as death of close relatives or being diagnosed with long term condition may have a dramatic impact on their QOL.

The finding that having a major life event in both samples did not have any statistically significant influence on the QOL Index total scores for both samples differs from the finding in the SF-36 with regards to the community sample. This finding might reflect that those tools measures QOL differently. These findings did not support the psycho-social nature of the QOL Index tool that measures a variety of different factors that may influent the satisfactions with and the importance of these factors on QOL. Unlike the SF-36 that normally measures health related issues. The types of life events were not examined in this study; this might be considered as one of the limitations of this study.

\subsubsection{The length of time lived in the UAE and the last time respondents travelled outside the UAE}

The length of time lived in the UAE and the last time respondents travelled outside the UAE did not have any statistically significant correlation with the total scores of the SF-36 in both samples. It was expected that people who lived longer in the UAE had a well 
established life therefore had better QOL compared with those people who lived in UAE for shorter period of time.

The finding that the length of time lived in the UAE had a statistically significant positive correlation with total scores of QOL Index in the dialysis sample ( $p=0.003$ ) was opposite to the findings from the SF-36. The length of time lived in the UAE did not correlate with the total scores of the QOL index in the community sample. Dialysis patients and people from the community who lived for a long time in the country are likely to have a wellestablished social network of friends and family, so they receive more social and family support. The last time respondents travelled outside the UAE did not have any statistically significant correlation with the total scores of the QOL in both samples. It was expected that people who travelled recently might have a chance to see family members or friend and therefore have better QOL. In contrast, people who had major life events in the last 12 months may have some limitations on their ability to travel outside the country. Older people tend to travel less frequently compared with younger people.

\subsubsection{Age}

The finding that age did not have any statistically significant correlation with the total scores of the SF-36 in the dialysis sample is contradicting the finding by Bohlke et al. (2008) who found that higher SF-36 scores were associated with younger age. Advanced age has been linked with the deterioration of physical activity and consequently had lower SF-36 total scores in dialysis patients. In contrast, Valderrábano, Jofre and López-Gómez (2001) reported that older patients were more satisfied with their life on dialysis and accept their limitations better than younger patients. The finding that age did not have any statistically significant correlation with the total scores of the QOL Index for the dialysis sample differs from the finding by Greene (2005) who used the same tool and found that some of the QOL Index scores increased as age increased. They suggested that older chronically ill patients tend to exhibit a greater level of comfort with their health and social status.

The finding that age variable had a statistically significant positive correlation with the total scores of SF-36 in the community sample $(p=0.045)$ is similar to the finding in the QOL Index for the community sample $(p=0.008)$. The possible explanation for these two results 
can be related to the fact that as people grow older some of them achieve what they want in life, such as owning a house, having a well-established job, more financial security and family. In contrast, people at a younger age are still in the process of achieving their objectives in life and building their future. The QOL Index captures the ability to take care of family responsibilities and usefulness to others. Older people sometimes have a decline in their abilities to look after themselves and mainly are dependent on for their others everyday activities.

\subsection{The relationship between the clinical variables and the total scores of both tools in the dialysis sample}

\subsubsection{Chronic health problems}

This study found that those who have another long term condition from the dialysis sample and those who have an ongoing long term conditions in the community sample had lower SF-36 total scores. This is expected because kidney failure impacts negatively on patients' physical, psycho-social and economic wellbeing (Welch \& Austin, 2001). Co-morbid medical conditions are common in patients on dialysis, and are an important contributing factor to clinical outcomes and QOL. Associated diseases, especially diabetes mellitus, are strongly related to the worst QOL scores in ESRD patients on dialysis (Bakewell et al., 2002; Kalantar-Zadeh et al., 2001). In an international comparison of co-morbidity burden, it was found that the USA dialysis population had a significantly higher frequency of these co-morbidities than patients in Europe, with many patients affected by multiple disease processes (Goodkin et al., 2003). Moreover, several health co-morbidities had significant contributions to lower scores of QOL (Lopes et al., 2007). Although, respondents were asked to provide details of their long term conditions, none of them listed any long term condition.

Having a long term condition had a statistically significant influence on the total scores of the QOL Index in the community sample $(p=0.001)$. Long term conditions are normally associated with pain and restrictions in normal daily activities as well as interfere with normal life. Consequently, it has a negative effect on the QOL from the community. Having chronic health problems had trended toward statistically significant influence on the total scores of the QOL Index in the dialysis sample $(p=0.058)$. Long term conditions are 
considered as important contributing factors to clinical outcomes and QOL (KalantarZadeh et al., 2001). Co-morbid diseases influence QOL because they confer disability not associated with renal disease. For example, diabetes is associated with other complications, such as blindness and vascular disease, which cause severe functional limitations (Julius et al., 1989).

\subsubsection{Knowing the cause of kidney failure}

In this study, knowing the cause of kidney failure had a statistically significant contribution to higher scores on the SF-36. This is supported by Pakpour and colleagues (2010) who found that minimal patient knowledge of the disease was one of the determinants of the SF-36 scores. Knowing the cause of kidney failure is linked with the knowledge of the disease. This result supports those of Tsay, Lee and Lee (2005) who linked understanding the disease process and the ability to cope with long term conditions with better QOL scores. Knowing about the illness can help dialysis patients understand the disease process better and helps them in finding ways to decrease the pain and other associated symptoms (Lindqvist et al., 2000). Patients with kidney failure have to take multiple medications and follow strict diet regime. Knowing more about their disease helps them cope with it and improve their QOL. SF-36 measures the functional abilities of the respondents. Therefore, respondents who knew the cause of their kidney failure were more able to cope with their functional limitations.

This study has shown that there are no statistically significant influences of patients knowing the cause of their kidney failure, on the total scores of QOL Index. This finding contradicts other studies that highlighted that pre-dialysis psycho-educational interventions enhance illness-related knowledge (Mehrotra et al., 2005) that promote coping and improve compliance as well as promote QOL (Fukuhara et al., 2006). There was no predialysis education programme available at SKMC. Therefore, most of the dialysis respondents did not have any kind of formal education about the pre-dialysis psychological preparation for dialysis.

\subsubsection{Length of time on dialysis}

In this study, the finding that length of time on dialysis did not have any statistically significant correlation with the total scores of the SF-36 is contradicted to the findings by 
Bohlke et al. (2008) who reported higher scores on the SF-36 among patients who had been on dialysis for shorter lengths of time. In contrast, Morsch et al. (2006) found that patients who had been receiving hemodialysis for more than one year had better QOL scores than patients who had been on hemodialysis for less time. Dialysis treatment removes excessive fluids and uremic toxins from the body which makes patients feels better and therefore improves their QOL (Morsch et al.).

This study also found no statistically significant correlation between the length of time on dialysis and the QOL Index total scores. A review of the literature found no studies reporting any correlation between the length of time on dialysis and the total scores of the QOL Index in dialysis patients. Other studies linked the length of time on dialysis with the development of other co-morbid conditions such as cardiovascular diseases, as well as the myocardial muscle, hyperparathyroidism, renal bone disease and dialysis amylidosis (Drueke \& Eckardt, 2002; Foley et al., 1998; Jimenez et al., 1998; Qunibi, 2004). Hemodialysis normally removes small molecular weight of waste products such as urea and creatinine. Middle molecules such as $\beta 2$-microglobulin and phosphorus are poorly removed by hemodialysis. Accumulation of phosphorus causes calcium phosphorus imbalance and skin itching (Manenti, Tansinda, \& Vaglio, 2009). Accumulation of the $\beta 2-$ microglobulin leads to dialysis amylidosis and the associated pathology tends to increase in severity with time on dialysis (Jimenez et al., 1998).

\subsubsection{Correlation between the total scores of both tools and blood results}

The findings from the correlation tests between the total scores of the SF-36 and the collected laboratory values were presented in Chapter 7 Table 7.6 and 7.9. It was shown that the only laboratory value that correlated significantly with better scores on the total scores of the SF-36 was the pre dialysis creatinine level $(p=0.002)$. Santos and Kerr (2008) found that the body pain subscale score of 59 correlates positively correlated with serum creatinine level $(p=0.009)$. High creatinine level before dialysis could be explained by two reasons. First, higher creatinine before dialysis is usually associated with long intervals between dialysis sessions which indicate sub-optimal dialysis (Locatelli et al., 2002). Second, high creatinine is associated with sufficient food intake, so this could reflect good nutritional status among the dialysis sample. However, other nutritional markers are considered more accurate in assessing the nutritional status in dialysis patients such as 
pre-albumin, transferring concentrations (Rambod et al., 2009). The finding that serum albumin results in this study did not correlate with the total scores of the SF-36 is similar to the finding in a multi-centre study performed by Mingardi et al. (1999). Mingardi et al. reported also no correlation between haemoglobin levels and SF-36 scores. This was attributed to the limited variability of haemoglobin levels in their patients due to the routine use of erythropoietin. Conversely, other studies suggest that anaemia control and high albumin levels were associated with improved survival and QOL (Bergström \& Lindholm, 1998; Eknoyan et al., 2002; Kimmel \& Patel, 2006; Locatelli et al., 2004; Lopes et al., 2007).

In this study, dialysis adequacy, as measured by urea reduction ratio, did not have any statistical correlation with the SF-36 scores. Several studies using other tools have found a correlation between dialysis adequacy with QOL (Bergström \& Lindholm, 1998; Eknoyan et al., 2002; Kalantar-Zadeh et al., 2001; Kimmel \& Patel, 2006; Locatelli et al., 2004; Lopes et al., 2007; Mittal et al., 2001). As outlined in Chapter 1, 10\% of hemodialysis patients in UAE were only on twice weekly dialysis. This study did not consider the frequency of dialysis treatments and the type of dialysers as independent variables. This can be considered one of the limitations of the study. Unruh et al. (2004) and Dwyer et al. (2002) found that the effects of dialysis adequacy measured on the SF-36 were very small. Furthermore, Tsuji-Hayashi et al. (2001) compared the QOL of dialysis patients in Seattle (USA) with dialysis patients in Aichi (Japan). They attributed the better perception of QOL in Japanese patients to improved dialysis adequacy. Lockridge et al. (1999) suggested that the QOL changes experienced in association with changing from conventional hemodialysis to daily dialysis occur in a relatively short period of time.

The correlation between the total scores of the QOL Index and the collected laboratory values were presented in Chapter 6 Table 6.8. It was shown that none of the laboratory values had a statistically significant correlation with the total scores of QOL Index. In contrast, Owen, Lew, Liu, Lowrie and Lazarus (1993) found that serum albumin concentration was highly correlated with better QOL scores among dialysis patients and found to be a strong predictor of mortality. In this study, the mean URR was $75 \%$ and this exceeds the minimum target suggested by the NKF-K/DOQI (2006) guidelines which is $65 \%$, and still did not make a statistically significant correlation with the total scores of the 
QOL Index. The finding that the mean URR of $75 \%$ did not have any statistically significant correlation with the total scores of QOL in both tools contradicts the findings from Manns et al. (2002) study on 128 patients who had been on hemodialysis for more than six months. Manns et al. concluded that patients with above the average URR had higher SF-36 scores. The minimum target URR suggested by the NKF-K/DOQI (2006) is 65\%.

\subsection{Comparison of the significant values of the two tools}

As outlined in Chapter 7 Table 7.10, there are differences in the results between the significant values for the outlined variables in both tools. In the dialysis sample, employment and knowing the cause of kidney failure variables were statistically significant when SF-36 was used. In contrast, ethnicity, living arrangements and length of time on dialysis were statistically significant when QOL Index was used. While in the community sample, gender, living arrangements, Life event and age variables were statistically significant when SF-36 was used. On the contrary, marital status and religion variables were statistically significant when QOL Index was used. These differences could be related to several reasons. Firstly, the QOL Index is disease-specific while the SF-36 is a general tool that was primarily designed to measure health and health-related QOL (Andresen \& Meyers, 2000; Moons, 2004). Secondly, the SF-36 is a health-related QOL tool that mainly measures health or a person's functional ability (Andresen \& Meyers). The SF-36 measures impairment, disability and handicap as well as the ability to perform a task and the performance of tasks (Carr, Thompson, \& Ktrwanf, 1996; Nicole \& Harada, 1999). So, the statistically significant variables (employment and cause of kidney failure) mainly representing the physical and functional abilities of dialysis patients.

Thirdly, the QOL Index measured the respondents' perceptions about their health and life in general (Ferrans \& Powers, 1993). The QOL Index measures satisfaction and importance of sexuality, spirituality, relationships, and self-efficacy (Ferrans \& Powers), therefore it has the benefit of measuring how functional change affects life rather than just measuring functional change. The QOL Index focuses on the impact of disease on the individuals while SF-36 focuses on the functional ability. The QOL Index measures the perception of the QOL in terms of satisfaction with certain aspect of life and the importance of those aspects to the individuals (Ferrans \& Powers). The three statistically significant variables ethnicity, living arrangements, and the length of time lived in the UAE do not 
represent health or functional abilities; they represent a life style factor and the way of living. Therefore, the respondents rated their QOL differently when the two QOL tools were trialled on the same population.

There are few differences in the components of the subscales of both tools. A summary of the subscales addressed by each tool are summarised in Table 8.4.

Table 8.4 Comparison of the subscales in both tools

\begin{tabular}{|l|r|r|}
\hline Subscales all tools & SF-36 & QOL Index \\
\hline Health & Yes & Yes \\
\hline Functioning & Yes & Yes \\
\hline Social & Yes & Yes \\
\hline Economic & Yes & Yes \\
\hline Psychological & Yes & Yes \\
\hline Body Pain & Yes & No \\
\hline Vitality & Yes & No \\
\hline Mental Health & Yes & No \\
\hline Spiritual & No & Yes \\
\hline Family & No & Yes \\
\hline
\end{tabular}

From the above table it can be seen that spirituality and family subscales were not part of the SF-36 and body pain, vitality and mental health were not part of the QOL Index. The rest of the subscales were addressed in both tools. Some subscales were presented in all of them such as physical and mental health subscales. On the other hand, other subscales were presented in one tool. Furthermore, those tools have different scoring systems. In general, the type of questions and the classifications of the subscales were different in both tools.

Although the SF-36 can be used to assess patients on dialysis, there is a question about its ability when it comes to its sensitivity to changes in health conditions of dialysis patients. Disease-specific tools such as the QOL Index, however, may lack some of the comprehensiveness of the generic tools and therefore may not address all issues relating to QOL (Amarantos et al., 2001). The differences in the significant values indicated that each tool captures different aspects of the QOL. The SF-36 measures objective QOL, i.e., health states, capabilities, and functioning, while the QOL Index measures self-reported subjective QOL, i.e., satisfaction with these states, capacities, and functioning. For example, one physical functioning item of the SF-36 asks 'Does your health limit you in 
bathing and dressing yourself?', whereas the QOL Index asks, 'How satisfied are you with your ability to take care of yourself without help? The differences in the scores on the two scales may reflect that if patients adapt or coped well with their illnesses, they can overcome their functional status and their disability (as measured by the SF-36) and live a satisfying life (as measured by the QOL Index). This could be accounted for by the wellknown 'disability paradox' in the QOL research (Albrecht, \& Devlieger, 1999).

The comparison between the significant values of both tools on the same samples revealed differences in the significant values of the total scores of both tools. This finding is supported by Huang et al. (2006) who compared the psychometric properties and factor structures of the SF-36 and World Health Organisation QOL Brief tool (WHOQOL-BREF) on a sample of 11,440 people from Taiwan. They concluded that each tool measures different constructs: the SF-36 measures health-related QOL, while the WHOQOL-BREF measures global QOL.

\subsection{Key demographic and clinical determinants of QOL scores}

Variables that were statistically significant determinants of the total scores of both tools are summarised in Table 8.5.

This table shows that variables relating to having another chronic health problem and knowing the causes of kidney failure were statistically significant determinants of the QOL scores for dialysis respondents on both tools. These findings are supported by Pakpour et al. (2010) who performed a logistic regression studied on the SF-36 Persian version on a sample of 250 Iranian haemodialysis patients. Kalantar-Zadeh et al. (2001) found that the presence of other co-morbid medical conditions are common in patients on dialysis, and are the main contributing factor to clinical outcomes and the QOL. Furthermore, The Hemodialysis (HEMO) Study, which is a 15-centre randomised clinical trial on the effects of hemodialysis dose and membrane flux on mortality and morbidity in patients treated with chronic hemodialysis, showed that the strongest predictor of QOL was coexisting medical conditions. There was a $37 \%$ increase in risk per 1-unit increment in the score on the index with coexisting disease (Unruh et al., 2004). In addition, Bohlke et al. (2008) study of 140 Brazilian dialysis patients found that co-morbidity was the main predictor of the QOL scores of the PHC of the SF-36. 
Table 8.5 Significant variables in multiple regression analyses by tool and sample

\begin{tabular}{|c|c|c|c|c|c|c|c|c|}
\hline & \multicolumn{4}{|c|}{ SF-36 } & \multicolumn{4}{|c|}{ QOL Index } \\
\hline & \multicolumn{2}{|c|}{ Dialysis } & \multicolumn{2}{|c|}{ Community } & \multicolumn{2}{|c|}{ Dialysis } & \multicolumn{2}{|c|}{ Community } \\
\hline Variables & $\begin{array}{l}\beta \\
\text { value }\end{array}$ & Sig. & $\beta$ value & Sig. & $\begin{array}{l}\beta \\
\text { value }\end{array}$ & Sig. & $\begin{array}{l}\beta \\
\text { value }\end{array}$ & Sig. \\
\hline Chronic health & 0.399 & $\begin{array}{l}<0.00 \\
01\end{array}$ & 0.276 & 0.001 & 0.276 & 0.001 & 0.181 & 0.004 \\
\hline Cause of kidney failure & -0.163 & 0.033 & $\mathrm{~N} / \mathrm{A}$ & $\mathrm{N} / \mathrm{A}$ & $-\overline{0}$ & 0.016 & & \\
\hline Pre dialysis creatinine & 0.250 & 0.021 & $\mathrm{~N} / \mathrm{A}$ & $\mathrm{N} / \mathrm{A}$ & & & & \\
\hline Years on dialysis & & & $\mathrm{N} / \mathrm{A}$ & $\mathrm{N} / \mathrm{A}$ & $-\overline{0} 172$ & 0.027 & & \\
\hline Age & & & 0.148 & 0.022 & & & 0.214 & 0.001 \\
\hline $\begin{array}{l}\text { Ethnicity: (South East } \\
\text { Asian vs. Rest) }\end{array}$ & & & 0.165 & 0.039 & & & 0.253 & 0.002 \\
\hline $\begin{array}{l}\text { Ethnicity (UAE vs. } \\
\text { Rest) }\end{array}$ & & & & & 0.259 & 0.011 & & \\
\hline Life events & & & 0.143 & 0.018 & & & & \\
\hline $\begin{array}{l}\text { Living arrangement } \\
\text { (Others vs. the Rest }\end{array}$ & & & 0.134 & 0.047 & & & & \\
\hline $\begin{array}{l}\text { Employment: (Part- } \\
\text { time vs. Rest) }\end{array}$ & & & 0.334 & $\begin{array}{l}<0.00 \\
01\end{array}$ & & & 0.233 & 0.002 \\
\hline $\begin{array}{l}\text { Employment: } \\
\text { (Unemployed vs. Rest) }\end{array}$ & & & 0.142 & 0.031 & & & & \\
\hline Living in UAE & & & & & 0.351 & $<0.0001$ & & \\
\hline
\end{tabular}

The finding that the chronic health illness and the causes of kidney failure variables in the dialysis sample had statistically significant determinants of the QOL Index total scores is consistent with the findings from the SF-36. Furthermore, the Living in UAE and the years on dialysis variables had statistically significant determinants on the QOL Index total scores. The UAE study found that the longer years on dialysis variable had a statistically significant determinate on the QOL Index total scores but it did not have any statistically significant determinate on the QOL when the SF-36 was used. These results are comparable with the HEMO study in which Unruh et al. found that there was a $4 \%$ increase in risk of death per additional year of dialysis. Also, these findings are similar to Bohlke et al. (2008) who found that the length of time on dialysis was the main predictor of the QOL scores of the PHC of the SF-36.

In the community sample, the chronic health problems variable also had statistically significant determinants on the QOL Index total scores. Compared with Arab nationalities, South East Asians from the community sample had a statistically significant determinates 
of QOL SF-36 score. As explained earlier South East Asians in the UAE live a better life compared with home countries. Furthermore, life events variable had statistically significant determinants of the SF-35 scores for respondents from the community sample. It is expected for people who have not any major life events such as death of close relatives to have better QOL scores. This study has shown that compared with people who live with family, people who have other living arrangement had a statistically significant determinates of QOL SF-36 score. Respondents who had other living arrangement represent quarter of the dialysis sample and $15.7 \%$ of the community sample. Other living arrangement means living with friends or living with parents. These categories of respondents were mainly single, young, possibly had less stress and fewer social responsibilities. Compared with full-time employed, respondents who were housekeeper, student, part-time employed or unemployed scored lower on the SF-36 and had a statistically significant determinates of QOL SF-36 score. The unemployed group represents $17.3 \%$ of the dialysis sample and $4.5 \%$ of the community sample. These figures were relatively small compared with respondents who worked full-time from both samples.

The finding that the chronic health illness variable was a statistically significant determinant on the QOL Index total scores in the community sample is consistent with the finding from the dialysis sample using SF-36. The presence of long term condition impacts negatively on the QOL of healthy and non-healthy people. Age variable had statistically significant determinants on the QOL Index and the SF-36 total scores. However, age variable did not have any statistically significant determinant of the total scores for both tools in the dialysis sample. In contrast, Tsay and Healstead (2002) regression analysis in 160 Taiwanese dialysis patients found that only age was significantly related to QOL using QOL Index. Compared with full-time employment, part-time employment from the community sample had a statistically significant determinates on QOL Index total score. But in the dialysis sample employment variable did not have any statistically significant influence on the total scores of the QOL Index. In contrast, Mazes, Shabtai and Zucker (1997) found that age, occupation, educational level, and co-morbid status (mainly diabetes and stroke and ischemic heart disease) were the main risk factors for the low QOL Index scores in their dialysis patients. 


\subsection{Key demographic and clinical predictors of QOL scores}

In the dialysis sample, the multivariate analyses for both tools showed that the long term condition and education were the only variables that were statistically significant predictors. These findings are supported by Mazes and colleagues (1997) who studied 680 adults on dialysis therapy for more than four weeks in seven dialysis centres. They used multivariate analysis to generate a model to explain the variance in the $\mathrm{QOL}$ as measured by the QOL Index scores. They found that certain co-morbidities (such as diabetes, stroke), education, age, and occupation were independently associated with QOL.

This study found that the life event and the length of time lived in the UAE variables were statistically significant in the total scores of SF-36 only. As explained earlier, major life events impact negatively on the QOL. Furthermore, the year on dialysis and knowing the cause of kidney failure variables had trends toward statistical significance in the total scores of QOL Index only. Other studies compared the relationship between the selected variables and the subscales scores of the QOL tools. In a Brazilian study by Santos and Kerr (2008) the multivariate analysis showed that time on dialysis was associated with physical function, bodily pain and vitality subscales of the SF-36 respectively. Furthermore, Neto et al. (2000) found that the number of months on haemodialysis had a significant inverse relationship with the changes in physical function, body pain, general health and vitality subscales scores of the SF-36. Mercus et al. (1997) reported a statistically significant decline in the QOL scores of the role physical, physical function, body pain and general health subscales of the SF-36 over time although no overall significant decline in the QOL scores of the vitality, social functioning, role emotional and mental health subscales of the SF-36 over time could be demonstrated. Santos and Kerr (2008) found that age was statistically significant in seven of eight domains of the SF-36, excepting bodily pain in dialysis respondents.

\subsection{Conclusion}

This study reported lower scores for the dialysis sample in all subscales of the SF-36 compared with other international studies. The total scores of the QOL Index and its subscales for the dialysis samples were higher compared to other international studies. These differences could be attributed to the tools having different subscales. The 
difference in the results from both tools reflects that the tools measure QOL differently. This difference was present when generic and disease specific tools were used.

The finding that gender, ethnicity, having full-time employment had a statistically significant positive influence on the SF-36 total scores of the QOL in both samples was supported by some of the international studies. Respondents from the community sample who were at an older age, travelled less frequently and lived longer in the UAE, had better QOL SF-36 total scores compared with respondents who were at a young age, who lived for a short period of time in the UAE and had travelled recently. The finding that having full-time employment had a statistically significant positive influence on the SF-36 total scores of the QOL in both samples was supported by some of the international studies. 


\section{Chapter 9: Conclusion}

This research was designed to investigate the QOL of a sample of dialysis patients receiving dialysis treatment at SKMC and of a community sample from Abu Dhabi, the capital of the UAE. The aims of the study were to establish what is important in respect of QOL for people living in the UAE; to identify and study the differences in the physical, psychological, social, cultural, gender and ethnicity influences on the QOL of individuals undergoing dialysis treatment and a sample from the community; to examine the impact of dialysis adequacy and nutritional status on the QOL of people on dialysis; and to compare the cultural relevancy of two QOL tools (the SF-36 and QOL Index) for dialysis patients and a sample from the community living in the UAE.

No research studies were found that addressed the QOL of dialysis patients or the community in the UAE. So, this is the first research that has examined the QOL and explored the cultural relevancy of two QOL tools for patients on dialysis and a sample from the community in UAE where the majority of the population belongs to the Muslim religion and having different ethnicities.

This research used a quantitative method through the use of the two QOL tools as well as a qualitative method through asking respondents three open-ended questions. This latter method added great value to the data collected in this research as respondents were given the opportunity to document what they considered important in relation to their QOL. This study had a high return rate, $93 \%$ in the dialysis sample and $76 \%$ in the community sample. The return rate for both samples is considered comparably higher than other studies. Moreover, the sample size of both samples is comparable with other international studies. However, the two samples were neither representative of the dialysis population nor of the general populations of UAE. The samples of both groups were taken from only one city in the UAE. There was however considerable diversity across all demographic characteristics of those who responded. The data that were collected on the blood results for the dialysis sample were only available on $84 \%$ of the sample because blood tests had not been carried out on some respondents prior to their dialysis session at the time the survey was distributed. 
This study has identified the importance of cultural relevancy in selecting QOL tools and developed a successful way of examining QOL tools for cultural relevancy. It has established that the SF-36 and the QOL Index (the two tools used in this research) were both culturally acceptable to the majority of the respondents. Cultural relevancy was ascertained by studying the QOL in the community sample, examining missing data in both samples, establishing the respondents' views on the relevancy of the tools and establishing what respondents considered was important for theirs and other UAE people's QOL.

While almost all participants answered the SF-36, some respondents did not answer a few particular questions in the QOL Index. These questions were related to sex life, relationship with partners and their chances of living as long as they would like to. The number of missing data was higher when the QOL Index was used. Questions that had a higher rate of missing data were examined for their cultural applicability and relevancy. The level of missing data is one of the indicators of the cultural relevancy. Although the tools were completed by Arabic respondents in Arabic language, translation alone is not enough. Understanding the cultural significance of certain items in tools is also important. It is important that QOL tools developed in one culture are not only tested for validity and reliability but also for cultural relevancy before using them with people from other ethnic or cultural backgrounds.

The SF-36 and QOL Index were designed to be mainly used in research examining clinical practice as opposed to being clinical tools used in everyday practice. Their use in routine clinical practice is problematic because they take a long time to complete and are difficult to score during a clinic visit. As shorter and computerised versions of tools are developed for clinical use it will be important to not only establish their reliability and validity but also their cultural relevancy. Nurses have taken the lead in promoting the concept of culturally safety and cultural safe practice. They also have the opportunity to lead the way towards having culturally relevant QOL tools as well as leading all healthcare professions in creating computerised QOL tools that are more practical to use and score as well as convenient to use in the clinical settings. 
Analysis of the open-ended questions resulted in different themes emerging. The ranking of the themes was different between the samples as each sample prioritised themes depending on their physical and emotional needs. Both samples evaluated the perception of other people differently in the community sample compared with their own evaluation about themselves. The variation in themes captured by asking the open-ended questions raise the question of the degree to which these tools capture all aspects of QOL relevant to UAE people.

The total scores of the SF-36 and QOL Index tools were both higher for the community sample compared with the dialysis sample. These scores reached statistical significance for the SF-36, but not for the QOL Index. Subscale analyses, however, showed a different pattern. The health and functioning subscale scores were statistically significantly lower in the dialysis sample compared with the community sample. Also, the family subscale scores were statistically significantly higher in the dialysis sample compared with the community sample. In the SF-36 tool, the average body pain subscale scores were lower in the community sample compared with the dialysis sample. There is no readily available explanation as to why the body pain subscale scores were low for the community and dialysis samples compared with other international studies. There is a need for further research to explore whether they were low for these particular samples or it is low for all samples in UAE.

\subsection{Significance of the study}

Healthy individuals may view or perceive QOL differently compared with patients with long term conditions such as kidney failure. Given that no studies were located examining the QOL concept among dialysis patients and people in the community in UAE, the UAE nephrology medical and nursing literature is lacking in this area of practice. Information on QOL is needed to establish how dialysis patients can be helped. This research project has generated new knowledge and expanded understanding of QOL among dialysis patients. It makes a valuable contribution to the international nursing literature with new knowledge about QOL among dialysis patients and a community sample in UAE. The information learnt about the cultural relevancy of the QOL tools will contribute to the international nursing and healthcare literature on QOL for patients with long term conditions. Studying 
QOL can benefit both dialysis patients and healthcare professionals. Dialysis patients may gain more insight about their stressors and limitations. This consequently will provide them with the opportunity to investigate their coping skills, which may help them identify poor adaptation techniques and develop healthier coping strategies. Healthcare professionals, especially nurses, spend considerable time with patients treating their chronic conditions. So, they are in a good position to take creative measures to improve the QOL of patients with long term conditions.

Studying the QOL in the UAE population helps clarify the cultural, religious and other factors which have an impact on the QOL of these samples, thereby contributing to existing knowledge. Examining the cultural relevancy of the selected tools may change how researchers and other healthcare professionals utilise those tools. The findings from this research may help nurses to determine which patients may be at risk of decreased QOL as the degree of their QOL may vary according to the severity of their illness and the degree of the disability resulting from the disease. Nurses can direct resources to areas where improvement may be required. Patients can then have a greater chance of leading a fulfilling life. Furthermore, studying the QOL should help nurses and other healthcare professionals gain knowledge and an in-depth insight of the contributing factors to better QOL among dialysis patients, so they can plan treatment by setting up specific, realistic, measurable and achievable goals. It is anticipated that the published findings of this study may contribute to improvements in care delivered to dialysis patients and will have an impact on various policy makers and might serve to stimulate discussion around QOL for patients with long term conditions.

\subsection{Limitations of the study}

This section summarises all the limitations of the study, some of which have previously been mentioned. Both samples were taken from one city in the UAE. The samples were therefore neither representative of the UAE dialysis population nor of the UAE adult population. People from different cities may think differently. People from urban areas and rural areas have different backgrounds and value systems. This study used a random group from the general population in Abu Dhabi the capital of the UAE. However, the results are not generalisable to the whole UAE population, because, the sample was taken from only this UAE city. With its limited generalisability, this study provides regional norms 
of the SF-36 and QOL Index that can be used for comparison purposes until UAE population normative values are established. Furthermore, $16 \%$ of the dialysis surveys lacked the results of blood investigations because they were not done on the month of data collection. The blood results were therefore not representative of all the respondents in the dialysis sample. Two thirds of the survey packages were completed in Arabic and one third in English. Given that the survey was anonymous no further analysis was done on the demographic characteristics of the respondents who completed the Arabic and the English versions to establish if there was a different pattern to the scores based on the versions used. Drawing a comparison between the respondents who completed the survey in Arabic and English would be useful.

Data were not examined on the types of long term conditions and the types of life events in this study; this might be considered as one of the limitation of this study. However, the focus of this research was not to study the types of long term conditions or life events for the selected samples. In contrast the focus was to study the QOL in dialysis and community samples in the UAE.

Another limitation is the impact of the reading ability of some of the respondents. For these respondents the assigned nurses translated the questions and the answers to them. The answers given may not reflect the true situation; assisting patients to complete the tools may have impacted on the validity of the results as the assistance may have influenced how respondents answered the questions. A further limitation is that no data were collected on the frequency of dialysis (two or three times a week), and the types of dialyzers used for dialysis treatment. More frequent dialysis and the uses of $\mathrm{Hi} \mathrm{Flux}$ dialyzers may have a statistically significant positively impact on the total scores of QOL for the dialysis sample.

Data collection methods are very important in capturing QOL. Other data collection methods should be used when gathering information about QOL for the first time. For example using interviews would provide participants with the opportunity to elaborate on their answers and confirm their understanding of the questions. Interviews would also means that researchers would have the chance to get more in depth knowledge and rationale from respondents about their answers and clarify other issues related to QOL. 
Despite the limitations mentioned above, this study provides good information and contributes the following to current knowledge. Firstly, testing the validity and reliability of tools is not enough. All QOL tools developed in other countries should be tested for cultural relevancy. Testing for cultural relevancy helps in identifying irrelevant or potentially offensive questions. These questions can be replaced or deleted or a non-applicable section added, thus reducing the number of missing data. Secondly, this research offers a model to establish the cultural relevancy of the tools by using four steps:

- The QOL between the dialysis patients and a sample from the community were compared.

- The cultural relevancy of each tool was studied by asking respondents directly if the tools were culturally relevant to them and what changes they would make to make them culturally relevant.

- Missing data were examined to explore whether respondents missed answering questions due to evidence of non-applicability or cultural irrelevancy.

- Open-ended questions were asked to identify what respondents personally valued in life and what contributed to this, in order to compare what they thought was important and what the QOL tools actually measure.

Thirdly, this study provides a baseline related to UAE dialysis and community QOL scores on two QOL tools. Having this baseline will enable other researchers to benchmark their findings with the findings from this study. At the time this research commenced no QOL studies were located that were undertaken in UAE or on Arab population in general. However, the recently published studies on QOL in dialysis patients from Iran and the Palestine are the starting points in building a body of literature around QOL in dialysis patients in the Middle East. This UAE study adds to this limited knowledge about QOL in dialysis patients in the Middle East and provides also an insight into QOL of a community sample. Fourthly, in this study the QOL tools used had different subscales; consequently they have captured different aspects of the QOL of respondents. Given also that the total scores were different for each tool; researchers and clinicians need to be careful when choosing QOL tools for research and clinical practice. All tools should be culturally relevant and have well established validity and reliability tests. Depending on the type of 
population, researchers are advised to select disease specific tools if they are studying the QOL of patients and to select general tools if they are studying healthy people from the community. QOL tools used in clinical settings should be disease specific, short, easy to complete and score.

Fifth, this study has shown that what people living in UAE value in life is not exactly what the tools capture and what people personally value in life could be different from other people in the same community. Six, this study has demonstrated that the presence of another long term condition in dialysis patients and the presence of long term condition with the general population is the main determinant of the QOL scores in both tools. Therefore given the focus on long term condition in nursing, and nurses being seen as the main provider of care to patients with long term condition, it is important for nurses to keep in mind the impact of long term conditions on QOL and to work creatively to improve the QOL for patients with long term conditions.

\subsection{Implications for practice and nursing care}

Renal nurses have to deal with the challenges of very high mortality, morbidity and low QOL among dialysis patients. To improve QOL, nurses need to participate actively in investigating new creative ways to improve the QOL for dialysis patients. QOL tools should be adapted to be used easily in clinical setting and tested for cultural relevancy.

The information gathered from this study highlighted certain aspects of the QOL that are important for people living in the UAE. Nurses can incorporate asking about and ultimately measuring QOL in their clinical practice. Assessing QOL in dialysis patients should give nurses more insight about patients' stressors and limitations. Moreover, it will give nurses the opportunity to investigate patients' coping skills, which may help in the early identification of poor adaptation techniques and the possibility of adapting new healthy coping strategies. Also, it gives the nurses the opportunity to gain in-depth knowledge based on what is important in maintaining and improving the QOL of dialysis patients living in the UAE.

When clinicians talk about QOL they needs to be clear about what the term QOL means to them and to their patients. They need to differentiate between health related QOL tools 
that measures health and disability and between the tools that measures patient perceptions of their QOL. Healthcare professionals have to consider mental health as well as haemoglobin levels, religious beliefs and marital status as well as parathyroid hormone, employment status and living conditions as well as dialysis efficiency, family dynamics as well as phosphorus level, nutritional status as well as food and fluid restrictions, financial status as well as iron stores. Those markers could have an effect on the $\mathrm{QOL}$ in dialysis patients. Therefore, future research should focus on looking at the $\mathrm{QOL}$ for dialysis patients from a wider perspective.

Sufficient income is an important factor in maintaining or improving the QOL in patients with long term conditions in countries that have no social security systems in place. Nursing staff should collaborate with the health care policymakers to establish regulations that ensure people with long term conditions have enough or sufficient monthly income to survive. Meeting the basic needs of these patients should take the first priority because they are not only exposed to the physical constraint of the disease but also to the psychological and social restrictions. Providing the treatment in the dialysis centres is not enough. We need to ensure that dialysis patients have the money to pay a taxi to bring them to the dialysis centres. Also, it will make no sense to the poorer dialysis patients if they are given a prescription for their medication and they do not have the money to buy it. So providing dialysis patients with full free medical insurance and sufficient monthly income might have a remarkable improvement on their QOL.

\subsection{Implications for research}

Future research should focus on getting a better matched dialysis and community sample in the UAE. The developers of the QOL Index need to consider having a non-applicable option because some of the questions do not apply to all respondents. There is a need to do more research to compare two QOL tools. Testing validity and reliability is not enough. QOL tools developed in other countries should be tested for cultural relevancy. Also, there is a need for a new QOL tool that is consistent with the culture and the religion of Muslim respondents and at the same time culturally relevant. Future research on QOL in dialysis patients from the Middle East should focus on better ways of communication with participants such as face-to-face interviews, so participants will have the chance to elaborate more on their answers and confirm their understanding of the questions and 
researcher will have the chance to get in depth knowledge by asking respondents for their rationale for their answers and clarify issues related to QOL.

More work needs to be done to culturally adapt QOL tools to make them relevant to other cultures and religions. Given that Muslim people accept their illness because it comes from Allah and consider it as a test from Allah, there is a need for more understanding of how Muslims perceive their illnesses and how they cope with their disease limitations.

There is a need to do a similar study (using both tools) on populations from other countries. For future research, the approach used to determine the cultural relevancy of the tools in this study needs to be slightly modified. Respondents need to be asked to explain their choices in open-ended questions. Given that few respondents in this research used the option of open ended questions, it may be that research addressing cultural relevancy is best done using face-to-face or telephone interviews. Future research should consider the use of the focus group approach to find out what is important and what to include in the newly developed QOL tools to make them culturally and religiously acceptable.

This study has shown that what people value in life is not exactly what the tools captured and what people valued themselves were different from other people in the same community. So, future research should pay attention to the QOL of dialysis patients from the South East Asian ethnicity. Their QOL should be investigated separately, preferably in their home countries first, and then a comparison can be made between the QOL between the dialysis patients who live in South East Asian countries and the dialysis patients from South East Asia who live in the UAE or other countries.

Research is needed on how nurses can use, interpret and incorporate the results of the QOL tools in their daily practice. It will be valuable to know how an Arab population would map their judgments and qualify their responses on metric scales compared with AfricanAmerican, Anglo-American, Hispanic and Asian survey respondents. Future research should focus on developing disease-specific computerised shorter versions tools that have open-ended questions. Finally, this is the first study that compares two QOL tools on the 
same dialysis population in UAE. Further research is needed to uncover other issues related to $\mathrm{QOL}$ in dialysis patients.

The willingness of the dialysis and the community respondents who engaged in this research has provided important new knowledge for dialysis care in UAE and for other researchers and health practitioners examining QOL. 


\section{Appendices}

\section{Appendix 1 Victoria University Ethics Committee Approval}

TE WHARE WĀNANGA O TE ŪPOKO O TE IKA A MĀUI

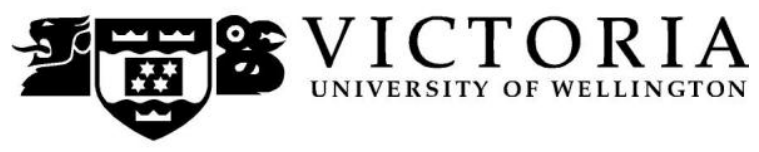

\section{MEMORANDUM}

Phone $\quad 0-4-4635676$

Fax 0-4-463 5209

\begin{tabular}{l|l}
\hline TO & Abdelbasit Ayoub \\
\hline COPY TO & Prof. Ken Walsh, Dr Kathy Nelson \\
\hline FROM & Dr Allison Kirkman, Convener, Human Ethics Committee \\
\hline
\end{tabular}

\begin{tabular}{l|l}
\hline DATE & 31 May 2007 \\
\hline PAGES & 1 \\
\hline
\end{tabular}

SUBJECT

Ethics Approval: No 66/2007, Quality of life among dialysis patients in United Arab Emirates.

Thank you for your application for ethical approval, which has now been considered by the Standing Committee of the Human Ethics Committee.

Your application has been approved and this approval continues until 30 September 2009. If your data collection is not completed by this date you should apply to the Human Ethics Committee for an extension to this approval.

Best wishes with the research.

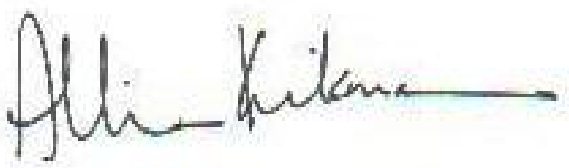

Allison Kirkman 
Appendix 2 SKMC Ethics Committee Approval

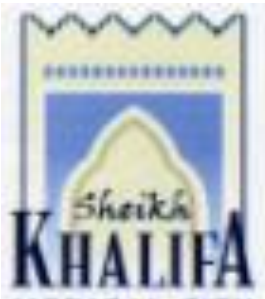

\section{RESEARCH COMMITTEE}

To : Abdelbasit Ayoub

Principal Investigator

From : Dr. Ali Khalii

Chair, Research Committeo

Re : PROTOCOL "QUALITY OF LIFE AMONG DIALYSIS PATIENTS

LIVEINUAE"

Date : $\quad 31^{\text {st }}$ May 2007

The Research Committee met on $28^{\text {th }}$ May 2007 and reviewed your research proposal. The committee is please to approve your study and you may now proceed.

We wish you success in your study and look forward to receiving an update in 12 months and the final results upon its completion.

Yours sincerely.

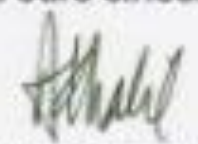

ALI KHALIL, MD, FRCPC, FACP, FACE

ox Researuh Commitee 


\section{Appendix 3: Invitation Letter for Dialysis Sample}

Research: Quality of life among dialysis patients in United Arab Emirates Invitation letter for patients to participate in the study

This letter is designed to inform you of the research project of Mr. Abdelbasit Ayoub. He is requesting that you participate in his $\mathrm{PhD}$ research which is examining the quality of life among dialysis patients who live in United Arab Emirates (UAE).

The aim of this study is to establish what is important in respect of Quality of life for people living in UAE, to identify the physical, psychological, social, and cultural influences on the quality of life of persons suffering from kidney failure and undergoing dialysis treatment.

Participation will involve completing two quality of life tools, and completing a brief questionnaire. This will take about 10-20 minutes of your time.

We do hope you are able to assist him by sharing your valuable insights and experience relevant to your quality of life as a person experiencing kidney failure and on dialysis treatment.

Respondents may choose not to participate; you can disclose any information without fear of consequences. If you choose to participate, you will be asked to sign a consent form indicating they have received information about the research project and have volunteered to participate

Thank you for your time and your generous contribution to the project.

Yours Sincerely

Abdelbasit Ayoub RN, MSN

Phone number: 0508113127

Clinical Resource Nurse for dialysis unit at SKMC 


\section{Appendix 3: Invitation Letter to Dialysis Sample (Arabic Version)}

\section{دعوة لمشاركة في بحث علمى (مرضى الكلى)}

\section{بحث عن نوعية الحياة عند مرضمى الفشل الكلوي}

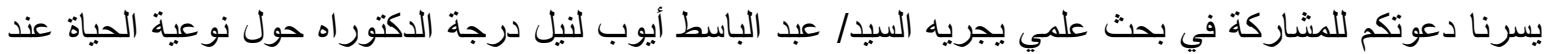

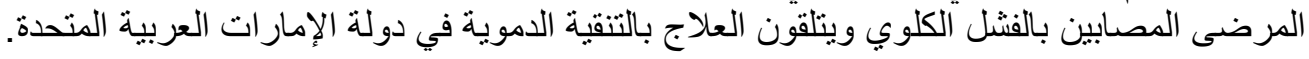

إن الهدف من هذه الدراسة هو معرفة الأشياء المهمة التي تحدد نوعية الحياة عند الأشخاص الذين يقيمون في دولة

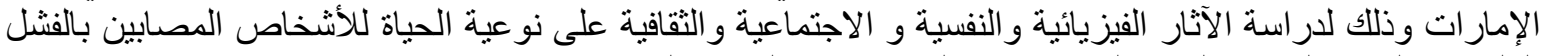
الكلوي ويتلقون العلاج بالتنقية الدموية في دولة الإلمارية ولفية الإجنات العربية المتحدة.

ولمعرفة الأشياء المهمة التي تخص مرضئ الفشل الكلوي الذين يقيمون في دولة الإمارات. سنطلب من المشاركين تعبئة استيانين عن نوعية الحياة وكذللك تعبئة استبيان آخر عن المشاركين أنفسهم. إن ذللك سيستغرق من 10 إلى 20 دقيقة من وقتك.

نأمل أن تساعدونا بالمشاركة في آرائكم وتجاربكم حول نوعية الحياة كأشخاص يعانون من الفشل الكلوي.

إن المشاركة اختيارية وللمشاركين الحق في عدم المشاركة أو المشاركة بالمعلومات دون خوف من العواتب اقب. إذا

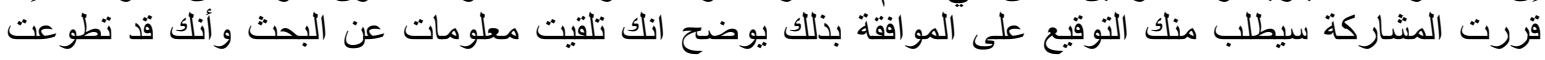
للمشاركة.

شكر ا للك على وقتلك و على مساهمتلك البناءة لإنجاح هذا المشروع.

المخلص لكم إبوبا عبد الباسط أيوب تليفون/ 050/8113127 مسؤول التعليم والتدريب في وحدة الكلى بمدينة الشيخ خليفة الطبية 


\section{Appendix 4 Dialysis Sample Consent Form}

Research: Quality of life among dialysis patients in United Arab Emirates

Patient's Information Sheet and Consent Form

\section{Principal Investigator:}

Name: Abdelbasit Ayoub

SKMC Dept.: Community Dialysis

Telephone number: 0508113127

Co - Investigator(s): I have two New Zealand based supervisors. Professor Ken Walsh and Dr. Katherine Nelson, Graduate School of Nursing, Midwifery \& Health, Victoria University of Wellington.

Emergency Telephone Number: 0508113127

\section{Background:}

I am a registered nurse and I am conducting research as part of my PhD studies about Quality of Life among dialysis patients who live in United Arab Emirates (UAE). Health care professionals help people to have a good quality of life. Little is known about what is important for people living in the United Arab Emirates in relation to Quality of Life and the research that has been done on quality of life of dialysis patients has mainly been from a Western perspective.

The study has received ethics approval from Sheikh Khalifa Medical City (SKMC) Ethics Committee and the Victoria University of Wellington, New Zealand Human Ethics Committee.

You are invited to participate in this study. This study will give you the opportunity to express your views and feelings about different dimensions of quality of life. In the light of the findings from this study health practices may change toward increasing sensitivity to the physical and psychosocial needs of dialysis patients.

\section{Purpose:}

The aim of this study is to establish what is important in respect of Quality of life for people living in UAE, to identify the physical, psychological, social, and cultural influences on the quality of life of persons undergoing dialysis.

\section{Study Procedures:}

If you choose to participate, you will be asked to complete three questionnaires including two quality of life tools and background information about you. These will take you 10-20 minutes approximately to complete. In addition, you will also give permission for the researcher to collect some clinical data from your hospital file on your kidney function and 
your nutritional status (haemoglobin level, albumin, urea, creatinine levels and urea reduction ratio)).

\section{Risks:}

No potential risk is expected form your participation in this study.

\section{Benefits:}

By participating in this study it is anticipated that the knowledge gained will be useful for clinicians to better meet the health needs important to people on dialysis in the United Arab Emirates.

\section{Reassurance:}

You have the right to refuse to participate in the study or to answer only questions you feel that you are comfortable with answering. Please be reassured that confidentiality will always be maintained no matter what choice you make. If you choose not to participate please be assured that your care will not be compromised in any way. And this is your personal choice and no body has the right to question you about it.

\section{Confidentiality:}

Any information resulting from this study will be kept strictly confidential. All documents will be identified only by code number and kept in a locked filing cabinet. Computer files will be password protected. No names will be collected on the data sheet; however a code number will be placed on the consent form and the survey tools. Findings will be presented in aggregate format. You will not be identified by name in any reports of the completed study. No one will have access to the data except me and my supervisors.

\section{Research findings}

A written summary of the findings will be put on a poster that will be placed in the dialysis unit at SKMC. The overall findings from this study will also be communicated with health care professionals involved in proving dialysis care in the UAE. Moreover, findings from the study will be presented to local and international health care providers and the academic team in Victoria University of Wellington. A copy of the thesis will be deposited in the library at Victoria University of Wellington, New Zealand and in the Sheikh Khalifa Medical City Medical Library.

\section{Contact:}

If you have any questions or desire further information with respect to this study, you should contact Mr. Abdelbasit Ayoub on Phone number 0508113127. 
If you have any concerns about your treatment or rights as a research subject, you may contact the Chair of Research Committee Dr. Patrick Killorn on phone number 026102000. Professor Ken Walsh, Clinical Professor of Nursing, Victoria University of Wellington, Wellington New Zealand. Phone number 006478398899 Ext 8175. Email address: walshk@waikatodhb.govt.nz

Dr. Katherine Nelson, Victoria University of Wellington, Wellington New Zealand, Phone number 0064 44636138. Email address: kathy.nelson@vuw.ac.nz

All participants will receive a copy of the consent form for their records.

\section{Patient Consent:}

I consent to participate in this study.

Patient Signature

Date

Recrutement nurse Signature

Date

Investigator's Signature

Date 


\section{Appendix 4 Dialysis Sample Consent Form (Arabic Version)}

\section{إذن بالمو افقة على المشاركة في بحث علمي (Dialysis Patients)}

$$
\begin{aligned}
& \text { بحث عن نوعية الحياة عند مرضى الفنشل الكلوي في دولة الإمار ات العربية المتحدة. } \\
& \text { الباحث الرئيسي: عبد الباسط أيوبة } \\
& \text { الدائرة وحدة الكلى في مدينة الثيخ خليفة البئن الطبية } \\
& \text { تلنيفون موبايل/ 050/8113127 } \\
& \text { المشرفون على البحث : المن } \\
& \text { 1. البروفسور كن والش الش (جامعة فيكتوريا في ولنغتون- نيوزيلاندا) }
\end{aligned}
$$

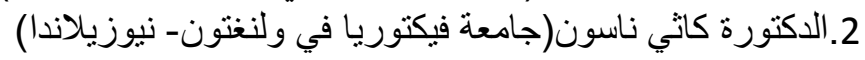

لكوستفسار: تليفون موبايل/ 050/8113127

تمهي⿻ :

أنا ممرض في مدينة الثيخ خليفة الطبية أجري بحث لنيل درجة الدكتوراه عن نوعية الحياة لدى المرضى المبل المصابين

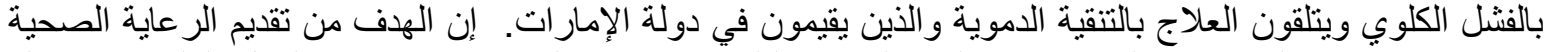

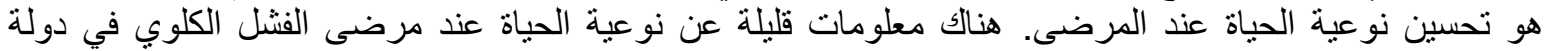

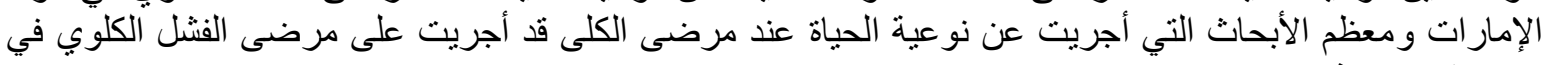

مجتمعات غربية.

هذه الدر اسة قد حصلت على مو افقة من لجنة الأبحاث في مدينة الثيخ خليفة الطبية وجامعة فيكتوريا في نيوزيلندا.

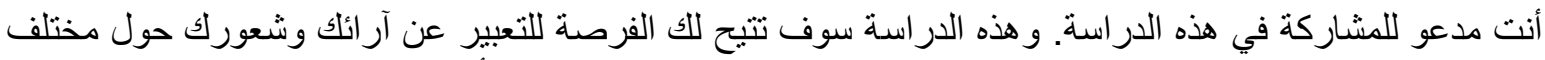

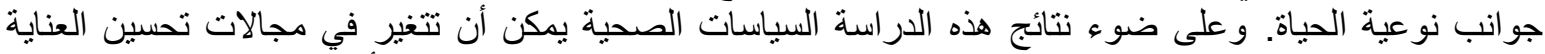

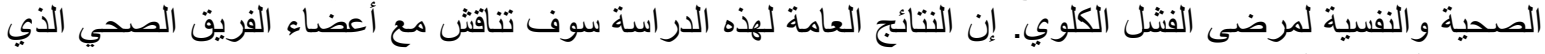
يشرف على علاجك.

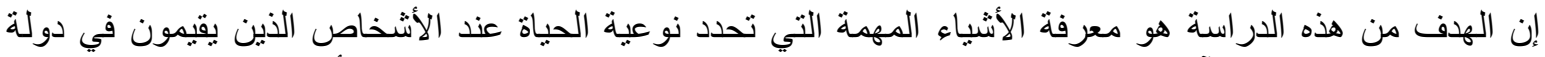

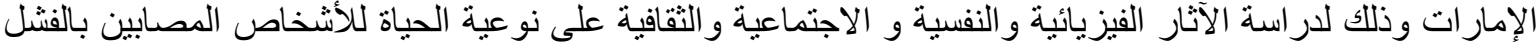

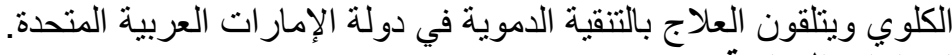

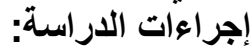

إذا رغبت بالمشاركة سوف يطلب مناسك تعبئة ثلاثة استبيانات :

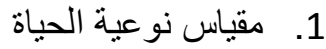

SF 36.2

3. . معلو مات مبدئية عنك

سوف يستغرق تعبئة هذه النماذج مابين 10 إلى 20 دقيقة تقريبا. كذلك سوف يتم جمع معلومات من ملفك الطبي عن كفاءة التنقية الدموية وحالة التغذية هذه النماذج.

الأخطار:

ليس هنالك أية أخطار ناتجة عن المشاركة في هذه الدراسة

المنافع: 
بمشاركتلك في هذه الدر اسة سوف تساعدنا في تقديم أفضل عناية ممكنة لتلبية حاجات مرضى الفشل الكلوي في دولة

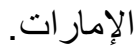

الحقوق:

للك الحق أن نرفض المشاركة في الدراسة أو أن تجيب على الأسئلة التي تشعر انك مرتاح بالإجابة عليها. نؤكد للك بأنه

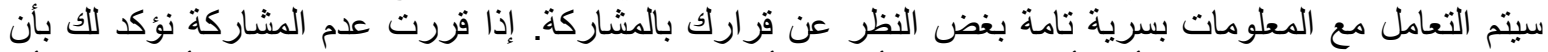

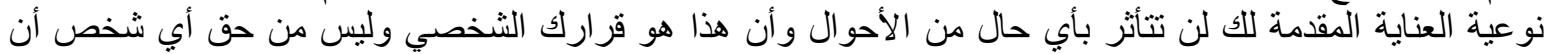
يسألك عنه.

ستعامل نتائج الدراسة بسرية نامة. وكل الوثائق ستعرف بأرقام معينة وتحفظ في خزانة انه مقفلة. وملفات الكومبيونر

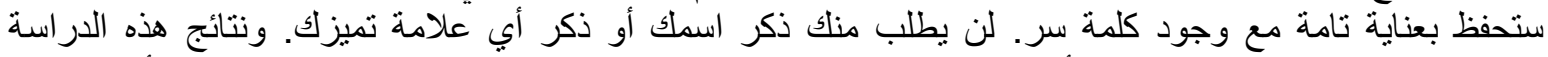

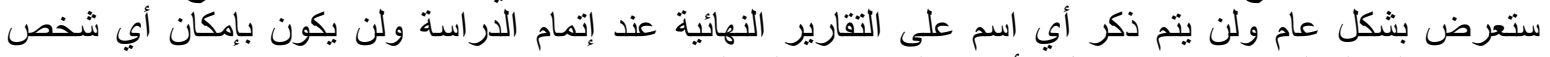
الاطلاع على المعلومات إلا من خلال الأساتذة المشرفين على الثى البحث.

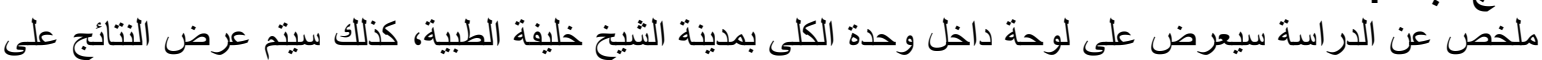

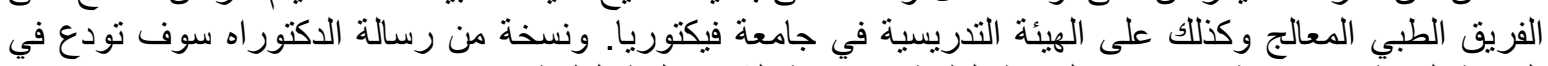
المكتبة العامة في جامعة فيكتوريا و المكتبة الطبية في مدينة الثيخ خليفة الطبية.

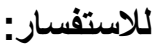

إذا كان لديك أي سؤال أو استفسار بخصوص هذه الدراسة يرجى الاتصال بالسيد/ عبد الباسط أيوب على رقم موبايل:

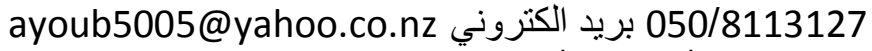
إذا كان لديك أي تعليق أو ملاحظة حول انضمامك إلى هذه الدراسة يرجى الاتصـال بالدكتور باتريك كليرون على رقم:

$$
\begin{aligned}
& \text { البروفسور كن و الش (جامعة فيكتوريا في ولنغتون- نيوزيلاندا) } \\
& \text { تليفون: } 006478398899 \text { تحويله: } 8175 \\
& \text { بريد الكتروني : walshk@waikatodhb.govt.nz }
\end{aligned}
$$

$$
\text { تلنيفون: الدكتورة كاثي ناسون(جامعة فيكتوريا في ولنغتون- نيوزيلاندا) }
$$

بريد الكتروني: Kathy.nelson@vuw.ac.nz جميع المشاركين سوف يمنحوا نسخة من الموافقة على المشاركة في هذه الدراسة

$$
\text { طاو افق المواققة: }
$$

$$
\text { توقيع المشارك } \quad \text { توقيع الممرضة }
$$




\section{Appendix 5 Survey for Dialysis sample}

\section{Quality of life among dialysis patients in United Arab Emirates}

The survey is organised into 4 parts. It takes approximately 10-20 minutes to complete.

- Part A concerns demographic and background information on quality of life and health

- Part B is the SF $\mathbf{3 6}$ quality of life tool

- Part $C$ is the Quality of Life Index Dialysis Version tool

- Part D concerns your opinion on the quality of life tools.

The survey consists of closed and open questions. If more space is required to answer any particular question please use the page at the back. All responses to the survey will be reported in group format. While quotations will be used in the reporting of survey findings, these will be presented in a non-identifiable way. Please do not sign the survey or identify yourself by name in the survey. The answers provided in this questionnaire will remain confidential. No identifiable information about you will be revealed in written or verbal reports. If there are any questions that are not applicable to you please write notapplicable. If there are any questions that you do not wish to answer, please leave blank.

\section{Part A: Background Information}

This section of the survey asks you to complete the demographic and background information on quality of life and health

1. What three things do the people living in UAE value most in life? 1

2

3

Please comment on your selection if you like

2. What three things do you personally value most in life?

1

2

3 
Please comment on your selection if you like

3. List the three things that are most important to you in maintaining or improving your quality of life?

1

2

3

Please comment on your selection if you like

4. What is your gender? Male [ ] Female [ ]

5. How long you have been on dialysis? years months

6. How old are you?

7. Do you suffer from any chronic health problems other than kidney failure such as diabetes, heart disease, asthma, cancer, rheumatoid arthritis, etc.?

Yes [ ] No [ ]

If yes, please list them

8. How long you have been living in UAE? years months

9- What do you understand was the cause of your kidney failure?

10. What is the highest level of education you have completed?

[ ] Did not attend school 
[ ] Grade 1 - 5

[ ] Grade 6 - 9

[ ] Grade 7 - 12

[ ] Secondary School Certificate

[ ] Diploma

[ ] University Degree

11. How do you describe yourself?

[ ] UAE National

[ ] Arab national

[ ] South East Asian (India, Pakistan, Bangladesh, etc)

[ ] Other (please specify):

12. What is your marital status?

$\begin{array}{lll}{[} & ] & \text { Single } \\ {[} & ] & \text { Married } \\ {[} & ] & \text { Divorced } \\ {[} & ] & \text { Widowed }\end{array}$

13. What are your current living arrangements?

[ ] Live alone

[ ] Live with husband/wife

[ ] Live with husband/wife and children

[ ] Live with parents

[ ] Live with friends

[ ] Other, please describe

14. Describe your employment status?

[ ] Working full-time

[ ] Working part-time

[ ] Unemployed

[ ] Retired

[ ] Disabled 


$$
\begin{array}{lll}
\text { [ } & \text { ] } & \text { Student } \\
\text { [ } & \text { ] } & \text { Keeping house } \\
\text { [ } & \text { ] } & \text { Other, please describe }
\end{array}
$$

15. When was the last time you travelled out of the country?

years months

16. Have you had any major life events in the last 12 months in addition to your kidney disease such as: marriage, accident, death of a family member, etc..?

[ ] Yes [ ] No

If yes, please specify

17. What is your religion?

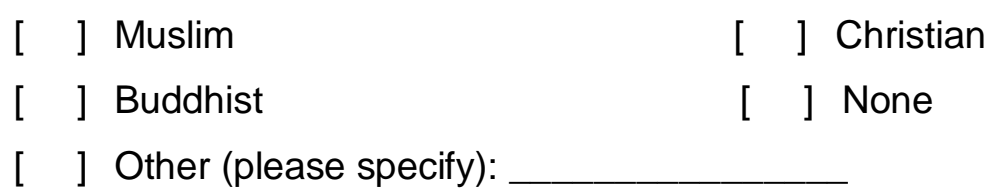

Thank you for completing this background information. 


\section{Part B: SF 36 quality of life tool (Dialysis patients)}

This section of the survey asks you to complete the SF 36 quality of life tool

\section{SF-36 HEALTH SURVEY}

INSTRUCTIONS: This survey asks for your views about your health. This information will help keep track of how you feel and how well you are able to do your usual activities.

Answer every question by marking the answer as indicated. If you are unsure about how to answer a question, please give the best answer you can.

1. In general, would you say your health is:

$$
\text { (circle one) }
$$

Excellent

2. Compared to one year ago, how would you rate your health in general now?

$$
\text { (circle one) }
$$

Much better now than one year ago ................................1

Somewhat better now than one year ago ........................... 2

About the same as one year ago ...................................... 3

Somewhat worse now than one year ago ..........................4

Much worse now than one year ago ................................. 5 
3. The following questions are about activities you might do during a typical day. Does your health now limit you in these activities? If so, how much?

\begin{tabular}{|c|c|c|c|}
\hline ACTIVITIES & $\begin{array}{c}\text { Yes, } \\
\text { Limited } \\
\text { A Lot }\end{array}$ & $\begin{array}{c}\text { Yes, } \\
\text { Limited } \\
\text { A Little }\end{array}$ & $\begin{array}{c}\text { No, Not } \\
\text { Limited } \\
\text { At All }\end{array}$ \\
\hline $\begin{array}{l}\text { a. Vigorous activities, such as running, lifting heavy objects, } \\
\text { participating in strenuous sports }\end{array}$ & 1 & 2 & 3 \\
\hline $\begin{array}{l}\text { b. Moderate activities, such as moving a table, pushing a } \\
\text { vacuum cleaner, bowling, or playing golf }\end{array}$ & 1 & 2 & 3 \\
\hline c. Lifting or carrying groceries & 1 & 2 & 3 \\
\hline d. Climbing several flights of stairs & 1 & 2 & 3 \\
\hline e. Climbing one flight of stairs & 1 & 2 & 3 \\
\hline f. Bending, kneeling, or stooping & 1 & 2 & 3 \\
\hline g. Walking more than a mile & 1 & 2 & 3 \\
\hline h. Walking half a mile & 1 & 2 & 3 \\
\hline i. Walking one hundred yards & 1 & 2 & 3 \\
\hline j. $\quad$ Bathing or dressing yourself & 1 & 2 & 3 \\
\hline
\end{tabular}

4. During the past 4 weeks, have you had any of the following problems with your work or other regular daily activities as a result of your physical health?

(circle one number on each line)

\begin{tabular}{|c|c|c|}
\hline & YES & NO \\
\hline a. $\begin{array}{l}\text { Cut down on the amount of time you spent on work or other } \\
\text { activities }\end{array}$ & 1 & 2 \\
\hline b. Accomplished less than you would like & 1 & 2 \\
\hline c. Were limited in the kind of work or other activities & 1 & 2 \\
\hline d. Had difficulty performing the work or other activities (for \\
example, it took extra effort)
\end{tabular}


5. During the past 4 weeks, have you had any of the following problems with your work or other regular daily activities as a result of any emotional problems (such as feeling depressed or anxious)?

(circle one number on each line)

\begin{tabular}{|l|c|c|}
\hline & YES & NO \\
\hline a. Cut down on the amount of time you spent on work or other activities & 1 & 2 \\
\hline b. Accomplished less than you would like & 1 & 2 \\
\hline c. Didn't do work or other activities as carefully as usual & 1 & 2 \\
\hline
\end{tabular}

6. During the past 4 weeks, to what extent has your physical health or emotional problems interfered with your normal social activities with family, friends, neighbours, or groups?

(circle one)

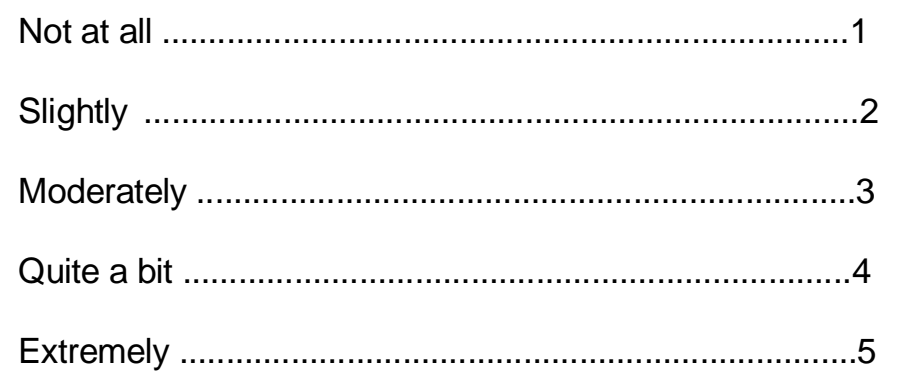

7. How much bodily pain have you had during the past 4 weeks?

(circle one)

Very mild


8. During the past 4 weeks, how much did pain interfere with your normal work (including both work outside the home and housework)?

(circle one)

Not at all 1

A little bit .2

Moderately .3

Quite a bit .4

Extremely .5

9. These questions are about how you feel and how things have been with you during the past 4 weeks. For each question, please give the one answer that comes closest to the way you have been feeling. How much of the time during the past 4 weeks -

\begin{tabular}{|c|c|c|c|c|c|c|}
\hline & $\begin{array}{l}\text { All of } \\
\text { the } \\
\text { Time }\end{array}$ & $\begin{array}{l}\text { Most of } \\
\text { the } \\
\text { Time }\end{array}$ & $\begin{array}{l}\text { A Good } \\
\text { Bit of } \\
\text { the Time }\end{array}$ & $\begin{array}{l}\text { Some of } \\
\text { the Time }\end{array}$ & $\begin{array}{l}\text { A Little } \\
\text { of the } \\
\text { Time } \\
\end{array}$ & $\begin{array}{l}\text { None } \\
\text { of the } \\
\text { Time }\end{array}$ \\
\hline a. Did you feel full of life? & 1 & 2 & 3 & 4 & 5 & 6 \\
\hline $\begin{array}{l}\text { b. Have you been a very nervous } \\
\text { person? }\end{array}$ & 1 & 2 & 3 & 4 & 5 & 6 \\
\hline $\begin{array}{l}\text { c. Have you felt so down in the } \\
\text { dumps that nothing could cheer } \\
\text { you up? }\end{array}$ & 1 & 2 & 3 & 4 & 5 & 6 \\
\hline $\begin{array}{l}\text { d. Have you felt calm and } \\
\text { peaceful? }\end{array}$ & 1 & 2 & 3 & 4 & 5 & 6 \\
\hline e. Did you have a lot of energy? & 1 & 2 & 3 & 4 & 5 & 6 \\
\hline $\begin{array}{ll}\text { f. Have you felt downhearted and } \\
\text { low? }\end{array}$ & 1 & 2 & 3 & 4 & 5 & 6 \\
\hline g. Did you feel worn out? & 1 & 2 & 3 & 4 & 5 & 6 \\
\hline $\begin{array}{l}\text { h. Have you been a happy } \\
\text { person? }\end{array}$ & 1 & 2 & 3 & 4 & 5 & 6 \\
\hline i. $\quad$ Did you feel tired? & 1 & 2 & 3 & 4 & 5 & 6 \\
\hline
\end{tabular}


10. During the past 4 weeks, how much of the time has your physical health or emotional problems interfered with your social activities (like visiting with friends, relatives, etc.)?

(circle one)

All of the time …..............................................................

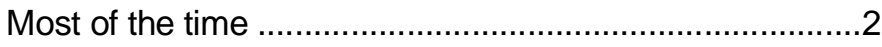

Some of the time ….......................................................

A little of the time …......................................................

None of the time ……................................................

11. How TRUE or FALSE is each of the following statements for you?

\begin{tabular}{|c|c|c|c|c|c|}
\hline & $\begin{array}{l}\text { Definitely } \\
\text { True }\end{array}$ & $\begin{array}{l}\text { Mostly } \\
\text { True }\end{array}$ & $\begin{array}{l}\text { Don't } \\
\text { Know }\end{array}$ & $\begin{array}{l}\text { nostly } \\
\text { False }\end{array}$ & $\begin{array}{c}\text { Defin line) } \\
\text { Definitely } \\
\text { False }\end{array}$ \\
\hline $\begin{array}{l}\text { a. I seem to get ill more easily than } \\
\text { other people }\end{array}$ & 1 & 2 & 3 & 4 & 5 \\
\hline b. I am as healthy as anybody I know & 1 & 2 & 3 & 4 & 5 \\
\hline c. I expect my health to get worse & 1 & 2 & 3 & 4 & 5 \\
\hline d. My health is excellent & 1 & 2 & 3 & 4 & 5 \\
\hline
\end{tabular}




\section{Questions Concerns your opinions on the SF-36}

1-Do you feel that this tool is relevant to your culture?

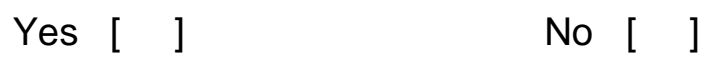

2. What, if any question / questions should be added to the tool to make it more culturally relevant for people in the United Arab Emirates?

3. What, if any question / questions should be deleted to the tool to make it more culturally relevant for people in the United Arab Emirates?

4. How good is this tool in capturing the quality of life for people on dialysis?

\section{Part C: Quality of Life Index tool}

This section of the survey asks you to complete the Quality of Life Index tool 


\section{Ferrans and Powers \\ QUALITY OF LIFE INDEX ${ }^{c}$ \\ DIALYSIS VERSION - III}

DARI 1. For cach of the following plesse choose the anower that best describes how satisfied you are with that area of your life. Please mark your answer by circling the number. There are no right or wrong answers.

HOW S.ATISFIED ARE YOU WITH:

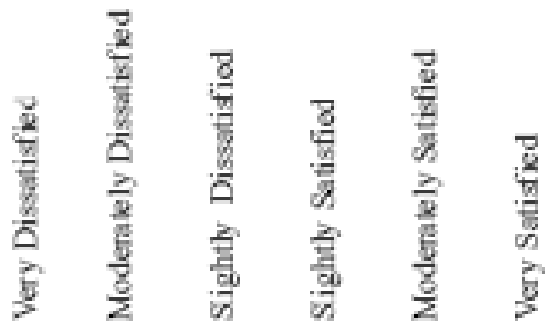

\begin{tabular}{|c|c|c|c|c|c|c|}
\hline 1. Your bealth? & 1 & 2 & 3 & 4 & 5 & 6 \\
\hline 2. Your health care? & 1 & 2 & 3 & 4 & 5 & 6 \\
\hline 3. The amount of energy you bave for everyday activities? & 1 & 2 & 3 & 4 & 5 & 6 \\
\hline 4. Your ability to take care of yourself without help? & 1 & 2 & 3 & 4 & 5 & 6 \\
\hline 5. The likelihood you will get a kidney transplant? & 1 & 2 & 3 & 4 & 5 & 6 \\
\hline \multicolumn{7}{|l|}{ 6. The changes you have had to make in your life because } \\
\hline of kidney failure (such as diet and need for dialysis)? & 1 & 2 & 3 & 4 & 5 & 6 \\
\hline 7. The amount of control you have over your life? & 1 & 2 & 3 & 4 & 5 & 6 \\
\hline 8. Your chances of living as long as you would like? & 1 & 2 & 3 & 4 & 5 & 6 \\
\hline 9. Your family's bealth? & 1 & 2 & 3 & 4 & 5 & 6 \\
\hline 10. Your children? & 1 & 2 & 3 & 4 & 5 & 6 \\
\hline 11. You faudly's lappiness? & 1 & 2 & 3 & 4 & 5 & $\sigma$ \\
\hline 12. Your sex life? & 1 & 2 & 3 & 4 & 5 & 6 \\
\hline 13. Your spouse, lover, or partmer? & 1 & 2 & 3 & 4 & 5 & 6 \\
\hline 11. Your fricnds? & 1 & 2 & 3 & 1 & 5 & 6 \\
\hline 15. The emotional support you get from your family? & 1 & 2 & 3 & 4 & 5 & 6 \\
\hline
\end{tabular}

(Please Go To Next Page)

o Copyright 1984 \& 1998 Carol Estwing Fenaus and Marjorie J. Powers (Do not we without parmission). 
HOW S.ATISFIED ARE YOU WITH:

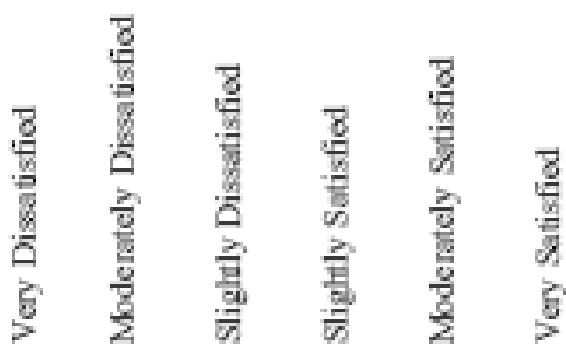

16. The emotional support you get from people other

\begin{tabular}{lllllll}
\hline than your family? & 1 & 2 & 3 & 4 & 5 & 6 \\
\hline Your ability to take care of family responsibilities? & 1 & 2 & 3 & 4 & 5 & 6 \\
\hline . How useful you are to others? & 1 & 2 & 3 & 4 & 5 & 6 \\
\hline The amount of worries in your life? & 1 & 2 & 3 & 4 & 5 & 6 \\
\hline Your neighborhood? & 1 & 2 & 3 & 4 & 5 & 6 \\
\hline Your houne, apartment, or place where you live? & 1 & 2 & 3 & 4 & 5 & 6 \\
\hline Your job (if enuployed)? & 1 & 2 & 3 & 4 & 5 & 6 \\
\hline Not baving a job (if unemployed, retired, or disabled)? & 1 & 2 & 3 & 4 & 5 & 6 \\
\hline 4our education? & 1 & 2 & 3 & 4 & 5 & 6 \\
\hline How well you can take care of your financial needs? & 1 & 2 & 3 & 4 & 5 & 6 \\
\hline The things you do for fun? & 1 & 2 & 3 & 4 & 5 & 6 \\
\hline Your chances for a bappy future? & 1 & 2 & 3 & 4 & 5 & 6 \\
\hline Your peace of mind? & 1 & 2 & 3 & 4 & 5 & 6 \\
\hline Your faith in God? & 1 & 2 & 3 & 4 & 5 & 6 \\
\hline . Your achievement of personal goals? & 1 & 2 & 3 & 4 & 5 & 6 \\
\hline . Your bappiness in general? & 1 & 2 & 3 & 4 & 5 & 6 \\
\hline Your life in general? & 1 & 2 & 3 & 4 & 5 & 6 \\
\hline Your personal appearance? & 1 & 2 & 3 & 4 & 5 & 6 \\
\hline 4. Yourself in general? & 2 & 3 & 4 & 5 & 6 \\
\hline
\end{tabular}

(Please Go To Next Page)

o Copyright 1984 \& 1998 Carol Estwing Farrans and Majons J. Powors (Do not use withont permission). 
PART 2. For each of the following, please choose the answer that best describes how important that area of your life is to you. Please mark your answer by circling the number. There are no right or wrong answers.

HOW IMPORTANT TO YOU IS:

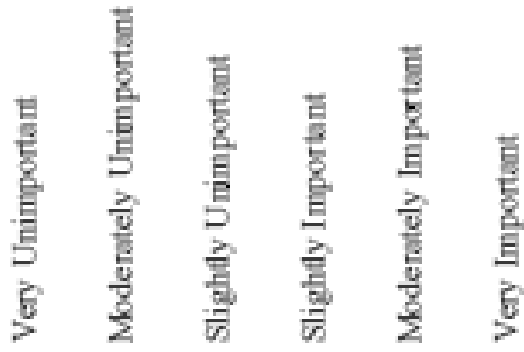

\begin{tabular}{lllllll}
\hline 1. Your bealth? & 1 & 2 & 3 & 4 & 5 & 6 \\
\hline 2. Your bealth care? & 1 & 2 & 3 & 4 & 5 & 6 \\
\hline 3. Having enough energy for everyday activities? & 1 & 2 & 3 & 4 & 5 & 6 \\
\hline 4. Taking care of yourself without help? & 1 & 2 & 3 & 4 & 5 & 6 \\
\hline 5. Getting a kidney transplant? & 1 & 2 & 3 & 4 & 5 & 6 \\
\hline $\begin{array}{l}\text { 6. The changes you have had to make in your life because of } \\
\text { kidney failure (such as diet and need for dialysis)? }\end{array}$ & 1 & 2 & 3 & 4 & 5 & 6 \\
\hline 7. Having coutrol over your life? & 1 & 2 & 3 & 4 & 5 & 6 \\
\hline $\begin{array}{l}\text { 8. Living as long as you would like? } \\
\text { 9. Your family's health? }\end{array}$ & 1 & 2 & 3 & 4 & 5 & 6 \\
\hline $\begin{array}{l}\text { 10. Your children? } \\
\text { 11. Your family's happiness? }\end{array}$ & 1 & 2 & 3 & 4 & 5 & 6 \\
\hline 12. Your sex life? & 1 & 2 & 3 & 4 & 5 & 6 \\
\hline $\begin{array}{l}\text { 13. Your spouse, lover, or partner? } \\
\text { 14. Your friends? }\end{array}$ & 1 & 2 & 3 & 4 & 5 & 6 \\
\hline $\begin{array}{l}\text { 15. The emotional support you get from your family? } \\
\text { 16. The emotional support you get from people other } \\
\text { than your family? }\end{array}$ & 1 & 2 & 3 & 4 & 5 & 6 \\
\hline
\end{tabular}

(Please Go To Next Page)

o Copyright 1984 \& 1998 Casol Estwieg Farrans and Marjorio J. Porrors (Do not use withont persaisnion). 
HOW IMPORTANT TO YOU IS:

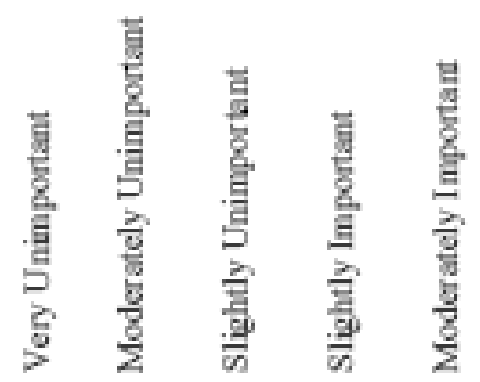

\begin{tabular}{lllllll}
\hline 17. Taking care of family responsibilities? & 1 & 2 & 3 & 4 & 5 & 6 \\
\hline 18. Being useful to others? & 1 & 2 & 3 & 4 & 5 & 6 \\
\hline 19. Having no worries? & 1 & 2 & 3 & 4 & 5 & 6 \\
\hline 20. Your neighborhood? & 1 & 2 & 3 & 4 & 5 & 6 \\
\hline 21. Your houne, apartment, or place where you live? & 1 & 2 & 3 & 4 & 5 & 6 \\
\hline 22. Your job (if enuployed)? & 1 & 2 & 3 & 4 & 5 & 6 \\
\hline 23. Having a job (if unemployed, retired, or disabled)? & 1 & 2 & 3 & 4 & 5 & 6 \\
\hline 24. Your education? & 1 & 2 & 3 & 4 & 5 & 6 \\
\hline 25. Being able to take care of your financial needs? & 1 & 2 & 3 & 4 & 5 & 6 \\
\hline 26. Doing things for fun? & 1 & 2 & 3 & 4 & 5 & 6 \\
\hline 27. Having a bappy future? & 1 & 2 & 3 & 4 & 5 & 6 \\
\hline 28. Peace of mind? & 1 & 2 & 3 & 4 & 5 & 6 \\
\hline 29. Your faith in God? & 1 & 2 & 3 & 4 & 5 & 6 \\
\hline 30. Achieving your personal goals? & 1 & 2 & 3 & 4 & 5 & 6 \\
\hline 31. Your happiness in general? & 1 & 2 & 3 & 4 & 5 & 6 \\
\hline 32. Being satisfied with life? & 1 & 2 & 3 & 4 & 5 & 6 \\
\hline 33. Your personal appearance? & 1 & 2 & 3 & 4 & 5 & 6 \\
\hline 34. Are you to yourself? & & 2 & 3 & 4 & 5 & 6 \\
\hline
\end{tabular}

O Copyright 1984 \& 1998 Carol Estwieg Farrms and Marjoris J. Powars (Do not use withont permisnion). 


\section{Questions Concerns your opinions on the Quality of Life Index tool}

1. Do you feel that this tool is relevant to your culture?

$$
\text { Yes [ ] No [ ] }
$$

2. What, if any question / questions should be added to the tool to make it more culturally relevant for people in the United Arab Emirates?

3. What, if any question / questions should be deleted to the tool to make it more culturally relevant for people in the United Arab Emirates?

4. How good is this tool in capturing the quality of life for people on dialysis? 


\section{Part D: your opinions on the SF 36 and Quality of Life Index.}

This section of the survey asks you to answer one question about whether you prefer the SF-36 or the Quality of Life Index tool.

\section{Comparing the two tools}

Which is tool is more culturally relevant for measuring your QOL?

[ ] Neither tool is culturally relevant

[ ] SF 36 more culturally relevant

[ ] Quality of life Index more culturally relevant

[ ] Both tools are culturally relevant

Thank you for completing this survey.

Please place the completed survey to in the box or return it to Mr. Abdelbasit Ayoub or mail it to Abdelbasit Ayoub, Dialysis Unit, SKMC, P.O. Box: 51900 Abu Dhabi UAE Please mail back your reply by -/-/ 2007

\section{Blood results value}

$\mathrm{Hb}$ :

Serum albumin:

Pre-dialysis BUN (g/L):

Pre-dialysis creatinine $(\mathrm{g} / \mathrm{L})$ :

Urea Reduction ratio: 


\section{Research: Quality of life among dialysis patients in United Arab Emirates}

\section{Invitation letter for volunteers to participate in the study}

This letter is designed to inform you of the research project of Mr. Abdelbasit Ayoub. He is requesting that you participate in his $\mathrm{PhD}$ research which is examining the quality of life among dialysis patients who live in United Arab Emirates (UAE).

The aim of this study is to establish what is important in respect of Quality of Life for people living in UAE, to identify the physical, psychological, social, and cultural influences on the quality of life of persons suffering from kidney failure and undergoing dialysis treatment.

To establish what is important for people living in UAE a considerable number of healthy people from different age groups are required to participate in the study. Participation will involve completing two Quality of Life tools, and completing a brief questionnaire. This will take about 10-20 minutes of your time.

We do hope you are able to assist him by sharing your valuable insights and experience relevant to your quality of life as a normal healthy individual.

Participants may choose not to participate; you can disclose any information without fear of consequences. If you choose to participate, you will be asked to sign a consent form indicating you have received information about the research project and have volunteered to participate.

Thank you for your time and your generous contribution to the project.

Yours Sincerely

Abdelbasit Ayoub RN, MSN

Phone Number: 0508113127

Clinical Resource Nurse for dialysis unit at SKMC 


\section{Appendix 6 Invitation Letter for community Sample (Arabic Version) \\ دعوة لمشاركة في بحث علمى ( أشخاص سليمين)}

\section{بحث عن نوعية الحياة عند مرضى الفشل الكلوي}

يسرنا دعوتكم للمشاركة في بحث علمي يجريه السيد/ عبد الباسط أيوب لنيل درجة الدكتوراه حول نوعية الحياة عند

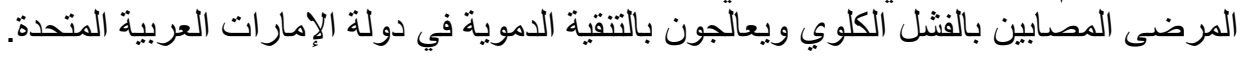

إن الهدف من هذه الدراسة هو معرفة الأشياء المهمة التي تحدد نوعية الحياة عند الأشخاص الذين يقيمون في دولة الإئة

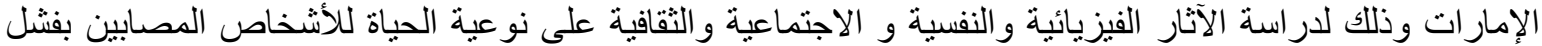
كلوي ويتلقون العلاج بالتنقية الدموية في دولة الإنية الإمار ات العربة العربية المتحدة.

ولمعرفة الأشياء المهمة التي تخص مرضى الفشل الكلوي الذين يقيمون في دولة الإمارات. سوف يتم دعوة عدد معين

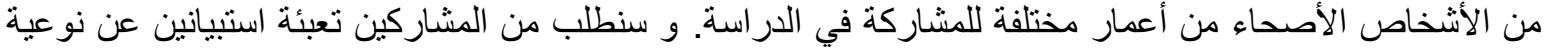

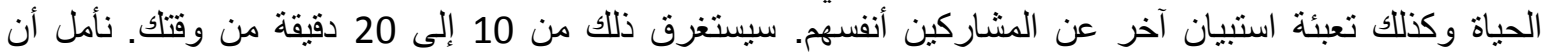
تساعدونا بالمشاركة في آر ائكم وتجاربكم حول نوعية الحياة كأشخاص سليمين من الناحية الصحية.

إن المشاركة اختيارية وللمشاركين الحق في عدم المشاركة أو المشاركة بالمعلومات دون خوف من العواقب. إذا

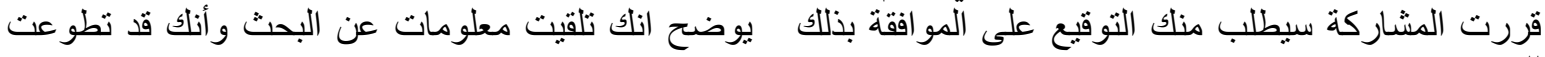
لإنشاركة.

شكر ا للك على وقتلك و على مساهمتلك البناءة لإنجاح هذا المشروع.

المخلص لكم عبد الباسط أيوب تليفون/ 050/8113127 مسؤول التعليم والتدريب في وحدة الكلى بمدينة الثيخ خليفة الطبية 


\section{Appendix 7 Community Sample Consent Form Consent Form}

\section{Research: Quality of life among dialysis patients in United Arab Emirates}

\section{Volunteer's Information Sheet and Consent Form \\ Principal Investigator:}

Name: Abdelbasit Ayoub

SKMC Dept: Community Dialysis

Telephone number: 0508113127

Co - Investigator(s): I have two New Zealand based supervisors. Professor Ken Walsh and Dr Katherine Nelson, Graduate School of Nursing, Midwifery \& Health, Victoria University of Wellington.

\section{Emergency Telephone Number: 0508113127}

\section{Background:}

I am a registered nurse and I am conducting research as part of my $\mathrm{PhD}$ studies about quality of life among dialysis patients who live in United Arab Emirates (UAE). Health care professionals help people to have a good quality of life. Little is know about Quality of Life of people living in the United Arab Emirates, and the research that has been done on quality of life of dialysis patients has mainly been from a western perspective. The study has received ethics approval from Sheikh Khalifa Medical City (SKMC) ethics committee and the Victoria University of Wellington, New Zealand Human Ethics Committee.

You are invited to participate in this study. Your participation will help me in comparing what is important in quality of life between general population and persons on dialysis from SKMC. The findings from this study will be used to inform the services provided to people who are on dialysis.

\section{Purpose:}

The aim of this study is to establish what is important in respect of quality of life for people living in UAE, to identify the physical, psychological, social, and cultural influences on the quality of life of persons undergoing dialysis. 


\section{Study Procedures:}

If you choose to participate, you will be asked to complete three questionnaires, a Quality of Life tool, SF 36 tool and a questionnaire about you. These will take you 10-20 minutes approximately to complete.

\section{Risks:}

No potential risk is expected from your participation in this study.

\section{Benefits:}

By participating in this study you will give us the information needed for us to help determine whether a tool used internationally to examine quality of life is relevant to people who live in the United Arab Emirates. The data will also be used to establish whether healthy people from UAE and people on dialysis in UAE value different things in relation to Quality of Life. The research findings will be used to improve the health care of dialysis patients

\section{Reassurance:}

Participation in this is study is your personal choice. If you choose not to participate, I will respect your decision and no body has the right to question you about it. Consent is indicated by completing the questionnaire

\section{Confidentiality:}

Any information resulting from this study will be kept strictly confidential. All documents will be identified only by code number and kept in a locked filing cabinet. Computer files will be password protected. No names will be collected on the data sheet. You will not be identified by name in any reports of the completed study.

You have the right to refuse to participate in the study or to answer only questions you feel that you are comfortable with. Please be reassured that confidentiality and anonymity will be always maintained and that participation is voluntary. No one will have access to the data except me and my supervisors.

\section{Research findings}

A written summary of the findings will be put on a poster that will be placed in the dialysis unit at SKMC. Moreover, findings from the study will be presented to local and international health care providers and the academic team in Victoria University of Wellington. A copy of the thesis will be deposited in the library at Victoria University of Wellington, New Zealand and in the Sheikh Khalifa Medical City Medical Library.

\section{Contact:}


If you have any questions or desire further information with respect to this study, you should contact Mr. Abdelbasit Ayoub on Phone number 0508113127

If you have any concerns about your treatment or rights as a research subject, you may contact Chair of Research Committee Dr. Patrick Killorn on phone number 026102000

Professor Ken Walsh, Clinical Professor of Nursing, Victoria University of Wellington, Wellington New Zealand. Phone number 006478398899 Ext 8175. Email address: walshk@waikatodhb.govt.nz

Dr. Katherine Nelson, Victoria University of Wellington, Wellington New Zealand, Phone number 0064 44636138. Email address: kathy.nelson@vuw.ac.nz

All participants will receive a copy of the consent form for their records.

\section{Consent:}

I consent to participate in this study.

Patient Signature

Date

Investigator Signature

Date 


\title{
Appendix 7 Community Sample Consent Form (Arabic Version)
}

\section{طeneral Population - طلب المواققة على المشاركة في بحث علمي}

\author{
بحث عن نوعية الحياة عند مرضى الفثل الكلوي في دولة الإمارات العربية المتحدة. \\ الباحث الرئيسي: عبد الباسط أيوب. \\ الدائرة وحدة الكلى في مدينة الثيخ خليفة الطبية النيانية \\ تليفون موبايل/ 050/8113127

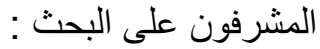 \\ 1. البروفسور كن و الش (جامعة فيكتوريا في ولنغتون- نيوزيلاندا) \\ 2.الدكتورة كاثي ناسون(جامعة فيكتوريا في ولنغتون- نيوزيلانياندا)
}

للاستفسار ات: تليفون موبايل/ 050/8113127

تمهيد :

أنا ممرض في مدينة الثيخ خليفة الطبية أجري بحثا لنيل درجة الدكتوراه عن نوعية الحياة عند المرضى المبل المصابين

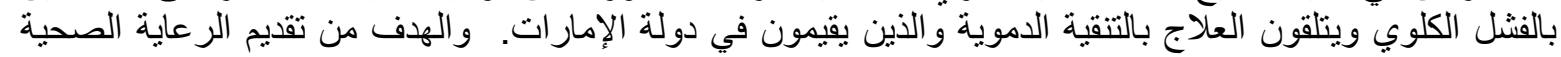

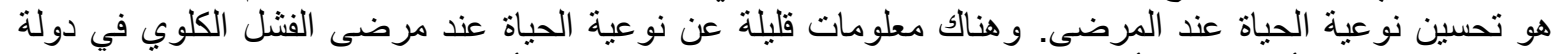

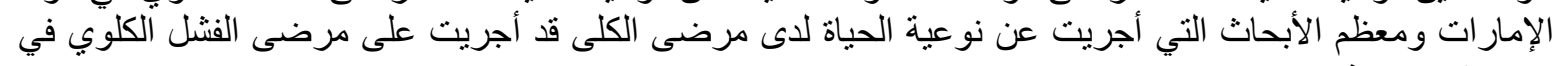

هذه الدر اسة قد حصلت على مو افقة من لجنة الأبحاث في مدينة الثيخ خليفة الطبية وجامعة فيكتوريا في نيوزيلندا.

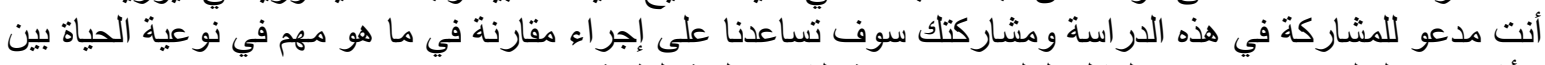

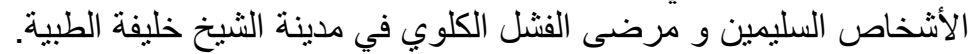
إن نتائج هذه الدراسة ستساعد الفريق الطبي الذي يعالج مرضى الفي الفشل الكلوي بواسطة التنقية الدموية.

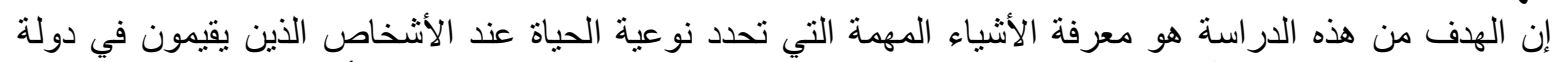

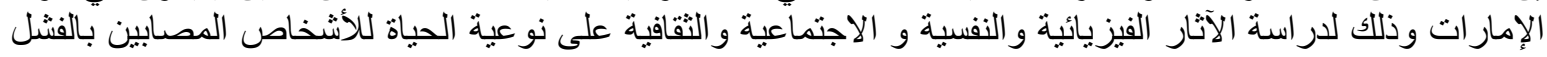

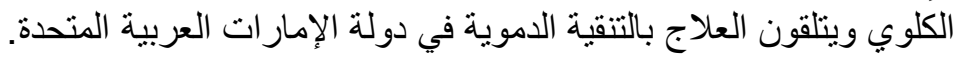

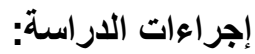

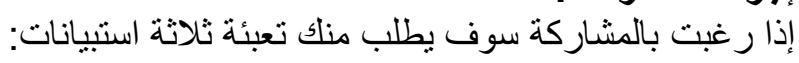

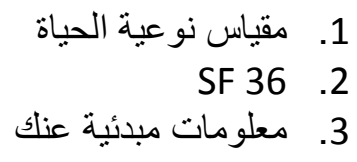

سوف يستغرق تعبئة هذه النماذج ما بين 10إلى 20 دقيقة تقريبا. الأخطار: ليس هنالك أية أخطار ناتجة عن المشاركة في هذه الدراسة 


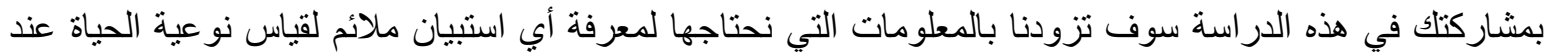

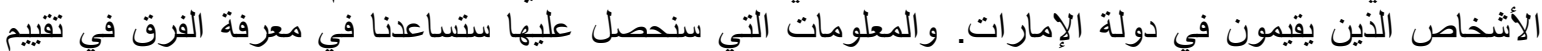

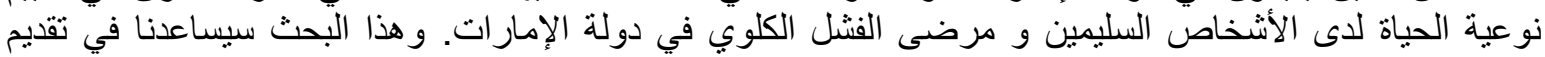

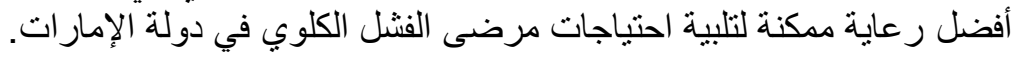

للك الحق أن ترفض المشاركة في الدراسة أو أن تجيب على الأسئلة التي تشعر بأنك مرتاح بالإجابة عليها. نؤكد للك

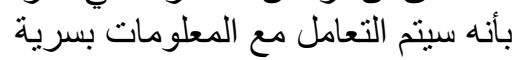

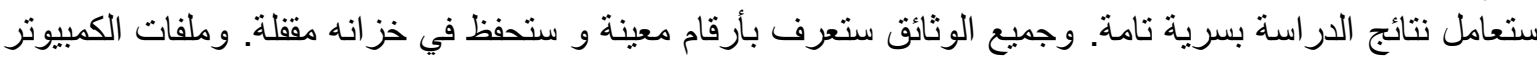

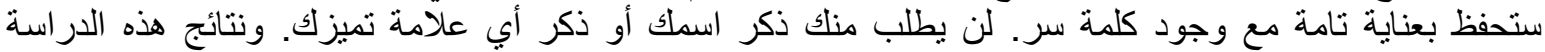

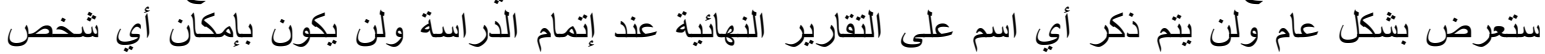
الإطلاع على المعلومات إلا من خلال الأساتذة المشرفين على البحث. نتائج البحث:

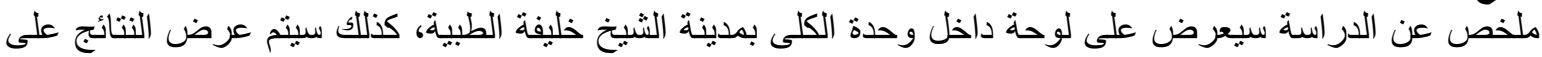

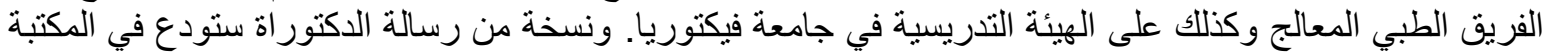
العامة في جامعة فيكتوريا والمكتبة الطبية في مدينة الثنيخ خليفة الطبية.

ل اللاستفسار:

إذا كان لديك أي سؤال أو توضيح بخصوص هذه الدراسة يرجى الاتصال بالسيد/ عبد الباسط أيوب على رقم موبايل :

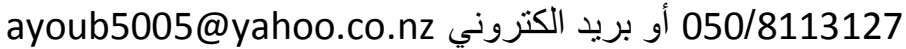

إذا كان لديك أي تعليق أو ملاحظة حول انضمامك إلى هذه الدراسة يرجى الاتصال بالدكتور باتريك كليرون على رقم:

البروفسور كن و الش (جامعة فيكتوريا في ولنغتون- نيوزيلندا)

تلليفون: 006478398899 تحويله: 8175

walshk@waikatodhb.govt.nz : بريد الكتروني

الدكتورة كاثي ناسون(جامعة فيكتوريا في ولنغتون- نيوزيلندا)

تليفون: 006444636138

بريد الكتروني: Kathy.nelson@vuw.ac.nz

جميع المشاركين سوف يمنحون نسخة من الموافقة على المشاركة في هذه الدراسة

إذإن الموافقة:

أوافق على المشاركة في الدراسة

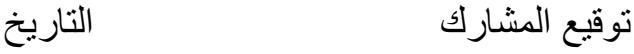

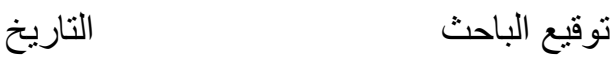




\section{Appendix 8 Survey for Community sample}

\section{Appendix 6 Survey for Community sample (Arabic version)}
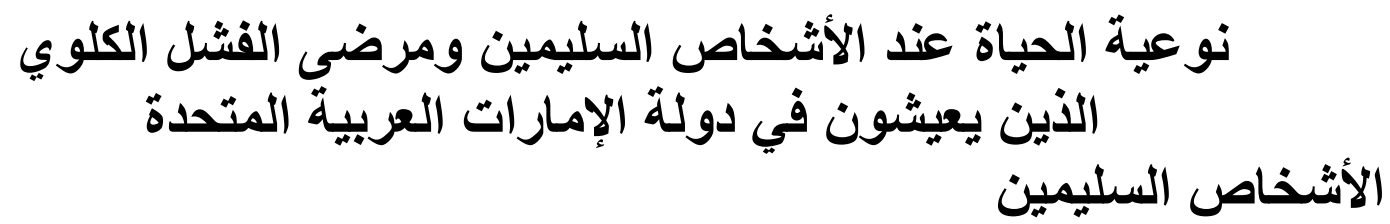

$$
\begin{aligned}
& \text { ينقسم هذا الإستبيان الى أربعة أجز اء. وسيستغرق 10-20 دقيقة من وقتاك. }
\end{aligned}
$$

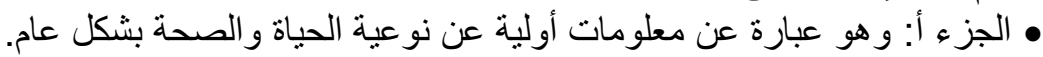

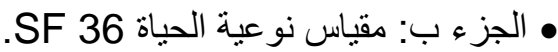

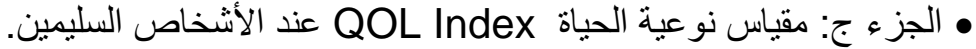

$$
\begin{aligned}
& \text { • الجزء د: وهو عبارة عن رأيك في مقاييس نوعية الحياة. }
\end{aligned}
$$

يتألف الإستبيان من أسئلة متنتوعة. وإذا احتجت الى مساحة إضافية للإجابة الرجاء الكتابـة على خلف الصناء الصفحة.

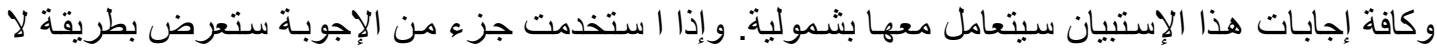

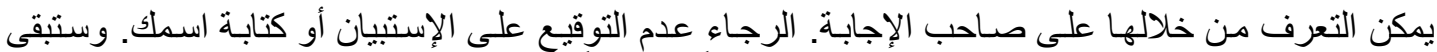

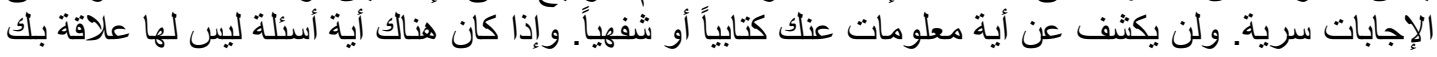

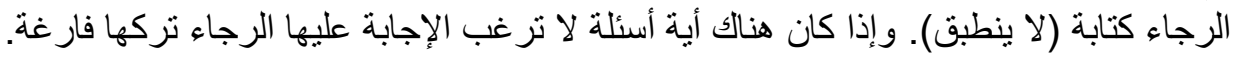

\section{الجزء أ: مطومات أولية}

هذا الجزء من الإسنبيان يطلب منك فيه الإجابة على أسئلة عن معلومات أولية عن نوعية حياتك وصحتك.

$$
\text { 1. ما هي أثمن ثلاثة أثنياء في الحياة يعتبر ها سكان دولة الإمار ات العربية المتحدة ؟ }
$$

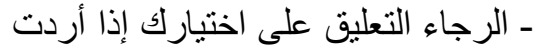


ـ ـ الرجاء التعليق على اختيارك إذا أردت

3. أذكر ثلاثة أثشياء تعتبر ها مهمة في تحسين نوعية حياتك؟ - أ

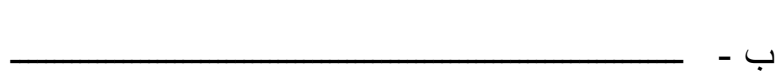

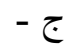
ـ الرجاء التعليق على اختبارك إذا أردت - م

4.

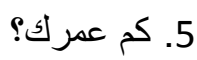

6. هل تعاني من أية أمراض صحية مزمنة غير الفشل الكلوي مثل السكري، أمراض القلب، الربو ، السرطان، التهاب

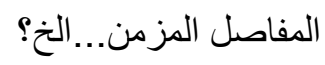
- إذا كانت لإجابة نعم، الرجاء ذكر ها سنة 7. ما هي عدد السنوات التي عشتها في الإمارات؟ شهر

8. حسب علمك ما هو سبب الفنشل الكلوي عندك؟

9. كيف تصف نفسك؟

$$
\begin{aligned}
& \text { ( ) ( ) (إماراتي } \\
& \text { ( ) عربي (غبر إماراتي) }
\end{aligned}
$$

( ) جنوب شرق آسيا (هندي ، باكستاني ، بنغلاديش....الخ)

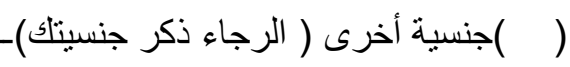

10. ما هي الدرجة العلمية الني أكملتها؟ 


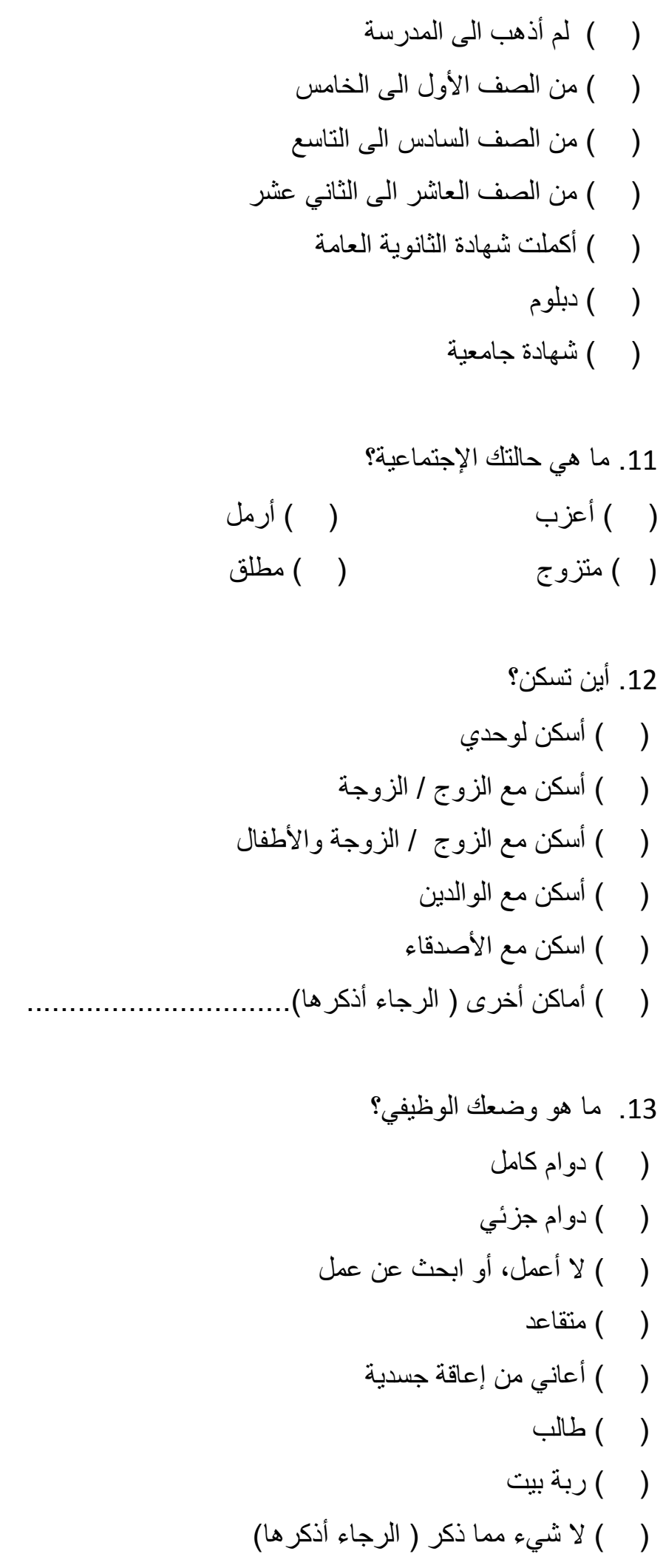




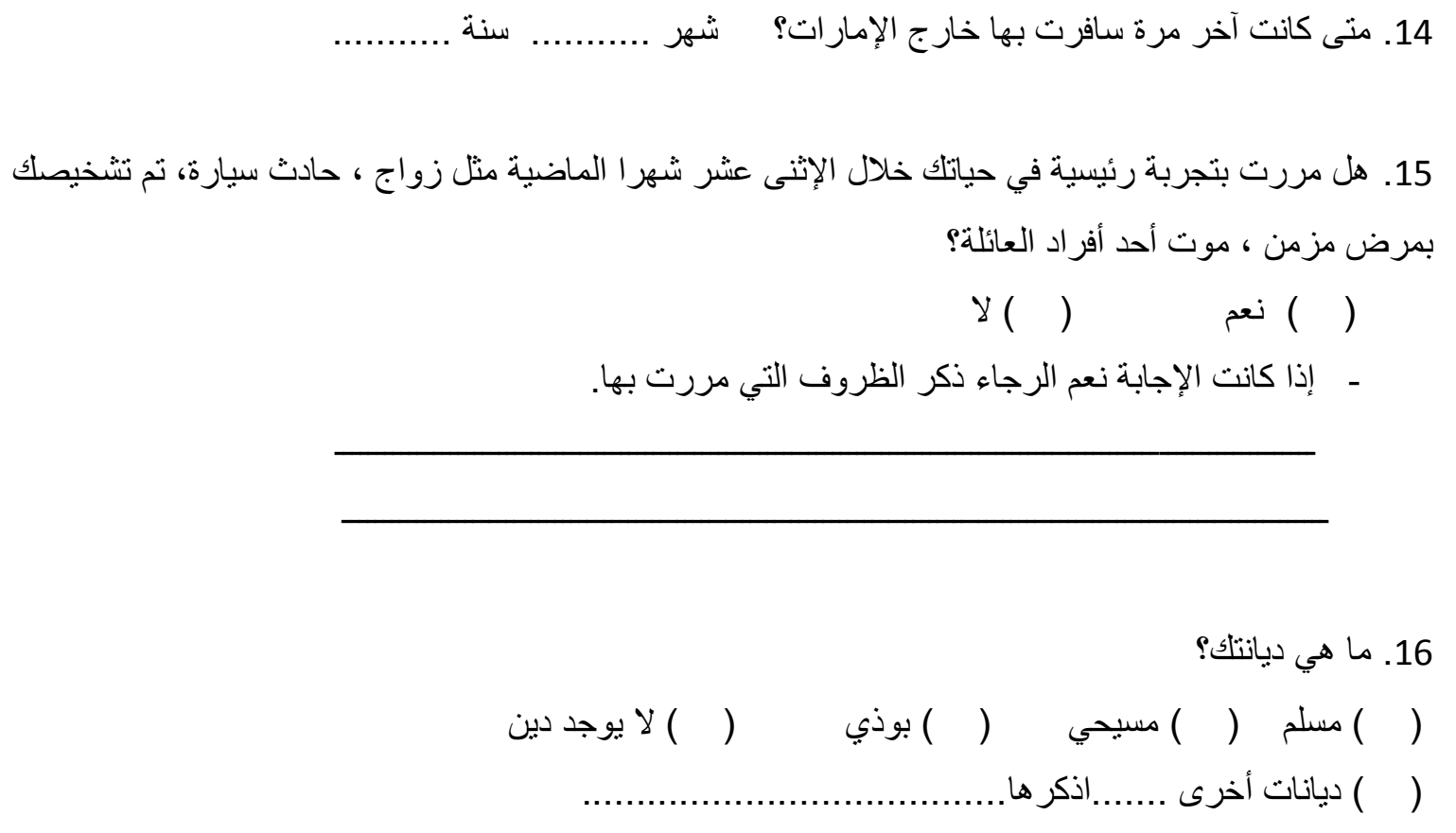

نثكركم لإكمال هذا الجزء والرجاء الإنتقال الى الجزء التالي. 


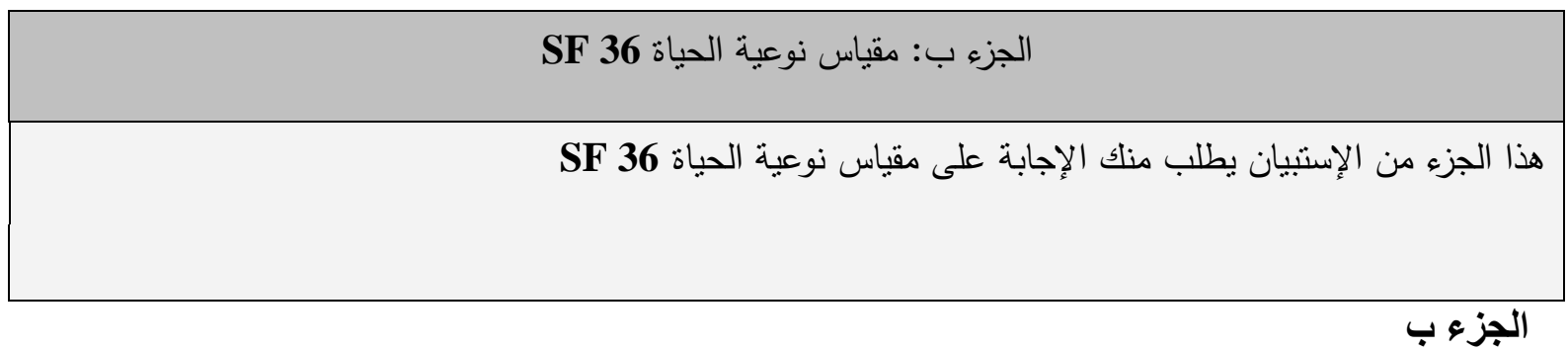

يستفسر هذا الاستبيان عن وجهة نظرك في صحتلك، هذه المعلومات سوف تساعد على تتبع ما تشعر به ومدى قدرتك على أداء نشاطاتك المعتادة. نشكرك على الإجابة عن هذه الأسئلة!

لكل سؤال من الأسئلة التالية يرجى وضع علامة ه في المربع الخاص بالإجابة التي تصف بشكل أفضل ما تشعرون

1.

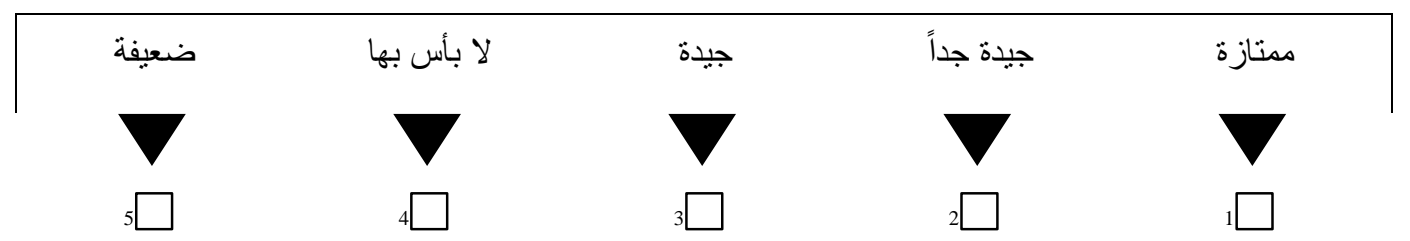

2. مقارنة مع العام الماضي، كيف تقيّم صحتّك الآن بثكل عام؟

\begin{tabular}{|c|c|c|c|c|}
\hline أسو أ بكثير الآن من & أسو أ إلى حد ما & تقريباً مثل العام & أفضل إلى حد ما & أفضل بكثير الآن \\
\hline العام الماضي & الآن من العام & الماضي؟ & الآن من العام & من العام الماضي \\
\hline & الماضي & & الماضي؟ & \\
\hline
\end{tabular}

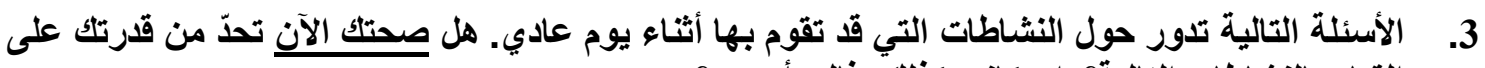

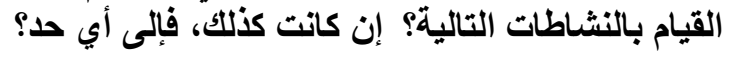




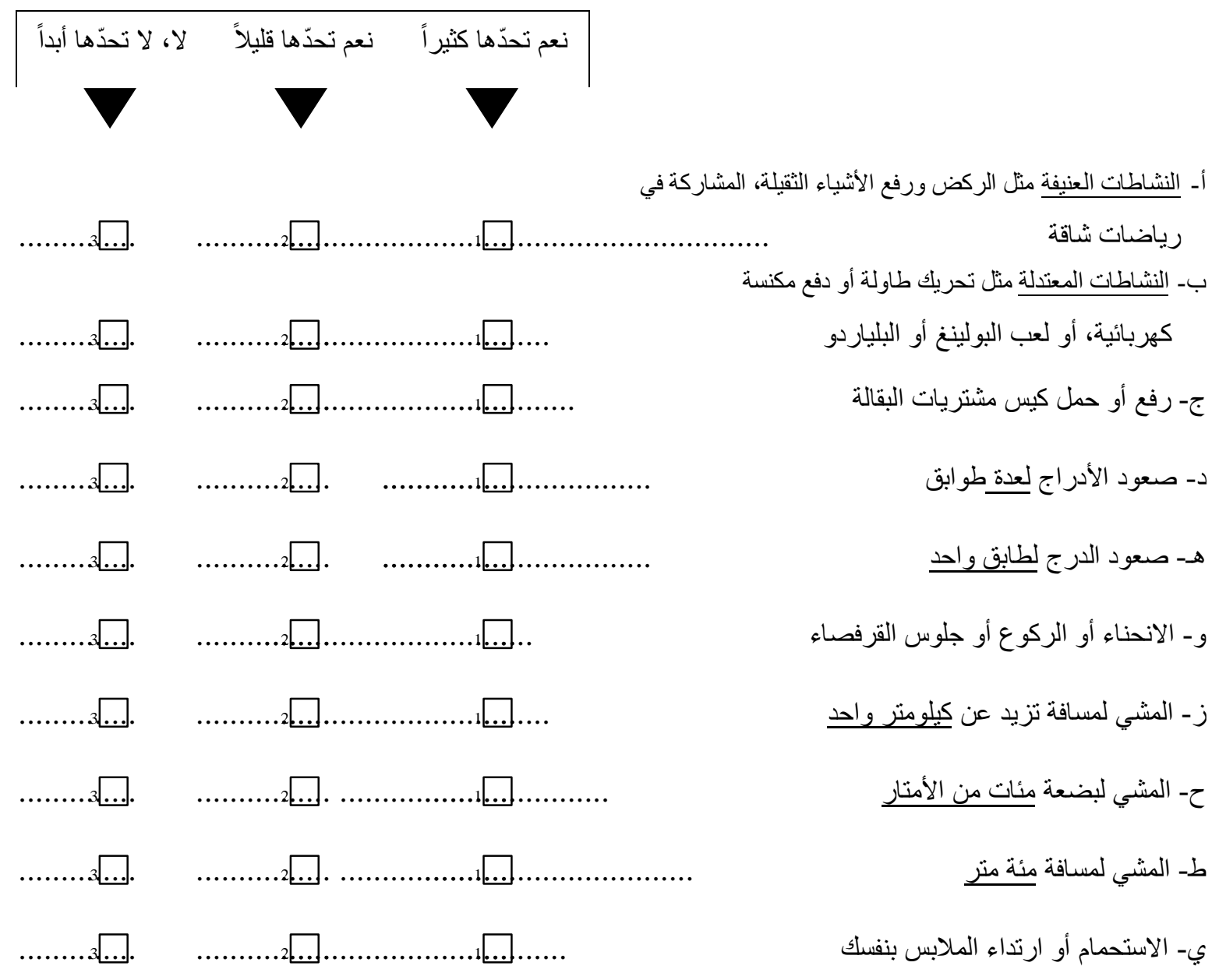


4. خلال الأسابيع الأربعة الماضية، هل حصلت معك أي من المشاكل التالية خلال تأدية عملك أو نشاطاتلك

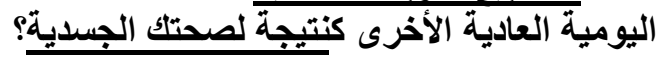

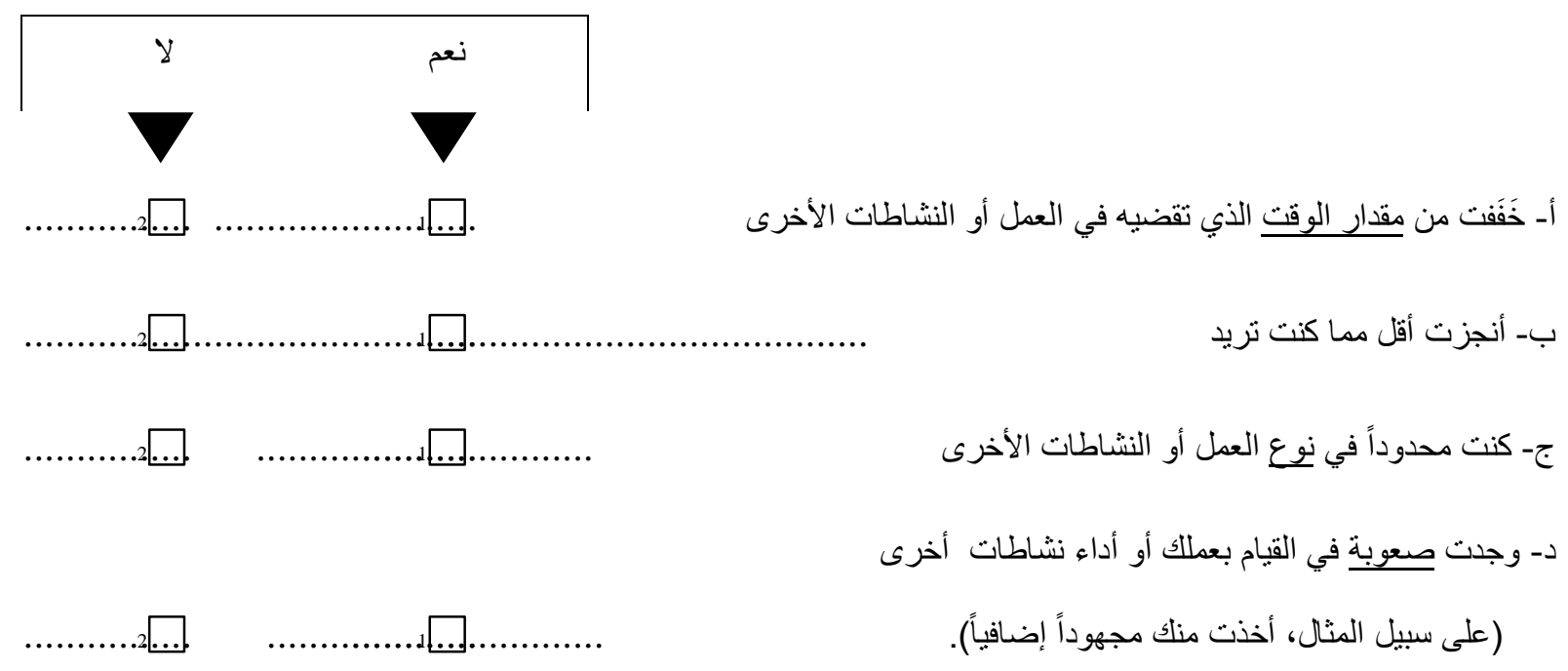

5. خلال الأسابيع الأربعة الماضية، هل حصلت معك أي من المشاكل التالية خلال تأدية عملك أو نشاطاتكا

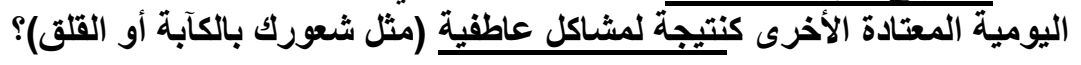

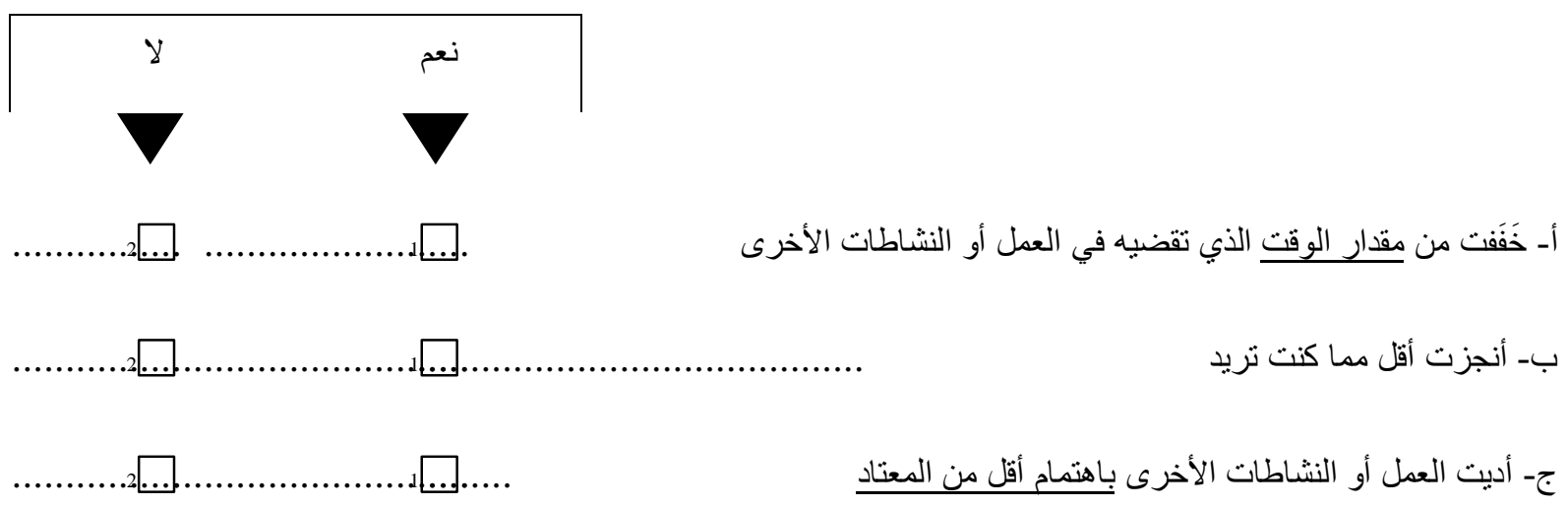




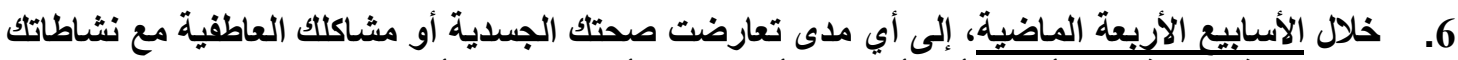

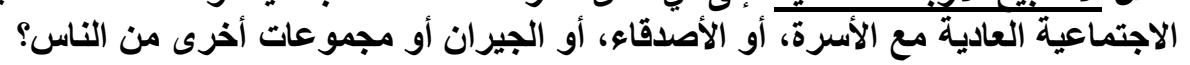

\begin{tabular}{|c|c|c|c|c|}
\hline تعارضت بشكل & تعارضت بشكل & تعارضت بشكل & تعارضت بشكل & لم تتعار ض أبداً \\
\hline كبير جداً & كبير & متوسط & قليل & \\
\hline
\end{tabular}

7. ما مدى شدة الأوجاع الجسدية التي شعرت بها خلال الأسابيع الأربعة الماضيةّ؟

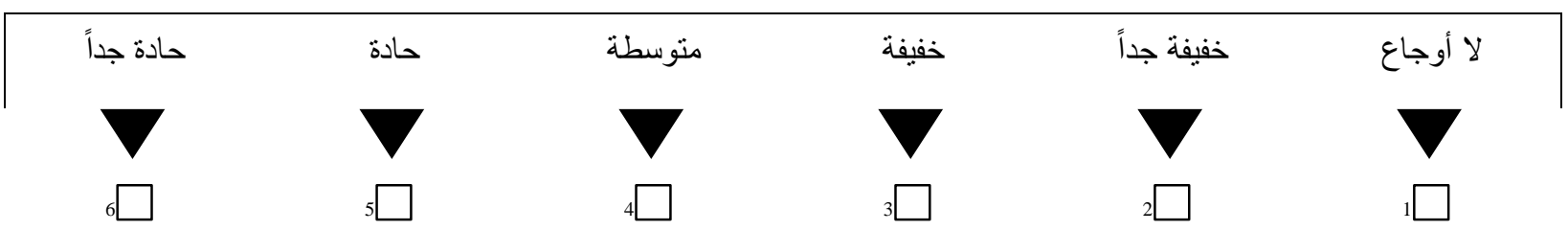

8. خلال الأسبابيع الأربعة الماضية، إلى أي مدى تعارض الألد مع عملك العادي (بما في ذلك عملك خارج

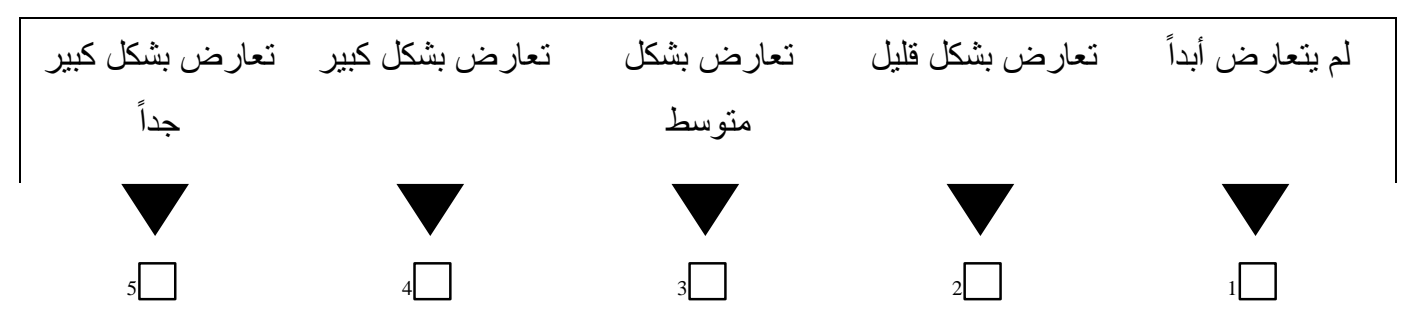


9. هذه الأسئلة تدور حول ما تثنعر به وكيف سارت الأمور معك خلال الأسابيع الأربعة الماضية. الرجاء

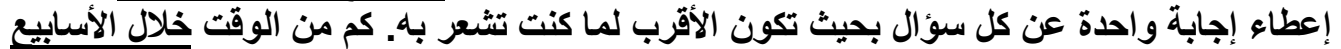

الأربعة الماضية....

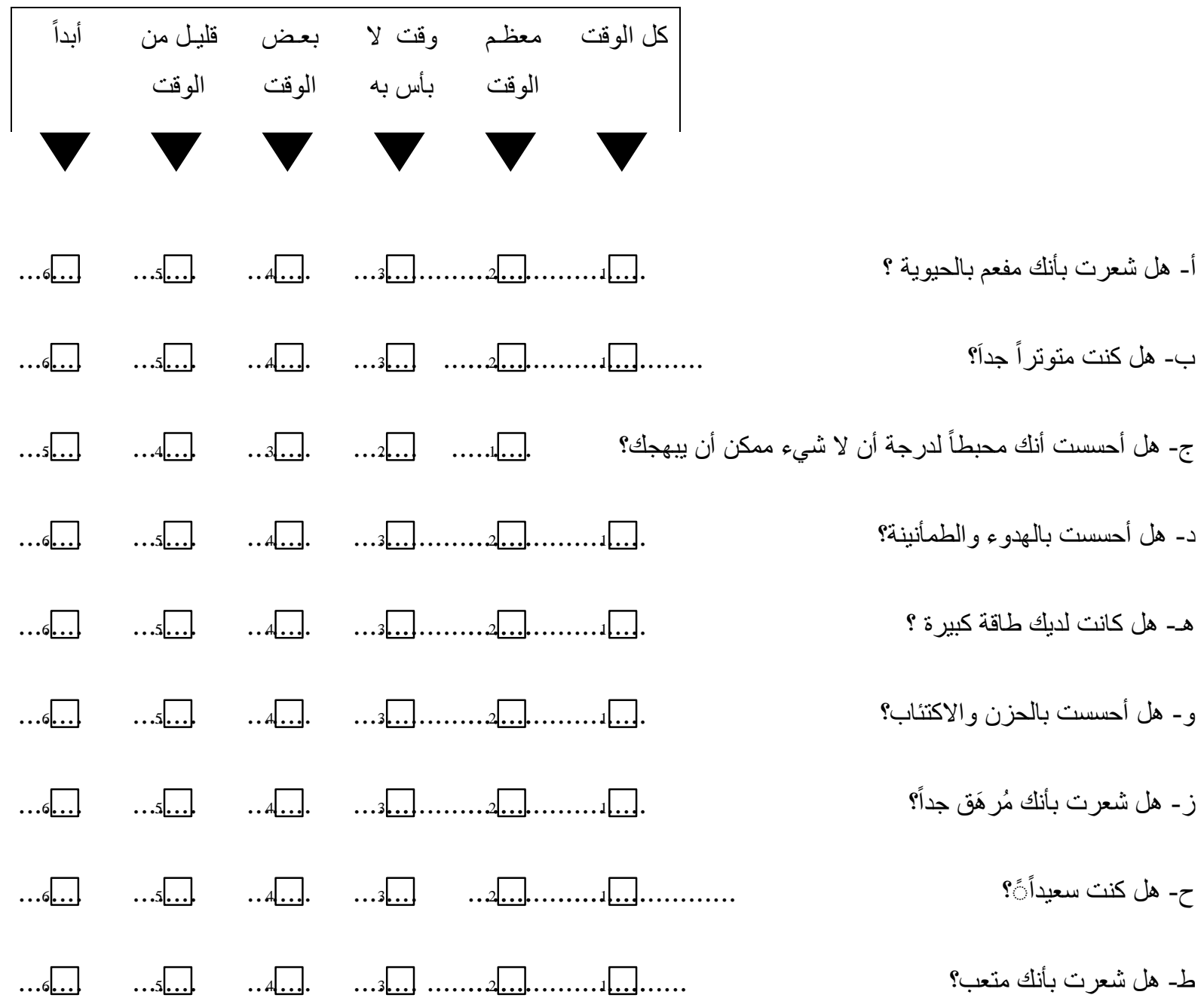


10. خلال الأسابيع الأربعة الماضية، كم من الوقت تعارضت صحتك الجسدية أو مشاكلك العاطفية مع نشاطاتك

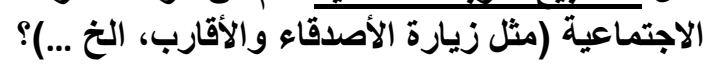

\begin{tabular}{|c|c|c|c|c|}
\hline أبداً & قليل من الوقت & بعض الوقت & معظم الوقت & كل الوقت \\
\hline
\end{tabular}

11. ما مدى صحة أو خطأ كل عبارة من العبارات التالية بالنسبة للك؟

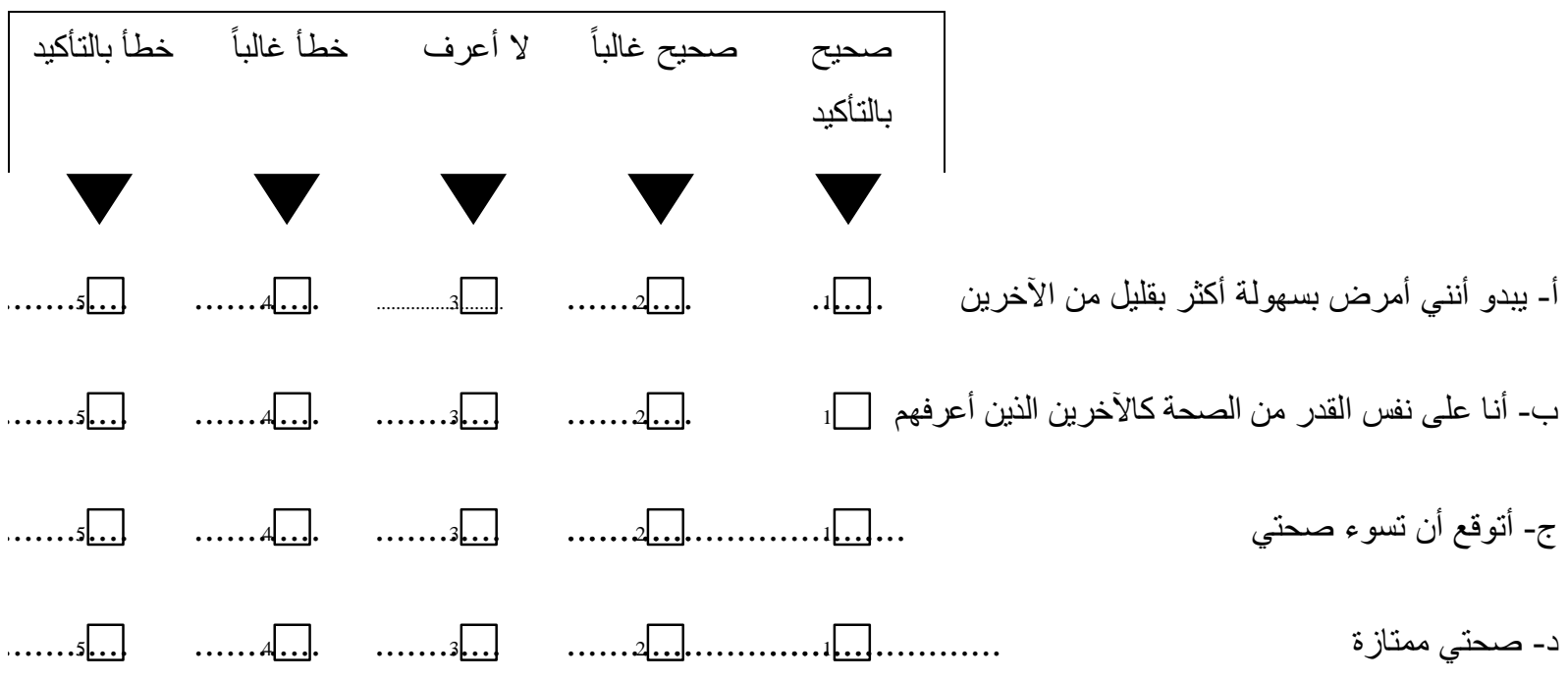

شكراً على الإجابة عن هذه الأسئلة ! 


\section{SF 36 أسئلة تخص رأيك في إستبيان}

1. هل تعتقد بأن هذا الإستييان يتمانشى مع معتقداتك وحضارتك؟؟

$$
\text { [ ] [ ] [ ] [ ] }
$$

2. ما السؤ ال/ الأسئلة التي يجب إضافتها الى هذا الإستبيان لجعله يتمانشى مع معتقدات وحضارة الأشخاص الذين يعيشون في دولة الإمارات العربية المتحدة.

3. ما السؤ ال/ الأسئلة التي يجب حذفها الى هذا الإستبيان لجعله يتمانثى مع معتقدات وحضارة الأشخاص الذين يعيشون في دولة الإمارات العربية المتحدة. 


\section{Quality of Life Index مفَس نويعة الحباة}

(Generic Version- III المهودج (عام)

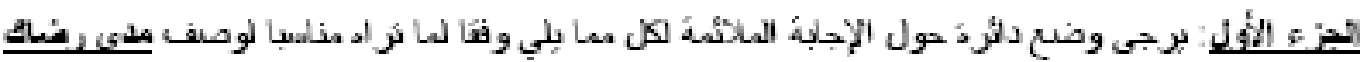

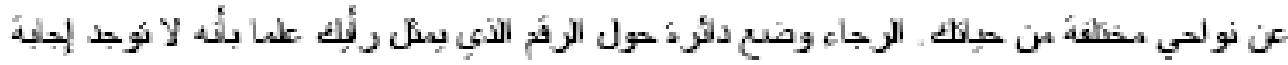
صحبحة أُ خاطئة

\begin{tabular}{|c|c|c|c|c|c|c|}
\hline رلا رل & 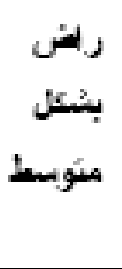 & رلبلِ & فرلير & 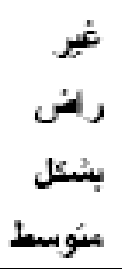 & خبر & كر أثت راضض عن \\
\hline y & $\circ$ & t & $r$ & $r$ & 1 & I, صحتك؟? \\
\hline$y$ & o & i & $r$ & r & 1 & 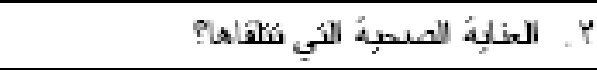 \\
\hline$y$ & o & i & $r$ & r & 1 & 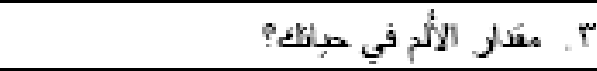 \\
\hline 1 & 。 & i & $r$ & r & 1 & 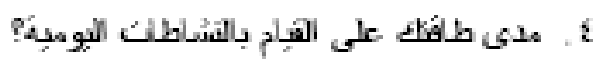 \\
\hline$y$ & 0 & i & $r$ & r & 1 & 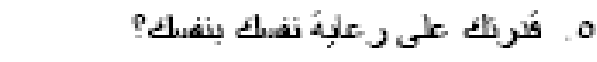 \\
\hline$y$ & o & i & $r$ & r & 1 & 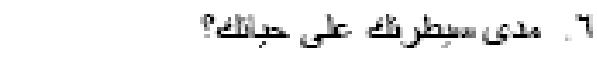 \\
\hline$y$ & 。 & i & $r$ & r & 1 & 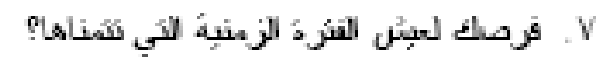 \\
\hline$y$ & $\circ$ & i & $r$ & r & 1 & م. صحة عالتلك؟ \\
\hline$y$ & $\circ$ & i & $r$ & r & 1 & 9 أطفللك? \\
\hline y & 。 & i & $r$ & r & 1 & 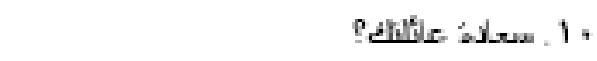 \\
\hline$y$ & 0 & t & $r$ & r & 1 & 11 مبانك الجنسبة \\
\hline$y$ & 0 & t & $r$ & r & 1 & 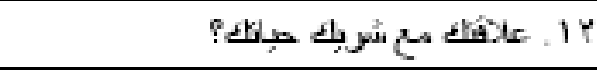 \\
\hline$y$ & 。 & i & $r$ & r & 1 & 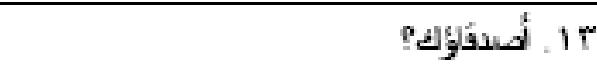 \\
\hline$y$ & 0 & $i$ & $r$ & $r$ & 1 & 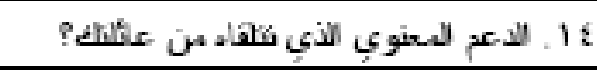 \\
\hline$y$ & 0 & i & $r$ & r & 1 & 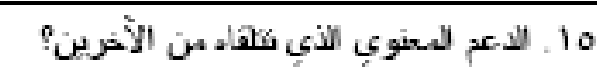 \\
\hline
\end{tabular}

"Quality of Life Indes, Carol Estwing Ferrans \& Marjorie J. Powers (1984 \& 1998) Copyright Arabic translation: Jehnd O. Halabi, University of Jordan, Amman 11942, Jordan, email in iehachalabsayahoo.com 


\begin{tabular}{|c|c|c|c|c|c|c|}
\hline ناندا & مبرَّل مبرل & فَاليلان & 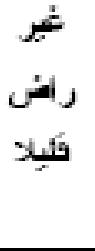 & مُشِّر & رنغ & كر أتك ر اضض عن: \\
\hline$y$ & 0 & t & $r$ & $r$ & 1 & 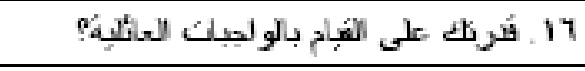 \\
\hline$y$ & $\circ$ & t & $r$ & r & 1 & 1V ف فئسك لاخخرين? \\
\hline$y$ & 0 & t & $r$ & r & 1 & 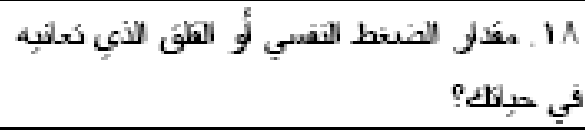 \\
\hline$y$ & 0 & i & r & r & 1 & 19 ه جلير اتلك؟ \\
\hline$y$ & 0 & t & $r$ & r & 1 & 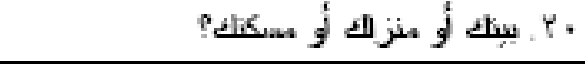 \\
\hline$y$ & 0 & i & $r$ & r & 1 & 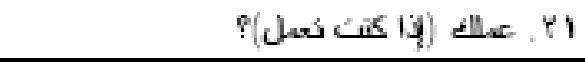 \\
\hline$y$ & 0 & t & $r$ & r & 1 & 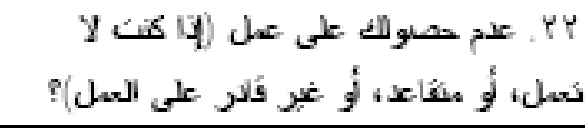 \\
\hline$y$ & 0 & t & $r$ & r & 1 & بَץ. نحصبلٍ العلمي؟ \\
\hline$y$ & 0 & t & $r$ & r & 1 & 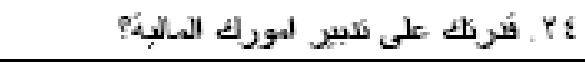 \\
\hline y & $\circ$ & $\mathrm{t}$ & $r$ & r & 1 & 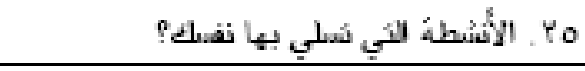 \\
\hline$y$ & 0 & t & $r$ & r & 1 & 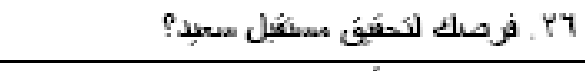 \\
\hline$y$ & $\circ$ & $\mathrm{t}$ & $r$ & r & 1 & 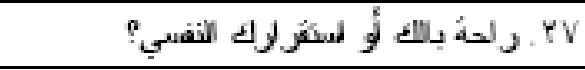 \\
\hline$y$ & 0 & $t$ & $r$ & r & 1 & ^ץ ، إيدانك بالة عز وجل؟ \\
\hline$y$ & 0 & $t$ & $r$ & $r$ & 1 & 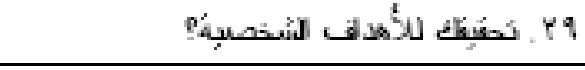 \\
\hline$y$ & 0 & $t$ & $r$ & $r$ & 1 & 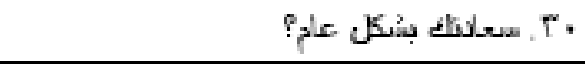 \\
\hline$y$ & 0 & $t$ & $r$ & $r$ & 1 & 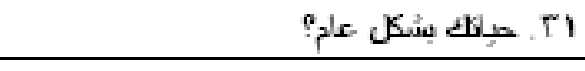 \\
\hline$y$ & 0 & $t$ & $r$ & $r$ & 1 & 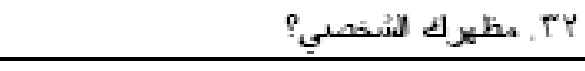 \\
\hline$y$ & 0 & $t$ & $r$ & $r$ & 1 & 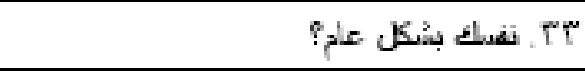 \\
\hline
\end{tabular}

Copyright Arabic translation: Jehad O. Halabi, University of Jordan, Amman, emsil: dr jehndhalabi avahoo.com 


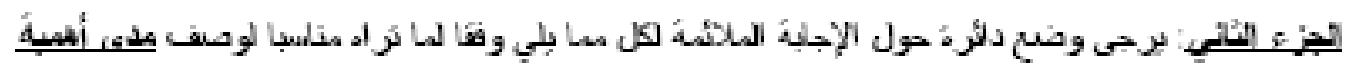

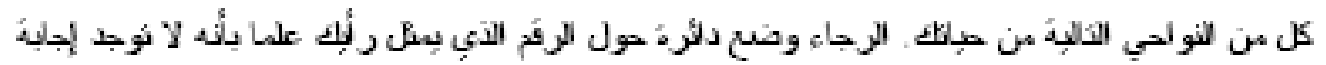

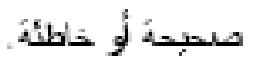

\begin{tabular}{|c|c|c|c|c|c|c|}
\hline مه جثأ & منسكل & فيلע & ثَبر & 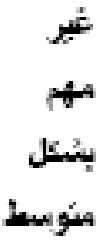 & شهر & 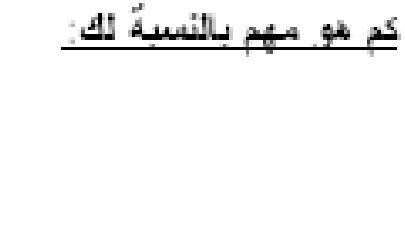 \\
\hline ч & 0 & $\varepsilon$ & $r$ & $r$ & 1 & 1. صحتك؟ \\
\hline 4 & 0 & $\mathfrak{\varepsilon}$ & $r$ & $\varphi$ & 1 & 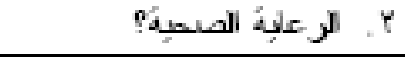 \\
\hline 4 & 0 & $\varepsilon$ & $r$ & $\varphi$ & 1 & 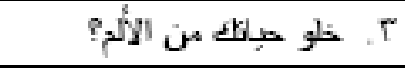 \\
\hline 4 & 0 & $\varepsilon$ & $r$ & $r$ & 1 & 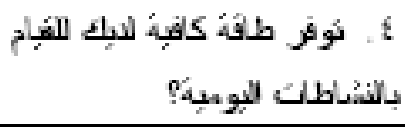 \\
\hline
\end{tabular}

\begin{tabular}{|c|c|c|c|c|c|c|}
\hline ч & $\circ$ & $\varepsilon$ & $r$ & $r$ & 1 & 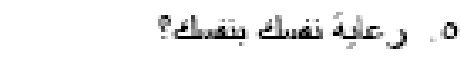 \\
\hline y & $\circ$ & $\varepsilon$ & $r$ & $\varphi$ & 1 & 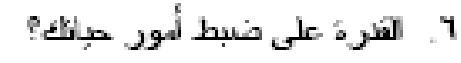 \\
\hline$y$ & o & $\varepsilon$ & $r$ & $\varphi$ & 1 & 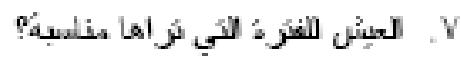 \\
\hline y & $\circ$ & $\varepsilon$ & $r$ & $r$ & 1 & 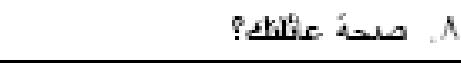 \\
\hline$y$ & 0 & $\varepsilon$ & $r$ & $r$ & 1 & 9 \\
\hline y & $\circ$ & $\varepsilon$ & $r$ & $\varphi$ & 1 & 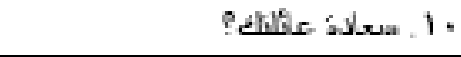 \\
\hline 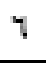 & $\circ$ & $\varepsilon$ & $r$ & $r$ & 1 & 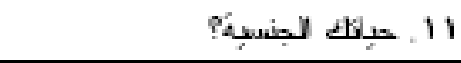 \\
\hline 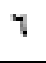 & $\circ$ & $\varepsilon$ & $r$ & $\varphi$ & 1 & 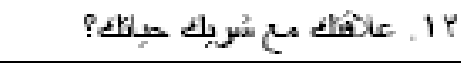 \\
\hline$y$ & $\circ$ & $\varepsilon$ & $r$ & $\varphi$ & 1 & 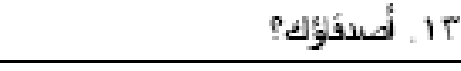 \\
\hline$y$ & $\circ$ & $\varepsilon$ & $r$ & $\varphi$ & 1 & 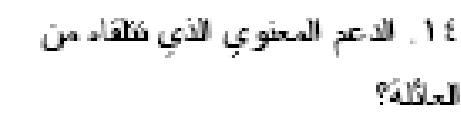 \\
\hline$y$ & 0 & $\varepsilon$ & $r$ & $r$ & 1 & الأخرين? الدم الدعنوي للاذي شلقاد من \\
\hline
\end{tabular}

Quality of Life Index, Carol Estwing Ferrans \& Marjorie J. Powers (1984 \& 1998)

Copyright Arabic translation: Jehad O. Halabi, University of Jordan, Anman, email: dr jehadhalabiavahoecom 


\begin{tabular}{|c|c|c|c|c|c|c|}
\hline مهم جلا & منَسكل & كهيل & غَّنر & 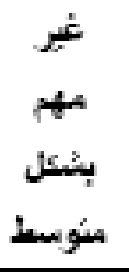 & تشر & 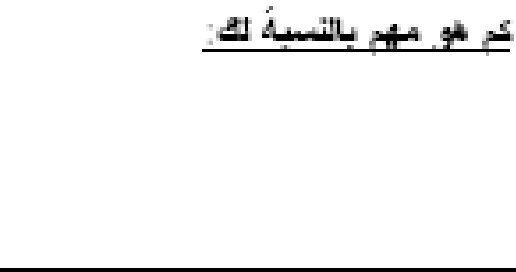 \\
\hline 4 & $\circ$ & $\varepsilon$ & $r$ & $\varphi$ & 1 & 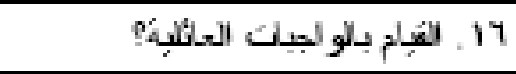 \\
\hline$y$ & 0 & 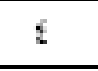 & $r$ & $r$ & 1 & 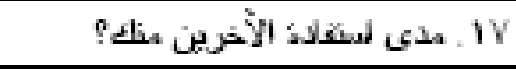 \\
\hline 4 & o & $\varepsilon$ & $r$ & $\varphi$ & 1 & 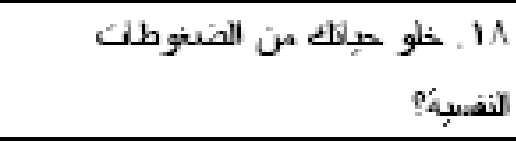 \\
\hline 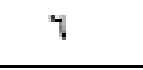 & $\circ$ & $\varepsilon$ & $r$ & $\varphi$ & 1 & 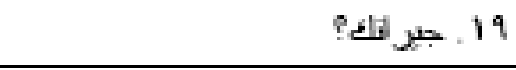 \\
\hline$y$ & 0 & $\varepsilon$ & $r$ & $\varphi$ & 1 & 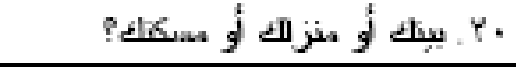 \\
\hline 4 & $\circ$ & $\varepsilon$ & $r$ & $\varphi$ & 1 & 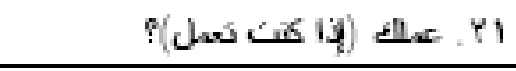 \\
\hline$y$ & 0 & $\varepsilon$ & $r$ & $\varphi$ & 1 & 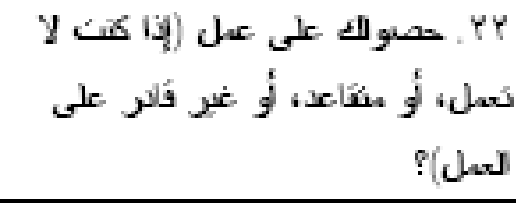 \\
\hline 4 & $\circ$ & $\varepsilon$ & $r$ & $r$ & 1 & r ب . كمصبلٍ العلدي؟ \\
\hline 4 & $\circ$ & $\varepsilon$ & $r$ & $\varphi$ & 1 & 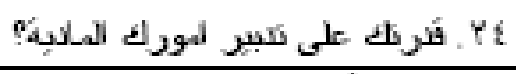 \\
\hline 7 & $\circ$ & $\varepsilon$ & $r$ & $r$ & 1 & 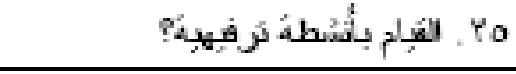 \\
\hline$y$ & o & $\varepsilon$ & $r$ & $\varphi$ & 1 & 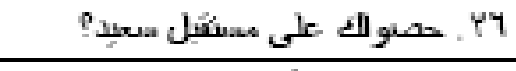 \\
\hline$y$ & o & $\varepsilon$ & $r$ & $\varphi$ & 1 & 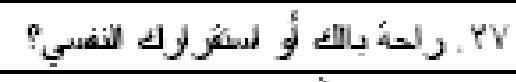 \\
\hline 4 & $\circ$ & $\mathfrak{\varepsilon}$ & $r$ & $r$ & 1 & مب إيدانك بالة عز وجل؟ \\
\hline 4 & o & $\varepsilon$ & $r$ & $\varphi$ & 1 & 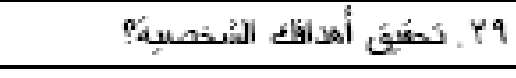 \\
\hline 4 & o & $\varepsilon$ & $r$ & $r$ & 1 & . r. r. r. \\
\hline 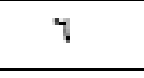 & o & $\varepsilon$ & $r$ & $\varphi$ & 1 & 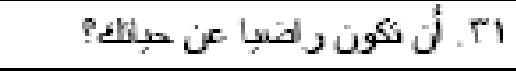 \\
\hline$y$ & o & $\varepsilon$ & $r$ & $\varphi$ & 1 & 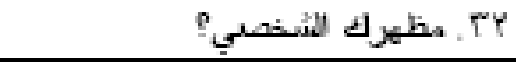 \\
\hline 4 & o & $\varepsilon$ & $r$ & $\varphi$ & 1 & 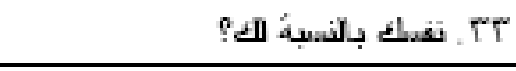 \\
\hline
\end{tabular}

Copyright Arabic translation: Jehad O. Halabi, University of Jordan, Ammon, email: de jehadhalabia yahoecom 


\title{
أسئلة تخص رأيك في إستبيان مقياس نوعية الحياة
}

\author{
1. هل تعتقد بأن هذا الإستبيان يتمانشى مع معتقداتك وحضارتك؟؟ \\ ن ن \\ 2. ما السؤ ال/ الأسئلة التي يجب إضافتها الى هذا الإستبيان لجعله يتمانشى مع معتقدات وحضارة الأشخاص \\ الذين يعيشون في دولة الإمارات العربية المتحدة. \\ 3. ما السؤ ال/ الأسئلة التي يجب حذفها الى هذا الإستبيان لجعله يتمانشى مع معتقدات وحضارة الأشخاص \\ الذين يعيشون في دولة الإمارات العربية المتحدة.
}




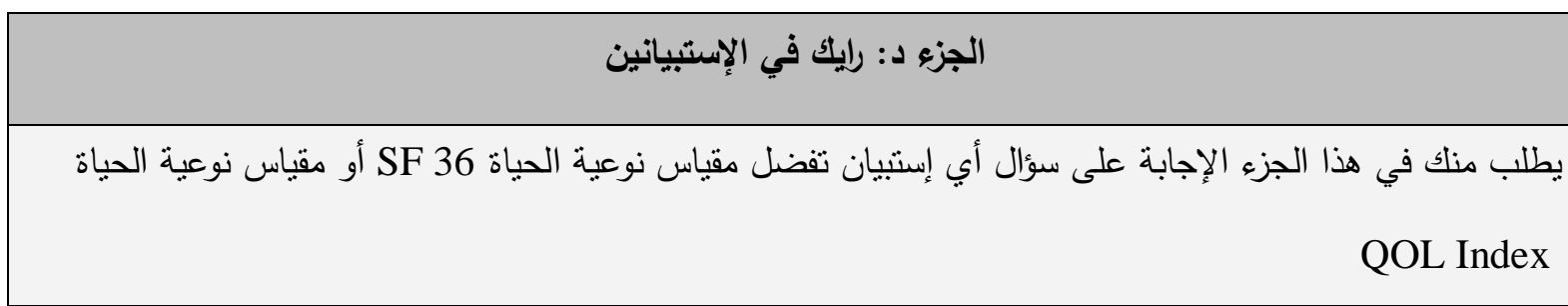

$$
\begin{aligned}
& \text { مقارنة بين الإستبياتين } \\
& \text { أي من الإستبيانين تعتبره ينماثشى مع معتقداتك وحضارتك؟ بلكئ }
\end{aligned}
$$

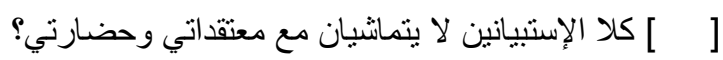

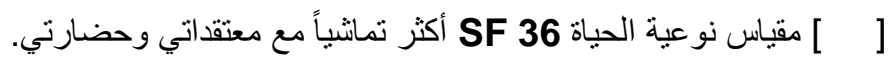

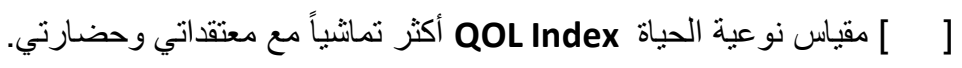

$$
\begin{aligned}
& \text { ] [ ] [ الإستبيانين يتمانثيان مع معتقداتي وحضارني. }
\end{aligned}
$$

\section{نشكركم لإكمال هذا الإستبيان}

الرجاء وضع الإجابات داخل المغلف المرفق وتسليمه للسيد عبد الباسط أيوب أو إرساله على عنوان البريد التالي: وحدة الكلى، مدينة الثيخ خليفة الطبية، صندوق بريد 51900، أبو ظبي، دولة الإمارات العربية المتحدة. 
Appendix 9 Table A1 missing data for the SF-36

Tables A1. Missing data for SF36 tool in the Community sample

\begin{tabular}{|c|c|c|}
\hline & Question Content & $n(\%)$ \\
\hline Sf3 & $\begin{array}{l}\text { Vigorous activities, such as running, lifting heavy objects, participating in } \\
\text { strenuous sports. }\end{array}$ & $3(1.1)$ \\
\hline Sf4 & $\begin{array}{l}\text { Moderate activities, such as moving a table, pushing a vacuum cleaner, } \\
\text { bowling, or playing golf }\end{array}$ & $1(0.4)$ \\
\hline Sf5 & Lifting or carrying groceries & $1(0.4)$ \\
\hline Sf6 & Climbing several flights of stairs & $1(0.4)$ \\
\hline Sf7 & Climbing one flight of stairs & $2(0.7)$ \\
\hline Sf8 & Bending, kneeling, or stooping & $1(0.4)$ \\
\hline Sf10 & Walking several blocks & $2(0.7)$ \\
\hline Sf11 & Walking one block & $2(0.7)$ \\
\hline Sf12 & Bathing or dressing yourself & $2(0.7)$ \\
\hline Sf15 & Were limited in the kind of work or other activities & $1(0.4)$ \\
\hline Sf16 & $\begin{array}{l}\text { Had difficulty performing the work or other activities (for example, it took } \\
\text { extra effort) }\end{array}$ & $2(0.7)$ \\
\hline Sf20 & $\begin{array}{l}\text { Emotional problems interfered with your normal social activities with } \\
\text { family, friends, neighbours, or samples? }\end{array}$ & $1(0.4)$ \\
\hline Sf21 & How much bodily pain have you had during the past 4 weeks? & $1(0.4)$ \\
\hline Sf22 & $\begin{array}{l}\text { During the past } 4 \text { weeks, how much did pain interfere with your normal } \\
\text { work (including both work outside the home and housework)? }\end{array}$ & $2(0.7)$ \\
\hline Sf23 & Did you feel full of pep? & $2(0.7)$ \\
\hline Sf25 & Have you felt so down in the dumps that nothing could cheer you up? & $1(0.4)$ \\
\hline Sf28 & Have you felt downhearted and blue? & $1(0.4)$ \\
\hline Sf29 & Did you feel worn out? & $1(0.4)$ \\
\hline
\end{tabular}




\begin{tabular}{|l|l|r|}
\hline Sf32 & $\begin{array}{l}\text { During the past 4 weeks, how much of the time has your physical health } \\
\text { or emotional problems interfered with your social activities (like visiting } \\
\text { with friends, relatives, etc.)? }\end{array}$ & $3(1.1)$ \\
\hline Sf33 & I seem to get sick a little easier than other people & $1(0.4)$ \\
\hline Sf34 & I am as healthy as anybody I know & $2(0.7)$ \\
\hline Sf35 & I expect my health to get worse & $1(0.7)$ \\
\hline Sf36 & My health is excellent & $1(0.4)$ \\
\hline
\end{tabular}




\section{References}

Abdussalam, M. (2001). Just for husbands! American Muslim, 2(4), 28-29.

Acaray, A., \& Pinar, R. (2005). Quality of life in Turkish haemodialysis patients. International Urology and Nephrology Journal, 37(3), 595-602.

Addington-Hall, J., \& Kalra, L. (2001). Measuring quality of life: Who should measure quality of life? British Medical Journal, 322 (7299), 1417-1420.

Adib, S.M. (2004). From the biomedical model to the Islamic alternative: A brief overview of medical practices in the contemporary Arab world. Social Science \& Medicine, 58(4), 697-702.

Affleck, G., Tennen, H., Keefe, F.J., Lefebvre, J.C., Kashikar-Zuck, S. \& Wright, K. (1999). Everyday life with osteoarthritis or rheumatoid arthritis: Independent effects of disease and gender on daily pain, mood, and coping. Pain, 83(3), 601-609.

Agrawal, V, Garimella, P.S, Roshan, S.J, \& Ghosh, A.K. (2009). Questionnaire survey of physicians: Design and practical use in nephrology. Indian Journal of Nephrology, 19(2), 41-47.

Ahmed, A.S. (1999). Islam Today - A short introduction to the Muslim world. I.B. Tauris, London.

Aiken, L. R. (1996). Rating scales and checklists: Evaluating behavior, personality, and attitudes. New York: John Wiley \& Sons.

Albrecht, G.L., \& Devlieger, P.J. (1999). The disability paradox: High quality of life against all odds. Social Science and Medicine Journal, 48(8), 977-988.

Al-Ghazzali. (1970). The mysteries of purity. Ashraf Press, Lahore.

Altintepe, L., Levendoglu, F., Okudan, N., Guney, I., Savas Cilli, A., Ugurlu, H., et al. (2006). Physical disability, psychological status, and health-related quality of life in older hemodialysis patients and age-matched controls. Hemodialysis International Journal, 10(3), 260-266.

Andresen, E.M., \& Meyers, A.R. (2000). Health-related quality of life outcomes measures. Archives of Physical Medicine and Rehabilitation, 81(12 Suppl. 2), S30-S45.

Amarantos, E., Martinez, A., \& Dwyer, J. (2001). Nutrition and quality of life in older adults. The Journal of Gerontology, 56(2), 54-64.

Anderson, K.L., \& Burckhardt, C.S. (1999). Conceptualization and measurement of quality of life as an outcome variable for healthcare intervention and research. Journal of Advanced Nursing, 29(2), 298-306. 
Andresen, E.M., \& Meyers, A.R. (2000). Health-related quality of life outcomes measures, Archives of Physical Medicine and Rehabilitation, 81(2), S30-S45.

Anderson, R.T., Aaronson, N.K., Bullinger, M. \& McBee, W.L. (1996). A review of the progress towards developing health-related quality-of-life instruments for international clinical studies and outcomes research. Pharmacoeconomics Journal, 10(4),336-355.

Angel, R. \& Cronfein, W. (1988). The use of subjective information on statistical models. American Sociology Review, 53(3), 464-473.

Angel, R., \& Lumpkin, J. (1992). How accurate are the time use reports? Effect of cognitive enhancement and cultural differences on recall accuracy. Applied Cognitive Psychology, 6 (1), 141-159.

Angel, R., \& Thoits, P. (1987). The impact of culture on the cognitive structure of illness. Journal of Culture Medical Psychiatry, 11(3), 465-469.

ANZDATA Registry Report. (2004). Annual report of Australia New Zealand Dialysis Data Registry Report. Retrieved 7 January 2004 from http://www.anzdata.org.au/anzdata/AnzdataReport/download.htm\#27th\%20Report

Apolone, G., \& Mosconi, P. (1998). The Italian SF-36 Health Survey: Translation, validation and norming. Journal of Clinical Epidemiology, 51(11), 1025-1036.

Asadi-Lari, M., Packham, C., \& Gray, D. (2003). Is quality of life measurement likely to be a proxy for health needs assessment in patients with coronary artery disease? Health and Quality of Life Outcomes, Retrieved $16^{\text {th }}$ October 2010 from http://www.hqlo.com/content/1/1/50

Athar, S. (1995). Health concerns for believers: Contemporary issues. Ethical decisionmaking in patient care: An Islamic perspective. The Library of Islam, Illinois.

Awad, A., Denic, S., \& El-Tajic, H. (2008). Validation of the European Organization for Research and Treatment of Cancer Quality of Life Questionnaires for Arabicspeaking populations. Annals of the New York Academy Sciences, 1138(1), 146-154.

Ayers, K.M., Thomson, W.M., Al-Hassiny, H., Rich, A.M., \& Newton, J.T. (2008). A qualitative investigation of the experiences of immigrant dentists working in New Zealand. New Zealand Dental Journal, 104(3), 97-103.

Bachman, J.G., O'Malley, P.M., \& Johnston, L.D. (1984). Drug use among young adults: The impacts of role status and social environment. Journal of Personality and Social Psychology, 47(3), 629-645.

Bakewell, A., Higgins, R.M., \& Edmunds, M.E. (2002). Quality of life in peritoneal dialysis patients: Decline over time and association with clinical outcomes. Kidney International, 61(1), 239-248. 
Barhem, B., Younies, H., \& Muhamad, R. (2009). Religiosity and work stress coping behavior of Muslim employees. Journal of Education, Business and Society: Contemporary Middle Eastern Issues, 2(2), 123-137.

Barnett, V. (1991). Sample survey: principles and methods. London: Edward Arnold.

Bates MS, Edwards WT, Anderson KO. (1993). Ethnocultural influences on variation in chronic pain perception. Pain, 52(1), 101-112.

Beanlands, H., Horsburgh, M.E., Fox, S., Howe, A., Locking-Cusolito, H., et al. (2005). Caregiving by family and friends of adults receiving dialysis. Nephrology Nursing Journal, 32(6), 621-631.

Beer, J. (1995). Body image of patients with ESRD and following renal transplantation. British Journal of Nursing, 4(10), 591-598.

Belbeisi, A., Zindah, M., Walke, H.T., Jarrar, B., \& Mokdad, AH. (2009). Health related quality of life measures by demographics and common health risks, Jordan 2004. International Journal of Public Health, 54(1), 106-110.

Belasco, A., Barbosa, D., Bettencourt, A.R., Diccini, S., \& Sesso, R. (2006). Quality of life of family caregivers of elderly patients on hemodialysis and peritoneal dialysis. American Journal of Kidney Diseases, 48(6), 955-963.

Bergström, J., \& Lindholm, B. (1998). Malnutrition, cardiac disease and mortality: An integrated point of view - editorial review. American Journal of Kidney Disease, 32(5), 834-841.

Berman, E., Merz, J.F., Rudnick, M., Snyder, R.W., Rogers, K.K., Lee, J., et al. (2004). Religiosity in a hemodialysis population and its relationship to satisfaction with medical care, satisfaction with life, and adherence. American Journal of Kidney Diseases, 44(3), 488-497.

Bernardes, S.F., Keogh, E., \& Lima, M.L. (2008). Bridging the gap between pain and gender research: A selective literature review. European Journal of Pain, 12(4), 427440.

Berthoux, F., Gellert, R., Jones, E., Mendel, S., Valderrabano, F., Briggs, D., et al. (1998). Epidemiology and demography of treated end-stage renal failure in the elderly. From the European Renal Association (ERA-EDTA) Registry. Nephrology Dialysis Transplantation Journal, 13(7), 65-68.

Betts, D. ,\& Crotty, G. (1998). Response to illness and compliance of long-term haemodialysis patients. American Nephrology Nurses Association Journal, 15(2), 96100.

Bishop, M., Chapin, M.H. \& Miller, S. (2008). Quality of life assessment in the measurement of rehabilitation outcome. Journal of Rehabilitation, 74(2), 45-55. 
Bishop, M., \& Alien, C.A. (2003). Epilepsy's impact on quality of life: A qualitative analysis. Epilepsy \& Behavior, 4(3), 226-233.

Bohlke, M., Nunes, D.L., Marini, S.S., Kitamura, C., Andrade, M., \& Von-Gysel, M.P. (2008). Predictors of quality of life among patients on dialysis in southern Brazil. Sao Paulo Medical Journal, 126 (5), 252-256.

Bowling, A., Bond, M., Jenkinson, C., \& Lamping, D.L. (1999). Short Form 36 (SF-36) Health survey questionnaire: Which normative data should be used? Comparisons between the norms provided by the omnibus survey in Britain, the health survey for England and the Oxford healthy life survey. Journal of Public Health Medicine, 21(3), 255-270.

Bowling, A. (1995). What things are important in people's lives? A survey of the public's judgment to inform scales of health related to quality of life. Social Science \& Medicine, 41(10), 1447-1462.

Bradley, C. (2001). Importance of differentiating health status from quality of life, Lancet, 357(9249), 7-8.

Bradley, C., Todd, C., Gorton, T., Symonds, E., Martin, A., \& Plowright, R. (1999). The development of an individualized questionnaire measure of perceived impact of diabetes on quality of life: The ADDQOL. Quality of Life Research, 8(1-2), 79-91.

Braveman, P. (2006). Health disparities and health equity: Concepts and measurement. Annual Review of Public Health, 27(1), 167-194.

Brazier, J.E., \& Roberts, J. (2004). Estimating a preference-based index from the SF-12. Medical Care, 42(9), 851-859.

Brazier, J.E., Tsuchiya, A., Roberts, J., \& Busschbach, J. (2004). A comparison of the EQ5D and the SF-6D across seven patient groups. Health Economics, 13(90), 873-884.

Breiterman-White, R. (2005). Functional ability of patients on dialysis: The critical role of anemia. Nephrology Nursing Journal, 32(1), 79-82.

Breitbart, W., McDonald, M.V., Rosenfeld, B., Passik, S.D., Hewitt, D., Thaler, H. et al. (1996). Pain in ambulatory AIDS patients: Pain characteristics and medical correlates. Pain, 68(2), 315-321.

Buargub, M., Nabulsi, M.F., \& Shafeh, T.A. (2006). Prevalence and pattern of renal osteodystrophy in chronic hemodialysis patients: A cross sectional study of 103 patients. Saudi Journal of Kidney Disease \& Transplantation, 17(3), 401-407.

Burns, N. \& Grove, S. (2003). Understanding nursing research. (3 $3^{\text {rd }}$ ed). Philadelphia, Saunders.

Campos, S.S.J., \& Johnson, T.M. (1990). Cultural considerations. In B. Spilker (Ed.), Quality of life assessment in clinical trials (pp. 163-170). New York: Raven Press. 
Camsari, T., Cavdar, C., Yemez, B., Ozkahya, M., Atabay, G. \& Alkin, T., et al. (1999). Psychosexual function in CAPD and hemodialysis patients. Peritoneal Dialysis International, 19(6), 585-588.

Caiman, K. C. (1984). Quality of life in cancer patients-a hypothesis. Journal of Medical Ethics, 10(3), 124-127.

Casetta, I., Riise, T., Wamme Nortvedt, M., Economou, NT., De Gennaro, R., Fazio, P. et al., (2009). Gender differences in health-related quality of life in multiple sclerosis. Multiple Sclerosis, 15(11), 1339-1346.

CARI. (2003). The Caring for Australians with Renal Impairment (CARI) Guidelines. Retrieved 6 August 2010 from: http://www.kidney.org.au

Carr, AJ., \& Higginson, I.J. (2001). Measuring quality of life: Are quality of life measures patient centred? British Medical Journal, 322(7298), 1357-1360.

Carr, A., Thompson, P., \& Ktrwanf, J. (1996). Quality of life measures. British Journal of Rheumatology, 35(3), 275-281.

Cella, D., (1998). Quality of life. In Holland, J.C. (Ed.), Psychooncology (pp. 1135-1146). Oxford University Press, New York.

Center for Advanced Research on Language Acquisition at University of Minnesota. (2011). Definition of Culture. Retrieved $18^{\text {th }}$ November 2011 from http://www.carla.umn.edu/culture/definitions.html

Cerqueira, J., Moraes, M., \& Glina, S. (2002). Erectile dysfunction: Prevalence and associated variables in patients with chronic renal failure. International Journal of Impotence Research, 14(2), 65-67.

Chertow, G. (1999). Modality-specific nutrition support in ESRD: Weighing the evidence. American Journal of Kidney Disease, 33(1). 193-197.

Cherry, K. (2010). What is Validity? Retrieved on February $26^{\text {th }}, 2010$ from http://psychology.about.com/od/researchmethods/f/validity.htm

Cleary, J. (2005). Quality of life of patients on haemodialysis for end-stage renal disease. Journal of Advanced Nursing, 51(6), 577-586.

Cleary, J., \& Drennan, J. (2005). Quality of life of patients on haemodialysis for end-stage renal disease. Journal of Advanced Nursing, 51(6), 577-586.

Coelho-Marques, F.Z., Wagner, M.B., Poli de Figueiredo, C.E., \& d'Avila, D.O. (2006). Quality of life and sexuality in chronic dialysis female patients. International Journal of Impotence Research, 18(6), 539-543. 
Goh, C.R., Lee, K.S., Tan, T.C., Wang, T.L., Tan, C.H., et al., (1996). Measuring quality of life in different cultures: Translation of the Functional Living Index for Cancer (FLIC) into Chinese and Malay in Singapore. Annals of the Academy of Medicine, Singapore, 25(2), 323-334.

Cook, S. (1995). Psychological and educational support for CAPD patients. British Journal of Nursing, 4(14), 809-810.

Coons, S.J., Rao, S., Keiner, D.L., \& Hays, R.D. (2000). A comparative review of generic quality-of-life instruments. Pharmacoeconomics, 77(1), 13-35.

Covic, A., Seica, A., Gusbeth-Tatomir, P., Gavrilovici, O., \& Goldsmith, D.J. (2004). Illness representations and quality of life scores in haemodialysis patients. Nephrology Dialysis Transplantation Journal, 19(8), 2078-2083.

Creamer, P., Lethbridge-Cejku, M. \& Hochberg, M.C. (1999). Determinants of pain severity in knee osteoarthritis: Effect of demographic and psychosocial variables using 3 pain measures. Journal of Rheumatology, 26(8), 1785-1792.

Cukor, D., Cohen, S., Peterson, R., \& Kimmel, P. (2007). Psychosocial aspects of chronic disease: ESRD as a paradigmatic illness. Journal of the American Society of Nephrology, 18(12), 3042-3055.

Cummins, R.A., McCabe, M., Gullone, E., \& Romeo, Y. (1994). The Comprehensive Quality Of Life Scale (ComQOL): Instrument development and psychometric evaluation on college staff and students. Educational and Psychological Measurement, 54(2), 372-382.

Dao-qi, S. (1990). Features and prospects in the urbanization of rural population in China. Geo Journal, 21(1), 65-69.

Davis, K., Schoenbaum, S., \& Audet, A. (2005). A 2020 vision of patient-centered primary care. Journal of General Internal Medicine, 20(10), 953-957.

DCCT Research Group. (1988). Reliability and validity of a diabetes quality of life measure for the DCCT. Diabetes Care, 11(9), 725-732.

Definition of cultural relevance. (2011). Retrieved 6 August 2010 from: http://www.education.com/definition/cultural-relevance/

Dijkers, M. P. (2005). Quality of life of individuals with spinal cord injury: A review of conceptualization, measurement, and research findings. Journal of Rehabilitation Research and Development, 42(3), 87-102.

Dijkers, M P. (2003). Individualization in quality of life measurement: Instruments and approaches. Archives of Physiology Medical Rehabilitation, 84(4), 3-14. 
Drueke, T.B., \& Eckardt, K.U. (2002). Role of secondary hyperparathyroidism in erythropoietin resistance of chronic renal failure patients. Nephrology Dialysis Transplantation Journal, 17(5), 28-31.

Dunn, S., Lewis, S., Bonner, P., \& Meize-Grochowski, R. (1994). Quality of life for spouses of CAPD patients. American Nephrology Nurses Association Journal, 21(5), 237-246.

Dwyer, J.T., Larive, B., Leung, J., Rocco, M., Burrowes, J.D., \& Chumlea, W.C. (2002). Nutritional status affects quality of life in Hemodialysis (HEMO) Study patients at baseline. Journal of Renal Nutrition, 12(4), 213-223.

Dyson, J., Cobb, M., \& Forman. D. (1997). The meaning of spirituality: A literature review. Journal of Advanced Nursing, 26(6), 1183-1188.

Eady, R. (2008). Survival is not enough: Reflections of a long-term renal patient. Journal of Nephrology, 21(13), S3-S6.

Edlund, M. \& Tancredi, L. (1985). Quality of life: An ideological critique, Perspectives in Biology and Medicine, 28(4), 591-607.

Edwards, P. Roberts, I., Clarke, M., DiGuiseppi, C., Pratap, S., Wentz, R. et al., (2002). Increasing response rates to postal questionnaires: Systematic review. British Medical Journal, 324(7347), 1183-1192.

Edwards, R.R., Doleys, D.M., Fillingim, R.B. \& Lowery, D. (2001). Ethnic differences in pain tolerance: clinical implications in a chronic pain population. Psychosomatic Medicine, 63(2), 316-323.

Eknoyan, G., Beck, G., Cheung, A. \& Daugirdas, J. (2002). Effect of dialysis dose and membrane flux in maintenance hemodialysis. The New England Journal of Medicine, 347(25), 2010-2019.

Elal, G. \& Krespi, M. (1999). Life events, social support and depression in hemodialysis patients, Journal of Community and Applied Social Psychology, 9(5), 23-33.

El-Haddad, M. (2006). Nursing in the United Arab Emirates: A historical background. International Nursing Review, 53(4), 284-289.

El Rhazi, K., Nejjari, C., Serhier, Z., Tachfouti, N., Berraho, M., Zakaria, Y., et al., (2009). Cross-cultural adaptation difficulties in health quality of life scales for developing countries: Example of St-George respiratory questionnaire validation in Morocco. Review Epidemiology Sante Publique Journal, 57(3), 179-189.

Encyclopædia Britannica (2006). History of United Arab Emirates. Retrieved 28 November 2006 from http://www.britannica.com/EBchecked/topic/615441/history-of-UnitedArab-Emirates 
Essink-Bot, M., Stouthard, M. \& Bonsel, G. (1993). Generalizability of valuation on health status collected with the Euro quality of life questionnaire. Journal of Health Economy, 2(2), 237-246.

Fayers, P.M., Hand, D. J., Bjordal, K., \& Groenvold, M. (1997). Causal indicators in quality of life research. Quality of Life Research Journal, 6(5), 393-406.

Fayer, P. \& Sprangers, M. (2002). Understanding self related health. The Lancet, 359(9302), 187-188.

Felce, D. (1997). Defining and applying the concept of quality of life. Journal of Intellectual Disability Research, 41(2), 126-135.

Ferrans, C. (2006). Ferrans and Powers Quality of Life Index. Retrieved $23^{\text {rd }}$ of July 2006 from http://www.uic.edu/orgs/qli/index.htm

Ferrans, C.E. (2000). Quality of life as an outcome of cancer care. In Yarbro, C., Frogge, M. \& Goodman, M., (eds). Cancer nursing: Principles and practice, 5th ed. (pp. 243258). Boston: Jones and Bartlett.

Ferrans, C. (1996). Development of a conceptual model of quality of life. Scholarly Inquiry for Nursing Practice Journal, 10(3), 293-304.

Ferrans C.E., \& Powers M.J. (1993). Quality of life of hemodialysis patients. American Nephrology Nurses Association Journal, 20 (5), 575-581.

Ferrans, C., \& Powers, M. (1992). Psychometric assessment of the Quality of Life Index. Research in Nursing and Health, 15(1), 29-38.

Ferrans, C.E. (1990a). Quality of life: Conceptual issues. Seminars in Oncology Nursing, $6(4), 248-254$.

Ferrans, CE. (1990b). Development of a quality of life index for cancer patients. Oncology Nursing Forum, 17(3), 15-19.

Ferrans, C., \& Powers, M. (1985). Quality of life index: Development and psychometric properties. Advances in Nursing Science, 8(1), 15-24.

Foley, R.N., Parfrey, P.S., \& Sarnak M.J. (1998). Clinical epidemiology of cardiovascular disease in chronic renal disease. American Journal of kidney diseases, 32(3), 112119.

Fox-Rushby, J. \& Parker, M. (1995). Culture and the measurement of health-related quality of life. European Review of Applied Psychology, 45(4), 257-263.

Frequent Hemodialysis Network (FHN) Trial Group, Chertow, G.M., Levin, N.W, Beck, G.J., Depner, T.A. et al., (2010). Incenter hemodialysis six times per week versus three times per week. New England Journal of Medicine, 9363(24), 2287-2300. 
Frick, E., Borasio, G.D., Zehentner, H., Fischer, N., \& Bumeder, I. (2004). Individual quality of life of patients undergoing autologous peripheral blood stem cell transplantation. Psychooncology, 13(2), 116-124.

Fromm, K., Andrykowski, M.A., \& Hunt, J. (1996). Positive and negative psychosocial sequelae of bone marrow transplantation: Implications for quality of life assessment. Journal of Behavioral Medicine, 19(3), 221-240.

Fukuhara, S., Yamazaki, S., Marumo, F., Akiba, T., Akizawa, T., Fujimi, S., et al. (2006). Health-related quality of life of predialysis patients with chronic renal failure. Clinical Practice Journal, 105(1), 1-8.

Garratt, A.M., \& Ruta, D.A. (1999). The patient generated index. In: Joyce, C.R.B., O'Boyle, C.A., McGee, H. (Eds.), Individual quality of life: Approaches to conceptualisation and assessment (pp. 105-118). Harwood Academic Publishers, Amsterdam.

Garratt, A., Schmidt, L., Mackintosh, A., \& Fitzpatrick, R. (2002). Quality of life measurement: Bibliographic study of patient assessed health outcome measures. British Medical Journal, 15324(7351), 1417-1422.

Gencoz, T., \& Astan, G. (2006). Social support, locus of control, and depressive symptoms in hemodialysis patients. Scandinavian Journal of Psychology, 47(3), 203-208.

Geisinger, K. (1994). Cross-cultural normative assessment: Translation and adaptation issues influencing the normative interpretation of assessment instruments. Psychological Assessment Journal, 6(5), 304-312.

Ghezeljeh, T.N., Momtahen, M., Tessma, M.K., Nikravesh, M.Y., Ekman, I., \& Emami, A. (2010). Gender specific variations in the description, intensity and location of Angina Pectoris: a cross-sectional study. International Journal of Nursing Studies, 47(8), 965-974.

Giacaman, R., Khatib, R., Shabaneh, L., Ramlawi, A., Sabri, B. Sabatinelli et al. (2009). Health status and health services in the occupied Palestinian territory. Lancet, 373(9666), 837-850.

Gill, TM., \& Feinstein, A.R. (1994). A critical appraisal of the quality of quality of-life measurements. Journal of American Medical Association, 272(8), 619-626.

Goh, C.R., Lee, K.S., Tan, T.C., Wang, T.L., Tan, C.H., Wong, J. et al, (1996). Measuring quality of life in different cultures: Translation of the Functional Living Index for Cancer (FLIC) into Chinese and Malay in Singapore. Annals of the Academy of Medicine, 25(3), 323-334.

Gonzalez-Calvo, J., Gonzalez, V. M., \& Lorig, K. (1997). Cultural diversity issues in the development of valid and reliable measures of health status. Arthritis Care and Research Journal, 10(6), 448-456. 
Goodkin, D.A., Bragg-Gresham, J.L., Koenig, K.G., Wolfe, R.A., Akiba, T., Andreucci, V.E., et al. (2003). Association of comorbid conditions and mortality in hemodialysis patients in Europe, Japan, and the United States: The Dialysis Outcomes and Practice Patterns Study (DOPPS). Journal of the American Society of Nephrology, 14(12), 3270-3277.

Goyen, M., \& Debatin, J. (2009). Healthcare costs for new technologies. European Journal of Nuclear Medicine and Molecular Imaging, 36(1), 139-143.

Grapsa, I., \& Oreopoulos, DG. (1996). Practical ethical issues of dialysis in the elderly. Seminar on Nephrology, 16(4), 339-352.

Graham, J., Stoebner-May, D., Ostir, G., Soham, A., Peek, M. et al., (2009). Health related quality of life in older Mexican Americans with diabetes: A cross-sectional study. Health and Quality of Life Outcomes, 5(39), 1-7.

Greene, R. (2005). Using the Ferrans and Powers quality of life Index of dialysis: A comparison of quality of life in older and younger African Americans receiving hemodialysis. Topics in Geriatric Rehabilitation, 21(3), 230-232.

Groessl, E., Ganiats, T., \& Sarkin, A. (2006). Sociodempgraphic differences in quality of life in rheumatoid arthritis, Pharmacoeconomics, 24(2), 109-121.

Guyatt, G.H. (1993). The philosophy of health related quality of life translation. Quality of Life Research Journal, 2(6), 461-465.

Guyatt, G.H., Feeny, D.H. \& Patrick, D.L. (1993). Measuring health-related quality of life. Annals of Internal Medicine, 118(8), 622-629.

Gudex, C. (1995). Health related quality of life in end stage renal failure. Quality of Life Research Journal, 4(2), 359-366.

Gudykunst, W. B., \& Kim, Y.Y. (1992). Communicating with strangers: An approach to intercultural communication. New York: McGraw-Hill.

Gusbeth-Tatomir, P., Boisteanu, D., Seica, A., Buga, C., \& Covic, A. (2007). Sleep disorders: A systematic review of an emerging major clinical issue in renal patients. International Urology and Nephrology, 39(4), 1217-1227.

Guyatt, G.H., Feeny, D.H., \& Patrick, D.L. (1993). Measuring health-related quality of life. Annals of Internal Medicine, 118(8), 622-629.

Haas, B.K. (1999). A multidisciplinary concept analysis of quality of life. Western Journal of Nursing Research, 21(6), 728-742.

Hagren, B., Pettersen, I., Severinsson, E., Lutzen, K., \& Clyne, N. (2001). The haemodialysis machine as a lifeline: Experiences of suffering from end-stage renal disease. Journal of Advanced Nursing, 34(2), 196-202. 
Halabi, J. (2006). Psychometric properties of the Arabic version of Quality of Life Index. Journal of Advanced Nursing, 55(5), 604-610.

Halligan, P. (2006). Caring for patients of Islamic denomination: Critical care nurses' experiences in Saudi Arabia. Journal of Clinical Nursing, 15(12), 1565-1573.

Hamilton, G., \& Locking-Cusolito, H. (2003). Hemodialysis adequacy and quality of life: how do they relate? Canadian Association of Nephrology Nurses and Technologists, 13(4), 24-29.

Harrison, J. (1993). Spirituality and nursing practice. Journal of Clinical Nursing. 2(2), 211 217.

Hemingway, H., Stafford, M., Stansfield, S., Shipley, M., \& Marmot, M. (1997). Is the SF-36 a valid measure of change in population health? Results from the Whitehall II study. British Medical Journal, 315(7118), 1273-1279.

Heidenheim, A.P., Muirhead, N., Moist, L. \& Lindsay, R.M. (2003). Patient quality of life on quotidian hemodialysis. American Journal Kidney Diseases, 42(1), 36-41.

Herdman, M., Fox-Rushby, J., \& Badia, X. (1998). A model of equivalence in the cultural adaptation of HRQoL instruments: The universalist approach. Quality of Life Research Journal, 7(4), 323-335.

Hickey, A., Bury, G., O'Boyle, C., Bradely, F., O'Kelly, F., \& Shannon, W. (1996). A new short form individual quality of life measure (SEIQOL-DW): Application in a cohort of individuals with HIV/AIDS. British Medical Journal, 313(7048), 29-33.

Hicks, L.S., Cleary, P.D., Epstein, A.M., \& Ayanian, J.Z. (2004). Differences in healthrelated quality of life and treatment preferences among black and white patients with end-stage renal disease. Quality of Life Research Journal, 13(6), 1129-1137.

Higginson, I. \& Carr, A. (2001). Measuring quality of life: Using quality of life measures in the clinical settings. British Medical Journal, 3220(7297), 1297-1300.

Holder, B. (1997). Family support and survival among African-American end-stage renal disease patients. Journal of Advanced Renal Replacement Therapy, 1(1), 13-21.

Hopman, W. M., Towheed, T., Anastassiades, T., Tenenhouse, A., Poliquin, S., Berger, C., et al. (2000). Canadian normative data for the SF-36 health survey. Canadian multicentre osteoporosis study research group. Canadian Medical Association Journal, 163(3), 265-271.

Hsieh, R., Huang, S., Chen, S., Lin, W., Wu, C., Chang, C., \& Lee, W. (2010). Changes in physical functional performance and quality of life in hemodialysis patients in Taiwan: A preliminary study. Journal of Nephrology, 23 (01), 41-48. 
Hsieh, H.F. \& Shannon, S.E. (2005). Three approaches to qualitative content analysis. Quality of Life Research Journal, 15(9), 1277-1288.

Huang, C., Wu, A., \& Frangakis, C. (2006). Do the SF-36 and WHOQOL-BREF measure the same constructs? Evidence from the Taiwan population. Quality of Life Research Journal, 15(1), 15-24.

Hudson, S. (1995). Nephrology clinical outcomes part one: Mortality, morbidity, adequacy of treatment, and quality of life. American Nephrology Nurses Association Journal, 22(1), 113-121.

Hunt, S. M. (1994). Cross-cultural comparability of quality of life measures. International Symposium on Quality of Life and Health (pp. 25-27). Blackwell Verlag, Berlin.

Hutchful, C. (1980). Psychosocial stressors impacting on adults on hemodialyis. Nephrology Nurse, 2(5), 31-34.

Hyland, M.E. (1999). A reformulation of quality of life for medical science. In: Joyce, C.R.B., O'Boyle, C.A., McGee, H. (Eds.). Individual quality of life: Approaches to conceptualisation and Assessment. Harwood Academic Publishers, Amsterdam. (pp. 41-49).

Iliescu, E.A., Yeates, K.E., \& Holland, D.C. (2004). Quality of sleep in patients with chronic kidney disease. Nephrology Diaysis Transplanation Journal, 19(1), 95-99.

Iliescu, E.A., Coo, H., McMurray, M.H., Meers, C.L., Quinn, M.M., Singer, M.A., et al., (2003). Quality of sleep and health-related quality of life in haemodialysis patients. Nephrology Diaysis Transplanation Journal, 18(1), 126-132.

Ingersoll, G.M., \& Marrero, D.G. (1991). A modified quality of life measure for youths: Psychometric properties. Diabetes Education, 17(2), 114-118.

International Religious Freedom Report. (2005). 2005 Report on International Religious Freedom. Retrieved 28 November 2006 from: http://www.state.gov/g/drl/rls/irf/2005/

Jaber, B.L, \& Madias, N.E. (2005). Progression of chronic kidney disease: Can it be prevented or arrested? American Journal of Medicine, 118(12), 1323-1330.

Jager, KJ., van Dijk, P.C., Dekker, F.W. Stengel, B., Simpson, K., Briggs, J.D., \& ERAEDTA Registry Committee. (2003). The epidemic of aging in renal replacement therapy: An update on elderly patients and their outcomes. Clinical Nephrology Journal, 60(5), 352-360.

Jalowiec, A. (1990). Issues in using multiple measures of quality of life. Seminars in Oncology Nursing, 6(4), 271-277.

Janssen Quality-of-life Studies. (2006). Notes on "quality of life ". Retrieved $2^{\text {nd }}$ of June 2006 from http://www.gdrc.org/uem/QOL-define.html 
Jenkinson, C., Stewart-Brown, S., Petersen, S. \& Paice, C. (1999). Assessment of the SF36 version 2 in the United Kingdom. Journal of Epidemiolgy Community Health, $53(1), 46-50$.

Jimenez, R.E., Price, D.A., Pinkus, G.S., Owen, W.F., Lazarus, J.M., Kay, J., et al., (1998). Development of gastrointestinal beta2-microglobulin amyloidosis correlates with time on dialysis. American Journal of Surgical Pathology, 22(6), 729-735.

Jensen, G., Kita, F. Fish, J., Heydt, D., \& Frey, C. (1997). Nutrition risk screening characteristics of rural older persons: Relation to functional limitations and healthcare charges. American Journal of Clinical Nutrition, 66(4), 819-828.

Johnson, T. M. (1996). Cultural considerations. In. B. Spilker (Ed.) Quality of life and pharmacoeconomics in clinical trials. (pp. 511-515). Philadelphia: Lippincott-Raven.

Joyce, C., Hickey, A., McGee, H.M., \& O'Boyle, C.A. (2003). A theory-based method for the evaluation of individual quality of life: The SEIQOL. Quality of life Research, $12(3), 275-280$.

Jungers, P., Massy, Z.A., Nguyen-Khoa, T., Choukroun, T., Robino, C., Fakhouri, R, et al. (2001). Longer duration of predialysis nephrological care is associated with improved long-term survival of dialysis patients. Nephrology Dialysis and Transplantation Journal, 16(12), 2357-2364.

Julius, M., Hawthorne, V.M., Carpenter-Alting, P., Kneisley, J., Wolfe R.A., \& Port, F.K. (1989). Independence in activities of daily living for end stage renal disease patients: Biomedical and demographic correlates. American Journal of Kidney Diseases, 13(1), 61-69.

Kalantar-Zadeh, K., Kopple, J.D., Humphreys, M.H., \& Block G. (2004). Comparing outcome predictability of markers of malnutrition-inflammation complex syndrome in haemodialysis patients. Nephrology Dialysis and Transplantation Journal, 19(6), 1507-1519.

Kalantar-Zadeh. K., McAllister, C.J., Lehn, R.S., Lee, G.H., Nissenson, A.R., \& Kopple, J.D. (2003). Effect of malnutrition-inflammation complex syndrome on EPO hyporesponsiveness in maintenance hemodialysis patients. American Journal of Kidney Disease, 42(4), 761-773.

Kalantar-Zadeh, K., Kopple, J. Block, G. \& Humphreys MH. (2001). Association among SF36 QOL measures and nutrition, hospitalization, and mortality in haemodialysis. Journal of American Society of Nephrology, 12(12), 2797-2806.

Kao, T., Lai, M., Tsai, T Jan, C., \& Chie, W. (2009). Economic, social, and psychological factors associated with health-related quality of life of chronic hemodialysis patients in northern Taiwan: A multicenter study. Journal of Artificial Organs, 33(1), 61-68.

Kapur, R.L. (1992). The family and schizophrenia: Priority areas for intervention research in India. Indian Journal of Psychiatry, 34(1), 3-7. 
K/DOQI clinical practice guidelines for chronic kidney disease. (2002). Evaluation, classification, and stratification. American Journal of Kidney Diseases, 39(1), S1S266.

Khader, S., Hourani, M.M. \& Al-Akour, N. (2011). Normative data and psychometric properties of short form 36 health survey (SF-36, version 1.0) in the population of north Jordan. Eastern Mediterranean Health Journal, 17(5), 368-374.

Khoudri, I., Ali Zeggwagh, A., Abidi, K., Madani, N. \& Abouqal, R. (2006). Measurement properties of the short form 36 and health-related quality of life after intensive care in Morocco. Acta Anaesthesiologica Scandinavica Journal, 51(2), 189-197.

Kimmel, P.L., \& Patel, S.S. (2006). Quality of life in patients with chronic kidney disease: Focus on end-stage renal disease treated with hemodialysis. Seminars in Nephrology, 26(1), 68-79.

Kimmel, P.L., Emont, S.L., Newmann, J.M., Danko, H., \& Moss, A.H.(2003). ESRD patient quality of life: symptoms, spiritual beliefs, psychosocial factors, and ethnicity. American Journal of Kidney Disease, 42(4), 713-721.

Kimmel, P.L. (2002). Depression in patients with chronic renal disease: What we know and what we need to know. Journal of Psychosomatic Research, 53(4), 951-956.

Kimmel, P. (1995). Aspects of quality of life in haemodialysis patients. Journal of American Society Nephrology, 6(5), 1418-1426.

King, C. R., Haberman, M., Berry, D. L., Bush, N., Butler, L., et al. (1997). Quality of life and the cancer experience: The state-of-the-knowledge. Oncology Nursing Forum, 24(1), 27-42.

Kinney, M.R. (1995). Quality of life research: Rigor or rigor mortis. Cardiovascular Nursing, 31(4), 25-28.

Klang, B., Bjorvell, H., Berglund, J., Sundstedt, C., \& Clyne, N. (1998). Predialysis patient education: Effects on functioning and well-being in uraemic patients. Journal of Advanced Nursing, 28(1), 36-44.

Klang, B., \& Clyne, N. (1997). Well-being and functional ability in uraemic patients before and after having started dialysis treatment. Scandinavian Journal of Caring Sciences, 11(3), 159-166.

Ko, B., Khurana, A., Spencer, J., Scott, B., Hahn, M., \& Hammes, M. (2007). Religious beliefs and quality of life in an American inner-city haemodialysis population. Nephrology Dialysis Transplantation Journal, 22(10), 2985-2990.

Korevaar, J.C., Jansen, M.A., Merkus, M.P., Dekker, F.W., Boeschoten, E.W., \& Krediet, R.T. (2002). Quality of life in predialysis end-stage renal disease patients at the 
initiation of dialysis therapy. The NECOSAD Study Group. Peritoneal Dialysis International, 20(1), 69-75.

Kruger, A., Lemke, S., Phometsi, M., Van't Riet, H., Pienaar, A., \& Kotze, G. (2006). Poverty and household food security of black South African farm workers: The legacy of social inequalities. Public Health Nutrition Journal, 9(7), 830-836.

Kutner, N. (2004). Quality of life and daily hemodialysis, Seminars in Dialysis, 17(2), 92-98.

Kutner, N., Bliwise, D., Brogan, D., \& Zhang, R. (2001). Race and restless sleep complaint in older chronic dialysis patients and nondialysis community controls. The Journal of Gerontology, 56(3), 170-176.

Kutner, N.G., Brogan, D., Fielding, B., \& Hall, W.D. (2000). Black/white differences in health outcomes reported by older ESRD patients on chronic hemodialysis. Journal of Ethnicity and Diseases, 10(3), 328-333.

Kutner, N.J. Brogan, D., \& Fielding, B. (1991). Employment status and ability to work among working-age chronic dialysis patients. American Journal of Nephrology, 11(4), 334-340.

Kutner, N.G., \& Devins, G.M. (1998). A comparison of the quality of life reported by elderly whites and elderly blacks on dialysis. Geriatric Nephrology \& Urology Journal, 8(2), 77-83.

Kutner, N.G., Zhang, R., \& Brogan, D. (2005). Race, gender, and incident dialysis patients' reported health status and quality of life. Journal of the American Society of Nephrology, 16(5), 1440-1448.

Laird, L.D., de Marrais, J., \& Barnes, L.L. (2007). Portraying Islam and Muslims in MEDLINE: A content analysis. Social Science \& Medicine, 65(12), 2425-2439.

Laliberte-Rudman, D., Hoffman, L., Scott, E. \& Renwick, R. (2004). Quality of life for individuals with schizophrenia: Validating an assessment that addresses client concerns and occupational issues. The American Occupational Therapy Foundation Journal, 24(1), 13-21.

Lee, J. (2005). An exploratory study on the quality of life of older Chinese people living alone in Hong Kong. Journal of Social Indicators Research, 71(3), 335-361.

Leidy, N. (1994). Functional status and the forward progress of merry-go-rounds: Toward a coherent analytical framework. Nursing Research, 43(4), 196-202.

Leininger, M. M. (1996). Culture care theory, research, and practice. Nursing Science Quarterly, 9(2), 71-78.

Lev, E.L, \& Owen, S.V. (1998). A prospective study of adjustment to hemodialysis. American Nephrology Nurses Association Journal, 25(5), 495-504. 
Levy, A. Brown, J., \& Umw, E. (2004). Handbook of dialysis. Oxford University' Press, Oxford.

Lew, S., \& Piraino, B. (2005). Quality of life and psychological issues in peritoneal dialysis patients. Seminars in Dialysis, 18 (2), 119-123.

Liem, Y.S, Bosch, J.L., Arends, L.R., Heijenbrok-Kal, M.H., \& Hunink, M. (2007). Quality of life assessed with the Medical Outcomes Study Short Form 36-Item Health Survey of patients on renal replacement therapy: A systematic review and meta-analysis. The Journal of International Society of Pharmacoeconomics and Outcome Research, 10(5), 390-397.

Lindqvist, R., Carlsson, M., \& Sjoden, P.O. (2000). Coping strategies and health-related quality of life among spouses of continuous ambulatory peritoneal dialysis, haemodialysis, and transplant patients. Journal of Advanced Nursing, 31(6), 1398408.

Lindstrom, B., \& Eriksson, B. (1993). Quality of life among children in the Nordic countries. Quality of Life Research, 2(1), 23-32.

Lintern, T., Beaumont, J., Kenealy, P., \& Murrell, R. (2001). Quality of life in severely disabled multiple sclerosis patients: Comparison of three quality of life measures using multidimensional scaling. Quality of Life Research, 10(4), 371-378.

Lobo, M. L. (2005). Descriptive research is the bench science of nursing. Western Journal of Nursing Research, 27(1), 5-6.

Locatelli, F., Pisoni, R., Combe, C., Bommer, J., Andreucci, V., Piera, L., et al. (2004). Anaemia in haemodialysis patients of five European countries: Association with morbidity and mortality in the dialysis outcomes and practice patterns study (DOPPS). Nephrology, Dialysis and Transplantation Journal, 19(1), 121-132.

Locatelli, F., Fouque, D., Heimburger, O., Drüeke, T.B., Cannata-Andía, J.B., Hörl, W. et al. (2002). Nutritional status in dialysis patients: A European consensus. Nephrology Dialysis Transplantation Journal, 17(4), 563-572.

Lockridge, R.S., Albert, J., Anderson, H., Barger, T., Coffey, L., Craft, V., et al. (1999). Nightly home hemodialysis: Fifteen months of experience in Lynchburg, Virginia. Home Hemodialysis International, 3(1), 23-28.

Lok, P. (1996). Stressors, coping mechanisms, and quality of life among dialysis patients in Australia. Journal of Advanced Nursing, 23(5), 873-882.

London, G.M., Pannier, B., Guerin, A.P., Blacher, J., Marchais, S.J., Darne, B. et al. (2001). Alterations of left ventricular hypertrophy in and survival of patients receiving hemodialysis: Follow-up of an interventional study. Journal of the American Society of Nephrology, 12(12), 2759-2767. 
Loos, C., Briancon, S., Frimat, L., Hanesse, B., \& Kessler, M. (2003). Effect of endstage renal disease on the quality of life of older patients. Journal of the American Geriatric Society, 51(2), 229-233.

Lopes, A.A., Bragg-Gresham, JL., Goodkin, D.A., Fukuhara, S., Mapes, D.L., Young, E.W., et al. (2007). Factors associated with health-related quality of life among hemodialysis patients in the DOPPS. Quality of Life Research Journal, 16(4), 545557.

Lopes, A., Bragg-Gresham J., Satayathum, S., McCullough, K., Pifer, T., Goodkin, D., et al. (2003). Health-related quality of life and associated outcomes among haemodialysis patients of different ethnicities in the United States: The Dialysis Outcomes and Practice Patterns Study (DOPPS). American Journal of Kidney Diseases, 41(3), 605-615.

Lowe, J., \& Archibald, C. (2009). Cultural diversity: The intention of nursing. Nursing Forum, 44(1), 11-19.

Lowenstein, A. (2007). Solidarity-conflict and ambivalence: Testing two conceptual frameworks and their impact on quality of life for older family members. The Journals of Gerontology, 62(2), 100-108.

Lubeck, DP. (2002). Health-related quality of life measurements and studies in rheumatoid arthritis. American Journal of Managed Care, 8(9), 811-820.

Lukkarinen, H,. \& Hentinen, M. (1998). Assessment of quality of life with the Nottingham health profile among women with coronary artery disease. Heart and Lung: Journal of Acute and Critical Care, 27(3), 189-199.

Lund, I. \& Lundeberg, T. (2008). Is it all about sex? Acupuncture for the treatment of pain from a biological and gender perspective. The British Medical Acupuncture Society, 26(1), 33-45.

Macduff, C. (2000). Respondent-generated quality of life measures: Useful tools for nursing or more fool's gold? Journal of Advanced Nursing, 32(2), 375-382.

Madore, F., Lowrie, E.G., Brugnara, C., Lew, N.L., Lazarus, J.M., Bridges, K. et al. (1997). Anemia in hemodialysis patients: Variables affecting this outcome predictor. Journal of American Society Nephrology, 8(12), 1921-1929.

Mancuso, C.A., Peterson, M.G., \& Charlson, M.E. (2000). Effects of depressive symptoms on health-related quality of life in asthma patients. Journal of General Internal Medicine, 15(5), 301-310.

Manenti, L., Tansinda, P., \& Vaglio, A. (2009). Uraemic pruritus: Clinical characteristics, pathophysiology and treatment. Drugs, 69(3), 251-263.

Mangione, K. (2002). Selection criteria and suggesting used for quality of life measures for elders. Neurology Report, 24(4), 145-151. 
Manns, B., Johnson, J.A., Taub, K., Mortis, G., Ghali, W.A., \& Donaldson, C. (2003). Quality of life in patients treated with hemodialysis or peritoneal dialysis: What are the important determinants? Clinical Nephrology Journal, 60(5), 341-351.

Manns, B.J., Johnson, J.A., Taub, K., Mortis, G., Ghali, W.A. \& Donaldson, C. (2002). Dialysis adequacy and health related quality of life in hemodialysis patients. American Society of Artificial Internal Organs, 48(5), 565-569.

Maor, Y., King, M., Olmer, L., \& Mozes, B. (2001). A comparison of three measures: The time trade-off technique, global health-related quality of life and the SF-36 in dialysis patients. Journal of Clinical Epidemiology, 54(6), 565-570.

Mapes, D.L., Bragg-Gresham, J.L., Bommer, J., Fukuhara, S., McKevitt, P., Wikstrom, B. et al. (2004). Health-related quality of life in the Dialysis Outcomes and Practice Patterns Study (DOPPS). American Journal of Kidney Diseases, 44(3), 54-60.

Maramaldi, P., Berkman, B. \& Barusch, A. (2005). Assessment and the ubiquity of culture: Threats to validity in measures of health-related quality of life. Health \& Social Worker Journal, 30(1), 27-39.

Martinez, S.M, Ainsworth, B.E. \& Elder, J.P. (2008). A review of physical activity measures used among US latinos: Guidelines for developing culturally appropriate measures. Annals of Behavioral Medicine, 36(2), 195-207.

Mazes, B., Shabtai, E., \& Zucker D. (1997). Differences in quality of life among patients receiving dialysis replacement therapy at seven medical centers. Journal of Clinical Epidemiology, 50(9), 1035-1043.

McLaughlin, K., Manns, B., Culleton, B., Donaldson, C., \& Taub K. (2001). An economic evaluation of early versus late referral of patients with progressive renal insufficiency. American Journal of Kidney Diseases, 38(5), 1122-1128.

Merriam-Webster online. (2009). Definition of indicators and determinants. Retrieved 28 November 2009 from: http://www.m-w.com/dictionary

Meeberg, GA. (1993). Quality of life: A concept analysis. Journal of Advanced Nursing, 78(1), 32-38.

Mehrotra, R., Marsh, D., Vonesh, E., Peters, V, \& Nissenson, A. (2005). Patient education and access of ESRD patients to renal replacement therapies beyond in-center hemodialysis. Kidney International, 68(1), 378-390.

Merkus, M., Jager, K., Dekker, F., de Haan, R., Boeschoten, E., \& Krediet, R. (1999). Physical symptoms and quality of life in patient on chronic dialysis: Results of the Netherlands cooperative study on adequacy of dialysis (NECOSAD). Nephrology Dialysis Transplantation Journal, 14(5), 1163-1170. 
Merkus, M.P., Jager, K.J., Dekker, F.W., Boeschoten, E.W., Stevens, P., \& Krediet, R.T. (1997). Quality of life in patients on chronic dialysis: Self-assessment 3 months after the start of treatment. AmericanJournal of Kidney Diseases, 29(4), 584-592.

Mingardi, G., Cornalba, L., Cortinovis, E., Ruggiata, R., Mosconi, P., \& Apolone, G. (1999). Health-related quality of life in dialysis patients. A report from an Italian study using the SF-36 health survey. Nephrology Dialysis Transplantation Journal, 14(6), 15031510.

Mircescu, G., Garneata s., Capusa, C., \& Ursea, N. (2006). Intravenous iron supplementation for the treatment of anaemia in pre-dialyzed chronic renal failure patients. Nephrology Dialysis Transplantation Journal, 2(1), 120-124.

Mitchell, J., \& Goosby, D. (1996). Nutritional management of the long-term care resident receiving haemodialysis. Topics in Clinical Nutrition, 12(1), 57-65.

Mittal, S.K., Ahern, L., Flaster, E., Maesaka, J.K., \& Fishbane, S. (2001). Self-assessed physical and mental function of haemodialysis patients. Nephrology Dialysis Transplantation Journal, 16(7), 1387-1394.

Mok, E., \& Tam, B. (2001). Stressors and coping methods among chronic haemodialysis patients in Hong Kong. Journal of Clinical Nursing, 10(4), 503-511.

Molsted, S., Aadahl, M., Schou, L., \& Eidemak, I. (2004). Self-rated health and employment status in chronic haemodialysis patients. Scandinavian Journal of Urology and Nephrology, 38(2), 174-178.

Molsted, S., Prescott, L., Heaf, J., \& Eidemak, I. (2007). Assessment and clinical aspects of health-related quality of life in dialysis patients and patients with chronic kidney disease. Nephron Clinical Practice Journal, 106(1), 24-33.

Molsted, S., Aadahl, M., Schou, L., \& Eidemak, I. (2004). Self-rated health and employment status in chronic haemodialysis patients. Scandinavian Journal of Urology and Nephrology, 38(2), 174-178.

Montazeri, A., Harirchi, I., Vahdani, M., Khaleghi, F., Jarvandi, S., Ebrahimi, M. et al. (2000). The EORTC breast cancer-specific quality of life questionnaire (EORTC QLQ-BR23): Translation and validation study of the Iranian version. Quality of Life Research Journal, 9(2), 177-184.

Moons, P., Budts, W., \& De Geest, S. (2006). Critique on the conceptualisation of quality of life: A review and evaluation of different conceptual approaches. International Journal of Nursing Studies, 43(7), 891-901.

Moons, P. (2004). Why call it health-related quality of life when you mean perceived health status? European Journal of Cardiovascular Nursing, 3(4), 275-277.

Moons, P., Vanrenterghem, Y., van Hooff, J.P., Squifflet, J.P., Margodt, D., Mullens, et al. (2003). Health-related quality of life and symptom experience in tacrolimus-based 
regimens after renal transplantation: A multicentre study. Transplant International, 16(9), 653-664.

Moreno, F., Lopez, Gomez, J.M., Sanz-Guajardo, D., Jofre, R., \& Valderrabano, F. (1996). Quality of life in dialysis patients: A Spanish multicenter study. Spanish Cooperative Renal Patients Quality of Life Study Group. Nephrology Dialysis Transplantation Journal, 11(2), S125-129.

Morgan, M. (2009). Considering the patient-partner relationship in cancer care: Coping strategies for couples. Clinical Journal of Oncology Nursing, 13(1)65-72.

Morsch, C.M., Gonçalves, L.F., \& Barros, E. (2006). Health-related quality of life among haemodialysis patients--relationship with clinical indicators, morbidity and mortality. Journal of Clinical Nursing, 15(4), 498-504.

Morton, A.R., Meers, C., Singer, M.A., Toffelmire, .EB., Hopman, W., McComb, J. et al. (1996). Quantity of dialysis: Quality of life - what is the relationship? American Society of Artificial Internal Organs, 42(5), 713-717.

Mozes, B, Shabtai, E. \& Zucker, D. (1997). Differences in quality of life among patients receiving dialysis replacement therapy at seven medical centers. Journal of Clinical Epidemiology, 50(9), 1035-1043.

Muldoon, M., Barger, S., Flory, J., \& Manuck, S. (1998). What are quality of life measurements measuring? British Medical Journal, 316(7130), 542-545.

Mustard, C.A., Kuafert, P., \& Kozyrskyj, A. (1998). Sex differences in the use of health care services. New England Journal of Medicine, 338(23), 1678-1683.

Nanda, U. \& Andresen, E..M. (1998). Health-related quality of life. A guide for the health professional. Evaluation and the Health Professions, 21(2), 179-215.

Narayan, MC. (2010). Culture's effects on pain assessment and management. The American Journal of Nursing, 110(4), 38-47.

National Kidney Foundation. (2006). KDOQI clinical practice guidelines and clinical practice recommendations for anemia in chronic kidney disease Update 2006. Retrieved $28^{\text {th }}$ November $28^{\text {th }}$ from : http://www.kidney.org/professionals/kdoqi/guidelines_anemia/index.htm

NKF-K/DOQI (2006). Clinical practice guidelines. Retrieved $20^{\text {th }}$ July 2006 from http://www.kidney.org/professionals/kdoqi/guidelinesupdates/doqiupan_i.html\#2

Neill, J. (2008). Analysis of professional literature class 8: Qualitative research 3 (content analysis \& literature review writing skills). Article retrieved $25^{\text {th }}$ September 2008 from http://wilderdom.com/OEcourses/PROFLIT/Class8Qualitative3.htm 
Neri, L., Gallieni, M., Cozzolino, M., Rocca-Rey, L., Brambilla, G., Colombi, A. et al. (2005). Work ability and health status in dialysis patients. International Journal of Artificial Organs, 28(6), 554-556.

Neto, J.F., Ferraz, M.B., Cendoroglo, M., Draibe, S., Yu, L. \& Sesso, R. (2000). Quality of life at the initiation of maintenance dialysis treatment: A comparison between the SF36 and KDQ questionnaires. Quality of Life Research Journal, 9(1), 101-107.

Nettina, S.M. (2006). Lippincott manual of nursing practice: Manual of nursing practice. $\left(8^{\text {th }}\right.$ ed). Lippincott Williams \& Wilkins. Philadelphia. USA.

Newby, N. (1996). Chronic illness and the family life-cycle. Journal of Advanced Nursing, 23(4), 786-791.

Neuman, W. (2006). Social Research methods qualitative and quantitive approaches. (6 $6^{\text {th }}$ ed.). Boston. Pearson.

Nicole, M., \& Harada, A. (1999). Measuring the effect of medication use on health related quality of life in patients with rheumatoid arthritis. Pharmacoeconomics, 16(5), 433448.

NKF-KDOQI clinical practice guidelines. (2006). American Journal of Kidney Diseases, 48(1), S2-S90.

O’Boyle, C.A., McGee, H., Hickey, A., O’Malley, K. \& Joyce, C.R. (1992). Individual quality of life in patients undergoing hip replacement. Lancet, 339(8801), 1088-1091.

O’Brien, B.J., Spath, M., Blackhouse, G., Severens, J.L, \& Brazier, J.E. (2003). A view from the Bridge: Agreement between the SF-6D utility algorithm and the Health utilities Index. Health Economics, 12(11), 975-982.

Oman, K.S., Krugman, M.E. \& Fink, R.M. (2003). Nursing research secrets. Philadelphia, Pennsylvania. Hanly \& Belfus, INC.

Oppenheim, A.N. (2001). Questionnaire design, interviewing and attitude measurement. Retrieved February $25^{\text {th }}, 2010$ from: http://books.google.co.nz/books?hl=en\&lr=\&id=6V4GnZS7TO4C\&oi=fnd\&pg=PA1\&d $q=a d v a n t a g e s+o f+D e s c r i p t i v e+s u r v e y+d e s i g n+p d f \& o t s=s z 177 q U E e l \& s i g=t k v L-$ kBYjv2o7AVuSwHL1aYI1DU\#v=onepage \&q=\&f=false

Orley, J. (1994). The World Health Organization (WHO) quality of life project. In M. R. Trimble, \& W. E. Dodson (Eds.), Epilepsy \& quality of life (pp. 99-133). New York: Raven Press.

Ontario Social Development Council. (2006). Notes on "quality of life ". Retrieved $2^{\text {nd }}$ June 2006 from http://www.gdrc.org/uem/QOL-define.html 
Owen, W.F., Lew, N.L., Liu, Y., Lowrie, E.C., \& Lazarus, J.M. (1993).The urea reduction ratio and serum albumin concentrations as predictors of mortality in patients undergoing hemodialysis. New England Journal of Medicine, 329(14), 1001-1006.

Pai, M.F., Hsu, S.P., Yang, S.Y., Ho, T.I., Lai, C.F., Peng, Y.S. (2007). Sleep disturbance in chronic hemodialysis patients: The impact of depression and anemia. Renal Failure, 29(6), 673-677.

Pakpour, A., Saffari, M., Yekaninejad, S., Panahi, D., Harrison, A., \& Molsted, S. (2010). Health-Related quality of life in a sample of Iranian patients on hemodialysis. Iranian Journal of Kidney Diseases, 4(1), 50-59.

Palmer, BF. (2003). Sexual dysfunction in men and women with chronic kidney disease and end-stage kidney disease. Advancement in Renal Replacement Therapy Journal, 10(1), 48-60.

Pallant, J. (2007). SPSS survival manual. (3 $3^{\text {rd }}$ ed.). Mc Graw Hill. London, UK.

Palmer, B.F. (2003). Sexual dysfunction in men and women with chronic kidney disease and end-stage kidney disease. Advancement in Renal Replacement Therapy Journal, 10(1), 48-60.

Parker, K.P., Bliwise, D.L., Bailey, J.L., \& Rye, D.B. (2005). Polysomnographic measures of nocturnal sleep in patients on chronic, intermittent daytime haemodialysis vs those with chronic kidney disease. Nephrology Dialysis Transplantation Journal, 20(7), $1422-1428$.

Peat, J., \& Barton, B. (2006). Medical statistics: A guide to data analysis and critical appraisal. ( $2^{\text {nd }}$ ed.). Blackwell Publishing. New Delhi, India.

Patel, S., Shah, V., Peterson, R., \& Kimmel, P. (2002). Psychosocial variables, quality of life, and religious beliefs in ESRD patients treated with haemodialysis. American Journal of Kidney Diseases, 40(5), 1013-1022.

Pejman, P. (2007). Economy-UAE: Indians, Pakistanis fuel fresh real estate boom. Retrieved $23^{\text {rd }}$ October 2009 from: http://ipsnews.net/news.asp?idnews=37177

Peiris, D., Brown, A., \& Cass, A. (2008). Addressing inequities in access to quality health care for indigenous people. Canadian Medical Association Journal, 179(10), 985986.

Peplau, H.E. (1994). QOL: An interpersonal perspective. Nursing Science Quarterly, 7(1), 10-15.

Perez, L. Huang, J., Jansky, L., Nowinski, C., Victorson, D, Peterman, A., et al. (2007). Using focus groups to inform the Neuro-QOL measurement tool: Exploring patientcentered, health-related quality of life concepts across neurological conditions. Journal of Neuroscience Nursing, 39(6), 342-53. 
Pierce, C.A., Block, C.A., \& Aguinis, H. (2004). Cautionary note on reporting Eta-squared values from multifactor Anova designs. Educational and Psychological Measurement Journal, 64(6). Retrieved $1^{\text {st }}$ August 2010 from : http://en.wikiversity.org/wiki/Etasquared

Pifer, T., Bragg-Gresham, J., Dykstra, D., Shapiro, et al. (2003). Quality of life and patient satisfaction: ESRD managed care demonstration. Healthcare Financing Review, 24(4), 45-58.

Pincus, T., \& Wolfe, F. (2000). An infrastructure of patients questionnaires at each rheumatology visit: Improving efficacy and documenting care. The Journal of Rheumatology, 27(12), 2727-2729.

Polaschek, N. (2003). Living on dialysis: Concerns of clients in a renal setting. Journal of Advanced Nursing, 41(1), 44-52.

Polit, D. F. \& Beck, C. T. (2004). Nursing research: Principles and methods. (7 ${ }^{\text {th }}$ ed.). Philadelphia: Lippincott, Williams \& Wilkins.

Pope, C., Ziebland, S., \& Mays, N. (2002). Qualitative research in health care: Analysing qualitative data. British Medical Journal, 320 (7227), 114-116.

Power, A., Duncan, N., \& Goodlad, C. (2009). Management of the dialysis patient for the hospital physician. Postgraduate Medical Journal, 85(1005), 376-381.

Price, V.E, Klaassen, R.J, Bolton-Maggs, P.H, Grainger, J.D, Curtis, C, Wakefield, C, et al., (2009). Measuring disease-specific quality of life in rare populations: A practical approach to cross-cultural translation. Health Quality of Life Outcomes, 7(92), doi:10.1186/1477-7525-7-9. Retrieved $25^{\text {th }}$ November 2011 from: http://www.hqlo.com/content/7/1/92

Prince, P. \&, Gerber, G. (2001). Measuring subjective quality of life in people with serious mental illness using the SEIQOL-DW. Quality Of Life Research, 10(2), 117-122.

Purnell, D. L., \& Paulanka, J. B. (2005). Guide to culturally competent health care Philadelphia: F.A. Davis Company.

QOL Research Unit, University of Toronto. (2006). Notes on "quality of life ". Retrieved $2^{\text {nd }}$ June 2006 from http://www.gdrc.org/uem/QOL-define.html

Quality-of-Life Research Center, Denmark. (2006). Notes on "quality of life ". Retrieved $2^{\text {nd }}$ June 2006 from: http://www.gdrc.org/uem/QOL-define.html

Quality Metric tools. (2011). The Translation Process of SF-36 tool. Retrieved $24^{\text {th }}$ August 2011 from http://www.qualitymetric.com/WhatWeDo/LanguageTranslations/tabid/213/Default.as $\mathrm{px}$ 
Quality Metric tools. (2009). The SF-12 $2^{\oplus}$ : An Even Shorter Health Survey. Retrieved $28^{\text {th }}$ May 2009 from http://www.sf-36.org/tools/sf12.shtml

Quality Metric Scoring Program. (2007). Users Manual for the SF-36v2 ${ }^{\mathrm{TM}}$ Health Survey. Article retrieved on $12^{\text {th }}$ September 2008 http://www.qualitymetric.com/products/ProductDetails.aspx?productID=563\&category id $=1$

Quality Metric. (2009). The SF-12 $2^{\circledR}$ : An Even Shorter Health Survey. Retrieved $2^{\text {nd }}$ June 2006 from http://www.sf-36.org/tools/sf12.shtml

Qunibi, W. (2004). Consequences of hyperphosphatemia in patients with end-stage renal disease (ESRD). Kidney International, 66(90), 8-12.

Rabetoy, C. (2007). Who should be diagnosing, prescribing, and managing depression in patients with ESRD? Nephrology Nursing Journal, 34(5), 554-557.

Rajkumar, S. \& Kumar, S. (1996). Strengths and limitations in using psychiatric measures across cultures. Indian Journal of Psychiatry, (4) 240-247.

Rambod, M., Bross, R., Zitterkoph, J., Benner, D., Pithia, J., Colman, S., et al. (2009). Association of Malnutrition-Inflammation Score with quality of life and mortality in hemodialysis patients: A 5-year prospective cohort study. American Journal of Kidney Disease, 53(2), 298-309.

Ramirez, M., Ford, M., Stewart, A. \& Teresi. J. (2005). Measurement issues in health disparities research. Health Services Research Journal, 40(5), 1640-1657.

Rassool, G. H. (2000). The crescent and Islam: Healing, nursing and the spiritual dimension. Some considerations towards an understanding of the Islamic perspectives on caring. Journal of Advanced Nursing, 32(6), 1476-1484.

Rebollo, P., Alvarez-Ude, F., Valdes, C., Estebanez, C. \& FAMIDIAL Study Group. (2005). Different evaluations of the health related quality of life in dialysis patients. Journal of Nephrology, 17(6), 833-840.

Resnicow, K., Dilorio, C., Soet, J., Ernst, D., Borrelli, B. \& Hecht, J. (2002). Motivational interviewing in health promotion: It sounds like something is changing. Health Psychology, 21(5), 444-451.

Rittman, M., Northsea, C., Hausauer, N., Green, C., \& Swanson, L. (1993). Living with renal failure. American Nephrology Nursing Association Journal, 20(3), 327-331.

Roshan Cultural Heritage Institute definition of culture (2011). Retrieved $7^{\text {th }}$ December 2011 from http://www.roshan-institute.org/474552

Roth, T. (2007). Insomnia: Definition, prevalence, etiology, and consequences. Journal of Clinical Sleep Medcine, 3(5), 7-10. 
Roth, T. \& Roehrs, T. (2003). Insomnia: Epidemiology, characteristics, and consequences. Clinical Cornerstone, 5(3), 5-15.

Rubin, H.R., Fink, N.E., Plantinga, L.C., Sadler, J.H., Kliger, A.S., \& Powe, N.R. (2004). Patient ratings of dialysis care with peritoneal dialysis vs hemodialysis. Journal of American Medical Associations, 291(6), 697-703.

Ruo, B., Rumsfeld, J.S., Hletky, M.A., Liu, H., Browner, W.S., \& Whooley, M.A., (2003). Depressive symptoms and health related quality of life: the heart and soul study. JAMA 290(2), 215-221.

Sabbah, I.,Drouby, N., Sabbah, S., Retel-Rude, N. \&, Mercier, M. (2003). Quality of life in rural and urban populations in Lebanon using SF-36 health survey. Health Quality of Life Outcomes Journal. 1(30), doi:10.1186/1477-7525-1-30. Retrieved on September $1^{\text {st }} 2011$ from http://www.hqlo.com/content/pdf/1477-7525-1-30.pdf

Sabbatini, M, Minale, B, Crispo, A, Pisani, A, Ragosta, A, Esposito, R., et al., (2002). Insomnia in maintenance haemodialysis patients. Nephrology Diaysis Transplanation Journal, 17(5), 852-856.

Safran, D.G., Rogers, W.H., Tarlov, A.R., McHorney, C.A., \& Ware, J.E. (1997). Gender differences in medical treatment: The case of physician-prescribed activity restrictions. Social Science \& Medicine Journal, 45(5), 711-722.

Salleh, K.M. (1994) The islamic perspectives of caring. Resolutions and recommendations. Islamic Caring Practices and Management in Nursing. Proceedings of the First International Nursing Conference: Education for Caring. Pengiran Anak Puteri Rashidah Sa'adatul Bolkiah, College of Nursing, Brunei Darussalam.

Sanaka, T. (2003). Nutritional effect of dialysis therapy. Artificial Organs Journal, 27(3), 224-226.

Santos, P.R. \& Kerr, L.R. (2008). Clinical and laboratory variables associated with quality of life in Brazilian haemodialysis patients: A single-centre study. Revista Medica Chile, 136(10), 1264-1271.

Sarason, I. G., Sarason, B. R., \& Pierce, G. R. (1990). Social support: The search for theory. Journal of Social and Clinical Psychology, 9(1), 133-147.

Sartorius,N. \& Kuyken, W. (1994). Trans-lation of health status instruments. In J. Orley \& W. Kuyken (eds), Quality of life assessments. International perspectives. (pp. 3 17)Proceedings of WHO and Foundation IPSEN Paris meeting, Springer-Verlag Publication.

Sayin, A., Mutluay, R., \& Sindel, S. (2007). Quality of life in hemodialysis, peritoneal dialysis, and transplantation patients. Transplant Proceedings Journal, 39(10), 30473053. 
Schalock, R. (2004). The concept of quality of life: What we know and do not know. Journal of Intellectual Disability Research, 48 (3), 203-216.

Schatell, D., \& Witten, B. (2004). Anaemia: Dialysis patients' experiences. Nephrology News \& Issues. 18(12), 49-54.

Schmidt, R.J., Domico, J.R., Sorkin, M.I., \& Hobbs, G. (1998). Early referral and its impact on emergent first dialyses, health care costs, and outcome. American Journal of Kidney Diseases, 32(2), 278-283.

Schweikert, B., Hunger, M., Meisinger, C., König, H. Gapp. O., \& Holle, R. (2009). Quality of life several years after myocardial infarction: Comparing the MONICA/KORA registry to the general population. European Heart Journal, 30(4), 436-444.

Scott, N., Fayers, P., Aaronson, N., Bottomley, A., de Graeff, A., Groenvold, M., et al. (2008). The relationship between overall quality of life and its subdimensions was influenced by culture: Analysis of an international database. Journal of Clinical Epidemiology, 61(8), 788-795.

Sen B. \& Man, J. (1986) Psychiatric research instruments in the transcultural setting: Experiences in India and Brazil. Social Science and Medicine Journal, 23(3), 277281.

Shidler, N.R, Peterson, R.A, \& Kimmel, P.L. (1998). Quality of life and psychosocial relationships in patients with chronic renal insufficiency. American Journal of Kidney Diseases, 32(4), 557-566.

Shuraida, S. S. (2001). Islamic activism. American Muslim, 2(4), 8-10.

Sijtsma, K., Emons, W., Bouwmeester, S., Nyklíček, I. \& Roord, L. (2008), Nonparametric IRT analysis of Quality-of-Life Scales and its application to the World Health Organization Quality-of-Life Scale (WHOQOL-Bref). Quality of Life Research Journal, 17(2), 275-290.

Smith, J. (1999). Islam in America. New York: Columbia University Press

Simko, L. (1999). Adults with congenital heart disease: Utilizing quality of life and Husted's nursing theory as a conceptual framework. Critical Care Nursing Quarterly, 22 (3), 111.

Smith, K.W., Avis, N.E., \& Assmann, S.F. (1999). Distinguishing between quality of life and health status in quality of life research: A meta-analysis. Quality of Life Research, $8(5), 447-459$.

Sørensen, V.R., Mathiesen, E.R., Watt, T., Bjorner, J.B., Andersen, M.V., \& FeldtRasmussen, B. (2007). Diabetic patients treated with dialysis: Complications and quality of life. Diabetologia Journal, 50(11), 2254-2262. 
Speigel, D.M. (2006). Anemia management in chronic kidney disease: what have we learned after 17 years? Seminars in Dialysis, 19(4), 269-272.

Spiegel, B.M., Melmed, G., Robbins, S., \& Esrailian, E. (2008). Biomarkers and healthrelated quality of life in end-stage renal disease: A systematic review. Clinical Journal of American Society of Nephrology, 3(6), 1759-1768.

Spinale, J., Cohen, S.D., Khetpal, P., Peterson, R.A., Clougherty, B., Puchalski, C.M., Pate,I S.S. \& Kimmel, P.L. (2008). Spirituality, social support, and survival in hemodialysis patients. Clinical Journal American Society of Nephrology, 3(6), 16201627.

Sprangers, M.A., Groenvold, M., Arraras, J.I., Franklin, J., te Velde, A., Muller, M. et al. (1996). The European organization for research and treatment of cancer specific quality-of-life questionnaire module: First results from a three country field study. Journal of Clinical Oncology, 14(10), 2756-2768.

Springhouse. (2005). Professional Guide to Diseases $\left(8^{\text {th }}\right.$ ed). Lippincott Williams \& Wilkins. Philadelphia. USA.

Stapleton, S. (2000). Powerlessness in persons with end-stage renal disease. In Miller F (ed). Coping with chronic illness: Overcoming powerlessness (3rd ed). FA Davis Co, Philadelphia.

Steele, T., Baltimore, D., Flnkselstein, S., Juergensen, P., Kliger, A., \& Flnkselstein, F. (1996). Quality of Life in peritoneal dialysis patients. Journal of Nervous and Mental Disease, 184(6), 368-374.

Steele, T.E., Wuerth, D., Finkelstein, S., Juergensen, D., Juergensen, P., Kliger, A.S. et al., (1996). Sexual experience of the chronic peritoneal dialysis patient. Journal of the American Society of Nephrology, 7(8), 1165-1168.

Sterky, E., \& Stegmayr, B.G. (2005). Elderly patients on haemodialysis have $50 \%$ less functional capacity than gender- and age-matched healthy subjects. Scandinavian Journal of Urology \& Nephrology, 39(5), 423-430.

Stewart, A. \& Napoles-Springer, A. (2000). HRQL assessment in diverse population groups in the US. Medical Care Journal, 38 (9), 102-124.

Suet-Ching W.L. (2001). The quality of life Hong Kong dialysis patients. Journal of Advanced Nursing, 35(2), 218-227.

Stewart, A. (1992). Conceptual and methodologic issues in defining quality of life: State of the art. Progress in Cardiovascular Nursing, 7(1), 3-11.

Stuifbergen, A. \& Barbara, B. (2005). Secondary conditions and life satisfaction among polio survivors/commentary. Rehabilitation Nursing, 30(5), 173-177. 
Takaki, J., Nishi, T., Shimoyama, H., Inada, T., Matsuyama, N., Kumano, H. et al. (2005). Possible interactive effects of demographic factors and stress coping mechanisms on depression and anxiety in maintenance hemodialysis patients. Journal of Psychosomatic Research, 58(3), 217-223.

Tempelaar, R., De Haes, J.C., De Ruiter, J.H., Bakker, D., Van Den Heuvel, W.J., \& Van Nieuwenhuijzen, M.G. (1989). The social experiences of cancer patients under treatment: A comparative study. Social Science in Medicine, 29(5), 635-642.

Tentori.F. (2008). Focus on: Physical exercise in hemodialysis patients. Journal of Nephrology, 21(6), 808-812.

Testa, M., \& Simonson, D. (1996). Assessment of quality of life outcomes. New England Journal of Medicine, 334(13), 835-840.

The National Institute for Urban School Improvement. (2011). Understanding culture. Retrieved $18^{\text {th }}$ November 2011 from http://urbanschools.org/pdf/understanding.culture.LETTER.pdf

Thong, M.S., Kaptein, A.A., Krediet,R.T., Boeschoten, E.W., \& Dekker, F.W. (2007). Social support predicts survival in dialysis patients. Nephrology Dialysis and Transplantation, 21(12), 845-850.

Tsay, S.L., \& Healstead, M. (2002). Self-care self-efficacy, depression, and quality of life among patients receiving hemodialysis in Taiwan. International Journal of Nursing Studies, 39(8), 245-251.

Tsay, S.L., Lee, Y.C., \& Lee, Y.C. (2005). Effects of an adaptation training programme for patients with end-stage renal disease. Journal of Advanced Nursing, 50(1), 39-46.

Tseng, H.M., Lu, J.F., \& Gandek, B. (2003). Cultural issues in using the SF-36 Health Survey in Asia: Results from Taiwan. Health and Quality of Life Outcomes, 1, 72. doi:10.1186/1477-7525-1-72. Retrieved February $1^{\text {st }} 2012$ from http://www.hqlo.com/content/pdf/1477-7525-1-72.pdf

Tsuji-Hayashi, Y., Fitts, S.S., Takai, I., Nakai, S., Shinzato, T., Miwa, M., et al. (2001). Health-related quality of life among dialysis patients in Seattle and Aichi. American Journal of Kidney Disease, 37(5), 987-996.

Thomas, N.T. (2002). Renal Nursing. 2nd ed. Baiilière Tindall. London

Tooth, L., Russell, A., Lucke, J., Byrne, G., Lee, C., Wilson, A. \& Dobson, A. (2008). Impact of cognitive and physical impairment on carer burden and quality of life. Quality of Life Research, 17(2), 267-273.

Twardowski, Z.J. (2004). Short, thrice-weekly hemodialysis is inadequate regardless of small molecule clearance. International Journal of Artificial Organs, 27(6), 452-466. 
UAE Census. (2005). Preliminary results of population, housing and establishments census 2005 United Arab Emirates. Retrieved 20 ${ }^{\text {th }}$ July 2008 from www.tedad.ae/english/results.pdf

Ulrich, B. (2009). Providing culturally competent nursing care. Nephrology Nursing Journal, 36(4)367-368.

Unruh, M.L., Weisbord, S.D., \& Kimmel, P.L. (2005). Health-related quality of life in nephrology research and clinical practice. Seminars in Dialysis, 18(2), 82-90.

Unruh, M.L., \& Hess, R. (2007). Assessment of health-related quality of life among patients with chronic kidney disease. Advances in Chronic Kidney Disease Journal, 14(4), 345-352.

Unruh, M., Benz, R., Greene, T., Yan, G., Beddhu, S., DeVita, M., et al. (2004). Effects of hemodialysis dose and membrane flux on health-related quality of life in the HEMO Study. Kidney International, 66(1), 355-366.

US Renal Data System. USRDS (2001). Annual Data Report: The National Institutes of Health, National Institute of Diabetes and Digestive and Kidney Diseases, Bethesda, MD.

Valderrabano, F., Jofre, R., \& Lopez-Gomez, J. (2001). Quality of life in end-stage renal disease patients. American Journal of Kidney Disease, 38(3), 443-464.

Valderrabano, F. (1996). Erythropoietin in chronic renal failure. Kidney International, 50(4), 1373-1391.

Vasilieva, I. (2006). Quality of life in chronic hemodialysis patients in Russia. Hemodialysis International Journal, 10(3), 274-278.

Van Manen, J.G., Korevaar, J.C., Dekker, F.W., Boeschoten, E.W., Krediet, R.T; NECOSAD Study Group. Netherlands cooperative study on adequacy of dialysis. (2001). Changes in employment status in end-stage renal disease patients during their first year of dialysis. Peritoneal Dialysis International, 21(6), 595-601.

Van Wyk, F.C., Vaz, F., Harries, M., \& Weighill, J. (2008). Is there a difference in quality of life, comparing summer and winter, in patients who have undergone total laryngectomy? The Journal of Laryngology \& Otology, 122(9), 967-971.

Van Widenfelt, B.M., Treffers, P.D., De Beurs, E., Siebelink, B.M. \& Koudijs, E. (2005). Translation and cross-cultural adaptation of assessment instruments used in psychological research with children and families. Clinical Child and Family Psychology Review, 8(2), 135-142.

Veenhoven, R. (2000). The four qualities of life: Ordering concepts and measures of the good life. Journal of Happiness Studies, 1(3), 1-39. 
Vega, W. A. (1992). Theoretical and pragmatic implications of cultural diversity for community research. American Journal of Community Psychology, 20(3), 375-391.

von Elm, E., Altman, DG., Egger, M., Pocock, S.J., Gøtzsche, P.C., \& Vandenbroucke, J.P.; STROBE Initiative. (2007). The strengthening the reporting of observational studies in epidemiology (STROBE) statement: Guidelines for reporting observational studies. Lancet, 370(9596), 1453-1457.

Waldron, D., O'Boyle, C.A., Kearney, M., Moriarty, M., \& Carney, D. (1999). Quality-of-life measurement in advanced cancer: Assessing the individual. Journal of Clinical Oncology, 17(11), 3603-3611.

Wagner, J., Abbott, G., \& Lett, S. (2004). Age related differences in individual QOL domains in youth with type 1 diabetes. Health and QOL Outcomes, 2(54), 1-9.

Ware, J. (1993). SF-36 health survey manual \& interpretation guide. Boston, Mass: The Medical Outcome Trust.

Ware, J. \& Sherbourne, C. (1992). The MOS 36-Item Short-Form Health Survey (SF-36): Conceptual framework and item selection. Medical Care, 30(6), 473-483.

Warnecke, RB., Ferrans, CE., Johnson, TP., Chapa-Resendez, G., O'Rourke, DP., Chávez, N., et al. (1996). Measuring quality of life in culturally diverse populations. Journal of the National Cancer Institute Monographs, (20), 29-38.

Weinberg, G., Hoffmann, K., Pohle, C., Hampel, D. \& Schindler, R. (2007). Back pain in a haemodialysis patient with severe atherosclerosis. Nephrology, Dialysis, Transplantation, 22(11), 3349-3352.

Weisbord, SD., Fried, L.F., Arnold, R.M., Fine, M.J., Levenson, D.J., Peterson, R.A. (2005). Prevalence, severity, and importance of physical and emotional symptoms in chronic hemodialysis patients. Journal of American Society of Nephrology, 16(8), 2487-2494.

Weisbord, S.D, Bossola, M, Fried, L.F, Giungi, S, Tazza, L, Palevsky, P.M, et al. (2008). Cultural comparison of symptoms in patients on maintenance hemodialysis. Hemodialysis International Journal, 12(4), 434-440.

Welch, J., \& Austin, J. (2001). Stressors, coping and depression in haemodialysis patients. Journal of Advanced Nursing, 33(2), 200-207.

Wettergren, L., Bjorkholm M., \& Langius-Eklof, A. (2005). Validation of an extended version of the SEIQOL-DW in a cohort of Hodgkin lymphoma' survivors. Quality of Life Research, 14(10), 2329-2333.

WHO Quality of life-BREF (2006). WHO QOL-BREF. Retrieved $2^{\text {nd }}$ June 2006 from http://www.who.int/substance_abuse/research_tools/whoQOLbref/en/ 
Wilkinson, J.C. (2009). From Liwa to AbuDhabi. Journal of National Center for Documentation and Research, 1(1), 5-11.

WI, S. (2001). The quality of life for Hong Kong dialysis patients. Journal of Advanced Nursing, 35(2), 218-227.

Women of the UAE. (2007). Employment. Retrieved $2^{\text {nd }}$ May 2007 from http://www.datadubai.com/women.htm

Wright, S. (1999). How to be happy at work. Nursing Times, 95(35), 26-28.

Wyss, K., Wagner, A.K., Whiting D., Mtasiwa, D.M \& Tanner, M. et al. (2000). Psychometric evaluation of a Chinese (Taiwanese) version of the SF-36 health survey amongst middle-aged women from a rural community. Quality of Life Research Journal, 9(6), 675-683.

Yabroff, R., Linas, B., \& Schulman, K. (1996). Evaluation of quality of life for diverse patient populations. Breast Cancer Research and Treatment Journal, 40(1), 87-104.

Yarlas, A.S., White, M.K., Yang, M., Saris-Baglama, R.N., Bech, P.G. \& Christensen, T. (2011). Measuring the health status burden in hemodialysis patients using the SF36_ health survey. Quality of Life Research Journal, 20(3), 383-389.

Yildirim, A., Akinci, F., Gozu, H., Sargin, H., Orbay, E. \& Sargi, M. (2007). Translation, cultural adaptation, cross-validation of the Turkish diabetes quality-of-life (DQOL) measure. Quality of Life Research Journal, 16(5), 873-887.

Zarifian, A. (1994). Case study of the anemic patient: Epoetin alfa-focus on sexual function. American Nephrology Nursing Association Journal, 21(6), 368-371.

Zhan, L. (1992). Quality of Life: Conceptual and measurement issues. Journal of Advanced Nursing, 77(7), 795-800.

\section{Bibliography}

Sources of the Holy Qu'ran Text. (2007). Retrieved $2^{\text {nd }}$ May 2007 from http://www.quranexplorer.com/ 